\title{
Finite elements in computational electromagnetism
}

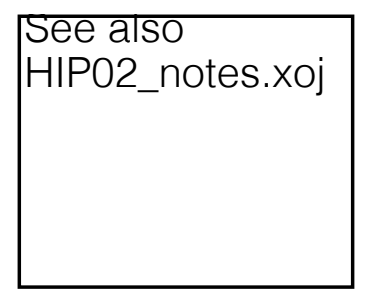

\author{
R. Hiptmair \\ Sonderforschungsbereich 382, \\ Universität Tübingen, \\ D-72076 Tübingen, Germany \\ E-mail: ralf@hiptmair.de
}

This article discusses finite element Galerkin schemes for a number of linear model problems in electromagnetism. The finite element schemes are introduced as discrete differential forms, matching the coordinate-independent statement of Maxwell's equations in the calculus of differential forms. The asymptotic convergence of discrete solutions is investigated theoretically. As discrete differential forms represent a genuine generalization of conventional Lagrangian finite elements, the analysis is based upon a judicious adaptation of established techniques in the theory of finite elements. Risks and difficulties haunting finite element schemes that do not fit the framework of discrete differential forms are highlighted.

\section{CONTENTS}

1 Introduction 238

2 Maxwell's equations 240

3 Discrete differential forms 255

4 Maxwell eigenvalue problem 291

5 Maxwell source problem 306

6 Regularized formulations 318

7 Conclusion and further issues $\quad 327$

References $\quad 328$

Appendix: Symbols and notation 337 


\section{Introduction}

Most modern technology is inconceivable without harnessing electromagnetic phenomena. Hence the design and analysis of schemes for the approximate solution of electromagnetic field problems can claim a rightful place as a core discipline of numerical mathematics and scientific computing. However, for a long time it received far less attention among numerical analysts than, for instance, computational fluid dynamics and solid mechanics.

One reason might be that electromagnetism is described by a generically linear theory, in the sense that linear equations arise from basic physical principles. This is in stark contrast to continuum mechanics, where linear models only emerge through linearization of inherently nonlinear governing principles. Being linear, the fundamental laws of electromagnetism might have struck many mathematicians as 'dull'. This view might also have been fostered by the misconception that electromagnetism basically boils down to plain second-order elliptic equations, which have been amply studied and are well understood.

It is one objective of this survey article to refute the idea that one can cope with electromagnetics once one knows how to solve Laplace equations numerically. I aim to convey the richness in subtle mathematical features displayed by apparently 'simple' problems in computational electromagnetism. The problems I have in mind arise from the spatial discretization of electromagnetic fields by means of finite elements. Yet I will not settle for merely specifying and describing the finite element spaces. To gain insight, a comprehensive view is mandatory, encompassing the structural aspects of the physical model, a thorough knowledge of function spaces as well as familiarity with classical finite element techniques. All these issues will be addressed in the paper, and an attempt is made to convince the reader that understanding all of them is necessary for successfully tackling electromagnetic field problems.

Many readers might object to my regular delving into technical details. In my opinion, major breakthroughs in computational electromagnetism have often been brought about by successfully addressing technical issues. This neatly fits my desire to embrace a formal 'rigorous' treatment. Therefore, space permitting, and in order to make the article self-contained, I will not skip proofs. Yet, sometimes I will put forth 'views' - even at the risk of sounding fuzzy and arcane - in a possibly doomed attempt to inspire 'intuitive understanding'.

Plenty of references to original papers and related work will be given. Of course, they can never be exhaustive and will reflect my personal biases and history. In particular, scores of engineering publications that address issues also covered in this article could be cited, but will not be mentioned. My 
emphasis on theory is reflected by the almost complete absence of numerical results. They can be found in abundance in research papers.

I was pleased to witness a surge in research activities into mathematical aspects of computational electromagnetism in recent years. Now the field is rapidly evolving, which means that this article can hardly be more than a snapshot of the knowledge as of 2001. Many of the results covered are likely to experience significant improvement and extension in years to come. It also means that there is much left to be done. In a sense, I will not balk at stating incomplete results and even conjectures. Maybe this will trigger some fresh research.

Even with a focus on finite element schemes, all that can be covered in a survey article are model problems. Admittedly, they fall way short of matching the complexity of typical engineering applications. For instance, in light of the linear nature of electromagnetism, I will completely restrict my attention to linear problems, that is, only simple 'linear' materials will be considered. In this setting it is possible to skirt any issues of temporal discretization by switching to the frequency domain: all quantities are supposed to show a sinusoidal dependence on time with a fixed angular frequency $\omega>0$. Thus, thanks to linearity, temporal derivation $\partial_{t}$ can be replaced by the multiplication operator $i \omega$. This converts all equations into relationships between complex amplitudes depending on space only. If $\mathbf{u}=\mathbf{u}(\mathbf{x}), \mathbf{x} \in \mathbb{R}^{3}$ standing for the independent space variable, is such a complex amplitude, the related physical quantity $\mathrm{U}$ can be recovered through

$$
\mathrm{U}(\mathbf{x}, t)=\operatorname{Re}(\mathbf{u}(\mathbf{x}) \cdot \exp (i \omega t)) .
$$

The classical notion of finite elements is tied to bounded computational domains. Yet many central problems in computational electromagnetism are posed on unbounded domains. The most prominent example is the scattering of electromagnetic waves. Not all of these problems will be fully treated in this article. Still, when combined with other techniques, for instance boundary element methods, finite elements can play an important role even in these cases. Thus the results reported in this article remain of interest.

Instead of an outline, I am only listing a few points of view that I embrace. They can offer guidance when negotiating through this article.

- In order to discretize the fundamental laws of electromagnetism properly, it is important to appreciate their link with differential geometry and algebraic topology (cohomology theory).

- There is a close relationship between second-order elliptic equations and the governing equations of electromagnetism, but the lack of strong ellipticity introduces subtle new challenges.

- Suitable finite elements for electromagnetic fields should be introduced and understood as discrete differential forms. 
- Discrete differential forms are a generalization of $H^{1}(\Omega)$-conforming Lagrangian finite elements. Their analysis can often use and adapt the tools developed for the latter.

- Finite elements that lack an interpretation as discrete differential forms have to be used with great care.

\section{Maxwell's equations}

The fundamental governing equations of electromagnetism are Maxwell's equations. Mathematicians usually encounter them in the form of the two first-order partial differential equations

$$
\begin{array}{ll}
\text { Faraday's law: } & \operatorname{curl} \mathbf{e}=-i \omega \mathbf{b}, \\
\text { Ampère's law: } & \mathbf{c u r l} \mathbf{h}=i \omega \mathbf{d}+\mathbf{j},
\end{array}
$$

posed over all of affine space $A\left(\mathbb{R}^{3}\right)$. The equations link (the complex amplitudes of) the electric field $\mathbf{e}$, the magnetic induction $\mathbf{b}$, the magnetic field $\mathbf{h}$, and the displacement current $\mathbf{d}$. Here, $\mathbf{j}$ denotes a (formal) excitation supplied by an imposed current. The equations have to be supplemented by the material laws (also called constitutive laws)

$$
\mathbf{d}=\epsilon \mathbf{e}, \quad \mathbf{b}=\mu \mathbf{h},
$$

where the dielectric tensor $\boldsymbol{\epsilon}$ and the magnetic permeability tensor $\boldsymbol{\mu}$ are usually introduced as $L^{\infty}$-functions mapping into the real symmetric, positive definite $3 \times 3$ matrices such that $\lambda_{\min }(\boldsymbol{\epsilon}(\mathbf{x}))>\epsilon_{0}>0$ and $\lambda_{\min }(\boldsymbol{\mu}(\mathbf{x}))>$ $\mu_{0}>0$ almost everywhere. Such matrix-valued functions will be referred to as metric tensors in the following. If good conductors are involved, a part of the source current may be given through Ohm's law

$$
\mathbf{j}=\sigma \mathbf{e}+\mathbf{j}_{0},
$$

where $\boldsymbol{\sigma}$ stands for the symmetric, positive semi-definite conductivity tensor, yet another metric tensor.

\subsection{Fields and forms}

Is there more to the unknowns of (2.1) than being plain vector-fields with three components? To answer this question, it is useful to remember the physicists' favourite way of writing Maxwell's equations, namely the integral form:

$$
\begin{array}{ll}
\text { Faraday's law: } & \int_{\partial \Sigma} \mathbf{e} \cdot \mathrm{d} \mathbf{s}=-i \omega \int_{\Sigma} \mathbf{b} \cdot \mathbf{n} \mathrm{d} S \\
\text { Ampère's law: } & \int_{\partial \Sigma} \mathbf{h} \cdot \mathrm{d} \mathbf{s}=i \omega \int_{\Sigma} \mathbf{d} \cdot \mathbf{n} \mathrm{d} S+\int_{\Sigma} \mathbf{j} \cdot \mathbf{n} \mathrm{d} S .
\end{array}
$$


This is to hold for any bounded, two-dimensional, piecewise smooth submanifold $\Sigma$ of $A\left(\mathbb{R}^{3}\right)$, equipped with oriented ${ }^{1}$ unit normal vector-field $\mathbf{n}$.

First, the integral form (2.4) reveals that the fields $\mathbf{e}, \mathbf{h}$ and $\mathbf{b}, \mathbf{d}$ have an entirely different nature, as Maxwell remarked in his 'Treatise on Electricity and Magnetism' (Maxwell 1891, Chapter 1):

Physical vector quantities may be divided into two classes, in one of which the quantity is defined with reference to a line, while in the other the quantity is defined with reference to an area.

Laconically speaking, electromagnetic fields are an abstraction for associating 'voltages' and 'fluxes' to directed paths and oriented surfaces; they are integral forms in the sense of the following definition, which is deliberately kept fuzzy because it targets some 'intuitive concepts'. ${ }^{2}$

Definition 1. An integral form of degree $l \in \mathbb{N}_{0}, 0 \leq l \leq n, n \in \mathbb{N}$, on a piecewise smooth $n$-dimensional manifold $\mathcal{M}$ is a continuous ${ }^{3}$ additive mapping from the set $\mathcal{S}_{l}(\mathcal{M})$ of compact, oriented, piecewise smooth, $l$ dimensional sub-manifolds of $\mathcal{M}$ into the complex numbers. These so-called integral $l$-forms on $\mathcal{M}$ form the vector space $\mathcal{F}^{l}(\mathcal{M})$ (which is to be trivial for $l<0$ or $l>n$ ).

Here, by 'additive', we mean that the integral form assigns the sum of the respective numbers to the union of disjoint sub-manifolds. Further, flipping the orientation of a sub-manifold should change the sign of the assigned value. This is what we should expect from the integrals occurring in (2.4). Therefore the evaluation $\omega(\Sigma), \omega \in \mathcal{F}^{l}(\mathcal{M}), \Sigma \in \mathcal{S}_{l}(\mathcal{M})$ is dubbed 'integrating $\omega$ over $\Sigma$ ', in symbols $\int_{\Sigma} \omega$. Now, by merely looking at (2.4), we identify $\mathbf{e}$ and $\mathbf{h}$ as integral 1-forms, whereas $\mathbf{b}, \mathbf{d}$, and $\mathbf{j}$ should be regarded as integral 2-forms.

We are accustomed to referring to the 'field at a point in space', that is, a local perspective. Measurement procedures adopt it: measuring an electric field amounts to determining the virtual work

$$
\delta w=q \mathbf{e}(\mathbf{x}) \cdot \delta \mathbf{x}
$$

needed for the tiny displacement $\delta \mathbf{x}$ of a test charge $q$ at $\mathbf{x}$, with $\cdot$ designating the inner product in Euclidean space $\mathbb{R}^{3}$. The magnetic induction is

1 Taking for granted an orientation of the ambient space $A\left(\mathbb{R}^{3}\right)$, we need not distinguish between interior and exterior orientation of manifolds.

${ }^{2}$ It is the subject of geometric measure theory to come up with a more rigorous approach. See Morgan (1995) for an introduction and Federer (1969) for a comprehensive exposition.

${ }^{3}$ Continuity refers to a sort of 'deformation topology' on sets of piecewise smooth manifolds. 
measured through the Lorenz force, that is, the work

$$
\delta w=q(\mathbf{b}(\mathbf{x}) \times \mathbf{v}) \cdot \delta \mathbf{x}
$$

required for a tiny (transversal) shift of a test charge $q$ at $\mathbf{x}$ moving with velocity $\mathbf{v}$, where $\times$ is the usual cross product of vectors in $\mathbb{R}^{3}$. From this perspective $\mathbf{e}$ and $\mathbf{b}$ are classical (continuous) differential forms of degree 1 and 2, respectively, according to the following definition (cf. Lang (1995, Chapter V, Section 3)).

Definition 2. A differential form of degree $l, l \in \mathbb{N}_{0}$, and class $C^{m}, m \in$ $\mathbb{N}_{0}$, on a smooth $n$-manifold $\mathcal{M}$ is an $m$-times continuously differentiable mapping assigning to each $\mathbf{x} \in \mathcal{M}$ an element of the space $\Lambda^{l}\left(T_{\mathcal{M}}(\mathbf{x})\right)$ of alternating $l$-multilinear forms on the tangent space $T_{\mathcal{M}}(\mathbf{x})$. These mappings form the vector space $\mathcal{D} \mathcal{F}^{l, m}(\mathcal{M})$.

Any piecewise smooth oriented manifold can be covered and approximated arbitrarily well by tiny flat 'tangential' tiles. Thus, through Riemann summation any differential $l$-form spawns an integral $l$-form according to Definition 1 ( $c f$. Bossavit (1998d, Section 3.2)). This gives us injections $F_{\mathcal{M}}^{l}: \mathcal{D F}^{l, 0}(\mathcal{M}) \mapsto \mathcal{F}^{l}(\mathcal{M})$, which, of course, are by no means surjective.

A special case is $\mathcal{M}=A\left(\mathbb{R}^{3}\right)$. Then $T_{\mathcal{M}}(\mathbf{x})=\mathbb{R}^{3}$ for all $\mathbf{x} \in \mathcal{M}$ and $T_{\mathcal{M}}(\mathbf{x})$ may be endowed with the structure of a Euclidean vector space. Then the identifications of Table 2.1 establish isomorphisms $\Upsilon_{l}$ between differential $l$-forms, $0 \leq l \leq 3$, on $A\left(\mathbb{R}^{3}\right)$ and continuous functions/vector-fields, their vector proxies (a term coined by A. Bossavit (1998e)).

Using the convention put forth in Table 2.1, the integration of forms amounts to the evaluation of the following integrals for vector proxies:

$$
\begin{array}{rlrl}
\omega \in \mathcal{D F}^{0,0}(\Omega): & \int_{\mathbf{x}} \omega=\left(\Upsilon_{0} \omega\right)(\mathbf{x}) & & \forall \mathbf{x} \in \Omega, \\
\omega \in \mathcal{D F}^{1,0}(\Omega): & \int_{\gamma} \omega=\int_{\gamma} \Upsilon_{1} \omega \cdot \mathrm{d} \mathbf{s} & & \forall \gamma \in \mathcal{S}_{1}(\Omega), \\
\omega \in \mathcal{D F}^{2,0}(\Omega): & \int_{\Sigma} \omega=\int_{\Sigma} \Upsilon_{2} \omega \cdot \mathbf{n} \mathrm{d} S & & \forall \Sigma \in \mathcal{S}_{2}(\Omega), \\
\omega \in \mathcal{D F}^{3,0}(\Omega): & \int_{V} \omega=\int_{V} \Upsilon_{3} \omega \mathrm{d} \mathbf{x} & \forall V \in \mathcal{S}_{3}(\Omega) .
\end{array}
$$

Here, $\mathbf{n}$ is a unit normal vector-field to $\Sigma$ whose direction is induced by the orientation of $\Sigma$.

It is clear that we have a lot of freedom when defining vector proxies. Choosing an inner product for $\mathbb{R}^{3}$ gives us other vector proxies for the same differential forms. In short, vector proxies are coordinate-dependent, in contrast to the calculus of differential forms.

More generally, the finite-dimensional spaces $\bigwedge^{l}\left(T_{\mathcal{M}}(\mathbf{x})\right)$ can be equipped with a basis generated by coordinate vectors of charts of $\mathcal{M}$. Thus, any differential $l$-form on $\mathcal{M}$ can be identified with the $\left(\begin{array}{l}n \\ l\end{array}\right)$-tuple of its coefficient 
Table 2.1. Relationship between differential forms and vector-fields in three-dimensional Euclidean space $\left(\mathbf{v}, \mathbf{v}_{1}, \mathbf{v}_{2}, \mathbf{v}_{3} \in \mathbb{R}^{3}\right)$.

\begin{tabular}{ll}
\hline \hline Differential form & Related function $u /$ vector-field $\mathbf{u}$ \\
\hline $\mathbf{x} \mapsto \omega(\mathbf{x})$ & $u(\mathbf{x}):=\omega(\mathbf{x})$ \\
$\mathbf{x} \mapsto\{\mathbf{v} \mapsto \omega(\mathbf{x})(\mathbf{v})\}$ & $\langle\mathbf{u}(\mathbf{x}), \mathbf{v}\rangle:=\omega(\mathbf{x})(\mathbf{v})$ \\
$\mathbf{x} \mapsto\left\{\left(\mathbf{v}_{1}, \mathbf{v}_{2}\right) \mapsto \omega(\mathbf{x})\left(\mathbf{v}_{1}, \mathbf{v}_{2}\right)\right\}$ & $\left\langle\mathbf{u}(\mathbf{x}), \mathbf{v}_{1} \times \mathbf{v}_{2}\right\rangle:=\omega(\mathbf{x})\left(\mathbf{v}_{1}, \mathbf{v}_{2}\right)$ \\
$\mathbf{x} \mapsto\left\{\left(\mathbf{v}_{1}, \mathbf{v}_{2}, \mathbf{v}_{3}\right) \mapsto \omega(\mathbf{x})\left(\mathbf{v}_{1}, \mathbf{v}_{2}, \mathbf{v}_{3}\right)\right\}$ & $u(\mathbf{x}) \operatorname{det}\left(\mathbf{v}_{1}, \mathbf{v}_{2}, \mathbf{v}_{3}\right):=\omega(\mathbf{x})\left(\mathbf{v}_{1}, \mathbf{v}_{2}, \mathbf{v}_{3}\right)$ \\
\hline
\end{tabular}

functions with respect to the bases. Often, calculations with differential forms are greatly facilitated by using a coefficient representation. There is absolutely no objection to using vector proxies as long as their use is consistent with the physical meaning of the fields and confined to legal operations for integral forms. For example, point evaluations of vector proxies of 1 -forms should be used with great care, since they fail to make sense for integral 1-forms.

\section{Bibliographical notes}

The interpretation of electromagnetic fields as differential forms has a long tradition in mathematical physics and is covered in many textbooks on differential forms. Some references include Grauert and Lieb (1977, Chapter 5), Baldomir and Hammond (1996), and Burke (1985, Chapter VI). This last reference gives lucid explanations for the concept from differential geometry used in this section. Brief presentations of the topic include Bossavit (1998b, 1998c), Deschamps (1981) and Baldomir (1986). The role of the integral conservation form of Maxwell's equations in space and time is a core theme in Mattiussi (2000).

\subsection{Exterior calculus}

Prominent in the integral formulation of Maxwell's equations is the evaluation of integral 1-forms over boundaries. This motivates the definition of $e x$ terior derivatives: these are linear operators $\boldsymbol{d}: \mathcal{F}^{l}(\mathcal{M}) \mapsto \mathcal{F}^{l+1}(\mathcal{M})$, where $\mathcal{M}$ is a piecewise smooth orientable $n$-dimensional manifold, $0 \leq l<n$, defined by

$$
\int_{\Sigma} d \omega:=\int_{\partial \Sigma} \omega, \quad \text { for all } \omega \in \mathcal{F}^{l}(\mathcal{M}), \Sigma \in \mathcal{S}_{l+1}(\mathcal{M}) .
$$

The boundary $\partial \Sigma$ bears the induced orientation, and for $\omega \in \mathcal{F}^{n}(\mathcal{M})$ we 
set $\boldsymbol{d} \omega=0$. Forms in the kernel of $\boldsymbol{d}$ are called closed. It goes without saying that $\partial \partial \Sigma=\emptyset$, which immediately implies the fundamental relation $\boldsymbol{d} \circ \boldsymbol{d}=0$. The converse of this statement is the core of the famous Poincaré lemma. There is too little structure in integral forms as we have introduced them to support a proof, but this fundamental lemma expresses that

$$
\text { ' } \omega \in \mathcal{F}^{l}(\mathcal{M}): \quad \boldsymbol{d} \omega=0 \quad \Leftrightarrow \quad \exists \eta \in \mathcal{F}^{l-1}(\mathcal{M}): \quad \omega=\boldsymbol{d} \eta ',
$$

if $\mathcal{M}$ is homeomorphic to an $n$-ball. We will just assume this to hold in the intuitive setting of integral forms.

In Section 2.1 we concluded that the electromagnetic fields can be modelled through integral forms. Hence, the notion of an exterior derivative permits us to recast the integral form (2.4) of Maxwell's equations as

$$
\boldsymbol{d e}=-i \omega \mathbf{b}, \quad \boldsymbol{d} \mathbf{h}=i \omega \mathbf{d}+\mathbf{j} .
$$

The statement of (2.9) only relies on the topological concepts of orientation and boundaries of manifolds. Therefore, the relationships in (2.9) may be called topological laws.

Two conclusions can be drawn from (2.9) and (2.8). First, from $\boldsymbol{d} \circ \boldsymbol{d}=0$ we get the conservation laws

$$
\boldsymbol{d} \mathbf{b}=0, \quad \boldsymbol{d}(i \omega \mathbf{d}+\mathbf{j})=0 .
$$

Second, as (2.9) holds on all of $A\left(\mathbb{R}^{3}\right)$, by (2.8) we can find a magnetic vector potential $\mathbf{a} \in \mathcal{F}^{1}\left(A\left(\mathbb{R}^{3}\right)\right)$ and a scalar potential $v \in \mathcal{F}^{0}\left(A\left(\mathbb{R}^{3}\right)\right)$ such that

$$
\mathbf{b}=\boldsymbol{d} \mathbf{a}, \quad \mathbf{e}=-\boldsymbol{d} v-i \omega \boldsymbol{d} \mathbf{a} .
$$

A very natural concept is that of the transformation of integral forms under a diffeomorphism $\boldsymbol{\Phi}: \widehat{\mathcal{M}} \mapsto \mathcal{M}$ of manifolds, the so-called pullback $\mathbf{\Phi}^{*}$ : $\mathcal{F}^{l}(\mathcal{M}) \mapsto \mathcal{F}^{l}(\widehat{\mathcal{M}})$ defined by

$$
\boldsymbol{\Phi}^{*} \omega \in \mathcal{F}^{l}(\widehat{\mathcal{M}}): \quad \int_{\widehat{\Sigma}} \boldsymbol{\Phi}^{*} \omega=\int_{\boldsymbol{\Phi}(\widehat{\Sigma})} \omega \quad \forall \widehat{\Sigma} \in \mathcal{S}_{l}(\widehat{\mathcal{M}}) .
$$

Straight from the definitions (2.8) and (2.12) we infer that the exterior derivative and pullback commute, that is,

$$
d \circ \Phi^{*}=\Phi^{*} \circ d
$$

This carries the important consequence that, if integral forms satisfy the topological laws (2.9) on some domain $\Omega \subset A\left(\mathbb{R}^{3}\right)$, then their pullbacks will satisfy the same relationships on a transformed domain. In short, the topological laws are invariant under diffeomorphic transformations. 
The trace $\mathbf{t}_{\mathcal{N}} \omega$ of a form $\omega \in \mathcal{F}^{l}(\mathcal{M})$ onto a sub-manifold $\mathcal{N} \in \mathcal{S}_{m}(\mathcal{M})$, $0 \leq m \leq n$, is straightforward:

$$
\int_{\Sigma} \mathbf{t}_{\mathcal{N}} \omega:=\int_{\Sigma} \omega \quad \forall \Sigma \in \mathcal{S}_{l}(\mathcal{N}) .
$$

It is clear that the trace commutes with both the exterior derivative and the pullback, that is, $\boldsymbol{d} \circ \mathbf{t}_{\mathcal{N}}=\mathbf{t}_{\mathcal{N}} \circ \boldsymbol{d}$ and $\boldsymbol{\Phi}^{*} \circ \mathbf{t}_{\mathcal{N}}=\mathbf{t}_{\widehat{\mathcal{N}}} \circ \Phi^{*}$.

Traces give a meaning to boundary conditions for electromagnetic fields: imposing boundary conditions amounts to fixing the trace of a field on some surfaces. Though a genuine boundary does not exist in electrodynamics, it is often convenient to assume that fields cannot penetrate some surfaces. This is reflected either by the perfect electric conductor (PEC) boundary conditions $\mathbf{t}_{\Sigma} \mathbf{e}=0$ on $\Sigma \in \mathcal{S}_{2}\left(A\left(\mathbb{R}^{3}\right)\right)$ or magnetic wall boundary conditions $(\mathrm{PMC}) \mathbf{t}_{\Sigma} \mathbf{h}=0$. Thus, using the trace of 1 -forms gives a clear hint about meaningful boundary conditions for electromagnetic fields.

It is the feat of exterior calculus in differential geometry to establish a meaning of the exterior derivative, the pullback, and the trace for differential forms such that the diagrams

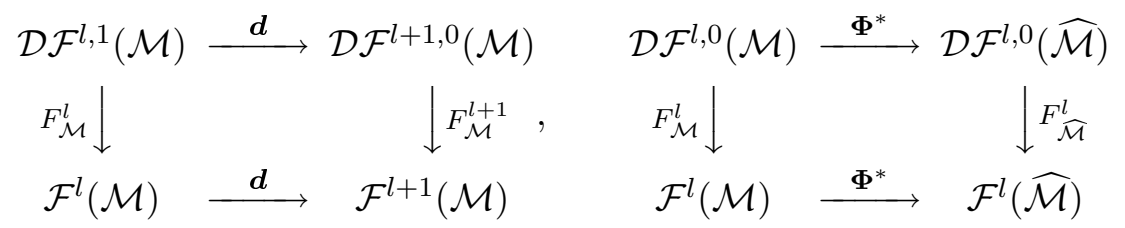

commute. Here, we have used the same symbols for the new operators on differential forms. Given local representations of $\boldsymbol{d}, \boldsymbol{\Phi}^{*}$, and $\mathbf{t}_{\mathcal{N}}$, and the associations of Table 2.1, it merely takes technical manipulations to come up with incarnations for vector proxies in the case of differential forms defined on a domain $\Omega \subset A\left(\mathbb{R}^{3}\right)$. One finds, based on the identifications of Table 2.1,

$$
\Upsilon_{1} \circ \boldsymbol{d}=\operatorname{grad} \circ \Upsilon_{0}, \Upsilon_{2} \circ \boldsymbol{d}=\operatorname{curl} \circ \Upsilon_{1}, \Upsilon_{3} \circ \boldsymbol{d}=\operatorname{div} \circ \Upsilon_{2} .
$$

This establishes the link between (2.1) and (2.9). The pullbacks, when considered for vector proxies of continuous differential forms, give rise to familiar transformations:

$$
\begin{array}{ll}
\mathfrak{F}_{\mathbf{\Phi}}^{0}:=\Upsilon_{0} \circ \boldsymbol{\Phi}^{*} \circ \Upsilon_{0}^{-1}, & \left(\mathfrak{F}_{\mathbf{\Phi}}^{0} u\right)(\widehat{\mathbf{x}})=u(\mathbf{x}), \\
\mathfrak{F}_{\mathbf{\Phi}}^{1}:=\Upsilon_{1} \circ \boldsymbol{\Phi}^{*} \circ \Upsilon_{1}^{-1}, & \left(\mathfrak{F}_{\mathbf{\Phi}}^{1} \mathbf{u}\right)(\widehat{\mathbf{x}})=D \boldsymbol{\Phi}(\widehat{\mathbf{x}})^{T} \mathbf{u}(\mathbf{x}), \\
\mathfrak{F}_{\mathbf{\Phi}}^{2}:=\Upsilon_{2} \circ \boldsymbol{\Phi}^{*} \circ \Upsilon_{2}^{-1}, & \left(\mathfrak{F}_{\mathbf{\Phi}}^{2} \mathbf{u}\right)(\widehat{\mathbf{x}})=\operatorname{det} D \boldsymbol{\Phi}(\widehat{\mathbf{x}}) D \boldsymbol{\Phi}(\widehat{\mathbf{x}})^{-1} \mathbf{u}(\mathbf{x}), \\
\mathfrak{F}_{\mathbf{\Phi}}^{3}:=\Upsilon_{3} \circ \boldsymbol{\Phi}^{*} \circ \Upsilon_{3}^{-1}, & \left(\mathfrak{F}_{\mathbf{\Phi}}^{3} u\right)(\widehat{\mathbf{x}})=\operatorname{det} D \boldsymbol{\Phi}(\widehat{\mathbf{x}}) u(\mathbf{x}) .
\end{array}
$$

Here $u$ stands for a continuous function on $\Omega, \mathbf{u}$ for a continuous vector-field with three components, $\boldsymbol{\Phi}: \widehat{\Omega} \mapsto \Omega$ is a diffeomorphism, $D \boldsymbol{\Phi}$ its Jacobian, and $\widehat{\mathbf{x}} \in \widehat{\Omega}, \mathbf{x}:=\boldsymbol{\Phi}(\widehat{\mathbf{x}})$. 
Finally, the trace of continuous vector proxies onto an oriented, piecewise smooth 2-dimensional sub-manifold $\Gamma$ of $\Omega$ generates the following formulae:

$$
\begin{aligned}
\gamma:=\Upsilon_{0}^{\Gamma} \circ \mathbf{t}_{\Gamma} \circ \Upsilon_{0}^{-1}, & & (\gamma u)(\mathbf{x})=u(\mathbf{x}), \\
\gamma_{\mathbf{t}}:=\Upsilon_{1}^{\Gamma} \circ \mathbf{t}_{\Gamma} \circ \Upsilon_{1}^{-1}, & & \left(\gamma_{\mathbf{t}} \mathbf{u}\right)(\mathbf{x})=\mathbf{n}(\mathbf{x}) \times(\mathbf{u}(\mathbf{x}) \times \mathbf{n}(\mathbf{x})), \\
\gamma_{\mathbf{n}}:=\Upsilon_{2}^{\Gamma} \circ \mathbf{t}_{\Gamma} \circ \Upsilon_{2}^{-1}, & & \left(\gamma_{\mathbf{n}} \mathbf{u}\right)(\mathbf{x})=\mathbf{u}(\mathbf{x}) \cdot \mathbf{n}(\mathbf{x}) .
\end{aligned}
$$

for $\mathbf{x}$ in smooth components of $\Gamma$. As usual, $\mathbf{n}(\mathbf{x})$ is a unit normal vectorfield whose direction is prescribed by the (external) orientation of $\Gamma$. We point out that $\Upsilon_{0}^{\Gamma}, \Upsilon_{1}^{\Gamma}$, and $\Upsilon_{2}^{\Gamma}$ are isomorphisms asscociating vector proxies to forms on the two-dimensional manifold $\Gamma$. These mappings are chosen based on 'projected Euclidean coordinates'. Here, we skip the details.

The traces of differential forms will be important for a particular reason. Consider $\Omega \subset A\left(\mathbb{R}^{3}\right)$ split into two subdomains $\Omega_{1}$ and $\Omega_{2}$ separated by a piecewise smooth, oriented interface $\Gamma$. When will $\omega \in \mathcal{D F}^{l, 0}\left(\bar{\Omega}_{1}\right) \times$ $\mathcal{D} \mathcal{F}^{l, 0}\left(\bar{\Omega}_{2}\right)$ give rise to a valid integral $l$-form on all of $\Omega$ ? The answer is the patch condition

$$
\omega \in \mathcal{F}^{l}(\Omega) \quad \Leftrightarrow \quad \mathbf{t}_{\Gamma} \omega_{\mid \Omega_{1}}=\mathbf{t}_{\Gamma} \omega_{\mid \Omega_{2}} \quad \text { on } \Gamma .
$$

It translates into the requirement of continuity, tangential continuity, and normal continuity as suitable patch conditions for vector proxies of 0 -forms, 1-forms, and 2-forms, respectively. From (2.20) it is clear what the transmission conditions for electromagnetic fields must look like. These have to make sure that they make sense as global integral forms. Thus, across any piecewise smooth oriented surface $\Sigma$, the traces of both $\mathbf{e}$ and $\mathbf{h}$ must be continuous. Denoting by $[\cdot]_{\Sigma}$ the difference of traces from both sides (the jump), we find, in terms of vector proxies,

$$
\left[\gamma_{\mathbf{t}} \mathbf{e}\right]_{\Sigma}=0, \quad\left[\gamma_{\mathbf{t}} \mathbf{h}\right]_{\Sigma}=0
$$

Eventually, in the calculus of differential forms, the Poincaré lemma can be stated as a theorem. In fact, it becomes part of a celebrated, far more general result.

Theorem 2.1. (DeRham theorem for differential forms) There is a finite-dimensional subspace $\mathcal{D} \mathcal{H}^{l, 0}(\mathcal{M}) \subset \mathcal{D} \mathcal{F}^{l, 0}(\mathcal{M})$ of closed differential $l$ forms, whose dimension is equal to the $l$ th Betti number of $\Omega$, such that, for $\omega \in \mathcal{D} \mathcal{F}^{l, 0}(\mathcal{M})$, we have

$$
\boldsymbol{d} \omega=0 \quad \Leftrightarrow \quad \exists \eta \in \mathcal{D F}^{l-1,1}(\mathcal{M}), \tau \in \mathcal{D H}^{l, 0}(\mathcal{M}) \quad \text { satisfying } \omega=\boldsymbol{d} \eta+\tau .
$$

So far we have not gone much beyond operations already defined for integral forms. Now, we introduce an important device based on a local viewpoint. It is the exterior product, a bilinear mapping

$$
\wedge: \mathcal{D} \mathcal{F}^{l, 0}(\mathcal{M}) \times \mathcal{D} \mathcal{F}^{m, 0}(\mathcal{M}) \mapsto \mathcal{D F} \mathcal{F}^{l+m, 0}(\mathcal{M})
$$


pointwise defined via the $\wedge$-product of alternating multilinear forms. It is connected with the other operations through

$$
\begin{aligned}
& \omega \wedge \eta=(-1)^{l m}(\eta \wedge \omega) \quad \forall \omega \in \mathcal{D F}^{l, 0}(\mathcal{M}), \eta \in \mathcal{D F}^{m, 0}(\mathcal{M}), \\
& \boldsymbol{d}(\omega \wedge \eta)=\boldsymbol{d} \omega \wedge \eta+(-1)^{l}(\omega \wedge \boldsymbol{d} \eta) \quad \forall \omega \in \mathcal{D F}^{l, 1}(\mathcal{M}), \eta \in \mathcal{D F}^{m, 1}(\mathcal{M}), \\
& \boldsymbol{\Phi}^{*}(\omega \wedge \eta)=\boldsymbol{\Phi}^{*} \omega \wedge \boldsymbol{\Phi}^{*} \eta \quad \forall \omega \in \mathcal{D F}^{l, 0}(\mathcal{M}), \eta \in \mathcal{D} \mathcal{F}^{m, 0}(\mathcal{M}) .
\end{aligned}
$$

The second equation combined with the definition of the exterior derivative yields the vital integration by parts formula

$$
\int_{\Sigma} \boldsymbol{d} \omega \wedge \eta+(-1)^{l}(\omega \wedge \boldsymbol{d} \eta)=\int_{\partial \Sigma} \omega \wedge \eta
$$

for $\omega \in \mathcal{D} \mathcal{F}^{l, 0}(\mathcal{M}), \eta \in \mathcal{D} \mathcal{F}^{m, 0}(\mathcal{M}), \Sigma \in \mathcal{S}_{l+m+1}(\mathcal{M})$. In terms of Euclidean vector proxies, the exterior product reads

$$
\begin{array}{ll}
\Upsilon_{2}(\omega \wedge \eta)=\left(\Upsilon_{1} \omega\right) \times\left(\Upsilon_{1} \eta\right) & \forall \omega, \eta \in \mathcal{D} \mathcal{F}^{1,0}(\Omega), \\
\Upsilon_{3}(\omega \wedge \eta)=\left(\Upsilon_{2} \omega\right) \cdot\left(\Upsilon_{1} \eta\right) & \forall \omega \in \mathcal{D} \mathcal{F}^{2,0}(\Omega), \eta \in \mathcal{D} \mathcal{F}^{1,0}(\Omega) .
\end{array}
$$

An exterior product with a 0 -form amounts to a pointwise multiplication with its related function. These relationships supply the customary integration by parts formulas (Green's formulae) for functions and vector-fields.

Remark 1. The perspective of differential forms rewards us with insights into hidden relationships. Just tinker with the order of forms in the topological laws (2.9) by viewing $\mathbf{e}$ as a 0 -form, $\mathbf{b}$ as a 1 -form, $\mathbf{h}$ as a 2 -form, and $\mathbf{d}, \mathbf{j}$ as 3 -forms. This makes perfect sense and we recover the topological laws underlying the Helmholtz equation of linear acoustics, because in terms of vector proxies we get

$$
\operatorname{grad}^{\prime} \mathbf{e}^{\prime}=-i \omega^{\prime} \mathbf{b} ', \quad \operatorname{div} \text { 'h' }=i \omega^{\prime} \mathbf{d} \text { ' }+ \text { 'j'. }
$$

This looks odd, but now e must be seen as a scalar potential (pressure) and $\mathbf{h}$ is usually called the flux. Hence Maxwell's equations are a member of a larger family of models, to which the acoustic wave equation belongs as well. Please be aware that, in terms of differential forms, it is related to the scalar wave equation and not to the vectorial one. We also realize a significant difference between the models for acoustics and electromagnetism. In the latter case both $\mathbf{e}$ and $\mathbf{h}$ are 1 -forms, which hints at a fundamental symmetry.

\section{Bibliographical notes}

The theory of differential forms is a classical branch of differential geometry covered by many textbooks, of which I would like to mention Cartan (1967). This is the main reference for all results cited above, besides Burke (1985, Chapter IV) and Lang (1995, Chapter V). A lucid presentation is given 
in Bossavit (1998d). There is a close link with algebraic topology, of which Bott and $\mathrm{Tu}(1982)$ give a substantial account.

\subsection{Variational formulations}

So far we have evaded the vexing question of how the material laws fit the framework of forms that we have embraced in the previous sections. Evidently, they link forms of different order, and therefore the multiplications with $\boldsymbol{\epsilon}, \boldsymbol{\mu}$, and $\boldsymbol{\sigma}$ must be regarded as special linear operators.

Keep in mind that the material laws arise from averaging microscopic effects. In a sense they are less fundamental than the topological laws (2.9), and make sense only on a macroscopic scale. The material laws introduce the concept of field energy into the model. Let $E_{\text {el }}(\mathbf{e})$ denote the energy contained in the electric field within a bounded control volume $\Omega \subset A\left(\mathbb{R}^{3}\right)$. Since we admit only linear materials, $E_{\mathrm{el}}$ is a quadratic form, which arises from a symmetric positive definite sesqui-linear form $a_{\epsilon}$ by $E_{\text {el }}(\mathbf{v})=\frac{1}{2} a_{\boldsymbol{\epsilon}}(\mathbf{v}, \mathbf{v})$ for all $\mathbf{v} \in \mathcal{F}^{1}(\Omega)$. Then the displacement current $\mathbf{d}$ has to satisfy

$$
\int_{\Omega} \mathbf{d} \wedge \overline{\mathbf{e}}^{\prime}=a_{\boldsymbol{\epsilon}}\left(\mathbf{e}, \mathbf{e}^{\prime}\right) \quad \forall \mathbf{e}^{\prime} \in \mathcal{D} \mathcal{F}^{1,0}(\Omega),
$$

where an over-bar indicates the complex conjugate. In fact, $\mathbf{d}$ could be regarded as a linear form on the space of differential 1-forms, that is, a 1current (Grauert and Lieb 1977, Chapter 5). Yet we will not pursue this, and continue viewing $\mathbf{d}$ as a 2 -form.

Similarly, the magnetic induction $\mathbf{b}$ possesses the magnetic energy $E_{\text {mag }}$ on $\Omega$. It is related to a symmetric, positive definite sesqui-linear form $a_{1 / \boldsymbol{\mu}}$ by $E_{\text {mag }}(\mathbf{v})=\frac{1}{2} a_{1 / \boldsymbol{\mu}}(\mathbf{v}, \mathbf{v})$ for all $\mathbf{v} \in \mathcal{F}^{2}(\Omega)$. Then the magnetic field $\mathbf{h}$ has to fulfil

$$
\int_{\Omega} \mathbf{h} \wedge \overline{\mathbf{b}}^{\prime}=a_{1 / \boldsymbol{\mu}}\left(\mathbf{b}, \mathbf{b}^{\prime}\right) \quad \forall \mathbf{b}^{\prime} \in \mathcal{D} \mathcal{F}^{2,0}(\Omega) .
$$

As the exterior product introduces a non-degenerate pairing, (2.24) and (2.25) also assign energies to the fields $\mathbf{h}$ and $\mathbf{d}$. Thus we may introduce symmetric, positive definite sesqui-linear forms $a_{\boldsymbol{\mu}}$ and $a_{1 / \boldsymbol{\epsilon}}$ and express

$$
\begin{gathered}
\int_{\Omega} \mathbf{b} \wedge \overline{\mathbf{h}}^{\prime}=a_{\boldsymbol{\mu}}\left(\mathbf{h}, \mathbf{h}^{\prime}\right) \quad \forall \mathbf{h}^{\prime} \in \mathcal{D} \mathcal{F}^{1,0}(\Omega), \\
\int_{\Omega} \mathbf{e} \wedge \overline{\mathbf{d}}^{\prime}=a_{1 / \boldsymbol{\epsilon}}\left(\mathbf{d}, \mathbf{d}^{\prime}\right) \quad \forall \mathbf{d}^{\prime} \in \mathcal{D} \mathcal{F}^{2,0}(\Omega) .
\end{gathered}
$$

The analogous treatment of Ohm's law (2.3) is left to the reader. 
The material laws in variational form can be combined with the topological laws and lead to natural weak formulations of Maxwell's equations. We can distinguish between two essentially distinct approaches. They differ in how the two topological laws are taken into account. On the one hand, Ampère's law, when tested with $\mathbf{e}^{\prime} \in \mathcal{D F}^{1,0}(\bar{\Omega})$ on a bounded control volume $\Omega$, gives rise to

$$
\int_{\Omega} \boldsymbol{d} \mathbf{h} \wedge \overline{\mathbf{e}}^{\prime}=i \omega \int_{\Omega} \mathbf{d} \wedge \overline{\mathbf{e}}^{\prime}+\int_{\Omega} \mathbf{j} \wedge \overline{\mathbf{e}}^{\prime}
$$

Now, integration by parts is performed according to (2.23), which means that Ampère's law enters in a weak sense only:

$$
\int_{\Omega} \mathbf{h} \wedge \boldsymbol{d} \overline{\mathbf{e}}^{\prime}+\int_{\partial \Omega} \mathbf{h} \wedge \overline{\mathbf{e}}^{\prime}=i \omega \int_{\Omega} \mathbf{d} \wedge \overline{\mathbf{e}}^{\prime}+\int_{\Omega} \mathbf{j} \wedge \overline{\mathbf{e}}^{\prime} .
$$

Two integrals in this equation can be replaced by means of (2.24) and (2.25), which yields

$$
a_{1 / \boldsymbol{\mu}}\left(\mathbf{b}, \boldsymbol{d} \mathbf{e}^{\prime}\right)+\int_{\partial \Omega} \mathbf{h} \wedge \overline{\mathbf{e}}^{\prime}=i \omega a_{\boldsymbol{\epsilon}}\left(\mathbf{e}, \mathbf{e}^{\prime}\right)+\int_{\Omega} \mathbf{j} \wedge \overline{\mathbf{e}}^{\prime} \quad \forall \mathbf{e}^{\prime} \in \mathcal{D} \mathcal{F}^{1,0}(\Omega) .
$$

Next, Faraday's law is used to express b through $\boldsymbol{d e}$, and we end up with the 'e-based' primal variational formulation of Maxwell's equations: the electric field solution e satisfies

$$
a_{1 / \boldsymbol{\mu}}\left(\boldsymbol{d e}, \boldsymbol{d \mathbf { e } ^ { \prime }}\right)-\omega^{2} a_{\boldsymbol{\epsilon}}\left(\mathbf{e}, \mathbf{e}^{\prime}\right)-i \omega \int_{\partial \Omega} \mathbf{h} \wedge \overline{\mathbf{e}}^{\prime}=-i \omega \int_{\Omega} \mathbf{j} \wedge \overline{\mathbf{e}}^{\prime}
$$

for all $\mathbf{e}^{\prime} \in \mathcal{D F}^{1,0}(\Omega)$. On the other hand, we may choose to take into account Faraday's law, in weak form, by

$$
\int_{\Omega} \mathbf{e} \wedge d \overline{\mathbf{h}}^{\prime}-\int_{\partial \Omega} \mathbf{e} \wedge \overline{\mathbf{h}}^{\prime}=-i \omega \int_{\Omega} \mathbf{b} \wedge \overline{\mathbf{h}}^{\prime} \quad \forall \mathbf{h}^{\prime} \in \mathcal{D} \mathcal{F}^{1,0}(\Omega) .
$$

The alternative variational version of the material laws, namely (2.26) and (2.27), have to be used in this case. In addition, $\mathbf{d}$ can be replaced using the strong form of Ampère's law. We arrive at the 'h-based' dual variational formulation: for the magnetic field solution $\mathbf{h}$ we have

$$
a_{1 / \boldsymbol{\epsilon}}\left(\boldsymbol{d} \mathbf{h}, \boldsymbol{d} \mathbf{h}^{\prime}\right)-\omega^{2} a_{\boldsymbol{\mu}}\left(\mathbf{h}, \mathbf{h}^{\prime}\right)+i \omega \int_{\partial \Omega} \mathbf{e} \wedge \overline{\mathbf{h}}^{\prime}=a_{1 / \boldsymbol{\epsilon}}\left(\mathbf{j}, \boldsymbol{d} \mathbf{h}^{\prime}\right)
$$

for all $\mathbf{h}^{\prime} \in \mathcal{D} \mathcal{F}^{1,0}(\Omega)$. To get valid boundary value problems on bounded domains, the boundary terms in both (2.28) and (2.29) have to be dealt with by imposing suitable boundary conditions. For instance, in the case of (2.28) PEC boundary conditions must be imposed strongly and honoured by 
demanding that $\mathbf{t}_{\partial \Omega} \mathbf{e}^{\prime}=0$. Conversely, PMC boundary conditions $\mathbf{t}_{\partial \Omega} \mathbf{h}=0$ are taken into account weakly by dropping the boundary term. For the dual variational problem the handling of boundary conditions is reversed.

Remark 2. The typical situation in computational electromagnetism is marked by unbounded domains. In an abstract way the unbounded exterior of $\Omega$ can be taken into account by replacing

$$
\int_{\partial \Omega} \mathbf{h} \wedge \overline{\mathbf{e}}^{\prime} \longrightarrow \int_{\partial \Omega} \mathrm{S}_{\mathbf{e}} \mathbf{e} \wedge \overline{\mathbf{e}}^{\prime}
$$

in (2.28), where $\mathbf{S}_{\mathbf{e}}$ is the Poincaré-Steklov operator for the exterior electromagnetic field problem (with radiation conditions at $\infty$ ). The PoincaréSteklov operator can be expressed through boundary integral equations. If $\Omega$ is a ball, techniques based on expansions into surface spherical harmonics are available. A survey is given in Nédélec (2001). The numerical treatment of field problems on unbounded exterior domains (scattering problems) is a core area of computational electromagnetism, but will not be covered in this article.

Inherent in the field model is idealization that the field energy can be strictly localized in terms of an energy density. Assuming some smoothness of the fields and letting the control volume $\Omega$ shrink to zero, we finally obtain positive definite quadratic forms on $\bigwedge^{l}\left(\mathbb{R}^{3}\right)\left(l=1\right.$ for $a_{\boldsymbol{\epsilon}}, a_{1 / \boldsymbol{\mu}}, l=2$ for $\left.a_{1 / \boldsymbol{\epsilon}}, a_{\boldsymbol{\mu}}\right)$ for any point $\mathbf{x} \in A\left(\mathbb{R}^{3}\right)$. By simple linear algebra, these define operators $\star: \bigwedge^{l}\left(\mathbb{R}^{3}\right) \mapsto \bigwedge^{3-l}\left(\mathbb{R}^{3}\right)$, which give rise to Hodge operators $\star: \mathcal{D F}^{l, 0}\left(A\left(\mathbb{R}^{3}\right)\right) \mapsto \mathcal{D} \mathcal{F}^{3-l, 0}\left(A\left(\mathbb{R}^{3}\right)\right)$. For vector proxies in Euclidean space $\mathbb{R}^{3}$ they take the form of the conventional material laws (2.2). This will be enough for our purposes and we are not going to dwell on Hodge operators any further. In short, through the notion of local energy densities we find the conventional expressions

$$
\begin{array}{rlrl}
a_{\boldsymbol{\epsilon}}(\mathbf{u}, \mathbf{v}) & =\int_{\Omega} \boldsymbol{\epsilon} \Upsilon_{1} \mathbf{u} \cdot \Upsilon_{1} \mathbf{v} \mathrm{d} \mathbf{x}, & a_{1 / \boldsymbol{\mu}}(\mathbf{u}, \mathbf{v}) & =\int_{\Omega} \boldsymbol{\mu}^{-1} \Upsilon_{2} \mathbf{u} \cdot \Upsilon_{2} \mathbf{v} \mathrm{d} \mathbf{x}, \\
a_{1 / \boldsymbol{\epsilon}}(\mathbf{u}, \mathbf{v}) & =\int_{\Omega} \epsilon^{-1} \Upsilon_{2} \mathbf{u} \cdot \Upsilon_{2} \mathbf{v} \mathrm{d} \mathbf{x}, & a_{\boldsymbol{\mu}}(\mathbf{u}, \mathbf{v})=\int_{\Omega} \boldsymbol{\mu} \Upsilon_{1} \mathbf{u} \cdot \Upsilon_{1} \mathbf{v} \mathrm{d} \mathbf{x},
\end{array}
$$

where $\mathbf{u}, \mathbf{v}$ are forms of appropriate degree. By their derivation these sesquilinear forms are invariant with respect to the choice of vector proxies, because a change of basis also entails a transformation of the metric tensors.

Remark 3. The terminology 'primal' and 'dual' is borrowed from the study of weak formulations of second-order elliptic problems ( $c f$. Brezzi and Fortin (1991, Chapter 1)), that is, the case discussed in Remark 1 where 
e is a 0 -form. Then $\boldsymbol{d}$ becomes grad in the primal variational formulation and the remaining unknown is a plain function. For the dual problem, $\boldsymbol{d}$ is div and the variational formulation is posed for a flux field. In this case the striking difference between the two formulations justifies the labels 'primal' and 'dual' (which stem from convex analysis). In light of the symmetry between $\mathbf{e}$ and $\mathbf{h}$ the distinction seems pointless, but it was maintained in order to emphasize the relationships with second-order elliptic problems. These are also reflected by the role reversal of boundary conditions.

\subsection{Function spaces}

A Hilbert space framework provides the most powerful tools for the analysis of the linear variational problems (2.28) and (2.29) derived in the previous section. In the remainder of the article $\Omega \subset A\left(\mathbb{R}^{3}\right)$ stands for a bounded Lipschitz polyhedron with plane faces. More generally, in most contexts it could also be a curvilinear Lipschitz polyhedron in the parlance of Costabel and Dauge (1999). This will cover most geometric arrangements that occur in real world simulations. Throughout, $\mathbf{n} \in \boldsymbol{L}^{\infty}(\Gamma)$ will denote the exterior unit normal vector-field on $\Gamma$.

To obtain suitable Hilbert spaces for fields, we follow the usual procedure based on completions of spaces of smooth functions with respect to a norm induced by the total field energy. It is important to be aware that, for both the electric and magnetic field, the total field energy comprises contributions of magnetic and electric field energy. For instance, besides $E_{\mathrm{el}}(\mathbf{e})$ the total energy of an electric field solution of Maxwell's equations also involves the magnetic energy of curle. Appealing to the uniform positivity of the metric tensors, we arrive at the (equivalent) energy norm

$$
\|\mathbf{u}\|_{\boldsymbol{H}(\mathbf{c u r l} ; \Omega)}^{2}:=\|\mathbf{u}\|_{\boldsymbol{L}^{2}(\Omega)}^{2}+\|\operatorname{curl} \mathbf{u}\|_{\boldsymbol{L}^{2}(\Omega)}^{2} .
$$

The space obtained by completion of $\boldsymbol{C}^{\infty}(\Omega)$ with respect to $\|\cdot\|_{\boldsymbol{H}(\mathbf{c u r l} ; \Omega)}$ is customarily denoted by $\boldsymbol{H}(\mathbf{c u r l} ; \Omega) .{ }^{4}$ As the reader might guess, given a background of differential forms, $\boldsymbol{H}(\mathbf{c u r l} ; \Omega)$ is only one member of a larger family of Hilbert spaces. We could have introduced an 'energy norm' on smooth differential $l$-forms on $\Omega$ by

$$
\|\mathbf{u}\|_{\boldsymbol{H}(\boldsymbol{d}, \Omega)}^{2}:=\|\mathbf{u}\|_{\boldsymbol{L}^{2}(\Omega)}^{2}+\|\boldsymbol{d} \mathbf{u}\|_{\boldsymbol{L}^{2}(\Omega)}^{2},
$$

where the $\boldsymbol{L}^{2}(\Omega)$-norms are computed through some vector proxy. Note that the $\boldsymbol{L}^{2}(\Omega)$-norm of a vector proxy is, up to equivalence, independent of the Euclidean structure. Then $\boldsymbol{H}(\boldsymbol{d}, \Omega)$ can be defined as completion of $\mathcal{D F}^{l, \infty}(\Omega)$ with respect to $\|\cdot\|_{\boldsymbol{H}(\boldsymbol{d}, \Omega)}$. Recalling (2.15), this yields the familiar spaces $H^{1}(\Omega)$ and $\boldsymbol{H}(\operatorname{div} ; \Omega)$, corresponding to 0 -forms and 2-forms,

${ }^{4}$ Bold typeface is meant to distinguish vector-fields and spaces containing them. 
respectively. Important closed subspaces will be the kernels of the exterior derivatives, for which we write

$$
\boldsymbol{H}(\boldsymbol{d} 0, \Omega):=\{\mathbf{u} \in \boldsymbol{H}(\boldsymbol{d}, \Omega), \boldsymbol{d} \mathbf{u}=0\},
$$

in particular $\boldsymbol{H}(\operatorname{curl} 0 ; \Omega)$ and $\boldsymbol{H}(\operatorname{div} 0 ; \Omega)$. As it holds for smooth differential forms, the DeRham theorem (Theorem 2.1) can be extended to the function spaces. A particular case is given in the next lemma ( $c f$. Girault and Raviart (1986, Theorem 2.9), Amrouche, Bernardi, Dauge and Girault (1998, Proposition 3.14 and Proposition 3.18), Kress (1971)).

Lemma 2.2. There is a finite-dimensional cohomology space $\mathcal{H}^{1}(\Omega) \subset$ $\boldsymbol{H}(\boldsymbol{c u r l} 0 ; \Omega) \cap \boldsymbol{H}_{0}(\operatorname{div} 0 ; \Omega)$ of harmonic Neumann vector-fields whose dimension agrees with the first Betti number of $\Omega$, such that, for any $\mathbf{u} \in$ $\boldsymbol{H}(\operatorname{curl} 0 ; \Omega)$ with $\operatorname{curl} \mathbf{u}=0$, we find $\varphi \in H^{1}(\Omega)$ and $\mathbf{q} \in \mathcal{H}^{1}(\Omega)$ such that $\mathbf{u}=\operatorname{grad} \varphi+\mathbf{q}$.

Every $\mathbf{u} \in \boldsymbol{H}_{0}(\boldsymbol{c u r l} 0 ; \Omega)$ has a representation $\mathbf{u}=\operatorname{grad} \varphi+\mathbf{q}$, where $\varphi \in H_{0}^{1}(\Omega)$ and $\mathbf{q}$ is contained in a relative cohomology space $\mathcal{H}^{2}(\Omega) \subset$ $\boldsymbol{H}_{0}(\mathbf{c u r l} 0 ; \Omega) \cap \boldsymbol{H}(\operatorname{div} 0 ; \Omega)$ of harmonic Dirichlet vector-fields, $\operatorname{dim} \mathcal{H}^{2}(\Omega)=$ 2nd Betti number of $\Omega$.

As is proved in Girault and Raviart (1986, Theorem 2.40, Chapter I), an equivalent definition of the space $\boldsymbol{H}(\boldsymbol{\operatorname { c u r l }} ; \Omega)$ is

$$
\boldsymbol{H}(\operatorname{curl} ; \Omega):=\left\{\mathbf{u} \in \boldsymbol{L}^{2}(\Omega), \operatorname{curl} \mathbf{u} \in \boldsymbol{L}^{2}(\Omega)\right\},
$$

where curl has to be understood in the sense of distributions. Therefore, the variational equations (2.28) and (2.29) when considered over $\boldsymbol{H}(\mathbf{c u r l} ; \Omega)$ imply the topological laws (2.1) in the sense of distributions. Thus the ebased and $\mathbf{h}$-based variational formulations are indeed equivalent, and, for example, from (2.28) we can get $\mathbf{h}=\frac{1}{i \omega} \boldsymbol{\mu}^{-1}$ curle. As usual the following result is established ( $c f$. Lemmas 6 and 8 in Nédélec (1980)).

Lemma 2.3. If $\Omega \subset \mathbb{R}^{3}, \bar{\Omega}=\bar{\Omega}_{1} \cup \bar{\Omega}_{2}, \Omega_{1} \cap \Omega_{2}=\emptyset$, and $\mathbf{u}_{\mid \Omega_{1}} \in C^{\infty}\left(\bar{\Omega}_{1}\right)$, $\mathbf{u}_{\mid \Omega_{2}} \in \boldsymbol{C}^{\infty}\left(\bar{\Omega}_{2}\right)$, then

$$
\begin{array}{lll}
{\left[\gamma_{\mathbf{t}} \mathbf{u}\right]_{\partial \Omega_{1} \cap \partial \Omega_{2}}=0} & \Leftrightarrow & \mathbf{u} \in \boldsymbol{H}(\operatorname{curl} ; \Omega), \\
{\left[\gamma_{\mathbf{n}} \mathbf{u}\right]_{\partial \Omega_{1} \cap \partial \Omega_{2}}=0} & \Leftrightarrow & \mathbf{u} \in \boldsymbol{H}(\operatorname{div} ; \Omega) .
\end{array}
$$

We can also perform the completion of the space $\boldsymbol{C}_{0}^{\infty}(\Omega)$ of smooth vectorfields with compact support in $\Omega$ with respect to the energy norm. This results in the space $\boldsymbol{H}_{0}(\operatorname{curl} ; \Omega)$, a closed subspace of $\boldsymbol{H}(\operatorname{curl} ; \Omega)$, which realizes the condition of vanishing trace on $\Gamma$. This becomes evident by looking at the integration by parts formula $\left(\mathbf{u}, \mathbf{v} \in C^{\infty}(\bar{\Omega})\right)$

$$
\int_{\Omega} \mathbf{u} \cdot \operatorname{curl} \mathbf{v}-\operatorname{curl} \mathbf{u} \cdot \mathbf{v} \mathrm{d} \mathbf{x}=\int_{\Gamma}\left(\gamma_{\mathbf{t}} \mathbf{u} \times \mathbf{n}\right) \cdot \mathbf{v}_{\mid \Gamma} \mathrm{d} S .
$$


Appealing to a trace theorem for $\boldsymbol{H}^{1}(\Omega)$ (Grisvard 1985, Theorem 1.5.1.1), this confirms that the tangential trace $\gamma_{\mathbf{t}}: \boldsymbol{C}^{\infty}(\bar{\Omega}) \mapsto \boldsymbol{L}^{\infty}(\Gamma)$ is continuous as a mapping $\boldsymbol{H}(\operatorname{curl} ; \Omega) \mapsto \boldsymbol{H}^{-\frac{1}{2}}(\Gamma)$. Consequently, it can be extended to $\boldsymbol{H}(\mathbf{c u r l} ; \Omega)$. Since, obviously, $\gamma_{\mathbf{t}} \mathbf{u}=0$ for all $\mathbf{u} \in \boldsymbol{C}_{0}^{\infty}(\Omega)$, we get the alternative characterization

$$
\boldsymbol{H}_{0}(\operatorname{curl} ; \Omega):=\left\{\mathbf{u} \in \boldsymbol{H}(\operatorname{curl} ; \Omega), \gamma_{\mathbf{t}} \mathbf{u}=0\right\} .
$$

In many respects the space $\boldsymbol{H}(\mathbf{c u r l} ; \Omega)$ is rather unwieldy, in contrast to the classical Sobolev space $H^{1}(\Omega)$. Thus, the next lemma is instrumental in establishing key properties of $\boldsymbol{H}(\mathbf{c u r l} ; \Omega)$.

Lemma 2.4. (Regular decomposition lemma) There are continuous maps R : $\boldsymbol{H}(\operatorname{curl} ; \Omega) \mapsto \boldsymbol{H}^{1}(\Omega) \cap \boldsymbol{H}(\operatorname{div} 0 ; \Omega), \mathrm{N}: \boldsymbol{H}(\operatorname{curl} ; \Omega) \mapsto H^{1}(\Omega)$, $\mathrm{N}_{\mathcal{H}}: \boldsymbol{H}(\operatorname{curl} ; \Omega) \mapsto \mathcal{H}^{1}(\Omega)$ such that $\mathrm{R}+\operatorname{grad} \circ \mathrm{N}+\mathrm{N}_{\mathcal{H}}=\mathrm{Id}$ on $\boldsymbol{H}(\operatorname{curl} ; \Omega)$ and $\mathrm{R}_{\mid \boldsymbol{H}(\operatorname{curl} 0 ; \Omega)}=0$.

In addition, there are continuous maps $\mathrm{R}_{0}: \boldsymbol{H}_{0}(\operatorname{curl} ; \Omega) \mapsto \boldsymbol{H}^{1}(\Omega), \mathrm{N}_{0}$ : $\boldsymbol{H}_{0}(\operatorname{curl} ; \Omega) \mapsto H_{0}^{1}(\Omega)$ such that $\mathrm{R}_{0}+\operatorname{grad} \circ \mathrm{N}_{0}=\operatorname{Id}$ on $\boldsymbol{H}_{0}(\operatorname{curl} ; \Omega)$.

The proof will make use of the existence of regular vector potentials.

Lemma 2.5. (Existence of regular vector potentials) For every $r \geq$ 0 there is a continuous mapping $L: H\left(\operatorname{div} 0 ; \mathbb{R}^{3}\right) \cap \boldsymbol{H}^{r}\left(\mathbb{R}^{3}\right) \mapsto \boldsymbol{H}_{\mathrm{loc}}^{1+r}\left(\mathbb{R}^{3}\right)$ such that curl $L \mathbf{v}=\mathbf{v}$ and $\operatorname{div} L \mathbf{v}=0$.

The proof boils down to elementary calculations done with the Fourier transforms of the functions. It is given as part of the proof of Lemma 3.5 in Amrouche et al. (1998).

Proof of Lemma 2.4. For $\mathbf{u} \in \boldsymbol{H}(\mathbf{c u r l} ; \Omega)$ its rotation curl u belongs to $\boldsymbol{H}(\operatorname{div} 0 ; \Omega)$. Solving a Neumann problem for $\Delta$ outside $\Omega$, a divergencefree extension $\mathbf{v} \in \boldsymbol{H}\left(\operatorname{div} 0 ; \mathbb{R}^{3}\right)$ of $\mathbf{c u r l} \mathbf{u}$ can be found. Setting $\mathrm{Ru}:=\mathbf{L v}$, we find that $\operatorname{curl}(\mathbf{u}-\mathbf{R u})=0$ in $\Omega$ due to the properties of $L$. Applying Lemma 2.2 for $r=0$ finishes the first part of the proof.

The second part follows the proof of Proposition 5.1 in Bonnet-BenDhia, Hazard and Lohrengel (1999). A $\mathbf{u} \in \boldsymbol{H}_{0}(\mathbf{c u r l} ; \Omega)$ can be extended by zero to $\widetilde{\mathbf{u}} \in \boldsymbol{H}\left(\mathbf{c u r l} ; \mathbb{R}^{3}\right)$. Then curl $\widetilde{\mathbf{u}}$ belongs to the domain of $L$ for $r=0$. With $\boldsymbol{\Psi}:=\mathrm{L} \operatorname{curl} \widetilde{\mathbf{u}}$ we find that $\widetilde{\mathbf{u}}-\boldsymbol{\Psi}$ is curl-free. Thus there is a scalar potential $\psi \in H_{\text {loc }}^{1}\left(\mathbb{R}^{3}\right)$ with $\widetilde{\mathbf{u}}-\boldsymbol{\Psi}=\operatorname{grad} \psi$. As $\widetilde{\mathbf{u}}=0$ outside $\Omega, \psi \in H_{\text {loc }}^{2}\left(\mathbb{R}^{3} \backslash \bar{\Omega}\right)$. Write $\phi \in H^{2}(\Omega)$ for the Sobolev extension of $\psi_{\mid \mathbb{R}^{3} \backslash \bar{\Omega}}$ into the interior of $\Omega$. Then

$$
\mathbf{u}=(\Psi+\operatorname{grad} \phi)+\operatorname{grad}(\psi-\phi)
$$

is the desired decomposition and $\mathrm{R}_{0} \mathbf{u}:=\mathbf{\Psi}+\operatorname{grad} \phi, \mathrm{N}_{0} \mathbf{u}:=\psi-\phi$ defines the associated operators. 
Lemma 2.6. (More regular decomposition lemma) We can decompose every $\mathbf{u} \in \boldsymbol{H}(\mathbf{c u r l} ; \Omega)$ for which $\operatorname{curl} \mathbf{u} \in \boldsymbol{H}^{1}(\Omega)$, into $\mathbf{u}=\boldsymbol{\Psi}+$ $\operatorname{grad} \varphi+\mathbf{h}, \boldsymbol{\Psi} \in \boldsymbol{H}^{2}(\Omega), \varphi \in H^{1}(\Omega), \mathbf{h} \in \mathcal{H}^{1}(\Omega)$. Moreover, $\|\boldsymbol{\Psi}\|_{\boldsymbol{H}^{2}(\Omega)} \leq$ $C\|\operatorname{curl} \mathbf{u}\|_{\boldsymbol{H}^{1}(\Omega)}$ for some $C=C(\Omega)>0$ independent of $\mathbf{u}$.

Proof. The proof follows that of Corollary 3.3 in Girault and Raviart (1986) and, for the sake of simplicity, only tackles the case of a connected boundary $\partial \Omega$. Let $\mathcal{O}$ stand for a large ball containing $\bar{\Omega}$. Then the complement $\Omega^{\prime}:=\mathcal{O} \backslash \bar{\Omega}$ is a connected bounded Lipschitz domain. We exploit the important finding that grad : $L^{2}\left(\Omega^{\prime}\right) / \mathbb{R} \mapsto \boldsymbol{H}^{-1}\left(\Omega^{\prime}\right)$ is injective with closed range (Girault and Raviart 1986, Corollary 2.1). Thus, its $\boldsymbol{L}^{2}\left(\Omega^{\prime}\right)$-adjoint, $\operatorname{div}: \boldsymbol{H}_{0}^{1}\left(\Omega^{\prime}\right) \mapsto L_{\bullet}^{2}\left(\Omega^{\prime}\right)$ is surjective, where $L_{\bullet}^{2}\left(\Omega^{\prime}\right)$ contains all functions in $L^{2}\left(\Omega^{\prime}\right)$ with vanishing mean.

Extend curlu to $\mathbf{w} \in \boldsymbol{H}_{0}^{1}(\mathcal{O})$. Since $\int_{\partial \Omega} \operatorname{curl} \mathbf{u} \cdot \mathbf{n} \mathrm{d} S=0$, Gauss's theorem teaches that the mean of $\operatorname{div} \mathbf{w} \in L^{2}\left(\Omega^{\prime}\right)$ vanishes on $\Omega^{\prime}$. According to the above considerations, we can find $\mathbf{z} \in \boldsymbol{H}_{0}^{1}\left(\Omega^{\prime}\right)$ such that $\operatorname{div} \mathbf{z}=\operatorname{div} \mathbf{w}$. This means that

$$
\mathbf{v}:= \begin{cases}0, & \text { in } \mathbb{R}^{3} \backslash \overline{\mathcal{O}}, \\ \mathbf{w}-\mathbf{z}, & \text { in } \Omega^{\prime}, \\ \operatorname{curl} \mathbf{u}, & \text { in } \Omega\end{cases}
$$

belongs to $\boldsymbol{H}\left(\operatorname{div} 0 ; \mathbb{R}^{3}\right) \cap \boldsymbol{H}^{1}\left(\mathbb{R}^{3}\right)$. Using Lemma 2.5 for $r=1$ the proof can be completed in the same fashion as that of Lemma 2.4.

Remark 4. In one respect the completion procedure goes too far. Of course, it takes us beyond continuous functions, but we may wonder whether $\boldsymbol{H}(\mathbf{c u r l} ; \Omega)$ supplies valid integral 1-forms in the sense that the evaluation of integrals along piecewise smooth curves is a well-defined, that is, continuous, functional on $\boldsymbol{H}(\mathbf{c u r l} ; \Omega)$. Unfortunately, the answer is negative, and counterexamples are supplied by gradients of unbounded functions in $H^{1}(\Omega)$ and paths running through their singularity. This shortcoming of $\boldsymbol{H}(\mathbf{c u r l} ; \Omega)$ will be the source of considerable complications in the analysis of numerical methods ( $c f$. Section 3.6, in particular Lemma 3.13).

\section{Bibliographical notes}

The theory of the spaces $\boldsymbol{H}(\mathbf{c u r l} ; \Omega)$ and $\boldsymbol{H}(\operatorname{div} ; \Omega)$ is developed, for instance, in Girault and Raviart (1986, Chapter 1), Dautray and Lions (1990, Vol. 3, Chapter IX, Section 1), Fernandes and Gilardi (1997), and Amrouche et al. (1998). General information on Sobolev spaces can be found in Adams (1975) and Maz'ya (1985). Those for differential forms are discussed in Iwaniec (1999, Chapter 3) and Schwarz (1995, Chapter 1). The regular decomposition lemma first appeared in Birman and Solomyak (1990) and was implicitly used in the proof of Theorem 2 in Costabel (1990). More 
sophisticated decomposition theorems can be found in Bonnet-BenDhia et al. (1999) and Costabel, Dauge and Nicaise (1999).

\section{Discrete differential forms}

The perspective of differential forms offers an invaluable guideline for the discretization of Maxwell's equations. It will turn out that discrete differential forms, finite elements for differential forms, are the right tools for this task. Their roots in discrete cohomology theory on cellular complexes guarantee that essential structural properties of the topological laws of electromagnetism are preserved in a discrete setting.

\subsection{Cochains}

It is the very nature of discrete entities that they can be described by a finite amount of information. Recalling the notion of integral forms from Definition 1 , it is natural to demand that a discrete integral $l$-form on the domain $\Omega \subset A\left(\mathbb{R}^{3}\right)$ is already determined by fixing the numbers it assigns to a finite number of compact, oriented, piecewise smooth $l$-dimensional sub-manifolds of $\Omega$. In order to obtain a meaningful exterior derivative for discrete forms, these sets of special sub-manifolds must support boundary operators $\partial$. Therefore, one is led to consider triangulations of $\Omega$ as the natural device to construct appropriate finite sets of sub-manifolds.

Definition 3. A triangulation or mesh $\Omega_{h}$ of $\Omega \subset A\left(\mathbb{R}^{3}\right)$ is a finite collection of oriented cells (set $\mathcal{S}_{3}\left(\Omega_{h}\right)$ ), faces (set $\mathcal{S}_{2}\left(\Omega_{h}\right)$ ), edges (set $\mathcal{S}_{1}\left(\Omega_{h}\right)$ ), and vertices (set $\mathcal{S}_{0}\left(\Omega_{h}\right)$ ) such that:

- all cells are open subsets of $A\left(\mathbb{R}^{3}\right)$, homeomorphic to the unit ball;

- all cells, faces, edges, and vertices form a partition of $\bar{\Omega}$;

- the boundary of each cell is the union of closed faces, the boundary of each face the union of closed edges, and the boundary of each edge consists of vertices;

- each vertex, edge, and face is contained in the boundary of an edge, face, or cell, respectively.

The elements of $\mathcal{S}_{l}\left(\Omega_{h}\right)$ are called l-facets.

These triangulations are special cases of CW-complexes considered in discrete algebraic topology Lundell and Weingram (1969) and Fritsch and Piccini (1990). When augmented by orientation, all finite element meshes without hanging nodes will qualify as valid triangulations in the sense of Definition 3.

It remains to settle the issue of orientation. Let us first consider tetrahedral meshes ( $c f$. Bossavit (1998a, Section 5.2.1)). In this case any oriented 
$l$-facet is described by an $(l+1)$-tuple of vertices, whose order implies an (internal) orientation of the facet. The orientation of an $l$-facet induces an orientation of the $(l-1)$-facets contained in its boundary. For a tetrahedron $T=\left(\mathbf{a}_{0}, \mathbf{a}_{1}, \mathbf{a}_{2}, \mathbf{a}_{3}\right) \in \mathcal{S}_{3}\left(\Omega_{h}\right)$, the boundary faces carrying the induced orientation are given by

$$
\partial T \Rightarrow\left\{\left(\mathbf{a}_{2}, \mathbf{a}_{1}, \mathbf{a}_{0}\right),\left(\mathbf{a}_{0}, \mathbf{a}_{1}, \mathbf{a}_{3}\right),\left(\mathbf{a}_{3}, \mathbf{a}_{2}, \mathbf{a}_{0}\right),\left(\mathbf{a}_{1}, \mathbf{a}_{2}, \mathbf{a}_{3}\right)\right\} .
$$

The edges of an oriented face $F=\left(\mathbf{a}_{0}, \mathbf{a}_{1}, \mathbf{a}_{2}\right)$ turn out to be

$$
\partial F \quad \Rightarrow \quad\left\{\left(\mathbf{a}_{0}, \mathbf{a}_{1}\right),\left(\mathbf{a}_{2}, \mathbf{a}_{0}\right),\left(\mathbf{a}_{1}, \mathbf{a}_{2}\right)\right\} .
$$

The relative orientation of an $l$-1-facet $f$ in the boundary of an $l$-facet $F$ is $(-1)^{t}$, where $t$ is the number of transpositions of vertices it takes to convert the induced ordering of vertices into that fixing the interior orientation of $f$. The way to define orientation for facets of tetrahedral triangulations is neatly matched by data structures that can be used for their representation: if facet objects possess ordered lists referring to their vertices, an orientation is automatically implied.

Yet, for triangulations containing more general, say pyramidal and hexahedral, cells, it is awkward to rely on interior orientation alone. It is more convenient to rely on the external orientation of faces, which amounts to prescribing a crossing direction. Fixing an orientation of $A\left(\mathbb{R}^{3}\right)$, external and internal orientation are dual to each other (the 'corkscrew rule'). For edges, the usual internal orientation, their direction, is kept. Externally orienting the cells simply means declaring what is 'inside' and 'outside'. Then the induced external orientation of a face, contained in the boundary of a cell, is prescribed by crossing it from the interior to the exterior of the cell. Data structures representing this concept of orientation should supply references from faces to the adjacent cells.

We first look at precursors of discrete differential forms that already display an amazing wealth of structure.

Definition 4. An $l$-cochain $\vec{\omega}, 0 \leq l \leq 3,{ }^{5}$ on a triangulation $\Omega_{h}$ is a mapping $\mathcal{S}_{l}\left(\Omega_{h}\right) \mapsto \mathbf{C}$. The vector space of $l$-cochains on $\Omega_{h}$ will be denoted by $\mathcal{C}^{l}\left(\Omega_{h}\right)$. The value assigned by $\vec{\omega}$ to a collection of $l$-faces is computed as the sum of the values assigned to the individual $l$-faces.

This definition falls short of characterizing 'genuine cochains' studied in discrete cohomology theory on CW complexes. What is missing is the complementary concept of chains. However, Definition 4 suffices to convey the main ideas.

${ }^{5}$ We use the arrow notation to mark cochains, to emphasize that they can be described by 'coefficient vectors' in $\mathbb{C}^{N}$. No confusion should arise because bold typeface is used for vector-valued quantities. 


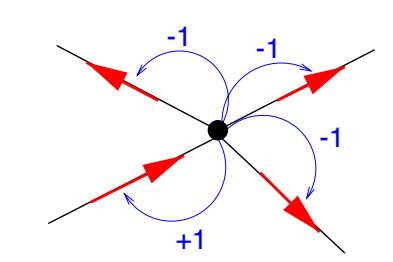

Topological gradient $\leftrightarrow \mathrm{D}^{0}$

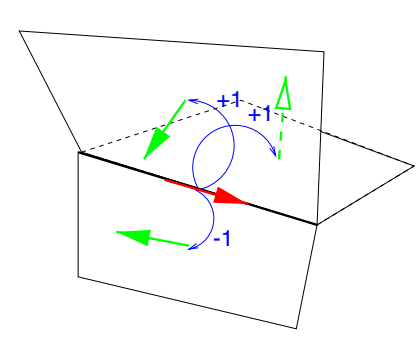

Topological rotation $\leftrightarrow \mathrm{D}^{1}$

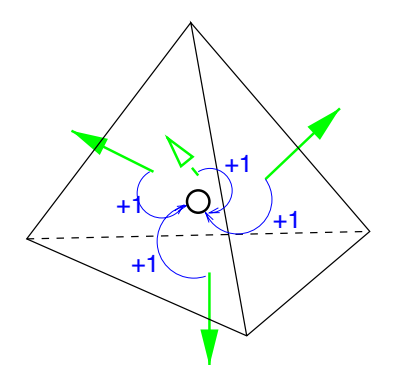

Topological divergence $\leftrightarrow \mathrm{D}^{2}$

Figure 3.1. Stencils for exterior derivative on cochains

It is clear that $\operatorname{dim} \mathcal{C}^{l}\left(\Omega_{h}\right)=\left|\mathcal{S}_{l}\left(\Omega_{h}\right)\right|^{6}$ and that, after ordering the $l$ faces of $\Omega_{h}$, we can identify $\mathcal{C}^{l}\left(\Omega_{h}\right)$ with $\mathbb{C}^{N_{l}}, N_{l}:=\left|\mathcal{S}_{l}\left(\Omega_{h}\right)\right|$. Such an identification will be taken for granted.

It is not difficult to devise cochain counterparts of all operators that we introduced for integral forms. First, a trace operator on $l$-cochains can be declared, in a natural way, as a mapping isolating the subset of coefficients belonging to $l$-facets of $\Omega_{h}$ contained in $\partial \Omega$. This gives a meaning to boundary conditions for cochains.

Thanks to the special properties of triangulations, an exterior derivative $\boldsymbol{d}_{h}: \mathcal{C}^{l}\left(\Omega_{h}\right) \mapsto \mathcal{C}^{l+1}\left(\Omega_{h}\right)$ can be defined by (2.7): for $\vec{\omega} \in \mathcal{C}^{l}\left(\Omega_{h}\right)$, its exterior derivative $\boldsymbol{d}_{h} \vec{\omega}$ assigns to each $F \in \mathcal{S}_{l+1}\left(\Omega_{h}\right)$ the sum of values $\vec{\omega}(f), f \in$ $\mathcal{S}_{l}\left(\Omega_{h}\right), f \subset \partial F$, weighted with the relative orientations (Gross and Kotiuga 2001, Section 2.1). This exterior derivative of cochains is a linear operator and the associated matrix $\mathrm{D}^{l} \in \mathbb{C}^{N_{l+1}, N_{l}}$ is the so-called incidence matrix of $l$-facets and $(l+1)$-facets of the triangulation, a sparse matrix with entries $\epsilon$ $\{-1,0,1\}$ determined by adjacency relations and relative orientations (Tonti 2001 , Section 4.2 ). The difference stencils representing $\mathrm{D}^{0}, \mathrm{D}^{1}$, and $\mathrm{D}^{2}$ are depicted in Figure 3.1.

I point out that the matrices $\mathrm{D}^{l}$ only depend on the topology of the triangulation. Also, $\mathrm{D}^{l+1} \mathrm{D}^{l}=0$ holds as well as a cochain counterpart of the exact sequence property.

Theorem 3.1. (DeRham theorem for cochains) There are finite-dimensional subspaces $\mathcal{H C}^{l}\left(\Omega_{h}\right) \subset \mathcal{C}^{l}\left(\Omega_{h}\right)$, $\operatorname{dim} \mathcal{H C}^{l}\left(\Omega_{h}\right)=l$ th Betti number of $\Omega$, such that, for $\vec{\omega} \in \mathcal{C}^{l}\left(\Omega_{h}\right)$, we have

$$
\mathrm{D}^{l} \vec{\omega}=0 \quad \Leftrightarrow \quad \exists \vec{\eta} \in \mathcal{C}^{l-1}\left(\Omega_{h}\right), \vec{\gamma} \in \mathcal{H C}^{l}\left(\Omega_{h}\right) \quad \text { satisfying } \vec{\omega}=\mathrm{D}^{l-1} \vec{\eta}+\vec{\gamma}
$$

Formally, we can state the topological laws of electromagnetism in the calculus of cochains as the following systems of linear equations:

$$
\mathrm{D}^{1} \overrightarrow{\mathbf{e}}=-i \omega \overrightarrow{\mathbf{b}}, \quad \mathrm{D}^{1} \overrightarrow{\mathbf{h}}=i \omega \overrightarrow{\mathbf{d}}+\overrightarrow{\mathbf{j}}
$$

\footnotetext{
${ }^{6}$ By $|X|$ we denote the cardinality of a finite set $X$.
} 
It can be connected to the topological Maxwell's equations (2.9) for integral forms through the so-called deRham maps

$$
\mathrm{I}_{l}: \mathcal{F}^{l}(\Omega) \mapsto \mathcal{C}^{l}\left(\Omega_{h}\right), \quad \mathrm{I}_{l}(\omega)(F)=\int_{F} \omega \quad \forall F \in \mathcal{S}_{l}\left(\Omega_{h}\right) .
$$

It is immediate from the definition of the exterior derivatives that

$$
\mathrm{D}^{l} \circ \mathrm{I}_{l}=\mathrm{I}_{l+1} \circ \boldsymbol{d}
$$

This has the important consequence that integral forms that are solutions of Maxwell's equations satisfy

$$
\mathrm{D}^{1} \mathbf{I}_{1} \mathbf{e}=-i \omega \mathbf{l}_{2} \mathbf{b}, \quad \mathrm{D}^{1} \mathbf{I}_{1} \mathbf{h}=\left.i \omega\right|_{2} \mathbf{d}+\mathrm{I}_{2} \mathbf{j} .
$$

Keep in mind that, for $l=1$, the DeRham map boils down to point evaluation. Also remember the notion of consistency error of finite difference methods. Thus, (3.4) means that the discrete topological laws (3.1) are consistent with their continuous counterparts (2.9).

Appealing to Theorem 3.1, we can also introduce cochain potentials $\overrightarrow{\mathbf{a}} \in$ $\mathcal{C}^{1}\left(\Omega_{h}\right), \vec{v} \in \mathcal{C}^{0}\left(\Omega_{h}\right)$, such that

$$
\overrightarrow{\mathbf{b}}=\mathrm{D}^{1} \overrightarrow{\mathbf{a}}, \quad \overrightarrow{\mathbf{e}}=-\mathrm{D}^{0} \vec{v}-i \omega \overrightarrow{\mathbf{a}} .
$$

\section{Bibliographical notes}

Cochains and their application to electromagnetism are discussed, for instance, in Bossavit (1998a, Section 5.3), Gross and Kotiuga (2001), Tarhasaari and Kettunen (2001) and Teixeira (2001, Section 2).

\subsection{Whitney forms}

It was the material laws that forced us to switch from integral forms to (local) differential forms. The material laws also fail to fit into the calculus of cochains. In order to accommodate them we have to use discrete differential forms, defined as true differential forms almost everywhere. They will allow the kind of local evaluations required to compute energies. However, we saw that cochains perfectly capture the topological laws. Therefore we opt for discrete differential forms that, sloppily speaking, extend cochains into the interior of cells and provide a model isomorphic to the calculus of cochains in terms of algebraic properties.

Formally, we seek bijective linear mappings $\mathrm{W}^{l}$, the so-called Whitney maps (Tarhasaari, Kettunen and Bossavit 1999), from $\mathcal{C}^{l}\left(\Omega_{h}\right)$ into a space of differential $l$-forms that are defined almost everywhere on $\Omega$ and make sense as integral forms on $\Omega$.

Definition 5. The range space $\mathrm{W}^{l}\left(\mathcal{C}^{l}\left(\Omega_{h}\right)\right), 0 \leq l \leq 3$ is called the space of Whitney $l$-forms $\mathcal{W}_{0}^{l}\left(\Omega_{h}\right)$ on the triangulation $\Omega_{h}$. 
Rather rigorous requirements have to be met by meaningful $\mathrm{W}^{l}$.

(1) The associated form has to be a true extension of the cochain, in the sense that

$$
\mathrm{I}_{l} \circ \mathrm{W}^{l}=\mathrm{Id} \quad \Leftrightarrow \quad \int_{F} \mathrm{~W}^{l} \vec{\omega}=\vec{\omega}(F) \quad \forall F \in \mathcal{S}_{l}\left(\Omega_{h}\right), \vec{\omega} \in \mathcal{C}^{l}\left(\Omega_{h}\right) .
$$

(2) The exterior derivatives of cochains and related Whitney forms must be linked by the commuting diagram

$$
\boldsymbol{d} \circ \mathrm{W}^{l}=\mathrm{W}^{l+1} \circ \mathrm{D}^{l} \quad \text { on } \mathcal{C}^{l}\left(\Omega_{h}\right) .
$$

(3) We demand strict locality: if all cochain coefficients of $\vec{\omega}$ associated with the $l$-facets belonging to a cell $T \in \mathcal{S}_{3}\left(\Omega_{h}\right)$ vanish, then $\mathrm{W}^{l} \vec{\omega}_{\mid T}=0$.

(4) The vector proxies of Whitney $l$-forms should be simple, that is, piecewise polynomial, on $\Omega_{h}$.

We first study the mappings $W^{l}$ for tetrahedral triangulations, the most important class of finite element meshes. We start with a 'local extension' on a single tetrahedron $T \in \mathcal{S}_{3}\left(\Omega_{h}\right)$ with vertices $\mathbf{a}_{0}, \mathbf{a}_{1}, \mathbf{a}_{2}, \mathbf{a}_{3}$. Cochain coefficients for all its facets are given. We take the cue from the case of 0 -forms, for which we know very well how to interpolate point values $\vec{\phi}\left(\mathbf{a}_{i}\right)$, that is, the coefficients of a 0 -cochain $\vec{\phi}$, prescribed in the vertices of $T$. Linear interpolation based on the barycentric coordinate functions $\lambda_{0}, \ldots, \lambda_{3}$ gives

$$
\phi(\mathbf{x})=\sum_{i=0}^{3} \vec{\phi}\left(\mathbf{a}_{i}\right) \lambda_{i}(\mathbf{x}) \quad \forall \mathbf{x} \in \bar{T} .
$$

In fact, this definition could be motivated by the identity $\mathbf{x}=\sum_{i=0}^{3} \mathbf{a}_{i} \lambda_{i}(\mathbf{x})$, that is, by representing any point in $T$ as a weighted combination of vertices. For 1-forms the role of vertices and points is played by edges and line segments. Any oriented line $(\mathbf{x}, \mathbf{y})$, with $\mathbf{x}, \mathbf{y} \in T, \mathbf{x}=\sum_{i} \lambda_{i}(\mathbf{x}) \mathbf{a}_{i}$, $\mathbf{y}=\sum_{i} \lambda_{i}(\mathbf{y}) \mathbf{a}_{i}$, can be represented as a 'weighted sum of edges of $T$ ':

$$
\begin{aligned}
(\mathbf{x}, \mathbf{y}) & =\{t \mathbf{x}+(1-t) \mathbf{y} ; 0 \leq t \leq 1\} \\
& =\left\{\sum_{i}\left(t \lambda_{i}(\mathbf{x})+(1-t) \lambda_{i}(\mathbf{y})\right) \mathbf{a}_{i} ; 0 \leq t \leq 1\right\} \\
& =\left\{\sum_{i}\left(t \sum_{j} \lambda_{j}(\mathbf{y}) \lambda_{i}(\mathbf{x})+(1-t) \sum_{j} \lambda_{j}(\mathbf{x}) \lambda_{i}(\mathbf{y})\right) \mathbf{a}_{i} ; 0 \leq t \leq 1\right\} \\
& =\left\{\sum_{i} \sum_{j} \lambda_{i}(\mathbf{x}) \lambda_{j}(\mathbf{y})\left(\left(t \mathbf{a}_{i}+(1-t) \mathbf{a}_{j}\right) ; 0 \leq t \leq 1\right\} .\right.
\end{aligned}
$$


Hence, taking into account orientation, we require that the interpolating differential 1-form $\mathrm{W}^{1} \vec{\omega}$ satisfies

$$
\int_{(\mathbf{x}, \mathbf{y})} \mathrm{W}_{\mid T}^{1} \vec{\omega}:=\sum_{i} \sum_{j} \lambda_{i}(\mathbf{x}) \lambda_{j}(\mathbf{y}) \vec{\omega}_{(i, j)}=\sum_{i<j}\left(\lambda_{i}(\mathbf{x}) \lambda_{j}(\mathbf{y})-\lambda_{i}(\mathbf{y}) \lambda_{j}(\mathbf{x})\right) \vec{\omega}_{(i, j)} .
$$

Here, $\vec{\omega}_{(i, j)}$ is the value that the 1-cochain $\vec{\omega}$ assigns to the oriented edge $\left(\mathbf{a}_{i}, \mathbf{a}_{j}\right)$. By construction, (3.7) is satisfied. So far, the formula fixes $\omega$ as an integral 1-form, but is it even a (local) differential 1-form? It is easy to see that a differential $l$-form on $T$ can be recovered from integral values through

$$
\omega(\mathbf{x})\left(\mathbf{v}_{1}, \ldots, \mathbf{v}_{l}\right):=l ! \lim _{t \rightarrow 0} \int_{\Sigma_{t}} \omega
$$

where $\Sigma_{t} \subset T$ is the $l$-simplex $\left(\mathbf{x}, \mathbf{x}+t \mathbf{v}_{1}, \ldots, \mathbf{x}+t \mathbf{v}_{l}\right), \mathbf{v}_{1}, \ldots, \mathbf{v}_{l} \in \mathbb{R}^{3}$. Using the local definition of the exterior derivative, we find

$$
\begin{aligned}
\left(\mathrm{W}_{\mid T}^{1} \vec{\omega}\right)(\mathbf{x})(\mathbf{v}) & =\lim _{t \rightarrow 0} \sum_{i<j}\left(\lambda_{i}(\mathbf{x}) \frac{\lambda_{j}(\mathbf{x}+t \mathbf{v})-\lambda_{j}(\mathbf{x})}{t}-\lambda_{j}(\mathbf{x}) \frac{\lambda_{i}(\mathbf{x}+t \mathbf{v})-\lambda_{i}(\mathbf{x})}{t}\right) \vec{\omega}_{(i, j)} \\
& =\sum_{i<j}\left(\lambda_{i}(\mathbf{x}) d \lambda_{j}(\mathbf{x})(\mathbf{v})-\lambda_{j}(\mathbf{x}) d \lambda_{i}(\mathbf{x})(\mathbf{v})\right) \vec{\omega}_{(i, j)} .
\end{aligned}
$$

Finally, the mapping $\mathrm{W}^{1}$ is built by combining the local interpolation operators $\mathrm{W}_{\mid T}^{1}$ for all cells $T \in \mathcal{S}_{3}\left(\Omega_{h}\right)$.

Observe that the values that the interpolant $W_{\mid T}^{1} \vec{\omega}$ assigns to line segments contained in a face of $T$ only depend on the cochain coefficients associated with edges of that face. A similar, even simpler statement can be made for edges: $\mathbf{W}_{\mid T}^{1} \vec{\omega}$ evaluated for parts of an edge $\left(\mathbf{a}_{i}, \mathbf{a}_{j}\right)$ only depends on $\vec{\omega}_{(i, j)}$. As a consequence, the above interpolation procedure applied to each tetrahedron of $\Omega_{h}$ will create locally defined smooth differential forms that satisfy the patch condition (2.20) for interelement faces. This confirms that $\mathrm{W}^{1}$ really maps into $\mathcal{F}^{1}(\Omega)$.

This procedure can even be generalized to the 'local interpolation formula' on an $n$-simplex $T \subset A\left(\mathbb{R}^{n}\right), n \in \mathbb{N}$ :

$$
\left(\mathbf{W}_{\mid T}^{l} \omega\right)(\mathbf{x})=\sum_{I} \sum_{j=0}^{l}(-1)^{j}(\underbrace{\lambda_{i_{j}} \bigwedge_{k=0, k \neq j}^{l} \boldsymbol{d} \lambda_{i_{k}}}_{\mathbf{b}_{I}}) \cdot \vec{\omega}_{I}
$$

where $I=\left(i_{0}, \ldots, i_{l}\right), 0 \leq l \leq n$, runs through all $(l+1)$-subsets of $\{0, \ldots, n\}$ and the ordering is induced by the orientation of the corresponding $l$-facet $\left(\left[\mathbf{a}_{i_{0}}, \ldots, \mathbf{a}_{i_{l}}\right)\right.$. The symbol $\vec{\omega}_{I}$ stands for the cochain coefficient associated with that facet. The $\mathbf{b}_{I}$ are called local basis forms. Their Euclidean vector 


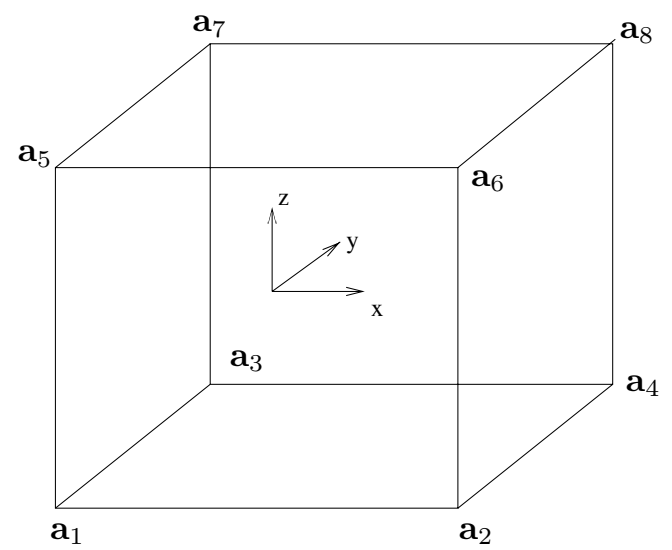

$$
\begin{aligned}
& \Upsilon_{1} \mathbf{b}_{(1,2)}=(1-y)(1-z) \cdot \mathbf{e}_{x} \\
& \Upsilon_{1} \mathbf{b}_{(3,4)}=y(1-z) \cdot \mathbf{e}_{x} \\
& \Upsilon_{1} \mathbf{b}_{(5,6)}=(1-y) z \cdot \mathbf{e}_{x} \\
& \Upsilon_{1} \mathbf{b}_{(7,8)}=y z \cdot \mathbf{e}_{x} \\
& \Upsilon_{1} \mathbf{b}_{(1,3)}=(1-x)(1-z) \cdot \mathbf{e}_{y} \\
& \Upsilon_{1} \mathbf{b}_{(2,4)}=x(1-z) \cdot \mathbf{e}_{y} \\
& \Upsilon_{1} \mathbf{b}_{(5,7)}=(1-x) z \cdot \mathbf{e}_{y} \\
& \Upsilon_{1} \mathbf{b}_{(6,8)}=x z \cdot \mathbf{e}_{y} \\
& \Upsilon_{1} \mathbf{b}_{(1,5)}=(1-y)(1-x) \cdot \mathbf{e}_{x} \\
& \Upsilon_{1} \mathbf{b}_{(2,6)}=(1-y) x \cdot \mathbf{e}_{x} \\
& \Upsilon_{1} \mathbf{b}_{(3,7)}=y(1-x) \cdot \mathbf{e}_{x} \\
& \Upsilon_{1} \mathbf{b}_{(4,8)}=y x \cdot \mathbf{e}_{x}
\end{aligned}
$$

Figure 3.2. Vector proxies of local basis forms for Whitney 1-forms on a brick, $\mathbf{e}_{x}=(1,0,0)^{T}, \mathbf{e}_{y}=(0,1,0)^{T}, \mathbf{e}_{z}=(0,0,1)^{T}$

proxies in three dimensions read

$$
\begin{aligned}
& \Upsilon_{0} \mathbf{b}_{(i)}=\lambda_{i}, \\
& \Upsilon_{1} \mathbf{b}_{(i, j)}=\lambda_{i} \operatorname{grad} \lambda_{j}-\lambda_{j} \operatorname{grad} \lambda_{i}, \\
& \Upsilon_{2} \mathbf{b}_{(i, j, k)}=\lambda_{i} \operatorname{grad} \lambda_{j} \times \operatorname{grad} \lambda_{k} \\
& +\lambda_{j} \operatorname{grad} \lambda_{k} \times \operatorname{grad} \lambda_{i}+\lambda_{k} \operatorname{grad} \lambda_{i} \times \operatorname{grad} \lambda_{j}, \\
& \Upsilon_{3} \mathbf{b}_{(0,1,2,3)}=1 / \operatorname{Vol}(T) \text {. }
\end{aligned}
$$

If $T$ is a brick, the extension of cochains can be done, too. Then the basis functions arise from a simple tensor-product-based construction. For $l=1$ they are given in Figure 3.2.

Finally we have come up with objects that fit the conventional notion of a finite element (Ciarlet 1978, Chapter 3) centring on the concept of local spaces and degrees of freedom (d.o.f.). In the case of Whitney forms, the local spaces $\mathcal{W}_{0}^{l}(T), T \in \mathcal{S}_{3}\left(\Omega_{h}\right)$ are supplied by the ranges of the local interpolation operators $\mathrm{W}_{\mid T}^{l}$. The integrals over $l$-facets are linear functionals on $\mathrm{W}_{\mid T}^{l}$ and serve as degrees of freedom. They are dual to the basis forms specified above. The expressions for Euclidean vector proxies are given in Table 3.1. By now it should have become clear why the Whitney 1-forms in three dimensions are usually called edge elements. This terms alludes to the 'location' of the degrees of freedom.

We stress that Whitney forms are affine equivalent in the following sense: If $\boldsymbol{\Phi}: \widehat{T} \mapsto T$ is the unique affine mapping between two tetrahedra, then

$$
\mathcal{W}_{0}^{l}(\widehat{T})=\boldsymbol{\Phi}^{*}\left(\mathcal{W}_{0}^{l}(T)\right)
$$


Table 3.1. Local spaces and degrees of freedom for Euclidean vector proxies of tetrahedral Whitney forms on a tetrahedron $T$ with vertices $\mathbf{a}_{0}, \ldots, \mathbf{a}_{3}$. Vertex indices have to be distinct

\begin{tabular}{ll}
\hline \multicolumn{1}{c}{ Local spaces } & \multicolumn{1}{c}{ Local d.o.f. } \\
\hline $\mathcal{W}_{0}^{0}(T)=\left\{\mathbf{x} \mapsto \mathbf{a} \cdot \mathbf{x}+\beta, \mathbf{a} \in \mathbb{R}^{3}, \beta \in \mathbb{R}\right\}$ & $u \mapsto u\left(\mathbf{a}_{i}\right)$ \\
$\mathcal{W}_{0}^{1}(T)=\left\{\mathbf{x} \mapsto \mathbf{a} \times \mathbf{x}+\mathbf{b}, \mathbf{a}, \mathbf{b} \in \mathbb{R}^{3}\right\}$ & $\mathbf{u} \mapsto \int_{\left(\mathbf{a}_{i}, \mathbf{a}_{j}\right)} \mathbf{u} \cdot \mathrm{d} \mathbf{s}$ \\
$\mathcal{W}_{0}^{2}(T)=\left\{\mathbf{x} \mapsto \alpha \mathbf{x}+\mathbf{b}, \alpha \in \mathbb{R}, \mathbf{b} \in \mathbb{R}^{3}\right\}$ & $\mathbf{u} \mapsto \int_{\left(\mathbf{a}_{i}, \mathbf{a}_{j}, \mathbf{a}_{k}\right)} \mathbf{u} \cdot \mathbf{n} \mathrm{d} S$ \\
$\mathcal{W}_{0}^{3}(T)=\{\mathbf{x} \mapsto \alpha, \alpha \in \mathbb{R}\}$ & $u \mapsto \int_{T} u \mathrm{~d} \mathbf{x}$ \\
\hline
\end{tabular}

and, as is clear from (2.12), the degrees of freedom retain their value under any pullback. We can even use (3.10) to define the local spaces for any diffeomorphic image of a tetrahedron. Thus we get, for instance, discrete differential forms on cells with curved boundaries, that is, parametric versions of Whitney forms. We could also have obtained them by observing that the interpolation procedure works with any set of functions $\lambda_{0}, \ldots, \lambda_{3}$ such that $\lambda_{i}\left(\mathbf{a}_{i}\right)=1$ and $\lambda_{i}, i=0,1,2,3$, vanishes on the face opposite to $\mathbf{a}_{i}$.

Combining the local interpolations according to (3.9) for all cells, we get the mappings $\mathrm{W}^{l}$ for $0 \leq l \leq 3$. This procedure ensures locality. The vector proxies are linear functions/vector-fields in each tetrahedron, as is clear from Table 3.1. It is as obvious that constant differential forms are contained in the local spaces $\mathcal{W}_{0}^{l}(T)$. Slightly more technical effort confirms that (3.7) is satisfied. Since the patch condition holds, Lemma 2.3 teaches that Whitney $l$-forms, $l=1,2$, furnish conforming finite elements.

Theorem 3.2. We have $\mathcal{W}_{0}^{1}\left(\Omega_{h}\right) \subset \boldsymbol{H}(\operatorname{curl} ; \Omega)$ and $\mathcal{W}_{0}^{2}\left(\Omega_{h}\right) \subset \boldsymbol{H}(\operatorname{div} ; \Omega)$.

Combining the extension of cochain with the deRham mapping yields the projections

$$
\Pi^{l}:=\mathrm{W}^{l} \circ \mathrm{I}_{l}: \mathcal{F}^{l}(\Omega) \mapsto \mathcal{W}_{0}^{l}\left(\Omega_{h}\right) \subset \mathcal{F}^{l}(\Omega),
$$

called the local interpolation operators to hint at their connection with the usual interpolation operators for finite elements that are based on degrees of freedom. This connection is illustrated by

$$
\int_{F} \omega-\Pi^{l} \omega=0 \quad \forall \omega \in \mathcal{F}^{l}(\Omega), F \in \mathcal{S}_{l}\left(\Omega_{h}\right) .
$$

When researching properties of discrete differential forms, there is a useful guiding principle supported by the fact that conventional Lagrangian finite elements are discrete 0-forms: 
Techniques that can be successfully applied to $H^{1}(\Omega)$-conforming Lagrangian finite elements can usually be adapted for other discrete differential forms, as well. Results obtained for Lagrangian finite elements are often matched by analogous results for other discrete differential forms.

The affine equivalence expressed in (3.10) is just one example of a property bearing out this guideline.

Yet there are aspects of discrete differential forms with no correspondents in the theory of Lagrangian finite elements. They chiefly have to do with relationships of discrete differential forms of different degree: from the properties of $\mathrm{W}^{l}$ we can readily deduce the commuting diagram property

$$
\boldsymbol{d} \circ \Pi^{l}=\Pi^{l+1} \circ \boldsymbol{d} .
$$

Therefore Theorem 3.1 will instantly carry over to Whitney forms.

Corollary 3.3. (DeRham theorem for Whitney forms) For each $1 \leq$ $l \leq n$ there are discrete cohomology spaces $\mathcal{H}_{h}^{l}\left(\Omega_{h}\right) \subset \mathcal{W}_{0}^{l}\left(\Omega_{h}\right)$, whose dimension agrees with the $l$ th Betti number of $\Omega$, such that, for any $\omega_{h} \in$ $\mathcal{W}_{0}^{l}\left(\Omega_{h}\right)$, we have

$\boldsymbol{d} \omega_{h}=0 \quad \Leftrightarrow \quad \exists \eta_{h} \in \mathcal{W}_{0}^{l-1}\left(\Omega_{h}\right), \gamma_{h} \in \mathcal{H}_{h}^{l}\left(\Omega_{h}\right) \quad$ satisfying $\omega_{h}=\boldsymbol{d} \eta_{h}+\gamma_{h}$.

Provided that $\Omega$ is homeomorphic to a ball, we can summarize our findings in the commuting diagram

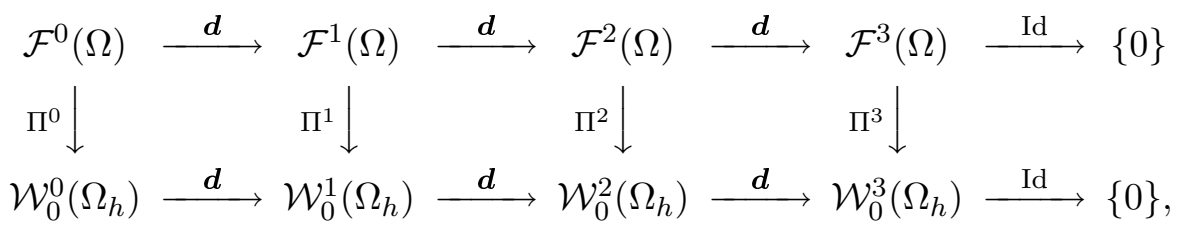

for which all the vertical sequences are exact.

Corollary 3.3 asserts the existence of discrete potentials in another space of Whitney forms. This is a really exceptional property. Imagine discrete 1-forms based on a continuous piecewise linear approximation of the components of vector proxies. If those have zero curl, there will exist a scalar potential on a domain with trivial topology. This scalar potential can be chosen to be piecewise polynomial of degree two and will feature $C^{1}$-continuity. Remember that $C^{1}$-finite elements require sophisticated constructions that have to rely on polynomials of degree greater than 2 . So we understand that the space of discrete potentials in this case cannot be a finite element space, because it will not possess a localized basis. Consequently, these discrete potentials cannot be handled efficiently in computations. Laconically, Whitney forms provide discrete potentials that are computationally available. 
Remark 5. Discrete cohomology spaces can fill the gap between irrotational vector-fields and gradients in the sense that

$$
\boldsymbol{H}(\operatorname{curl} 0 ; \Omega)=\operatorname{grad} H^{1}(\Omega) \oplus \mathcal{H}_{h}^{1}\left(\Omega_{h}\right) .
$$

Remark 6. One finds that the divergence of vector proxies of Whitney 1-forms vanishes locally on each tetrahedron. However, this just happens by accident and is irrelevant, because taking the (strong) divergence of the vector proxy of a discrete 1 -form is not quite meaningful.

Remark 7. In electrodynamics the Poynting vector is a 2-form $\mathbf{s}$ defined by $\mathbf{s}:=\mathbf{e} \wedge \mathbf{h}$. It describes the flux of electromagnetic energy. The definition of $\mathbf{s}$ hinges on a local interpretation of the forms. In other words, it takes us beyond cochain calculus, and Whitney forms are called for to give it a meaning in a discrete setting. Given discrete fields $\mathbf{e}_{h}$ and $\mathbf{h}_{h}$ in $\mathcal{W}_{0}^{1}\left(\Omega_{h}\right)$ we can easily compute $\mathbf{e}_{h} \wedge \mathbf{h}_{h}$ as a locally polynomial 2 -form. However, it is not a Whitney 2-form, because it features quadratic vector proxies.

Therefore, we may define the discrete Poynting vector as $\mathbf{s}_{h}=\Pi^{2}\left(\mathbf{e}_{h} \wedge \mathbf{h}_{h}\right)$. In order to compute the face fluxes of $\mathbf{s}_{h}$ efficiently, it is essential to recall that the pullback commutes with the $\wedge$-product. We learn that, for any face $F \in \mathcal{S}_{2}\left(\Omega_{h}\right)$ with edges $e_{1}, e_{2}, e_{3}$, we can obtain $\int_{S} \mathbf{s}_{h}$ as a weighted combination of the d.o.f. $\overrightarrow{\mathbf{e}}_{h}\left(e_{i}\right), \overrightarrow{\mathbf{h}}\left(e_{i}\right)$ with weights independent of the shape of $S$. Those can be computed for a single face, and it turns out that

$$
\overrightarrow{\mathbf{s}}_{h}(S)=\frac{1}{6}\left(\begin{array}{c} 
\pm \overrightarrow{\mathbf{h}}_{h}\left(e_{1}\right) \\
\pm \overrightarrow{\mathbf{h}}_{h}\left(e_{2}\right) \\
\pm \overrightarrow{\mathbf{h}}_{h}\left(e_{3}\right)
\end{array}\right){ }^{H}\left(\begin{array}{ccc}
0 & 1 & -1 \\
-1 & 0 & 1 \\
1 & -1 & 0
\end{array}\right)\left(\begin{array}{c} 
\pm \overrightarrow{\mathbf{e}}_{h}\left(e_{1}\right) \\
\pm \overrightarrow{\mathbf{e}}_{h}\left(e_{2}\right) \\
\pm \overrightarrow{\mathbf{e}}_{h}\left(e_{3}\right)
\end{array}\right)
$$

The signs have to be chosen in accordance with the relative orientations of the edges with respect to the face.

\section{Bibliographical notes}

Whitney forms were originally introduced by Whitney (1957) as a tool in algebraic topology. As such they have been used for a long time (Dodziuk 1976, Müller 1978). Their use as finite elements in computational electromagnetism was pioneered by A. Bossavit (1988a, 1988c, 1992, 1988b). A thorough discussion of elementary properties of Whitney forms is given in Bossavit (1998a, Chapter 5).

Independently of existing constructions in differential geometry, Whitney forms were rediscovered as mixed finite elements of lowest polynomial order (Raviart and Thomas 1977, Nédélec 1980). Their construction by interpolation is presented in Bossavit (2001, Section 7) and was used in Gradinaru and Hiptmair (1999) to construct Whitney forms on pyramids. 


\subsection{Discrete Hodge operators}

As $\boldsymbol{H}(\mathbf{c u r l} ; \Omega)$-conforming finite elements, Whitney 1-forms immediately lend themselves to a straightforward Galerkin discretization of the variational forms (2.28) and (2.29) of Maxwell's equations on bounded domains $\Omega \subset A\left(\mathbb{R}^{3}\right)$. Cochains have just served as a scaffolding to build the Whitney forms. This will be the favoured view in this article.

Yet, seen from a different angle, Whitney forms might be relegated to a mere tool to incorporate the material laws into the cochain framework. This point of view encompasses a much wider range of discretization schemes beyond Galerkin methods, in particular approaches known as finite volume/ finite integration schemes.

For the sake of simplicity we will consider only fields/cochains with zero trace on $\partial \Omega$. To begin with, we can expand the fields into a sum of basis forms, plug this into the variational forms $(2.24)-(2.27)$ of the material laws, also using Whitney forms as test fields. We get the following matrix equations for the expansion coefficients:

$$
\begin{aligned}
& (2.24) \Rightarrow \quad \widetilde{\mathrm{K}}_{2}^{1} \overrightarrow{\mathbf{d}}=\mathrm{M}_{\epsilon}^{1} \overrightarrow{\mathbf{e}}, \quad(2.26) \Rightarrow \quad \mathrm{K}_{2}^{1} \overrightarrow{\mathbf{b}}=\widetilde{\mathrm{M}}_{\mu}^{1} \overrightarrow{\mathbf{h}}, \\
& (2.25) \Rightarrow \quad \widetilde{\mathrm{K}}_{1}^{2} \overrightarrow{\mathbf{h}}=\mathrm{M}_{1 / \mu}^{2} \overrightarrow{\mathbf{b}}, \quad(2.27) \Rightarrow \quad \mathrm{K}_{1}^{2} \overrightarrow{\mathbf{e}}=\widetilde{\mathrm{M}}_{1 / \epsilon}^{2} \overrightarrow{\mathbf{d}} \text {. }
\end{aligned}
$$

The 'mass matrices' $\mathrm{M}_{\epsilon}^{1}, \mathrm{M}_{1 / \mu}^{2}, \widetilde{\mathrm{M}}_{\mu}^{1}, \widetilde{\mathrm{M}}_{1 / \epsilon}^{2}$, being related to energies, have to be real symmetric positive definite. The 'coupling matrices' $\widetilde{\mathrm{K}}_{2}^{1}, \widetilde{\mathrm{K}}_{1}^{2}, \mathrm{~K}_{2}^{1}$, and $\mathrm{K}_{1}^{2}$ introduce the exterior products of cochains. They are not regular, nor even square, in general. From the exterior product they will inherit the properties

$$
\mathrm{K}_{1}^{2}=\left(\widetilde{\mathrm{K}}_{2}^{1}\right)^{T}, \quad \widetilde{\mathrm{K}}_{1}^{2}=\left(\mathrm{K}_{2}^{1}\right)^{T},
$$

and, more importantly, the 'integration by parts formula'

$$
\left(\mathrm{D}^{1}\right)^{T} \widetilde{\mathrm{K}}_{1}^{2}=\widetilde{\mathrm{K}}_{2}^{1} \widetilde{\mathrm{D}}^{1} \quad \Leftrightarrow \quad\left(\widetilde{\mathrm{D}}^{1}\right)^{T} \mathrm{~K}_{1}^{2}=\mathrm{K}_{2}^{1} \mathrm{D}^{1} .
$$

As far as the topological laws are concerned, the fields $\mathbf{e}, \mathbf{b}$ and $\mathbf{h}, \mathbf{d}$ have nothing to do with each other. Recall that it was only the exterior derivative in the topological laws that forced us to use the same triangulation for cochains of different degree. Hence, $\mathbf{e}, \mathbf{b}$ (primal fields) and $\mathbf{h}, \mathbf{d}$ (dual fields) may well be discretized on unrelated triangulations $\Omega_{h}$ and $\widetilde{\Omega}_{h}$, called primal and dual hereafter. No problems are encountered in getting the material laws from their weak version by the Galerkin procedure. This observation explains the $\sim$ tag for matrices above. It distinguishes matrices acting on cochains defined on the triangulations for dual fields.

The above discrete material laws provide discrete Hodge operators for cochains. The idea is that a discretization of Maxwell's equations can be stated 
in terms of cochains assuming only discrete material laws with positive definite mass matrices and coupling matrices satisfying the algebraic properties (3.13) and (3.14).

Apart from the Galerkin approach there is another avenue to meaningful discrete Hodge operators. It starts with the consideration that the coupling matrices should be regular, reflecting the non-degeneracy of the exterior product. One may even demand that coupling matrices coincide with identity matrices. Then (3.14) involves

$$
\left(\mathrm{D}^{1}\right)^{T}=\widetilde{\mathrm{D}}^{1}, \quad\left(\widetilde{\mathrm{D}}^{1}\right)^{T}=\mathrm{D}^{1} .
$$

As the matrices $\mathrm{D}^{1}$ and $\widetilde{\mathrm{D}}^{1}$ are the edge-face incidence matrices of the primal and dual triangulations, we can achieve this by choosing $\widetilde{\Omega}_{h}$ as a triangulation dual to $\Omega_{h}$ (Gross and Kotiuga 2001, Section 5). This means there is a one-to-one correspondence of $\mathcal{S}_{l}\left(\Omega_{h}\right)$ and $\widetilde{\mathcal{T}}_{h}^{3-l}$. Prominent examples are geometrically dual triangulations, for which a $2 \mathrm{D}$ example is depicted in Figure 3.3. Here, the bijections between primal and dual facets are established by geometric intersection.

Special dual triangulations are orthogonal dual triangulations, for which primal edges are perpendicular to dual faces, as well as dual edges to primal faces Tonti (2001, Section 4), Marrone (2001, Section 3). In this case, provided we have to deal with material tensors that are multiples of the identity matrix, diagonal mass matrices can be chosen. To illustrate this consider the material law $\mathbf{d}=\boldsymbol{\epsilon} \mathbf{e}$ to be translated into $\overrightarrow{\mathbf{d}}=\mathrm{M}_{\epsilon}^{1} \overrightarrow{\mathbf{e}}$. Pick a single primal edge $e$ bearing a coefficient for $\overrightarrow{\mathbf{e}}$ and the associated dual face $\widetilde{F}$, for which we seek the coefficient for $\overrightarrow{\mathbf{d}}$. The situation is sketched

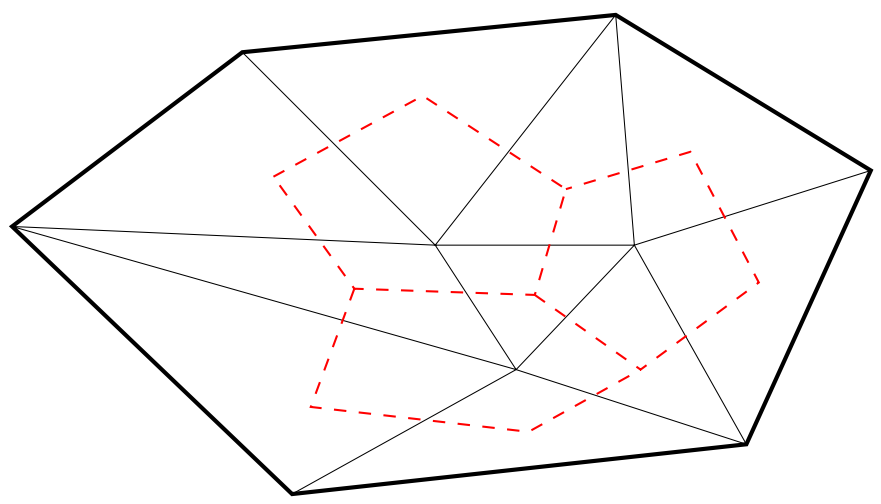

Figure 3.3. Triangulation (solid lines) and dual triangulation (dashed lines) in two dimensions 
in Figure 3.4. In terms of vector proxies we have $\mathbf{d}=\boldsymbol{\epsilon} \mathbf{e}$ and, therefore, because the face normal $\mathbf{n}$ runs parallel to $e$,

$$
\begin{aligned}
\overrightarrow{\mathbf{d}}(\widetilde{F}) & =\int_{\widetilde{F}} \mathbf{d} \cdot \mathbf{n} \mathrm{d} S \approx \operatorname{Vol}(\widetilde{F}) \mathbf{d}(\mathbf{p}) \cdot \mathbf{n}(\mathbf{p})=\operatorname{Vol}(\widetilde{F}) \boldsymbol{\epsilon}(\mathbf{p}) \mathbf{e}(\mathbf{p}) \cdot \mathbf{n} \\
& =\boldsymbol{\epsilon}(\mathbf{p}) \frac{\operatorname{Vol}(\widetilde{F})}{\operatorname{Vol}(e)} \mathbf{e}(\mathbf{p}) \cdot e \approx \boldsymbol{\epsilon}(\mathbf{p}) \frac{\operatorname{Vol}(\widetilde{F})}{\operatorname{Vol}(e)} \int_{e} \mathbf{e} \cdot \mathrm{d} \mathbf{s}=\boldsymbol{\epsilon}(\mathbf{p}) \frac{\operatorname{Vol}(\widetilde{F})}{\operatorname{Vol}(e)} \overrightarrow{\mathbf{e}}(e) .
\end{aligned}
$$

Thus, the entry of $\mathrm{M}_{\epsilon}^{1}$ associated with $e$ is equal to $\epsilon \operatorname{Vol}(\widetilde{F}) / \operatorname{Vol}(e)$. Yet the existence of orthogonal dual triangulations hinges on the Delaunay property of $\Omega_{h}$, which might be difficult to ensure for tetrahedral triangulations. If $\Omega_{h}$ consists of bricks, the same will be true of the dual grid and the construction is straightforward. The discrete Hodge operators obtained thus characterize the venerable Yee scheme (Yee 1966) and its more general version, the finite integration technique (Weiland 1996, Clemens and Weiland 2001).

Using the topological laws (3.1) for cochains, the discrete material laws, and the integration by parts formulas (3.14), we can perform the complete elimination of either the primal and dual quantities. Thus we arrive at the 'primal' discrete equations

$$
\left(\mathrm{D}^{1}\right)^{T} \mathrm{M}_{1 / \mu}^{2} \mathrm{D}^{1} \overrightarrow{\mathbf{e}}-\omega^{2} \mathrm{M}_{\epsilon}^{1} \overrightarrow{\mathbf{e}}=\widetilde{\mathrm{K}}_{2}^{1} \overrightarrow{\mathbf{j}}
$$

and their 'dual' versions

$$
\left(\widetilde{\mathrm{D}}^{2}\right)^{T} \mathrm{M}_{1 / \epsilon}^{2} \widetilde{\mathrm{D}}^{1} \overrightarrow{\mathbf{h}}-\omega^{2} \mathrm{M}_{\mu}^{1} \overrightarrow{\mathbf{h}}=\left(\widetilde{\mathrm{D}}^{2}\right)^{T} \mathrm{M}_{1 / \epsilon}^{2} \overrightarrow{\mathbf{j}}
$$

Surprisingly, the coupling matrices have largely disappeared, and we end up with equations that differ from the Galerkin equations only by a more general

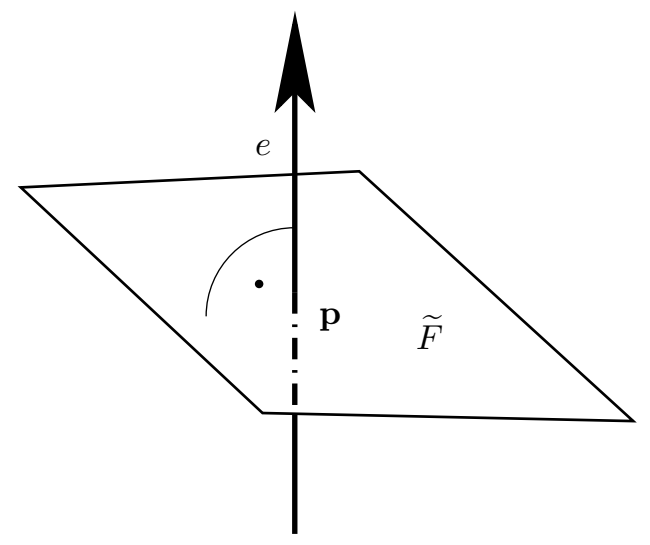

Figure 3.4. Primal edge and orthogonal dual face 
choice of mass matrices. The conclusion is that discretization schemes that can be put into the framework of discrete Hodge operators can be analysed as Galerkin schemes with perturbed mass matrices. This is why we mainly look at the Galerkin approach as the generic case.

\section{Bibliographical notes}

Recently, the theoretical foundations of discrete models for the laws of electromagnetism have seen a surge in interest (Bossavit and Kettunen 1999a, Bossavit and Kettunen 1999b, Tarhasaari et al. 1999, Teixeira and Chew 1999, Teixeira 2001, Tonti 2001, Tonti 1996, Mattiussi 2000). The rationale behind the concept of discrete Hodge operators is elucidated in Tarhasaari et al. (1999) and Mattiussi (2000). A comprehensive theory of discrete Hodge operators including convergence estimates has been developed in Hiptmair (2001b) and (2001a), extending ideas of Nicolaides and Wang (1998) and Nicolaides and Wu (1997). Many apparently different schemes, known as finite volume methods (Yee 1966), mimetic finite differences (Hyman and Shashkov 2001, 1999), cell methods (Marrone 2001), and finite integration techniques (Clemens and Weiland 2001, Weiland 1996, Schuhmann and Weiland 2001), emerge from particular realizations of discrete Hodge operators. We remind readers that the discrete formulation of electrodynamics, based on cochains and discrete Hodge operators, is only one example of a discrete 'lattice model', which plays an increasingly important role in physics (Dezin 1995, Mercat 2001).

\subsection{Higher-order Whitney forms}

The Whitney forms constructed in Section 3.2 are piecewise linear. Yet, for any $p \in \mathbb{N}_{0}$ we know $H^{1}(\Omega)$-conforming Lagrangian finite elements on tetrahedral and hexahedral triangulations, whose local spaces contain all multivariate polynomials of degree $\leq p$. Heeding the guideline of Section 3.2, this strongly suggests that, for all other members of the family of discrete differential forms, such higher-order schemes are available.

First, only simplicial meshes $\Omega_{h}$ are considered, but in dimension $n \in \mathbb{N}$. This generality is worthwhile, because we will make heavy use of induction and recursion with respect to dimension. Thus, in fact, it is easier to present the developments for dimension $n \in \mathbb{N}$ than to discuss the cases $n=2$ and $n=3$ separately.

Initially, we only consider a single generic simplex $T \subset A\left(\mathbb{R}^{n}\right)$ with vertices $\mathbf{a}_{0}, \ldots, \mathbf{a}_{n}$. Let $\lambda_{i}, i=0, \ldots, n$, denote the related barycentric coordinate functions in $T$.

The local spaces $\mathcal{W}_{p}^{l}(T)$ should contain polynomials. To state this, we introduce $\mathcal{P}_{p}(T)$ as the space of $n$-variate polynomials of total degree $\leq p$ 
on $T$. Then we define the space of polynomial differential $l$-forms on $T$, $0 \leq l \leq n$,

$$
\mathcal{D P}_{p}^{l}(T):=\left\{\omega=\sum_{I \subset\{1, \ldots, n\},|I|=l} \alpha_{I} \boldsymbol{d} \lambda_{I}, \alpha_{I} \in \mathcal{P}_{p}(T)\right\},
$$

where $I=\left\{i_{1}, \ldots, i_{l}\right\}, \boldsymbol{d} \lambda_{I}:=\boldsymbol{d} \lambda_{i_{1}} \wedge \cdots \wedge \boldsymbol{d} \lambda_{i_{l}}, i_{1}<i_{2}<\cdots<i_{l}$. Stated differently, a polynomial differential form possesses (component-wise) polynomials as vector proxies, regardless of the choice of basis.

The local spaces $\mathcal{W}_{p}^{l}(T)$ should contain all of $\mathcal{D P}_{p}^{l}(T)$, and should themselves be polynomial. This inspires the requirement

$$
\mathcal{D} \mathcal{P}_{p}^{l}(T) \subset \mathcal{W}_{p}^{l}(T) \subset \mathcal{D} \mathcal{P}_{p^{\prime}}^{l}(T), \quad \text { for some } p^{\prime} \geq p \geq 0 .
$$

We also argue that the exact sequence property of Whitney forms must remain true for higher-order discrete differential forms. A necessary condition is

$$
\left\{\omega \in \mathcal{W}_{p}^{l}(T), \boldsymbol{d} \omega=0\right\}=\boldsymbol{d} \mathcal{W}_{p}^{l-1}(T) \quad \forall 1 \leq l \leq n, p \in \mathbb{N}_{0} .
$$

The idea of the construction of the local spaces $\mathcal{W}_{p}^{l}(T)$ takes the cue from the $p$-version for $H^{1}(\Omega)$-conforming finite elements (Schwab 1998, Babuška and Suri 1994). There the usual approach is to associate with each $m$ facet $S \in \mathcal{S}_{m}(T)$ spaces of polynomials of degree $p_{S}$, whose traces on $\partial S$ vanish (Khoromskij and Melenk 2001, Section 3.1). These polynomials are extended into the interior of $T$ such that they have zero trace on any other $m$-facet of $T$. The span of the extended polynomials constitutes the desired local space. This simple procedure for 0 -forms is beset with tremendous technical difficulties for $l>0$. The culprits are the constraint (3.17), which is no longer immaterial for $l>0$, and the lack of a straightforward 'trace compliant' extension into $T$.

To begin with, suitable polynomial spaces $\mathcal{X}_{p}^{l}(S)$ associated with an $m$ facet $S \in \mathcal{S}_{m}(T)$ of $T, l \leq m \leq n$, have to be found. Analogously to the case of 0 -forms they must satisfy (for some $p^{\prime} \geq p$ )

$$
\mathcal{X}_{p}^{l}(S) \subset \mathcal{Z}_{p^{\prime}}^{l}(S):= \begin{cases}\left\{\omega \in \mathcal{D} \mathcal{P}_{p^{\prime}}^{l}(S), \mathbf{t}_{\partial S} \omega=0\right\}, & \text { for } l<m, \\ \left\{\omega \in \mathcal{D} \mathcal{P}_{p^{\prime}}^{m}(S), \int_{S} \omega=0\right\}, & \text { for } l=m .\end{cases}
$$

In addition they have to accommodate the restriction of (3.16) and (3.17) to $S$, that is, $\mathcal{Z}_{p}^{l}(S) \subset \mathcal{X}_{p}^{l}(S)$ and

$$
\left\{\omega \in \mathcal{X}_{p}^{l}(S), \boldsymbol{d} \omega=0\right\}=\boldsymbol{d} \mathcal{X}_{p}^{l-1}(S) \quad \forall 1 \leq l \leq m, p \in \mathbb{N}_{0} .
$$

As a next step, functions from the facet-based spaces $\mathcal{X}_{p}^{l}(S)$ have to be extended into the interior of $T$, without 'polluting' spaces on other facets of the same dimension. For polynomial 0-forms this can easily be achieved. 
If $S=\left(\mathbf{a}_{0}, \ldots, \mathbf{a}_{m}\right)$, every $\omega \in \mathcal{X}_{p}^{0}(S), p>m$, has a representation

$$
\omega=\alpha\left(\lambda_{0}, \ldots, \lambda_{m}\right) \lambda_{0} \cdots \lambda_{m},
$$

where $\alpha$ is a homogeneous polynomial of degree $p-(m+1)$. This representation instantly provides the desired formula for a polynomial extension of $\omega$ that is zero on all other $m$-facets of $T$. For $l>0$ the extension to the interior of $T$ will rely on a generalization of this procedure and uses a symmetric barycentric representation formula. Writing $\lambda_{I}:=\lambda_{i_{1}} \cdots \lambda_{i_{l}}$ for $I=\left\{i_{1}, \ldots, i_{l}\right\}$, it can be stated as follows.

Lemma 3.4. (Symmetric barycentric form) If $S=\left(\mathbf{a}_{s_{0}}, \ldots, \mathbf{a}_{s_{m}}\right)$, $\left\{s_{0}, \ldots, s_{m}\right\} \subset\{0, \ldots, n\}$, then any $\omega \in \mathcal{Z}_{p}^{l}(S), l \leq m, p>m-l$, can be written as

$$
\omega=\sum_{I \subset\left\{s_{0}, \ldots, s_{m}\right\},|I|=l} \alpha_{I}\left(\lambda_{s_{0}}, \ldots, \lambda_{s_{m}}\right) \lambda_{I^{\prime}} \boldsymbol{d} \lambda_{I}
$$

with $\alpha_{I}$ belonging to the space $\widetilde{\mathcal{P}}_{q}\left(\mathbb{R}^{m+1}\right)$ of $(m+1)$-variate homogeneous polynomials of degree $q, q:=p-(m+1-l)$, and $I \cup I^{\prime}=\left\{s_{0}, \ldots, s_{m}\right\}$, $I \cap I^{\prime}=\emptyset$.

Proof. Starting from a true basis representation of $\omega$ in terms of $\boldsymbol{d} \lambda_{I}$, $I \subset\left\{s_{1}, \ldots, s_{m}\right\}$, the zero trace conditions on sub-facets of $S$ give additional relationships between the coefficients. The symmetric barycentric representation formula emerges after tedious technical manipulations.

At first, this formula involves the restrictions of the barycentric coordinates to $S$, but it also makes sense for barycentric coordinates on $T$. In this reading it defines a barycentric extension $\mathrm{E}_{p, S}^{l} \omega$ of $\omega \in \mathcal{Z}_{p}^{l}(S)$ to $\mathcal{D P}_{p}^{l}(T)$. The representation according to Lemma 3.4 is by no means unique, nor is the barycentric extension, but all choices have the elementary property

$$
\omega \in \mathcal{Z}_{p}^{l}(S): \quad \mathbf{t}_{S^{\prime}}\left(\mathrm{E}_{p, S}^{l} \omega\right)=0 \quad \forall S^{\prime} \in \mathcal{S}_{m}(T) \backslash\{S\} .
$$

In plain English, the barycentric extension features zero trace on all other $m$-facets of $T$. Of course, any barycentric extension also preserves the polynomial degree of $\omega$.

The construction of facet-based spaces $\mathcal{X}_{p}^{l}(S)$ is possible thanks to the following key result.

Lemma 3.5. (Polynomial potentials with boundary conditions) For any $m$-facet $S \in \mathcal{S}_{m}(T), 1 \leq m \leq n$, we find, for all $m<l \leq n$,

$$
\mathcal{N}_{p}^{l}(S):=\left\{\omega \in \mathcal{Z}_{p}^{l}(S), \boldsymbol{d} \omega=0 \text { on } S\right\}=\boldsymbol{d} \mathcal{Z}_{p+1}^{l-1}(S) .
$$


Proof. We may choose a coordinate system with origin in vertex $\mathbf{a}_{0}$ of $T$. The crucial device is the Poincaré mapping $\mathfrak{K}^{l}: \mathcal{D} \mathcal{F}^{l, 0}(T) \mapsto \mathcal{D} \mathcal{F}^{l-1,1}(T)$, defined by

$$
\left(\mathfrak{K}^{l} \omega\right)(\mathbf{x})\left(\mathbf{v}_{1}, \ldots, \mathbf{v}_{l-1}\right):=\int_{0}^{1} t^{l-1} \omega(t \mathbf{x})\left(\mathbf{x}, \mathbf{v}_{1}, \ldots, \mathbf{v}_{l-1}\right) \mathrm{d} t,
$$

for all $\mathbf{v}_{1}, \ldots \mathbf{v}_{l-1} \in \mathbb{R}^{n}, \mathbf{x} \in T, \omega \in \mathcal{D} \mathcal{F}^{l, 0}(T)$. According to Cartan (1967), formula (2.13.2), it satisfies

$$
\boldsymbol{d}\left(\mathfrak{K}^{l} \omega\right)+\mathfrak{K}^{l+1}(\boldsymbol{d} \omega)=\omega .
$$

The remainder of the proof relies on induction with respect to $m$. For $m=1$, plain integration along the edge $\mathrm{S}$ of a polynomial 1-form $\omega \in \mathcal{Z}_{p}^{1}(S)$ with zero average will provide a 0 -form $\pi, \boldsymbol{d} \pi=\omega$, of polynomial degree $p+1$ vanishing in the endpoints of $S$.

For $m>1$ pick some $\omega \in \mathcal{Z}_{p}^{l}(S), \boldsymbol{d} \omega=0$, and set $\eta:=\mathfrak{K}^{l} \omega$. The identity (3.22) guarantees $\boldsymbol{d} \eta=\omega$, and by a simple inspection of (3.21) we see that $\eta \in \mathcal{D} \mathcal{P}_{p+1}^{l-1}(S)$. A closer scrutiny of (3.21) also shows that $\mathbf{t}_{F} \eta=0$ for all faces $F \in \mathcal{S}_{m-1}(S)$ that have $\mathbf{a}_{0}$ as vertex. A single $(m-1)$-facet $\widetilde{F}$ of $S$ remains, on which the trace of $\eta$ may not be zero. However, if $l<m$, then $\mathbf{t}_{\partial \widetilde{F}} \eta=0$ so that $\mathbf{t}_{\widetilde{F}} \eta \in \mathcal{Z}_{p+1}^{l-1}(\widetilde{F})$. To see $\mathbf{t}_{\widetilde{F}} \eta \in \mathcal{Z}_{p+1}^{l-1}(\widetilde{F})$ for $l=m$, observe that

$$
\int_{\widetilde{F}} \eta=\int_{\partial S} \eta=\int_{S} \boldsymbol{d} \eta=\int_{S} \omega=0
$$

by definition of $\mathcal{Z}_{p}^{m}(S)$. Thus, the induction hypothesis for $m-1$ applied to $\widetilde{F}$ gives $\widetilde{\tau} \in \mathcal{Z}_{p+2}^{l-2}(\widetilde{F})$ with $\boldsymbol{d} \widetilde{\tau}=\mathbf{t}_{\widetilde{F}} \eta$. A barycentric extension gives $\tau \in$ $\mathcal{D} \mathcal{P}_{p+2}^{l-2}(S)$, whose trace on all other $(m-1)$-facets except $\widetilde{F}$ vanishes. Then $\pi:=\eta-\boldsymbol{d} \tau$ yields the desired potential, because differentiation invariably reduces the polynomial degree by at least one.

A simple construction of $\mathcal{X}_{p}^{l}(S)$ is immediately on hand, based on the choice

$$
\mathcal{X}_{p}^{l}(S)=\mathcal{Z}_{p+1}^{l}(S), \quad \mathcal{X}_{p}^{l-1}(S)=\mathcal{Z}_{p+2}^{l-1}(S)
$$

Lemma 3.5 will make (3.19) hold, but we have to put up with non-uniform polynomial degrees in the exact sequence $\mathcal{X}_{p+m}^{0}(S) \stackrel{\boldsymbol{d}}{\mapsto} \mathcal{X}_{p+m-1}^{1}(S) \stackrel{\boldsymbol{d}}{\mapsto} \ldots \stackrel{\boldsymbol{d}}{\mapsto}$ $\mathcal{X}_{p}^{m}(S)$. However, usually the focus will be on discrete differential forms of a particular degree. Then (3.23) supplies perfect discrete differential forms of polynomial degree $p$. Using the barycentric extension procedure, this results in the so-called second family of discrete differential forms. Hardly surprisingly, the local spaces turn out to be $\check{\mathcal{W}}_{p}^{l}(T)=\mathcal{D} \mathcal{P}_{p+1}^{l}(T)$. Symbols related to this second family will be tagged with ${ }^{`}$, e.g., $\check{\mathcal{W}}_{p}^{l}\left(\Omega_{h}\right)$. 
Yet a true generalization of Whitney forms should avoid the increase in polynomial degree accompanying a decrease of $l$. Witness the case of Whitney forms, whose local spaces are contained in $\mathcal{D P}_{1}^{l}(T)$ for all $l$. Therefore we tighten the requirement (3.19) by demanding $p^{\prime}=p+1$, which leads to the first family of discrete differential form, which includes the Whitney forms as lowest-order representatives.

The key to the construction is the use of some direct decomposition

$$
\mathcal{Z}_{p}^{l}(S)=\mathcal{Y}_{p}^{l}(S) \oplus \mathcal{N}_{p}^{l}(S)
$$

We find that the choice

$$
\mathcal{X}_{p}^{l}(S):=\mathcal{Z}_{p}^{l}(S)+\mathcal{Y}_{p+1}^{l}(S)=\boldsymbol{d} \mathcal{Y}_{p+1}^{l-1}(S) \oplus \mathcal{Y}_{p+1}^{l}(S)
$$

meets all the requirements put forth for $\mathcal{X}_{p}^{l}(S)$. Despite the considerable freedom involved in choosing $\mathcal{X}_{p}^{l}(T)$, the dimensions of these spaces are already fixed.

Theorem 3.6. (Dimensions of $\mathcal{X}_{p}^{l}$ ) If the facet-associated spaces $\mathcal{X}_{p}^{l}(S)$ satisfy (3.19) and $\mathcal{Z}_{p}^{l}(S) \subset \mathcal{X}_{p}^{l}(S) \subset \mathcal{Z}_{p+1}^{l}(S)$, they fulfil $\operatorname{dim} \mathcal{X}_{p}^{l}(S)=$ $\left(\begin{array}{c}m \\ l\end{array}\right)\left(\begin{array}{c}p+l \\ m\end{array}\right)$ if $l<n$, and $\operatorname{dim} \mathcal{X}_{p}^{l}(S)=\left(\begin{array}{c}p+m \\ m\end{array}\right)-1$ if $l=m$. In particular, $\mathcal{X}_{p}^{l}(S)=\{0\}$ if $m>l+p$.

Proof. Compare the considerations in Section 3 of Hiptmair (2001c).

Now let us fix the barycentric extension operators $\mathrm{E}_{p, S}^{l}: \mathcal{X}_{p}^{l}(S) \mapsto \mathcal{D} \mathcal{P}_{p}^{l}(T)$, $0 \leq l \leq n, p \in \mathbb{N}, S \in \mathcal{S}_{m}(T), l \leq m \leq n$. Using these, define $\mathrm{F}_{p, S}^{l}: \mathcal{X}_{p}^{l}(S) \mapsto$ $\mathcal{D} \mathcal{P}_{p}^{l}(T)$ by

$$
\mathrm{F}_{p, S}^{l} \omega:=\mathrm{E}_{p+1, S}^{l} \omega^{\perp}+\boldsymbol{d} \mathrm{E}_{p+1, S}^{l-1} \eta, \quad \omega=\omega^{\perp}+\boldsymbol{d} \eta,
$$

where $\omega^{\perp} \in \mathcal{Y}_{p+1}^{l}(S)$ and $\eta \in \mathcal{Y}_{p+1}^{l-1}(S)$ are unique by (3.25). Now, we are in a position to build the local spaces as a direct sum of facet contributions

$$
\mathcal{W}_{p}^{l}(T):=\mathcal{W}_{0}^{l}(T) \oplus\left(\bigoplus_{m=l}^{n} \bigoplus_{S \in \mathcal{S}_{m}(T)} F_{p, S}^{l}\left(\mathcal{X}_{p}^{l}(S)\right)\right)
$$

The facet-based construction paves the way for introducing the global space $\mathcal{W}_{p}^{l}\left(\Omega_{h}\right)$ of discrete differential $l$-forms on a simplicial triangulation $\Omega_{h}$. It can be achieved by using extensions according to (3.26) from $\mathcal{X}_{p}^{l}(S)$ into adjacent simplices for all $S, S \in \mathcal{S}_{m}\left(\Omega_{h}\right), l \leq m \leq n$. This yields a space of $\Omega_{h}$-piecewise polynomial $l$-forms, for which the patch condition (2.20) is automatically satisfied, that is, $\mathcal{W}_{p}^{l}\left(\Omega_{h}\right) \in \mathcal{F}^{l}(\Omega)$. 
There is no reason why the same polynomial degree $p$ should be assigned to each $m$-facet $S$. Rather, we could use a vector $\mathbf{p}:=\left(p_{S}\right)_{S}, S \in \mathcal{S}_{m}\left(\Omega_{h}\right)$, $l \leq m \leq n$, of different polynomial degrees $p_{S} \in \overline{\mathbb{N}}_{0}$. The construction of the resulting space $\mathcal{W}_{\mathbf{p}}^{l}\left(\Omega_{h}\right) \subset \mathcal{F}^{l}(\Omega)$ is carried out as above. This leads to the so-called variable degree $p$-version for discrete differential forms. As in the case of Whitney forms, discrete potentials exist.

Theorem 3.7. (DeRham theorem for higher-order discrete forms) Using the discrete cohomology spaces $\mathcal{H}_{h}^{l}\left(\Omega_{h}\right)$ introduced in Corollary 3.3, for all $\omega_{h} \in \mathcal{W}_{\underline{\mathbf{p}}}^{l}\left(\Omega_{h}\right)$, we have

$$
\boldsymbol{d} \omega_{h}=0 \quad \Leftrightarrow \quad \exists \eta_{h} \in \mathcal{W}_{\underline{\mathbf{p}}}^{l-1}\left(\Omega_{h}\right), \gamma_{h} \in \mathcal{H}_{h}^{l}\left(\Omega_{h}\right) \quad \text { satisfying } \omega_{h}=\boldsymbol{d} \eta_{h}+\gamma_{h} \text {. }
$$

Proof. By the commuting diagram property (3.12) we know $\boldsymbol{d} \Pi^{l} \omega_{h}=0$, which, thanks to the discrete exact sequence property, implies the existence of $\eta_{h}^{0} \in \mathcal{W}_{0}^{l}\left(\Omega_{h}\right)$ with $\boldsymbol{d} \eta_{h}^{0}=\Pi^{l} \omega_{h}$.

For $\omega_{h}^{\prime}:=\left(\operatorname{Id}-\Pi^{l}\right) \omega_{h}$ we can find purely local discrete potentials by a recursive procedure. As first step pick any $S \in \mathcal{S}_{l}\left(\Omega_{h}\right)$ and note that both $\mathbf{t}_{S} \omega_{h}^{\prime} \in \mathcal{X}_{p_{S}}^{l}(S)$ and $\boldsymbol{d} \mathbf{t}_{S} \omega_{h}^{\prime}=0$. This means that $\mathbf{t}_{S} \omega_{h}=\boldsymbol{d} \eta_{S}$ for some $\eta_{S} \in$ $\mathcal{Y}_{p_{S}+1}^{l-1}(S)$. Adding the extensions according to $(3.26)$ of all $\eta_{S}$ provides $\eta_{l}$.

At the beginning of the second step we replace $\omega_{h}^{\prime} \leftarrow \omega_{h}^{\prime}-\boldsymbol{d} \eta_{l}$. Then consider $S \in \mathcal{S}_{l+1}\left(\Omega_{h}\right)$ and find a potential $\eta_{S} \in \mathcal{X}_{p_{S}}^{l-1}(S)$. The sum of the extended $\eta_{S}$ will be $\eta_{l+1}$. This $(l-1)$-form will have zero trace on all $l$-facets on $\Omega_{h}$.

Carrying on with $(l+2)$-facets and $\omega_{h}^{\prime} \leftarrow \omega_{h}^{\prime}-\boldsymbol{d} \eta_{l+1}$, we finally reach the $n$-facets and $\eta_{h}:=\sum_{k=l}^{n} \eta_{k}$ will provide the desired potential $\in \mathcal{W}_{\underline{\mathbf{p}}}^{l-1}\left(\Omega_{h}\right)$.

Remark 8. The discrete potentials of closed forms in the $p$-hierarchical surplus space $\left(\operatorname{Id}-\Pi^{l}\right) \mathcal{W}_{\mathbf{p}}^{l}\left(\Omega_{h}\right)$ are local in the sense that, if the support of the form is confined to the elements adjacent to some facet, the same will hold true for some discrete potential. The theorem also illustrates that, in terms of cohomology, the higher-order discrete differential forms do not convey the slightest additional information.

From (3.25) we can conclude that we have $\mathcal{Z}_{p+1}^{l}(S)=\mathcal{X}_{p}^{l}(S)+\boldsymbol{d} \mathcal{X}_{p+1}^{l-1}(S)$. This carries over to the global spaces and means that the second family of discrete differential forms can be obtained as

$$
\check{\mathcal{W}}_{p}^{l}\left(\Omega_{h}\right)=\mathcal{W}_{p}^{l}\left(\Omega_{h}\right)+\boldsymbol{d} \mathcal{W}_{p+1}^{l-1}\left(\Omega_{h}\right) .
$$

The spaces of the second family simply emerge from those of the first family by adding some closed forms of the next-higher polynomial degree. This implies $\boldsymbol{d} \check{\mathcal{W}}_{p}^{l}\left(\Omega_{h}\right)=\boldsymbol{d} \mathcal{W}_{p}^{l}\left(\Omega_{h}\right)$.

One issue still looms large, namely how to construct concrete basis functions (shape functions) for higher-order discrete differential forms. In general, one starts with building bases for $\mathcal{X}_{p}^{l}(S)$ on a generic $m$-facet 
$S, l \leq m \leq 3$, the reference facet. This can be done based on the symmetric barycentric representation from Lemma 3.4 (aided by some symbolic expression manipulation software). Decisions on the choice of the algebraic complement $\mathcal{Y}_{p}^{l}(S)$ and the special properties of the basis functions (orthogonality, hierarchical arrangement) translate into linear constraints on the coefficients of the homogeneous polynomials. Ultimately it will be the variational problem to be discretized that determines what are good sets of basis functions. The objective may be to achieve the best possible condition for element matrices. As no variational problem has been specified, no recommendations can be given at this stage.

Once basis functions for reference facets are available in symmetric barycentric representation, the same formulas can be used for all other facets. This is the gist of an affine invariant construction of discrete differential forms ( $c f$. Section 3.2).

We want to mention special bases of $\mathcal{X}_{p}^{l}(S)$, the $p$-hierarchical bases $\mathrm{HB}\left(\mathcal{X}_{p}^{l}(S)\right)$, for $p \geq 1$ distinguished by the properties

- $\operatorname{HB}\left(\mathcal{X}_{p}^{l}(S)\right)$ is a basis of $\mathcal{X}_{p}^{l}(S)$ for all $p$,

- $\operatorname{HB}\left(\mathcal{X}_{p}^{l}(S)\right)=\mathrm{HB}\left(\mathcal{X}_{p-1}^{l}(S)\right) \cup \widetilde{\mathrm{HB}}\left(\mathcal{X}_{p}^{l}(S)\right)$,

where $\widetilde{\mathrm{HB}}\left(\mathcal{X}_{p}^{l}(S)\right)$ is a suitable subset of $\mathcal{X}_{p}^{l}(S)$. This induces a partitioning

$$
\mathrm{HB}\left(\mathcal{X}_{p}^{l}(S)\right)=\mathrm{HB}\left(\mathcal{X}_{0}^{l}(S)\right) \cup \widetilde{\mathrm{HB}}\left(\mathcal{X}_{1}^{l}(S)\right) \cup \cdots \cup \widetilde{\mathrm{HB}}\left(\mathcal{X}_{p}^{l}(S)\right) \text {. }
$$

Note that $\left|\widetilde{\mathrm{HB}}\left(\mathcal{X}_{p}^{l}(S)\right)\right|:=\left(\begin{array}{c}k+l-1 \\ n-1\end{array}\right)$. We can even select basis functions reflecting the splitting (3.24). Thus we get a hierarchical basis of the form

$$
\widetilde{\mathrm{HB}}\left(\mathcal{X}_{p}^{l}(S)\right)=\left\{\beta_{p, 1}^{l}, \ldots, \beta_{p, M_{p}^{l}}^{l}, \boldsymbol{d} \beta_{1, p}^{l-1}, \ldots, \boldsymbol{d} \beta_{p, M_{p}^{l-1}}^{l-1}\right\},
$$

Table 3.2. Numbers of basis functions associated with sub-simplices for tetrahedral discrete differential 1- and 2-forms and uniform polynomial degree $p$

\begin{tabular}{lcccccccr}
\hline \hline$p$ & & 0 & 1 & 2 & 3 & 4 & 5 & 6 \\
\hline$l=1$ & edges & 1 & 2 & 3 & 4 & 5 & 6 & 7 \\
& faces & 0 & 2 & 6 & 12 & 20 & 30 & 42 \\
& cell & 0 & 0 & 3 & 12 & 30 & 60 & 105 \\
\hline \multirow{2}{*}{$l=2$} & faces & 1 & 3 & 6 & 10 & 15 & 21 & 28 \\
& cell & 0 & 3 & 12 & 30 & 60 & 105 & 168 \\
& & & & & & & & \\
\hline
\end{tabular}


where $M_{p}^{0}=\left(\begin{array}{c}k-1 \\ n-1\end{array}\right)$ and $M_{p}^{l}+M_{p}^{l-1}=\left(\begin{array}{c}m \\ l\end{array}\right)\left(\begin{array}{c}p+l-1 \\ m-1\end{array}\right)$. In words, we can find a $p$-hierarchical basis for which all closed discrete $l$-forms in $p$-hierarchical surpluses are linear combinations of closed basis forms. The $p$-hierarchical basis of $\mathcal{W}_{\mathbf{p}}^{l}\left(\Omega_{h}\right)$ is straightforward by extension and combination.

From (3.28) it is immediate that we get a basis for $\check{\mathcal{W}}_{p}^{l}\left(\Omega_{h}\right)$ for uniform polynomial degree $p \in \mathbb{N}_{0}$ by augmenting the basis of $\mathcal{W}_{p}^{l}\left(\Omega_{h}\right)$ by (linearly independent) exterior derivatives of basis functions of $\mathcal{W}_{p+1}^{l-1}\left(\Omega_{h}\right)$.

Returning to tetrahedral triangulations, that is, $n=3$, the numbers of basis functions associated with different facets are listed in Table 3.2. The numbers of extra non-closed basis functions needed to build a hierarchical basis according to (3.29) are recorded in Table 3.3. Note that all higher-order basis functions on $l$-facets are closed.

In this fashion we can find the hierarchical basis for first-order 1-forms of the first family. According to Table 3.2 we expect two basis functions associated with each edge, and two more with each face. Their vector proxies in symmetric barycentric representation read

$$
\left.\begin{array}{l}
\lambda_{i} \operatorname{grad} \lambda_{j}-\lambda_{j} \operatorname{grad} \lambda_{i} \\
\lambda_{i} \operatorname{grad} \lambda_{j}+\lambda_{j} \operatorname{grad} \lambda_{i}
\end{array}\right\} \quad \text { on edge }\left[\mathbf{a}_{i}, \mathbf{a}_{j}\right],
$$

The basis functions belonging to the edges constitute a basis for the lowestorder discrete 1-forms of the second family.

Table 3.3. Numbers $M_{p}^{l}$ for a tetrahedron (uniform polynomial degree)

\begin{tabular}{lcccccccc}
\hline \hline$p$ & & {$[0]$} & 1 & 2 & 3 & 4 & 5 & 6 \\
\hline$l=0$ & vertices & {$[1]$} & 0 & 0 & 0 & 0 & 0 & 0 \\
& edges & {$[0]$} & 1 & 1 & 1 & 1 & 1 & 1 \\
& faces & {$[0]$} & 0 & 1 & 2 & 3 & 4 & 5 \\
& cell & {$[0]$} & 0 & 0 & 1 & 3 & 6 & 10 \\
\hline$l=1$ & edges & {$[1]$} & 0 & 0 & 0 & 0 & 0 & 0 \\
& faces & {$[0]$} & 2 & 3 & 4 & 5 & 6 & 7 \\
& cell & {$[0]$} & 0 & 3 & 8 & 15 & 24 & 35 \\
\hline$l=2$ & faces & {$[1]$} & 0 & 0 & 0 & 0 & 0 & 0 \\
& cell & {$[0]$} & 3 & 6 & 10 & 15 & 21 & 28 \\
\hline
\end{tabular}


For hexahedral triangulations similar strategies lead to higher-order discrete differential forms. We skip the discussion of the details and just present the results. For the first family of discrete differential forms the local spaces on a generic brick $T$ read

$$
\begin{aligned}
& \mathcal{W}_{p}^{1}(T)=Q_{p, p+1, p+1}(T) \times Q_{p+1, p, p+1}(T) \times Q_{p+1, p+1, p}(T), \\
& \mathcal{W}_{p}^{2}(T)=Q_{p+1, p, p}(T) \times Q_{p, p+1, p}(T) \times Q_{p, p, p+1}(T) .
\end{aligned}
$$

Here $Q_{p_{1}, p_{2}, p_{3}}(T)$ denotes the space of 3-variate polynomials of degree $\leq p_{i}$ in the $i$ th variable, $i=1,2,3$. The number of basis functions associated with edges, faces and cells is given in Table 3.4.

As for the case of tetrahedra, a second family of discrete differential forms on hexahedral features complete spaces of 3 -variate polynomials of degree $\leq p+1$ in each independent variable as local spaces.

\section{Bibliographical notes}

Higher-order discrete 1-forms in two dimensions are known as $\boldsymbol{H}(\operatorname{div} ; \Omega)$ conforming Raviart-Thomas finite elements, and were introduced in Raviart and Thomas (1977). The first family of discrete 1- and 2-forms in 3D was first presented in Nédélec (1980), and the second family in Nédélec (1986). The second family for $\boldsymbol{H}(\operatorname{div} ; \Omega)$-conforming finite elements is also known as BDM-elements (Brezzi, Douglas and Marini 1985). A complete survey of various discrete $(n-1)$-forms, $n=2,3$, is given in Brezzi and Fortin (1991, Chapter 3). The treatment in this section was first adopted in Hiptmair $(1999 a, 2001 c)$ and is also sketched in Demkowicz (2001).

The choice of $p$-hierarchical higher-order shape functions is dealt with in Sun, Lee and Cendes (2001) for tetrahedra, and Monk (1994) and Ainsworth

Table 3.4. Numbers of basis functions associated with edges, faces and cells for hexahedral discrete differential 1 - and 2-forms of the first family and uniform polynomial degree $p$

\begin{tabular}{lccccrrrr}
\hline \hline$p$ & & 0 & 1 & 2 & 3 & 4 & 5 & 6 \\
\hline$l=1$ & edges & 1 & 2 & 3 & 4 & 5 & 6 & 7 \\
& faces & 0 & 4 & 12 & 24 & 40 & 60 & 84 \\
& cell & 0 & 6 & 36 & 108 & 240 & 450 & 756 \\
\hline \multirow{2}{*}{$l=2$} & faces & 1 & 4 & 9 & 16 & 25 & 36 & 49 \\
& cell & 0 & 12 & 54 & 144 & 300 & 540 & 882 \\
\hline
\end{tabular}


and Coyle $(2001 a, 2001 b)$ for the use of Legendre polynomials on hexahedra/quadrilaterals. Shape functions for the second family of discrete differential forms on tetrahedra with variable polynomial degree are given in Demkowicz (2001). This survey article also discusses criteria for choosing basis functions. Issues of implementation are addressed in Vardapetyan and Demkowicz (1999) and Rachowicz and Demkowicz (2002).

\subsection{Interpolation operators}

With the spaces $\mathcal{W}_{\mathbf{p}}^{l}\left(\Omega_{h}\right)$ being completely defined, we may consider analogues of the local interpolation operators $\Pi^{l}$, which satisfy a commuting diagram property according to (3.12). The reader should be aware that these interpolation operators are never needed in an implementation of a finite element code based on discrete differential forms. In this respect only the shape functions are relevant. The interpolation operators discussed in this section are merely theoretical tools. For the same reason as in the previous section, we take a look at the general situation in dimension $n \in \mathbb{N}$.

In the case of interpolation operators and local bases alike we find that their construction is canonical only for Whitney forms, whereas for higherorder discrete differential forms we have numerous sensible options. First, we examine moment-based interpolation operators for the first family of simplicial discrete differential forms. Following a classical idea in the field of finite elements, they are based on degrees of freedom (Ciarlet 1978, Section 2.3), that is, a set $\Theta_{\underline{\mathbf{p}}}^{l}\left(\Omega_{h}\right)$ of linear forms $\mathcal{W}_{\underline{\mathbf{p}}}^{l}\left(\Omega_{h}\right) \mapsto \mathbb{R}$, such that:

(i) $\Theta_{\underline{\mathbf{p}}}^{l}\left(\Omega_{h}\right)$ is a dual basis of $\mathcal{W}_{\underline{\mathbf{p}}}^{l}\left(\Omega_{h}\right)$ (unisolvence);

(ii) to each facet $S \in \mathcal{S}_{m}\left(\Omega_{h}\right), l \leq m \leq n$, we can associate a set $\Theta_{p_{S}}^{l}(S) \subset$ $\Theta_{\underline{\mathbf{p}}}^{l}\left(\Omega_{h}\right)$. Each $\kappa \in \Theta_{\underline{\mathbf{p}}}^{l}\left(\Omega_{h}\right)$ belongs to exactly one of these sets, and

$$
\kappa\left(\omega_{h}\right)=0 \quad \forall \kappa \in \Theta_{p_{R}}^{l}(R), R \in \mathcal{S}_{k}(S), l \leq k \leq m \quad \Leftrightarrow \quad \mathbf{t}_{S} \omega_{h}=0
$$

for every $\omega_{h} \in \mathcal{W}_{\underline{\mathbf{p}}}^{l}(m)$. Thus the degrees of freedom have to be local.

Locality suggests a facet-based approach. For each $S \in \mathcal{S}_{m}\left(\Omega_{h}\right), l \leq m \leq$ $n$, we find linear forms $\zeta_{i}: \mathcal{X}_{p_{S}}^{l}(S) \mapsto \mathbb{R}, i=1, \ldots, \operatorname{dim} \mathcal{X}_{p_{S}}^{l}(S)$, that form a dual basis for $\mathcal{X}_{p_{S}}^{l}(S)$. Then set $\zeta_{i}^{S}:=\zeta_{i} \circ \mathbf{t}_{S}$ and observe that

$$
\Theta_{\underline{\mathbf{p}}}^{l}\left(\Omega_{h}\right):=\left\{\zeta_{i}^{S}, S \in \mathcal{S}_{m}(S), l \leq m \leq n, i=1, \ldots, \operatorname{dim} \mathcal{X}_{p_{S}}^{l}(S)\right\}
$$

constitutes a set of degrees of freedom meeting both of the above requirements. Just note that $\left\{\zeta_{i}^{S}\right\}_{i}$ is simply the subset of $\Theta_{\mathbf{p}}^{l}\left(\Omega_{h}\right)$ associated with $S$. It remains to find degrees of freedom for all $\mathcal{X}_{p_{S}}^{l}(S)$. 
Theorem 3.8. For every $\omega \in \mathcal{X}_{p}^{l}(S), S \in \mathcal{S}_{m}\left(\Omega_{h}\right), l \leq m \leq n, p \geq m-l$, we have

$$
\int_{S} \omega \wedge \mu=0 \quad \forall \mu \in \mathcal{D P}_{p-m+l}^{m-l}(S) \Leftrightarrow \omega=0 .
$$

Proof. (See the proof of Lemma 10 in Hiptmair (1999a).) The proof employs (descending) induction with respect to $l$. For $l=m$ we saw that $\mathcal{X}_{p}^{m}(S)+\mathcal{D} \mathcal{P}_{0}^{m}(S)=\mathcal{D} \mathcal{P}_{p}^{m}(S)$ and the assertion is evident, because for both $\mathcal{D} \mathcal{P}_{p}^{m}(S)$ and $\mathcal{D} \mathcal{P}_{p}^{0}(S)$ the vector proxies belong to the same space $\mathcal{P}_{p}(S)$.

In the case $l<m$, use $\mathbf{t}_{\partial S} \omega=0$ and integration by parts to obtain

$$
\int_{S} d \omega \wedge \mu= \pm \int_{S} \omega \wedge \boldsymbol{d} \mu=0
$$

for all $\mu \in \mathcal{D P}_{p-m+l+1}^{m-(l+1)}(S)$. As $\boldsymbol{d} \omega \in \mathcal{X}_{p}^{l+1}(S)$, the induction hypothesis for $l+1$ involves $\boldsymbol{d} \omega=0$. By definition of $\mathcal{X}_{p}^{l}(S)$ in formula (3.25), we can conclude that $\omega \in \mathcal{D} \mathcal{P}_{p}^{l}(S)$. In particular, we can find a representation

$$
\omega=\sum_{I \subset\{1, \ldots, m\},|I|=l} \beta_{I}\left(\lambda_{1}, \ldots, \lambda_{m}\right) \lambda_{I^{\prime}} \boldsymbol{d} \lambda_{I},
$$

$I^{\prime} \cup I=\{1, \ldots, m\}, I \cap I^{\prime}=\emptyset, \beta_{I} \in \mathcal{P}_{p-(m-l)}\left(\mathbb{R}^{m}\right)$. Here $\lambda_{i}, i=1, \ldots, m$, are local barycentric coordinates of $S$. Pick $\mu=\lambda_{J} \boldsymbol{d} \lambda_{J}, J \subset\{1, \ldots, m\}$, $|J|=m-l$, and verify

$$
\int_{S} \omega \wedge \mu= \pm \int \beta_{J^{\prime}} \lambda_{J}^{2} \boldsymbol{d} \lambda_{1} \wedge \cdots \wedge \boldsymbol{d} \lambda_{m}
$$

As $\mu \in \mathcal{D P}_{m-l}^{m-l}(S)$ this has evaluate to zero, which immediately implies $\beta_{J^{\prime}}=0, J \cup J^{\prime}=\{1, \ldots, m\}$. By varying $J$, all $\beta_{I}$ in (3.30) are seen to vanish.

Using well-known formulas for dimensions of spaces of polynomials, it is immediate that, for $m>l$,

$$
\operatorname{dim} \mathcal{D} \mathcal{P}_{p-m+l}^{m-l}(S)=\left(\begin{array}{c}
m \\
l
\end{array}\right)\left(\begin{array}{c}
p+l \\
m
\end{array}\right)=\operatorname{dim} \mathcal{X}_{p}^{l}(S) .
$$

Moreover, for $l=m$ we calculate $\operatorname{dim} \mathcal{D} \mathcal{P}_{p}^{0}(S)=\operatorname{dim} \mathcal{X}_{p}^{m}(S)+\operatorname{dim} \mathcal{W}_{0}^{m}(S)$. The agreement of dimensions has an important consequence.

Corollary 3.9. (Unisolvence of higher moments) Let $N=\operatorname{dim} \mathcal{X}_{p}^{l}(S)$ and $\left\{\beta_{1}, \ldots, \beta_{N}\right\}$ be any bases of $\mathcal{D P}_{p-m+l}^{m-l}(S)$. The functionals

$$
\eta_{i}: \mathcal{X}_{p}^{l}(S) \mapsto \mathbb{R}, \quad \omega \mapsto \int_{S} \omega \wedge \beta_{i}, \quad i=1, \ldots, N
$$


provide a dual basis of $\mathcal{X}_{p}^{l}(S)$ for $l<m$, or $\mathcal{X}_{p}^{m}(S)+\mathcal{W}_{0}^{m}(S)$ for $l=m$. Conversely, any set of degrees of freedom for $\mathcal{X}_{p}^{l}(S), l<m$, or $\mathcal{X}_{p}^{m}(S)+$ $\mathcal{W}_{0}^{m}(S)$, respectively, has the above representation.

The degrees of freedom are integrals of an $l$-form weighted with polynomials. This is why they are called moment-based.

Given degrees of freedom on $\mathcal{W}_{\underline{\mathbf{p}}}^{l}\left(\Omega_{h}\right)$ that satisfy (i) and (ii), we can define the moment-based interpolation operators $\Pi_{\underline{\mathbf{p}}}^{l}: \mathcal{F}^{l}(\Omega) \mapsto \mathcal{W}_{\underline{\mathbf{p}}}^{l}\left(\Omega_{h}\right)$ by

$$
\kappa\left(\omega-\Pi_{\underline{\mathbf{p}}}^{l} \omega\right)=0 \quad \forall \kappa \in \Theta_{\underline{\mathbf{p}}}^{l}\left(\Omega_{h}\right) .
$$

An important property of the interpolation operators $\Pi^{l}$ for Whitney forms is commuting diagram property (3.12). If we want this to carry over to the variable $p$-version of discrete differential forms, we have to impose the following minimum degree rule on the local polynomial degrees $p_{S}$ :

$$
\forall S \in \mathcal{S}_{m}\left(\Omega_{h}\right), l \leq m \leq n: p_{S} \leq \min \left\{p_{R}, R \in \mathcal{S}_{m-1}\left(\Omega_{h}\right), R \subset \partial S\right\}
$$

Theorem 3.10. (Commuting diagram property) If $\underline{\mathbf{p}}$ complies with (3.32), any moment-based local interpolation operator according to (3.31) satisfies the commuting diagram property

$$
\boldsymbol{d} \circ \Pi_{\underline{\mathbf{p}}}^{l}=\Pi_{\underline{\mathbf{p}}}^{l+1} \circ \boldsymbol{d} .
$$

Proof. For $\omega \in \mathcal{D F}^{l, 0}(\Omega)$, integration by parts yields

$$
\int_{S} \boldsymbol{d}\left(\omega-\Pi_{\underline{\mathbf{p}}}^{l} \omega\right) \wedge \mu= \pm \int_{S}\left(\operatorname{Id}-\Pi_{\underline{\mathbf{p}}}^{l}\right) \omega \wedge \boldsymbol{d} \mu+\int_{\partial S}\left(\operatorname{Id}-\Pi_{\underline{\mathbf{p}}}^{l}\right) \omega \wedge \mu .
$$

If $\mu \in \mathcal{D} \mathcal{P}_{p+1-m+l}^{m-1-l}(S)$, the integrals on the right-hand side represent valid functionals (in $\omega$ ) for the definition of $\Pi_{\mathbf{p}}^{l}$. Hence, by (3.31) the entire righthand side evaluates to zero. Observing that $\boldsymbol{d} \mathcal{W}_{\mathbf{p}}^{l}\left(\Omega_{h}\right) \in \mathcal{W}_{\mathbf{p}}^{l+1}\left(\Omega_{h}\right)$ gives the assertion.

The moment-based local interpolation operators are perfectly suitable for discrete differential forms with uniform polynomial degree. Yet the rule (3.32) is just the opposite of what is usually demanded for variable $p$-version finite element schemes, namely

$$
\forall S \in \mathcal{S}_{m}\left(\Omega_{h}\right), l \leq m \leq n: \quad p_{S} \leq \min \left\{p_{T}, T \in \mathcal{S}_{n}\left(\Omega_{h}\right), S \subset \partial T\right\}
$$

Hence, we will need another class of local projection-based interpolation operators, also denoted by $\Pi_{\mathbf{p}}^{l}$, for which the commuting diagram property remains true, even if the local polynomial degrees are chosen according to (3.33). We give their construction for the first family on simplices and assume non-uniform polynomial degrees $\mathbf{p}$. Thanks to locality, it is sufficient 
to specify $\Pi_{\mathbf{p}}^{l}$ on a single element $T$. The following tools are required for all $S \in \mathcal{S}_{m}(T), m \geq l$.

- Liftings $\mathrm{PL}_{S}^{l}:\left\{\omega \in \mathcal{X}_{p_{S}}^{l}(S), \boldsymbol{d} \omega=0\right\} \mapsto \mathcal{X}_{p_{S}}^{l-1}(S)$ such that $\boldsymbol{d} \circ \mathrm{PL}_{S}^{l}=$ Id. An example of such a lifting was explicitly constructed in the proof of Lemma 3.5. It should be remarked that the liftings $\mathrm{PL}_{S}^{l}$ have to be compatible with the choice of spaces $\mathcal{Y}_{p_{S}+1}^{l}(S)$. Conversely, their ranges can even be used to fix these spaces. This approach was pursued in Hiptmair (1999a) and (2001c).

- Extension operators $\mathrm{PE}_{S}^{l}: \mathcal{X}_{p_{S}}^{l}(S) \mapsto \mathcal{W}_{p_{T}}^{l}(T)$ with $\mathbf{t}_{S^{\prime}} \circ \mathrm{PE}_{S}^{l}=0$ for all $S^{\prime} \in \mathcal{S}_{m}(S) \backslash\{S\}$. Moreover, the choice of extension operators on different cells has to take into account the patch condition (2.20). Examples include barycentric extensions, but there are many choices.

- Projections $\mathrm{PQ}_{S}^{l}$ from $l$-forms on $S$ onto $\left\{\omega \in \mathcal{X}_{p_{S}}^{l}(S), \boldsymbol{d} \omega=0\right\}$.

Then, given $\omega \in \mathcal{D} \mathcal{F}^{l, 0}(T)$ its interpolant $\tau_{h}:=\Pi_{\mathbf{p}}^{l} \omega$ on $T$ is defined by the algorithm of Figure 3.5. Observe that, for $l=0$, the extensions are the only relevant mappings and Figure 3.5 describes the common interpolation procedure for $p$-version Lagrangian finite elements.

Lemma 3.11. The projection-based interpolation operators

$$
\Pi_{\underline{\mathbf{p}}}^{l}: \mathcal{D F}^{l, 0}(T) \mapsto \mathcal{W}_{\underline{\mathbf{p}}}^{l}(T)
$$

are linear projections and possess the commuting diagram property.

Proof. Both assertions of the theorem can be established by simple induction arguments inspired by the recursive definition of $\Pi_{\underline{p}}^{l}$ in Figure 3.5.

$$
\begin{aligned}
& \omega \leftarrow \omega-\Pi^{l} \omega ; \quad \tau_{h}:=\Pi^{l} \omega ; \\
& \text { for }(m=l ; m \leq n ; m++) \\
& \{ \\
& \text { foreach }\left(S \in \mathcal{S}_{m}(T)\right) \\
& \{ \\
& \xi_{h}:=\mathrm{PL}_{S}^{l+1}\left(\mathrm{PQ}_{S}^{l+1} \boldsymbol{d}\left(\mathbf{t}_{S} \omega\right)\right) ; \\
& \eta_{h}:=\mathrm{PL}_{S}^{l}\left(\mathrm{PQ}_{S}^{l}\left(\mathbf{t}_{S} \omega-\xi_{h}\right)\right) ; \\
& \zeta_{h}:=\mathrm{PE}_{S}^{l} \xi_{h}+\boldsymbol{d}\left(\mathrm{PE}_{S}^{l-1} \eta_{h}\right) ; \\
& \} \\
& \omega \leftarrow \omega-\zeta_{h} ; \quad \tau_{h} \leftarrow \tau_{h}+\zeta_{h} ; \\
& \}
\end{aligned}
$$

Figure 3.5. Algorithm defining the local projection-based interpolation operator $\Pi_{\underline{\mathbf{p}}}^{l}$ through the computation of $\tau_{h}:=\Pi_{\underline{\mathbf{p}}}^{l} \omega$ 
From now on we will always take for granted that the families of spaces of discrete differential forms are equipped with local interpolation operators $\Pi_{p}^{l}, \Pi_{\underline{\mathbf{p}}}^{l}$, for which the commuting diagram property holds.

\section{Bibliographical notes}

The moment-based degrees of freedom are the classical choice for spaces of discrete differential forms, when introduced as 'mixed finite elements' (Nédélec 1980, Nédélec 1986, Brezzi and Fortin 1991). The projection-based construction is due to Demkowicz et al. (Demkowicz, Monk, Schwab and Vardapetyan 1999, Monk and Demkowicz 2001, Demkowicz and Babuška 2001).

\subsection{Interpolation estimates}

Eventually discrete differential forms are meant to approximate integral forms representing physical fields. To gauge their efficacy, we study how well differential forms can be approximated by their discrete counterparts in the natural metric provided by the energy norms. Often, sufficient information can be obtained from examining the interpolation error for the local projections. By and large, this can be accomplished drawing on techniques developed for Lagrangian finite elements.

Due to their relevance for electromagnetism, we will largely restrict our attention to 1-forms in three dimensions. In addition, we will chiefly consider discrete forms on tetrahedral triangulations. Approximation by discrete differential forms can be enhanced in two ways:

(1) by using finer meshes (h-version of discrete differential forms), and

(2) by raising the polynomial degree $p$ on a fixed mesh ( $p$-version of discrete differential forms).

The $h$-version of discrete differential forms, that is, the case of fixed uniform polynomial degree $p \in \mathbb{N}_{0}$ will be investigated first. In this case momentbased local interpolation operators are natural. Following the classical approach (Ciarlet 1978, Section 3.1), for a mesh $\Omega_{h}$ we introduce its shape regularity measure by

$$
\rho\left(\Omega_{h}\right):=\max _{T \in \mathcal{S}_{3}\left(\Omega_{h}\right)} \rho_{T}, \quad \rho_{T}:=h_{T} / r_{T},
$$

where $h_{T}:=\max \{|\mathbf{x}-\mathbf{y}|, \mathbf{x}, \mathbf{y} \in T\}$ is the diameter of a cell $T$, and $r_{T}:=$ $\max \{\exists \mathbf{x} \in T:|\mathbf{x}-\mathbf{y}| \leq \rho \Rightarrow \mathbf{y} \in T\}$ is the radius of the largest inscribed ball. We remark that $\rho\left(\Omega_{h}\right)$ can be computed from bounds for the largest and smallest angles enclosed by faces of the tetrahedra. From now on the index $h$ of $\Omega_{h}$ should be read as meshwidth

$$
h:=\max \left\{h_{T}, T \in \mathcal{S}_{3}\left(\Omega_{h}\right)\right\} .
$$


Techniques based on the pullback of differential forms are instrumental in getting interpolation estimates. They are available if the spaces of discrete differential forms in $\Omega_{h}$ are affine-equivalent in the following sense. There is a reference simplex $\widehat{T}$ such that the local space on any $T \in \mathcal{S}_{3}\left(\Omega_{h}\right)$ can be obtained from that on $\widehat{T}$ via affine pullback ( $c f .(2.16)-(2.19))$

$$
\mathcal{W}_{p}^{l}(T)=\left(\mathbf{\Phi}_{T}^{-1}\right)^{*} \mathcal{W}_{p}^{l}(\widehat{T}),
$$

where $\boldsymbol{\Phi}_{T}: \widehat{T} \mapsto T, \boldsymbol{\Phi}(\widehat{\mathbf{x}}):=\mathrm{B}_{T} \widehat{\mathbf{x}}+\mathbf{t}_{T}, \mathrm{~B}_{T} \in \mathbb{R}^{3,3}$ regular, $\mathbf{t}_{T} \in \mathbb{R}^{3}$, is the unique affine mapping taking $\widehat{T}$ to $T$. Moreover, the local projections on $\widehat{T}$ and affine pullbacks must commute:

$$
\Pi_{p \mid T}^{l} \circ\left(\mathbf{\Phi}_{T}^{-1}\right)^{*}=\left(\boldsymbol{\Phi}_{T}^{-1}\right)^{*} \circ \Pi_{p \mid \widehat{T}^{*}}^{l}
$$

We saw in Section 3.2 that Whitney forms are affine-equivalent. For higherorder discrete differential forms this can be ensured by first constructing the local spaces on a reference element. Afterwards (3.35) is used to get all local spaces. The relationship (3.36) is enforced by using affine pullbacks, too, in order to assemble the local interpolation operators from those on $\widehat{T}$ ( $c f$. the discussion in Section 4 of Hiptmair (2001c)).

For the rest of the article we fix $\widehat{T}$ to be the customary reference tetrahedron spanned by the canonical basis vectors in Euclidean space $\mathbb{R}^{3}$. Using this, we find, for every $T \in \mathcal{S}_{3}\left(\Omega_{h}\right)$ (Ciarlet 1978, Theorem 3.1.3),

$$
\begin{gathered}
\left\|\mathrm{B}_{T}\right\| \leq 4 \rho_{T} h_{T}, \quad\left\|\mathrm{~B}_{T}^{-1}\right\| \leq \rho_{T} h_{T}^{-1}, \\
\left|\operatorname{det} \mathrm{B}_{T}\right| \leq 6 h_{T}^{3}, \quad\left|\operatorname{det} \mathrm{B}_{T}\right|^{-1} \leq \frac{1}{8 \pi} \rho_{T} h_{T}^{-3},
\end{gathered}
$$

with $\left\|\mathrm{B}_{T}\right\|$ standing for the Euclidean matrix norm. The gist of affine equivalence techniques is to use these estimates in combination with the pullback formulas (2.16)-(2.19) for vector proxies. Thanks to (3.36) any local interpolation error can then be bounded in terms of interpolation errors on $\widehat{T}$.

The estimates of interpolation errors on $\widehat{T}$ rely on extra smoothness of the fields, which is measured by certain Sobolev (semi-)norms of the (components of the) vector proxies. For a vector proxy $\mathbf{u}=\left(u_{1}, \ldots, u_{K}\right), K \in \mathbb{N}$, they read

$$
\begin{aligned}
|\mathbf{u}|_{H^{m}(\Omega)}^{2} & :=\sum_{k=1}^{K} \int_{\Omega}\left|D^{m} u_{k}(\mathbf{x})\right|^{2} \mathrm{~d} \mathbf{x}, \quad m \in \mathbb{N}_{0}, \\
|\mathbf{u}|_{H^{s}(\Omega)}^{2} & :=\sum_{k=1}^{K} \int_{\Omega} \int_{\Omega} \frac{\left|u_{k}(\mathbf{x})-u_{k}(\mathbf{y})\right|^{2}}{|\mathbf{x}-\mathbf{y}|^{3+2 s}} \mathrm{~d} \mathbf{x} \mathrm{d} \mathbf{y}, \quad 0 \leq s<1 .
\end{aligned}
$$

The associated Sobolev spaces of vector proxies are $\boldsymbol{H}^{m}(\Omega)$ and $\boldsymbol{H}^{s}(\Omega)$. The behaviour of these norms under the pullbacks given by $(2.16)-(2.18)$ 
has to be examined. We include the well-known estimates for 0-forms in order to highlight the pattern.

Lemma 3.12. (Transformation of norms under pullbacks) Let $T=$ $\boldsymbol{\Phi}_{T}(\widehat{T})$ be the image of the reference tetrahedron under the bijective affine mapping $\boldsymbol{\Phi}_{T}, \boldsymbol{\Phi}_{T}(\widehat{\mathbf{x}}):=\mathrm{B}_{T} \widehat{\mathbf{x}}+\mathbf{t}_{T}$ as above. Then, for all $m \in \mathbb{N}_{0}$ and all vector-fields/functions in $\boldsymbol{H}^{m}(T) / H^{m}(T)$,

$$
\begin{aligned}
&\left|\mathfrak{F}_{\boldsymbol{\Phi}_{T}}^{0} v\right|_{H^{m}(\widehat{T})}^{2} \leq\left\|\mathrm{B}_{T}\right\|^{2 m}\left|\operatorname{det} \mathrm{B}_{T}\right|^{-1}|v|_{H^{m}(T)}^{2} \leq C \rho_{T} h_{T}^{2 m-3}|v|_{H^{m}(T)}^{2}, \\
&\left|\mathfrak{F}_{\boldsymbol{\Phi}_{T}}^{1} \mathbf{u}\right|_{\boldsymbol{H}^{m}(\widehat{T})}^{2} \leq\left\|\mathrm{B}_{T}\right\|^{2+2 m}\left|\operatorname{det} \mathrm{B}_{T}\right|^{-1}|\mathbf{u}|_{\boldsymbol{H}^{m}(T)}^{2} \leq C \rho_{T} h_{T}^{2 m-1}|\mathbf{u}|_{\boldsymbol{H}^{m}(T)}^{2} \\
&\left|\mathfrak{F}_{\boldsymbol{\Phi}_{T}}^{2} \mathbf{u}\right|_{\boldsymbol{H}^{m}(\widehat{T})}^{2} \leq\left\|\mathrm{B}_{T}\right\|^{2 m}\left\|\mathrm{~B}_{T}^{-1}\right\|^{2}\left|\operatorname{det} \mathrm{B}_{T}\right||\mathbf{u}|_{\boldsymbol{H}^{m}(T)}^{2} \leq C \rho_{T} h_{T}^{2 m+1}|\mathbf{u}|_{\boldsymbol{H}^{m}(T)}^{2}
\end{aligned}
$$

with universal constants $C>0$. For any $0 \leq s<1$, we have

$$
\begin{aligned}
\left|\mathfrak{F}_{\boldsymbol{\Phi}_{T}}^{0} v\right|_{H^{s}(\widehat{T})}^{2} & \leq\left\|\mathrm{B}_{T}\right\|^{2 s+3}\left|\operatorname{det} \mathrm{B}_{T}\right|^{-2}|v|_{H^{s}(T)}^{2} \leq C \rho_{T} h_{T}^{2 s-3}|v|_{H^{s}(T)}^{2}, \\
\left|\mathfrak{F}_{\boldsymbol{\Phi}_{T}}^{1} \mathbf{u}\right|_{\boldsymbol{H}^{s}(\widehat{T})}^{2} & \leq\left\|\mathrm{B}_{T}\right\|^{2 s+5}\left|\operatorname{det} \mathrm{B}_{T}\right|^{-2}|\mathbf{u}|_{\boldsymbol{H}^{s}(T)}^{2} \leq C \rho_{T} h_{T}^{2 s-1}|\mathbf{u}|_{\boldsymbol{H}^{s}(T)}^{2}, \\
\left|\mathfrak{F}_{\boldsymbol{\Phi}_{T}}^{2} \mathbf{u}\right|_{\boldsymbol{H}^{s}(\widehat{T})}^{2} & \leq\left\|\mathrm{B}_{T}\right\|^{2 s+3}\left\|\mathrm{~B}_{T}^{-1}\right\|^{2}|\mathbf{u}|_{\boldsymbol{H}^{s}(T)}^{2} \leq C \rho_{T} h_{T}^{2 s+1}|\mathbf{u}|_{\boldsymbol{H}^{s}(T)}^{2},
\end{aligned}
$$

for all functions/vector-fields in $\boldsymbol{H}^{s}(T)$, where $C>0$ are universal constants. $^{7}$

Proof. Using the pullback formulas (2.16)-(2.18), the definitions of the norms, and the transformation formulas for integrals, everything reduces to elementary calculations. For the fractional Sobolev norms the trick is to use

$$
|\mathbf{x}-\mathbf{y}|=\left|\mathrm{B}_{T} \mathrm{~B}_{T}^{-1}(\mathbf{x}-\mathbf{y})\right| \leq\left\|\mathrm{B}_{T}\right\|\left|\mathrm{B}_{T}^{-1}(\mathbf{x}-\mathbf{y})\right| .
$$

The details can be looked up in Ciarlet (1978, Theorem 3.1.2), Alonso and Valli (1999, Section 5), and Ciarlet, Jr, and Zou (1999, Section 3).

A first application of Lemma 3.12 confirms the $h$-uniform $L^{2}$-stability of local bases of $\mathcal{W}_{p}^{l}\left(\Omega_{h}\right)$. If the local shape functions on any $T \in \mathcal{S}_{3}\left(\Omega_{h}\right)$ are obtained by means of affine pullback from those on $\widehat{T}$, we find, for all $\alpha_{\mathbf{b}} \in \mathbb{C}$

$$
C \sum_{\mathbf{b}} \alpha_{\mathbf{b}}^{2}\|\mathbf{b}\|_{\mathbf{L}^{2}(\Omega)}^{2} \leq\left\|\sum_{\mathbf{b}} \alpha_{\mathbf{b}} \mathbf{b}\right\|_{\boldsymbol{L}^{2}(\Omega)}^{2} \leq C \sum_{\mathbf{b}} \alpha_{\mathbf{b}}^{2}\|\mathbf{b}\|_{\boldsymbol{L}^{2}(\Omega)}^{2},
$$

7 Most of the estimates will be asymptotic in nature, featuring 'generic constants', for which the symbol $C$, sometimes tagged with a subscript, is used throughout. The value of these generic constants may vary between different occurrences, but it will always be made clear what they depend upon. When the $h$-version of discrete differential forms is considered, the constants may not depend on meshwidth. 
where $\mathbf{b}$ runs through the set of basis functions of $\mathcal{W}_{p}^{l}\left(\Omega_{h}\right)$ and the constants depend on $p$ and $\rho\left(\Omega_{h}\right)$ only. For 0 -form the constants do not even depend on $\rho\left(\Omega_{h}\right)$, but this can not be expected for $l>0$. The transformation rules of Lemma 3.12 also establish a Bernstein-type inverse estimate for discrete 1-forms: for all $\mathbf{u}_{h} \in \mathcal{W}_{p}^{1}\left(\Omega_{h}\right)$ we find

$$
\left\|\operatorname{curl} \mathbf{u}_{h}\right\|_{\boldsymbol{L}^{2}(\Omega)} \leq C h^{-1}\left\|\mathbf{u}_{h}\right\|_{\boldsymbol{L}^{2}(\Omega)}
$$

with $C=C\left(\rho\left(\Omega_{h}\right)\right)>0$.

Affine equivalence techniques owe their power to Bramble-Hilbert arguments on the reference element (Ciarlet 1978, Section 3.1). These are available if, firstly, the interpolation operators $\widehat{\Pi}_{p}^{l}$ on $\widehat{T}$ preserve spaces of polynomials. Secondly, they have to be continuous on spaces of sufficiently smooth functions. The latter requirement is addressed by the following lemma ( cf. Lemma 4.7 in Amrouche et al. (1998)).

Lemma 3.13. (Continuity of edge moments) Let $\hat{e}$ be an edge of the reference element $\widehat{T}$ and $\hat{F}$ be a face adjacent to $\hat{e}$. If $\varphi \in W_{q}^{1-1 / q}(\hat{e})$ with $1<q<2$, then, for $p^{-1}+q^{-1}=1$, any smooth vector-field $\mathbf{u}$ on $\widehat{T}$, and $C=C(p)>0$,

$$
\left|\int_{\hat{e}} \varphi \mathbf{u} \cdot \mathrm{d} \mathbf{s}\right| \leq C\left(\|\mathbf{c u r l} \mathbf{u}\|_{L^{p}(\widehat{T})}+\left\|\gamma_{\mathbf{t}} \mathbf{u}\right\|_{L^{p}(\hat{F})}\right)\|\varphi\|_{W_{q}^{1-1 / q}(\hat{e})} .
$$

Proof. Denote by $\bar{\varphi}$ the extension by zero of $\varphi$ to $\partial \hat{F}$. As smooth functions with compact support are dense in $W_{q}^{1-1 / q}(\hat{e})$, we also have $\bar{\varphi} \in$ $W_{q}^{1-1 / q}(\partial \hat{F})$. By Theorem 1.5.1.3 in Grisvard (1985) we can extend $\bar{\varphi}$ to a function $\hat{\varphi} \in W_{q}^{1}(\hat{F})$ in a stable fashion. Again, $\hat{\varphi}$ can be extended by zero onto $\partial \widehat{T}$, which yields a function $\tilde{\varphi} \in W_{q}^{1-1 / q}(\partial \widehat{T})$. Once more appealing to Theorem 1.5.1.3 in Grisvard (1985), we extend $\tilde{\varphi}$ to $\check{\varphi} \in W_{q}^{1}(\widehat{T})$. The continuity of these extensions is reflected by the norm estimates

$$
\begin{aligned}
\|\check{\varphi}\|_{W_{q}^{1}(\widehat{T})} & \leq C\|\tilde{\varphi}\|_{W_{q}^{1-1 / q}(\partial \widehat{T})} \leq C\|\hat{\varphi}\|_{W_{q}^{1}(\hat{F})} \\
& \leq C\|\bar{\varphi}\|_{W_{q}^{1-1 / q}(\partial \hat{F})} \leq C\|\varphi\|_{W_{q}^{1-1 / q}(\hat{e})},
\end{aligned}
$$

where $C$ stands for generic positive constants depending only on $p$. Next, we use integration by parts, more precisely Stokes' theorem on $\hat{F}$ combined with Green's formula in $\widehat{T}$ :

$$
\begin{aligned}
\int_{\hat{e}} \varphi \mathbf{u} \cdot \mathrm{d} \mathbf{s} & =\int_{\partial \hat{F}} \bar{\varphi} \mathbf{u} \cdot \mathrm{d} \mathbf{s}=\int_{\hat{F}} \operatorname{curl}(\mathbf{u} \bar{\varphi}) \cdot \mathbf{n} \mathrm{d} S \\
& =\int_{\hat{F}} \hat{\varphi} \operatorname{curl} \mathbf{u} \cdot \mathbf{n} \mathrm{d} S-\int_{\hat{F}}(\mathbf{u} \times \operatorname{grad} \hat{\varphi}) \cdot \mathbf{n} \mathrm{d} S
\end{aligned}
$$




$$
\begin{aligned}
& =\int_{\partial \widehat{T}} \tilde{\varphi} \operatorname{curl} \mathbf{u} \cdot \mathbf{n} \mathrm{d} S+\int_{\hat{F}} \operatorname{grad} \hat{\varphi} \cdot(\mathbf{u} \times \mathbf{n}) \mathrm{d} S \\
& =\int_{\widehat{T}} \operatorname{grad} \check{\varphi} \cdot \operatorname{curl} \mathbf{u} \mathrm{d} \mathbf{x}+\int_{\hat{F}} \operatorname{grad} \hat{\varphi} \cdot(\mathbf{u} \times \mathbf{n}) \mathrm{d} S .
\end{aligned}
$$

Thus, by Hölder's inequality,

$$
\left|\int_{\hat{e}} \varphi \mathbf{u} \cdot \mathrm{d} \mathbf{s}\right| \leq\|\check{\varphi}\|_{W_{q}^{1}(\widehat{T})}\|\operatorname{curl} \mathbf{u}\|_{L^{p}(\widehat{T})}+\|\hat{\varphi}\|_{W_{q}^{1}(\hat{F})}\left\|\gamma_{\mathbf{t}} \mathbf{u}\right\|_{L^{p}(\hat{F})} .
$$

Along with the stability of the extensions this gives the result.

Transformations of $L^{p}$-norms are awkward to handle. Thus it is desirable to switch back to Sobolev spaces $\boldsymbol{H}^{s}(\widehat{T})$, for which Lemma 3.12 provides transformation rules. This can be achieved by means of the continuous embedding (Adams 1975, Section 5.4)

$$
H^{s}(\widehat{T}) \hookrightarrow L^{\frac{6}{3-2 s}}(\widehat{T}), \quad 0 \leq s<\frac{3}{2},
$$

and the continuity of the tangential trace mappings

$$
\left(\gamma_{\mathbf{t}}\right)_{\mid \hat{F}}: \boldsymbol{H}^{\frac{1}{2}+s}(\widehat{T}) \mapsto \boldsymbol{H}^{s}(\hat{F}), \quad s>0,
$$

for each face $\hat{F}$ of $\widehat{T}$. Recall that the moment-based interpolation operators for discrete 1-forms rely on weighted path integrals along edges, moments of tangential traces on faces and weighted integrals over cells. The weights are fixed polynomials. For $s>\frac{1}{2}$ the continuity on $\boldsymbol{H}^{s}(\widehat{T})$ of d.o.f. associated with faces and cells is straightforward. This gives continuity

$$
\left\|\widehat{\Pi}_{p}^{1} \mathbf{u}\right\|_{\boldsymbol{L}^{2}(\widehat{T})} \leq C\left(\|\mathbf{u}\|_{\boldsymbol{H}^{s}(\widehat{T})}+\|\operatorname{curl} \mathbf{u}\|_{\boldsymbol{H}^{s-\frac{1}{2}(\widehat{T})}}\right)
$$

for all sufficiently smooth $\mathbf{u}, s>\frac{1}{2}$, with $C>0$ depending only on $s$ and $p$.

Theorem 3.14. (Moment-based interpolation estimates for discrete 1-forms) For any tetrahedral mesh $\Omega_{h}$ with meshwidth $\left.h>0, s \in\right] \frac{1}{2} ; 1[\cup \mathbb{N}$, and affine equivalent discrete 1 -forms $\mathcal{W}_{p}^{1}\left(\Omega_{h}\right)$ of uniform polynomial degree $p \in \mathbb{N}_{0}$, we have the interpolation error estimate

$$
\left\|\mathbf{u}-\Pi_{p}^{1} \mathbf{u}\right\|_{\boldsymbol{L}^{2}(\Omega)} \leq C h^{\min \{s, p+1\}}\left(\|\mathbf{u}\|_{\boldsymbol{H}^{s}(\Omega)}+\|\operatorname{curl} \mathbf{u}\|_{\boldsymbol{H}^{s}(\Omega)}\right)
$$

for all sufficiently smooth vector-fields $\mathbf{u}$, with $C>0$ depending only on the shape regularity measure of the mesh $\Omega_{h}, s$, and the specification of $\widehat{\Pi}_{p}^{1}$.

Proof. First, we treat the case $s=m \in \mathbb{N}$. Pick any $T \in \mathcal{S}_{3}\left(\Omega_{h}\right)$. Then use (3.36) and the transformation rule for the $\boldsymbol{L}^{2}(T)$-norm of 1 -forms from 
Lemma 3.12 and note that $\mathcal{W}_{p}^{1}(\widehat{T})$ contains all polynomials of degree $\leq p$. This gives

$$
\begin{aligned}
\| \mathbf{u} & -\Pi_{p \mid T}^{1} \mathbf{u} \|_{\boldsymbol{L}^{2}(T)}^{2} \\
& \leq C \rho\left(\Omega_{h}\right) h_{T}\left\|\mathfrak{F}_{\boldsymbol{\Phi}_{T}}^{1}\left(\mathbf{u}-\widehat{\Pi}_{p}^{1} \mathbf{u}\right)\right\|_{\boldsymbol{L}^{2}(\widehat{T})}^{2} \\
& \leq C \rho\left(\Omega_{h}\right) h_{T}\left\|\left(\operatorname{Id}-\widehat{\Pi}_{p}^{1}\right) \widehat{\mathbf{u}}\right\|_{\boldsymbol{L}^{2}(\widehat{T})}^{2} \\
& \leq C \rho\left(\Omega_{h}\right) h_{T} \inf _{\mathbf{p} \in\left(\mathcal{P}_{p}(\widehat{T})\right)^{3}}\left(\|\widehat{\mathbf{u}}-\mathbf{p}\|_{\boldsymbol{H}^{m^{\prime}}(\widehat{T})}^{2}+\|\operatorname{curl}(\widehat{\mathbf{u}}-\mathbf{p})\|_{\boldsymbol{H}^{r-\frac{1}{2}}(\widehat{T})}^{2}\right) \\
& \leq C \rho\left(\Omega_{h}\right) h_{T}\left(|\widehat{\mathbf{u}}|_{\boldsymbol{H}^{m^{\prime}}(\widehat{T})}^{2}+|\mathbf{c u r l} \widehat{\mathbf{u}}|_{\boldsymbol{H}^{m^{\prime}}(\widehat{T})}^{2}\right)
\end{aligned}
$$

for $\widehat{\mathbf{u}}:=\mathfrak{F}_{\boldsymbol{\Phi}_{T}}^{1} \mathbf{u}, m^{\prime}=\min \{m, p+1\}$, and constants depending only on $p$ and $m$. The final step rests on the Peetre-Tartar lemma (Girault and Raviart 1986, Theorem 2.1), which shows the existence of $C=C\left(m^{\prime}\right)>0$, such that, for all smooth $\widehat{\mathbf{u}}$,

$$
\begin{aligned}
& \inf _{\mathbf{p} \in(\widehat{\mathcal{P}}(\widehat{T}))^{3}}\left(\|\widehat{\mathbf{u}}-\mathbf{p}\|_{\boldsymbol{H}^{m^{\prime}(\widehat{T})}}+\|\mathbf{c u r l}(\widehat{\mathbf{u}}-\mathbf{p})\|_{\boldsymbol{H}^{m^{\prime}(\widehat{T})}}\right) \\
& \leq C\left(|\widehat{\mathbf{u}}|_{\boldsymbol{H}^{m^{\prime}(\widehat{T})}}+|\operatorname{curl} \widehat{\mathbf{u}}|_{\boldsymbol{H}^{m^{\prime}(\widehat{T})}}\right) .
\end{aligned}
$$

The remaining semi-norms have to be taken back to $T$ using the transformation rules of 1 -forms for $\mathbf{u}$ and those for 2 -forms for $\mathbf{c u r l} \mathbf{u}$. This gives

$$
\left\|\mathbf{u}-\Pi_{p \mid T}^{1} \mathbf{u}\right\|_{\boldsymbol{L}^{2}(T)}^{2} \leq C \rho\left(\Omega_{h}\right) h_{T}^{2 m^{\prime}}\left(|\mathbf{u}|_{\boldsymbol{H}^{m^{\prime}(T)}}^{2}+|\mathbf{c u r l} \mathbf{u}|_{\boldsymbol{H}^{m^{\prime}(T)}}^{2}\right),
$$

and the assertion for integer $s$ follows by adding these estimates for all cells.

If $\frac{1}{2}<s<1$, we adapt the above arguments and find

$$
\begin{aligned}
& \left\|\mathbf{u}-\Pi_{p_{\mid T}}^{1} \mathbf{u}\right\|_{\boldsymbol{L}^{2}(T)}^{2} \\
& \quad \leq C \inf _{\mathbf{p} \in\left(\mathcal{P}_{0}(\widehat{T})\right)^{3}}\left(\|\widehat{\mathbf{u}}-\mathbf{p}\|_{\boldsymbol{H}^{s}(\widehat{T})}^{2}+\|\mathbf{c u r l} \widehat{\mathbf{u}}\|_{\boldsymbol{H}^{s-\frac{1}{2}(\widehat{T})}}^{2}\right) \\
& \quad \leq C \rho\left(\Omega_{h}\right) h_{T}\left(|\widehat{\mathbf{u}}|_{\boldsymbol{H}^{s}(\widehat{T})}^{2}+\|\mathbf{c u r l} \widehat{\mathbf{u}}\|_{\boldsymbol{L}^{2}(\widehat{T})}^{2}+|\operatorname{curl} \widehat{\mathbf{u}}|_{\boldsymbol{H}^{s-\frac{1}{2}(\widehat{T})}}^{2}\right),
\end{aligned}
$$

where a Bramble-Hilbert argument in fractional Sobolev spaces was invoked (Dupont and Scott 1980, Proposition 6.1). Then an application of Lemma 3.12 finishes the proof.

In a similar fashion to Lemma 3.13, the following result can be established.

Lemma 3.15. (Continuity of face moments) For a face $\hat{F}$ of the reference element $\widehat{T}$ and $\varphi \in W_{q}^{1-1 / q}(\hat{F})$ with $1<q<2$, we have

$$
\left|\int_{\hat{F}} \varphi \mathbf{u} \cdot \mathbf{n} \mathrm{d} S\right| \leq C\left(\|\mathbf{u}\|_{\boldsymbol{L}^{p}(\widehat{T})}+\|\operatorname{div} \mathbf{u}\|_{L^{2}(\widehat{T})}\right)\|\varphi\|_{W_{q}^{1-1 / q}(\hat{F})},
$$


for any smooth vector-field $\mathbf{u}$ on $\widehat{T}$, and $C=C(p)>0$.

From this lemma we conclude the continuity

$$
\left\|\widehat{\Pi}_{p}^{2} \mathbf{u}\right\|_{\boldsymbol{L}^{2}(\widehat{T})} \leq C\left(\|\mathbf{u}\|_{\boldsymbol{H}^{s}(\widehat{T})}+\|\operatorname{div} \mathbf{u}\|_{L^{2}(\widehat{T})}\right)
$$

for all $s>0$ and $C=C(s, p)>0$. In the same fashion as Theorem 3.14, we can establish the following interpolation error estimate.

Theorem 3.16. (Moment-based interpolation estimate for discrete 2-forms) Let $\Omega_{h}$ be a tetrahedral mesh with meshwidth $h$, and $\Pi_{p}^{2}$ the local projection onto a space $\mathcal{W}_{p}^{2}\left(\Omega_{h}\right)$ belonging to the first family of discrete 2 forms on $\Omega_{h}$. Then

$$
\left\|\mathbf{u}-\Pi_{p}^{2} \mathbf{u}\right\|_{\boldsymbol{L}^{2}(\Omega)} \leq C h^{\min \{s, p+1\}}\left(\|\mathbf{u}\|_{\boldsymbol{H}^{s}(\Omega)}+\|\operatorname{div} \mathbf{u}\|_{H^{\max \{0, s\}}(\Omega)}\right)
$$

for all sufficiently smooth vector-fields $\mathbf{u}, s \in] 0,1[\cup \mathbb{N}$, and with a constant $C=C\left(s, p, \rho\left(\Omega_{h}\right)\right)>0$.

This result has been included because, thanks to the commuting diagram property $\Pi_{p}^{2} \circ \mathbf{c u r l}=\mathbf{c u r l} \circ \Pi_{p}^{1}$, we can instantly infer interpolation estimates for 1-forms in the $\|\mathbf{c u r l} \cdot\|_{\boldsymbol{L}^{2}(\Omega)}$-seminorm.

Corollary 3.17. If $\mathbf{u}$ belongs to the domain of $\Pi_{p}^{1}$ and curl $\mathbf{u}$ is sufficiently smooth, we have, for all $s>0$,

$$
\left\|\operatorname{curl}\left(\mathbf{u}-\Pi_{p}^{1} \mathbf{u}\right)\right\|_{\boldsymbol{L}^{2}(\Omega)} \leq C h^{\min \{s, p+1\}}\|\operatorname{curl} \mathbf{u}\|_{\boldsymbol{H}^{s}(\Omega)}
$$

with a constant $C=C(s, p)>0$ independent of $\mathbf{u}$.

\section{Bibliographical notes}

Some more technical details on moment-based interpolation estimates can be found in Girault and Raviart (1986, Chapter 3, Section 5), Alonso and Valli (1999, Section 5), and Ciarlet, Jr, and Zou (1999, Section 3).

Remark 9. All the above estimates hinge on the shape regularity measure, which deteriorates even for tetrahedra like

$$
T=\left[(0,0,0)^{T},\left(h_{1}, 0,0\right)^{T},\left(0, h_{2}, 0\right)^{T},\left(0,0, h_{3}\right)^{T}\right]
$$

when $h_{1}, h_{2}, h_{3}$ differ strongly. We may recall the advice from Section 3.2: it is known that the usual interpolation estimates for the standard local interpolation operator for Whitney 0-forms still hold on such elements with the local interpolation error depending on $\max \left\{h_{1}, h_{2}, h_{3}\right\}$ only (Apel and Dobrowolski 1992, Apel 1999). A corresponding result was shown for Whitney 2-forms in Nicaise (2001) and Apel, Nicaise and Schöberl (2001). We may conjecture that Whitney 1-forms behave identically, but proof is at present elusive (Some preliminary investigations for edge elements have been conducted by Nicaise (2001)). 
Remark 10. For the first family of discrete differential forms we get the same order of the interpolation error in the $\boldsymbol{L}^{2}(\Omega)$-norm and the $\|\operatorname{curl} \cdot\|_{\boldsymbol{L}^{2}(\Omega)^{-}}$ seminorm. This is surprising because one would expect the application of curl to reduce one power of $h$ in the error estimate.

Remark 11. The spaces $\check{\mathcal{W}}_{p}^{l}\left(\Omega_{h}\right)$ of the second family of discrete differential forms locally contain all polynomials up to a total degree of $p+1$. Therefore, in Theorem 3.14 the order of the interpolation error can be raised to $p+2$, smoothness of $\mathbf{u}$ permitting. No improvement in the estimate of Theorem 3.16 can be achieved. Thus, the second family really displays the typical poorer decrease of the interpolation error in norms involving a differential operator. From the perspective of theoretical results it is hard to assess the gain from using the second family in Galerkin schemes: the simultaneous approximation of the vector-field and its curl will always be needed. Therefore the curl of the interpolation error $\mathbf{u}-\Pi_{p}^{1} \mathbf{u}$ will dominate in a priori error estimates ( $c f$. Sections 4 and 5).

Remark 12. For parametric elements, that is, the case of a general diffeomorphism $\boldsymbol{\Phi}_{T}: \widehat{T} \mapsto T$, apart from the infimum/supremum on $\widehat{T}$ of the expressions $\left\|D \boldsymbol{\Phi}_{T}(\widehat{\mathbf{x}})\right\|,\left|\operatorname{det} D \boldsymbol{\Phi}_{T}(\widehat{\mathbf{x}})\right|$, norms of higher derivatives of $\boldsymbol{\Phi}_{T}$ enter the bounds of Lemma 3.12. This renders the shape regularity measure an inadequate tool for the analysis of interpolation errors (Girault and Raviart (1986, Chapter I, A.2) and Ciarlet (1978, Chapter 4, Section 4.3)). We also have to impose bounds for the deviation of $\boldsymbol{\Phi}_{T}$ from an affine mapping. No investigations have yet been carried out for discrete 1-forms, but the results for Lagrangian finite elements should carry over.

For the $p$-version of discrete differential forms, the projection-based interpolation operators have to be considered. Their continuity properties are crucial. These hinge on the continuity of the mappings $\mathrm{PL}_{S}^{l}, \mathrm{PE}_{S}^{l}$, and $\mathrm{PQ}_{S}^{l}$ used for the definition of the interpolation operators in Section 3.5. We examine these separately for tetrahedral discrete 1-forms, starting with lifting mappings. As $\Omega_{h}$ is fixed and the interpolation is local, we need only scrutinize a single element $T$. All argument functions are supposed to be sufficiently smooth and the rule (3.33) is to be in effect.

- For any edge $e$ the lifting $\mathrm{PL}_{e}^{1}$ amounts to integration along $e$. We conclude that, uniformly in the polynomial degree $p$,

$$
\left\|\mathrm{PL}_{e}^{1}(u)\right\|_{L^{2}(e)} \leq C\left(\|u\|_{H^{-1}(e)}+\left|\int_{e} l(s) u(s) \mathrm{d} s\right|\right),
$$

where $l(s)$ is a linear function on $e$ vanishing at one endpoint and assuming the value 1 at the other. 
- For a triangular face $F$ and a tetrahedral cell $T$, the liftings $\mathrm{PL}_{F}^{1}$, $\mathrm{PL}_{T}^{1}$ boil down to finding scalar potentials, that is, $\operatorname{grad} \mathrm{PL}_{S}^{1} \mathbf{u}=\mathbf{u}$, $S \in\{F, T\}$, in terms of vector proxies. Imposing vanishing averages on the scalar potentials, it is elementary that, uniformly in $p$ and $\mathbf{u}$,

$$
\left\|\mathrm{PL}_{S}^{1}(\mathbf{u})\right\|_{H^{1}(S)} \leq C\|\mathbf{u}\|_{\boldsymbol{L}^{2}(S)}, \quad S \in\{F, T\} .
$$

- The lifting mappings $\mathrm{PL}_{F}^{2}, F$ face, and $\mathrm{PL}_{T}^{2}, T$ cell, for 2-forms are to yield vector potentials with zero boundary conditions. Suitable mappings can be constructed as in the proof of Lemma 3.5 based on the Poincaré mapping. The $L^{2}$-continuity of the Poincaré mapping (confirmed by applying the Cauchy-Schwarz inequality to (3.21)) combined with extension theorems for polynomials, known in the theory of $p$-version Lagrangian finite elements (Babuška, Craig, Mandel and Pitkäranta 1991, Section 7), gives

$$
\left\|\mathrm{PL}_{S}^{2}(\mathbf{u})\right\|_{\boldsymbol{L}^{2}(S)} \leq C\|\mathbf{u}\|_{\boldsymbol{L}^{2}(S)}, \quad S \in\{F, T\}
$$

with $C>0$ independent of $\mathbf{u}$ and $p$. Details in the case of faces can be found in Demkowicz and Babuška (2001, Section 3).

As mappings $\mathrm{PQ}_{e}^{1}$ we choose $H^{-1}(e)$-orthogonal projections. All the other required projections $\mathrm{PQ}_{F}^{1}, \mathrm{PQ}_{F}^{2}, F$ face, and $\mathrm{PQ}_{T}^{1}, \mathrm{PQ}_{T}^{2}, T$ cell, may be $\boldsymbol{L}^{2}(S)$-orthogonal, $S \in\{F, T\}$.

Finally, the extensions $\mathrm{PE}_{e}^{0}$, e edge, and $\mathrm{PE}_{F}^{0}, F$ face, are extensions of polynomials with zero traces on $\partial e$ and $\partial F$, respectively. We demand that they satisfy

$$
\begin{gathered}
\left\|\mathrm{PE}_{e}^{0}(q)\right\|_{H^{1}(T)} \leq C\|q\|_{L^{2}(e)} \\
\left\|\mathrm{PE}_{F}^{0}(q)\right\|_{H^{1}(T)} \leq C\|q\|_{H^{1}(F)}
\end{gathered}
$$

for all admissible polynomials $q$ and uniformly in $p$. For the extension $\mathrm{PE}_{e}^{0}$ a related result can be found in Pavarino and Widlund (1996, Section 4.4). The existence of a stable extension from the faces can be concluded from Munoz-Solar (1997, Theorem 1). One more extension $\mathrm{PE}_{F}^{1}$ of polynomial vector-fields on faces is needed. Unfortunately, no results about possible $p$-uniformly continuous constructions are available. Therefore we make the assumption that, for all $p$ and $\mathbf{u} \in \mathcal{X}_{p}^{1}(F)$,

$$
\left\|\operatorname{PE}_{F}^{1}(\mathbf{u})\right\|_{\boldsymbol{L}^{2}(T)} \leq C\|\mathbf{u}\|_{\boldsymbol{L}^{2}(F)} .
$$

From Adams (1975, Theorem 7.58) we learn the continuous embedding of $H^{1}(I)$ into $W_{q}^{1-1 / q}(I), 1 \leq q \leq 2$, for a bounded interval $I \subset \mathbb{R}$. Recalling Lemma 3.13, we conclude, for any edge $e$ of $T$, sufficiently smooth 
vector-fields $\mathbf{u}$, and $q>2$, that

$$
\left\|\mathbf{u} \cdot \mathbf{t}_{e}\right\|_{H^{-1}(e)}+\left|\int_{e} l(s) u(s) \mathrm{d} s\right| \leq C\left(\|\operatorname{curl} \mathbf{u}\|_{L^{q}(T)}+\left\|\gamma_{\mathbf{t}} \mathbf{u}\right\|_{L^{q}(F)}\right)
$$

with $C=C(q, T)>0$. As in Lemma 3.13, $F$ has to be a face of $T$ adjacent to $e$, and $\mathbf{t}_{e}$ stands for a unit vector in the direction of $e$. A straightforward inspection of the construction given in Figure 3.5, the above stabilities of liftings and projections, and embeddings and standard trace theorems for Sobolev spaces thus reward us with the continuity

$$
\left\|\Pi_{p \mid T}^{1} \mathbf{u}\right\|_{L^{2}(T)} \leq C\left(\|\mathbf{u}\|_{\boldsymbol{H}^{\frac{1}{2}+s}(T)}+\|\operatorname{curl} \mathbf{u}\|_{\boldsymbol{H}^{s}(T)}\right),
$$

with $C=C(s, T)>0$ independent of $p$ and for any $s>0$.

Theorem 3.18. ( $p$-interpolation for discrete 1-forms) Under the assumptions made above, the projection-based interpolation operators $\Pi_{p}^{1}$, $p \in \mathbb{N}_{0}$, satisfy

$$
\left\|\mathbf{u}-\Pi_{p}^{1} \mathbf{u}\right\|_{\boldsymbol{L}^{2}(\Omega)} \leq C p^{\frac{1}{2}-\epsilon}\left(\|\mathbf{u}\|_{\boldsymbol{H}^{1}(\Omega)}+\|\operatorname{curl} \mathbf{u}\|_{\boldsymbol{H}^{1}(\Omega)}\right),
$$

for any $\epsilon>0$, and for all sufficiently smooth vector-fields $\mathbf{u}$ satisfying $C=$ $C\left(\rho\left(\Omega_{h}\right), \epsilon\right)>0$ independent of $p$. For all $r>1$ and $\epsilon>0$ we have, with $C=C\left(r, \epsilon, \rho\left(\Omega_{h}\right)\right)>0$,

$$
\left\|\mathbf{u}-\Pi_{p}^{1} \mathbf{u}\right\|_{\boldsymbol{L}^{2}(\Omega)} \leq C p^{r-1-\epsilon}\|\mathbf{u}\|_{\boldsymbol{H}^{r}(\Omega)}
$$

Proof. Thanks to scaling arguments it suffices to consider a single element $T$. Pick $\mathbf{u} \in \boldsymbol{H}^{1}(T)$, curl $\mathbf{u} \in \boldsymbol{H}^{1}(T)$, and use the decomposition of Lemma 2.6

$$
\mathbf{u}=\boldsymbol{\Psi}+\operatorname{grad} \varphi, \quad \boldsymbol{\Psi} \in \boldsymbol{H}^{2}(T), \varphi \in H^{2}(T) .
$$

Since $\varphi$ is continuous on $T, \Pi_{p}^{0} \varphi$ is well defined. The same holds true for $\Pi_{p}^{1} \Psi$ and we can use the commuting diagram property in order to get

$$
\left\|\mathbf{u}-\Pi_{p}^{1} \mathbf{u}\right\|_{\boldsymbol{L}^{2}(T)} \leq\left\|\boldsymbol{\Psi}-\Pi_{p}^{1} \boldsymbol{\Psi}\right\|_{\boldsymbol{L}^{2}(T)}+\left|\varphi-\Pi_{p}^{0} \varphi\right|_{H^{1}(T)} .
$$

The interpolation operators $\Pi_{p}^{1}$ and $\Pi_{p}^{0}$ locally preserve polynomials of degree $p$ and $p+1$, respectively. Thus,

$$
\begin{aligned}
\left\|\mathbf{u}-\Pi_{p}^{1} \mathbf{u}\right\|_{\boldsymbol{L}^{2}(T)} \leq & \inf _{\mathbf{p} \in\left(\mathcal{P}_{p}(T)\right)^{3}}\left\|\left(\operatorname{Id}-\Pi_{p}^{1}\right)(\mathbf{\Psi}-\mathbf{p})\right\|_{\boldsymbol{L}^{2}(T)} \\
& +\inf _{p \in \mathcal{P}_{p+1}(T)}\left|\left(\operatorname{Id}-\Pi_{p}^{0}\right)(\varphi-p)\right|_{H^{1}(T)} .
\end{aligned}
$$


The $p$-uniform continuity of the interpolation operators leads to

$$
\begin{aligned}
& l\left\|\mathbf{u}-\Pi_{p}^{1} \mathbf{u}\right\|_{\boldsymbol{L}^{2}(T)} \leq \\
& \quad C\left(\inf _{\mathbf{p} \in\left(\mathcal{P}_{p}(T)\right)^{3}}\|\boldsymbol{\Psi}-\mathbf{p}\|_{\boldsymbol{H}^{1+\epsilon}(T)}+\inf _{p \in \mathcal{P}_{p+1}(T)}\|\varphi-p\|_{H^{\frac{3}{2}+\epsilon}(T)}\right),
\end{aligned}
$$

for any $\epsilon>0$ and with $C=C(\epsilon, T)>0$. Standard estimates for best approximation by polynomials (Braess and Schwab 2000, Theorem 3.3) and the stability of the splitting yield the first assertion. Using the continuity

$$
\left\|\Pi_{p \mid T}^{1} \mathbf{u}\right\|_{\boldsymbol{L}^{2}(T)} \leq C\|\mathbf{u}\|_{\boldsymbol{H}^{1+s}(T)} \quad \forall \mathbf{u} \in \boldsymbol{H}^{1+s}(T),
$$

the proof of the second only involves the last two steps in the above considerations.

\section{Bibliographical notes}

Estimates for a projection-based interpolation operator for discrete 1-forms in 2D are given in Demkowicz and Babuška (2001). Approximation estimates for higher-order discrete 1-forms on quadrilaterals and hexahedra are covered in Monk (1994) and Ainsworth and Pinchedez (2001).

\section{Maxwell eigenvalue problem}

In our first model problem $\Omega \subset A\left(\mathbb{R}^{3}\right)$ plays the role of a bounded cavity filled with non-conducting dielectric material and lined by ideally conducting walls. The material parameters $\boldsymbol{\epsilon}, \boldsymbol{\mu}$ are supposed to be piecewise constant. We aim to compute resonant frequencies and related eigenmodes of $\Omega$, that is, both $\omega \neq 0$ and electromagnetic fields have to be determined, such that Maxwell's equations (2.1), (2.2) and $\gamma_{\mathbf{t}} \mathbf{e}=0$ on $\partial \Omega$ are satisfied. This task is called the (electric) Maxwell eigenvalue problem.

Following the reasoning in Section 2.3, primal and dual variational formulations of the Maxwell eigenvalue problem can be derived. In terms of vector proxies the primal, e-based formulation reads: Find $\mathbf{e} \in \boldsymbol{H}_{0}(\mathbf{c u r l} ; \Omega), \omega \neq 0$ such that

$$
\left(\boldsymbol{\mu}^{-1} \operatorname{curl} \mathbf{e}, \operatorname{curl} \mathbf{e}^{\prime}\right)_{\boldsymbol{L}^{2}(\Omega)}=\omega^{2}\left(\boldsymbol{\epsilon} \mathbf{e}, \mathbf{e}^{\prime}\right)_{\boldsymbol{L}^{2}(\Omega)} \quad \forall \mathbf{e}^{\prime} \in \boldsymbol{H}_{0}(\operatorname{curl} ; \Omega) .
$$

The dual, h-based formulation seeks $\mathbf{h} \in \boldsymbol{H}(\mathbf{c u r l} ; \Omega), \omega \neq 0$, such that

$$
\left(\boldsymbol{\epsilon}^{-1} \operatorname{curl} \mathbf{h}, \operatorname{curl} \mathbf{h}^{\prime}\right)_{\boldsymbol{L}^{2}(\Omega)}=\omega^{2}\left(\boldsymbol{\mu} \mathbf{h}, \mathbf{h}^{\prime}\right)_{\boldsymbol{L}^{2}(\Omega)} \quad \forall \mathbf{h}^{\prime} \in \boldsymbol{H}(\operatorname{curl} ; \Omega) .
$$

The treatment of (4.1) and (4.2) is very similar. In the following we restrict our attention to (4.1). 


\subsection{Embeddings}

As far as structure is concerned, the eigenvalue problem (4.1) much resembles the Dirichlet eigenvalue problem for the Laplacian. However, the infinite-dimensional kernel of curl introduces a pronounced difference: In contrast to $\Delta$ the operator curl curl fails to have a compact resolvent in $\boldsymbol{L}^{2}(\Omega)$. A fundamental prerequisite for the application of the powerful RieszSchauder spectral theory for compact operators is missing.

Yet, as can be seen by testing with irrotational functions, due to $\omega \neq 0$, (4.1) can be equivalently stated on the space

$$
\boldsymbol{Z}_{0}(\boldsymbol{\epsilon}, \Omega):=\left\{\mathbf{u} \in \boldsymbol{H}_{0}(\operatorname{curl} ; \Omega),(\boldsymbol{\epsilon} \mathbf{u}, \mathbf{z})_{\boldsymbol{L}^{2}(\Omega)}=0 \forall \mathbf{z} \in \boldsymbol{H}_{0}(\operatorname{curl} 0 ; \Omega)\right\} \text {. }
$$

Seek $\mathbf{e} \in \boldsymbol{Z}_{0}(\boldsymbol{\epsilon}, \Omega), \omega \neq 0$ such that

$$
\left(\mu^{-1} \operatorname{curl} \mathbf{e}, \operatorname{curl} \mathbf{e}^{\prime}\right)_{\boldsymbol{L}^{2}(\Omega)}=\omega^{2}\left(\boldsymbol{\epsilon} \mathbf{e}, \mathbf{e}^{\prime}\right)_{\boldsymbol{L}^{2}(\Omega)} \quad \forall \mathbf{e}^{\prime} \in \boldsymbol{Z}_{0}(\boldsymbol{\epsilon}, \Omega)
$$

The advantage of the formulation (4.4) is clear from the following fundamental embedding result. To state it, we let $\boldsymbol{\alpha}$ denote a generic piecewise smooth metric tensor and introduce the Hilbert space

$$
\boldsymbol{X}_{0}(\boldsymbol{\alpha}, \Omega):=\left\{\mathbf{u} \in \boldsymbol{H}_{0}(\mathbf{c u r l} ; \Omega), \operatorname{div}(\boldsymbol{\alpha} \mathbf{u}) \in L^{2}(\Omega)\right\},
$$

equipped with the natural graph norm. It is essential that the extra constraint on the divergence involved in the definition of $\boldsymbol{X}_{0}(\boldsymbol{\alpha}, \Omega)$ enforces a slightly enhanced regularity of the vector-fields ( $c f$. Amrouche et al. (1998, Proposition 3.7)).

Theorem 4.1. There exists $s_{0}>0$ such that the space $\boldsymbol{X}_{0}(\boldsymbol{\alpha}, \Omega)$ is continuously embedded in $\boldsymbol{H}^{s}(\Omega)$ for all $s<s_{0}$. If $\boldsymbol{\alpha}$ is uniformly Lipschitzcontinuous, we can choose $s_{0}>\frac{1}{2}$.

Proof. Pick any $\mathbf{u} \in \boldsymbol{X}_{0}(\boldsymbol{\alpha}, \Omega)$ and use the stable regular decomposition from Lemma 2.4:

$$
\mathbf{u}=\boldsymbol{\Psi}+\operatorname{grad} \varphi, \quad \boldsymbol{\Psi} \in \boldsymbol{H}^{1}(\Omega) \cap \boldsymbol{H}_{0}(\operatorname{curl} ; \Omega), \varphi \in H_{0}^{1}(\Omega) .
$$

Formally, we find that $\varphi$ satisfies

$$
\varphi \in H_{0}^{1}(\Omega): \quad-\operatorname{div}(\boldsymbol{\alpha} \operatorname{grad} \varphi)=\operatorname{div}(\boldsymbol{\alpha} \boldsymbol{\Psi})-\operatorname{div}(\boldsymbol{\alpha} \mathbf{u}) .
$$

Since $\boldsymbol{\alpha}$ is piecewise smooth and $\boldsymbol{\Psi} \in \boldsymbol{H}^{1}(\Omega)$, we conclude that $\operatorname{div}(\boldsymbol{\alpha} \boldsymbol{\Psi}) \in$ $H^{-\frac{1}{2}-\epsilon}(\Omega)$ for any $\epsilon>0$.

Now we invoke lifting theorems for the Dirichlet problem for second-order elliptic operators on curvilinear Lipschitz polyhedra, and in the case of piecewise smooth coefficients. These can be found in Dauge (1988) and Costabel et al. (1999, Section 4). They guarantee the existence of $s_{\text {Dir }}^{*}(\boldsymbol{\alpha})>1$ such that for all $1 \leq t<s_{\text {Dir }}^{*}(\boldsymbol{\alpha})$, the solution of

$$
\phi \in H_{0}^{1}(\Omega): \quad \operatorname{div}(\boldsymbol{\alpha} \operatorname{grad} \phi)=f \in H^{t-2}(\Omega) \text { in } \Omega
$$


satisfies $\varphi \in H^{t}(\Omega)$ and $\|\varphi\|_{H^{t}(\Omega)} \leq C\|f\|_{H^{t-2}(\Omega)}$. This sophisticated result is obtained by studying corner, edge and interface singularities of solutions of the Dirichlet problem for the Laplacian. Thus, we learn that $\varphi \in H^{t}(\Omega)$ for all $t<\min \left\{s_{\text {Dir }}^{*}(\boldsymbol{\alpha}), \frac{3}{2}\right\}$, which involves the first assertion of the theorem with $s_{0}:=\min \left\{s_{\text {Dir }}^{*}(\boldsymbol{\alpha})-1, \frac{1}{2}\right\}$.

To get the second, remember that it is known from the work of Dauge (1988, Chapter 6) (see also Costabel and Dauge (1998)) that $s_{\text {Dir }}^{*}(\mathbf{I})>\frac{3}{2}$. Further, note that for uniformly Lipschitz-continuous $\boldsymbol{\alpha}$ the right-hand side in (4.6) belongs to $L^{2}(\Omega)$.

The same technique enables us to establish another embedding theorem (cf. Amrouche et al. (1998, Proposition 3.7) and Hazard and Lenoir (1996, Lemma B8)).

Lemma 4.2. If $\mathbf{u} \in \boldsymbol{H}(\operatorname{curl} ; \Omega), \operatorname{div}(\boldsymbol{\alpha u})=0$, and $\gamma_{\mathbf{n}}(\boldsymbol{\alpha u})=0$ on $\partial \Omega$, then there exists $r_{0}>0$ such that $\mathbf{u} \in \boldsymbol{H}^{r}(\Omega)$ for all $0<r<r_{0}$ and

$$
\|\mathbf{u}\|_{\boldsymbol{H}^{r}(\Omega)} \leq C\left(\|\mathbf{u}\|_{\boldsymbol{L}^{2}(\Omega)}+\|\operatorname{curl} \mathbf{u}\|_{\boldsymbol{L}^{2}(\Omega)}+\|\operatorname{div}(\boldsymbol{\alpha} \mathbf{u})\|_{L^{2}(\Omega)}\right),
$$

with $C=C(\Omega, r)>0$. If $\boldsymbol{\alpha}$ is uniformly Lipschitz-continuous, then we can choose $r_{0}>\frac{1}{2}$.

Recalling Rellich's theorem for scales of classical Sobolev space we can infer a compact embedding ( $c f$. Amrouche et al. (1998, Theorem 2.8) and Jochmann (1997)).

Corollary 4.3. The embedding $\boldsymbol{X}_{0}(\boldsymbol{\epsilon}, \Omega) \hookrightarrow \boldsymbol{L}^{2}(\Omega)$ is compact.

This compact embedding immediately implies a Poincaré-Friedrichs-type inequality, because $\operatorname{div}(\boldsymbol{\epsilon} \mathbf{u})=0$ for all $\mathbf{u} \in \boldsymbol{Z}_{0}(\boldsymbol{\epsilon}, \Omega)$.

Corollary 4.4. (Poincaré-Friedrichs-type inequality) There is a constant $C>0$ depending on $\Omega$ only, such that

$$
\|\mathbf{u}\|_{\boldsymbol{L}^{2}(\Omega)} \leq C\|\operatorname{curl} \mathbf{u}\|_{\boldsymbol{L}^{2}(\Omega)} \quad \forall \mathbf{u} \in \boldsymbol{Z}_{0}(\boldsymbol{\epsilon}, \Omega) .
$$

Therefore, by the Lax-Milgram lemma, the variational equation

$$
\left(\boldsymbol{\mu}^{-1} \operatorname{curl} \mathrm{Tu}, \operatorname{curl} \mathbf{u}^{\prime}\right)_{\boldsymbol{L}^{2}(\Omega)}=\left(\boldsymbol{\epsilon} \mathbf{u}, \mathbf{u}^{\prime}\right)_{L^{2}(\Omega)} \quad \forall \mathbf{u}^{\prime} \in \boldsymbol{Z}_{0}(\boldsymbol{\epsilon}, \Omega),
$$

defines a continuous operator $\mathrm{T}: \boldsymbol{L}^{2}(\Omega) \mapsto \boldsymbol{Z}_{0}(\boldsymbol{\epsilon}, \Omega)$, which is compact as an operator $\mathrm{T}: \boldsymbol{L}^{2}(\Omega) \mapsto \boldsymbol{L}^{2}(\Omega)$. As such, $\mathrm{T}$ is also self-adjoint by virtue of the symmetry of the bilinear forms involved in its definition.

By means of $\mathrm{T}$ the eigenvalue problem (4.4) can be recast as

$$
\left(\boldsymbol{\mu}^{-1} \operatorname{curle}, \operatorname{curl} \mathbf{e}^{\prime}\right)_{\boldsymbol{L}^{2}(\Omega)}=\omega^{2}\left(\boldsymbol{\mu}^{-1} \operatorname{curl} \mathrm{Te}, \operatorname{curl} \mathbf{e}^{\prime}\right)_{\boldsymbol{L}^{2}(\Omega)} \forall \mathbf{e}^{\prime} \in \boldsymbol{Z}_{0}(\boldsymbol{\epsilon}, \Omega),
$$

and converted into the operator eigenvalue problem

$$
\mathrm{Te}=\omega^{-2} \mathbf{e}
$$


Thus, the Riesz-Schauder theory for the spectrum of compact, self-adjoint operators in Hilbert space implies that (4.1) has an increasing sequence $0<\omega_{1}<\omega_{2}<\cdots$ of nonzero real 'Maxwell eigenvalues' tending to $\infty$. They all have finite multiplicity and the corresponding eigenspaces are mutually $\boldsymbol{L}^{2}(\Omega)$-orthogonal. Hence, by switching to the complement $\boldsymbol{Z}_{0}(\boldsymbol{\epsilon}, \Omega)$ of $\operatorname{Ker}(\mathbf{c u r l})$ we have recovered a situation typical of second-order elliptic eigenproblems. We point out that $\omega_{k} \in \mathbb{R}$ makes it possible to work with real field amplitudes only, when solving a Maxwell eigenvalue problem.

\section{Bibliographical notes}

The main reference for this section is Amrouche et al. (1998, Sections 2,3). The statement of Corollary 4.3 was first proved in Weber (1980) and can also be found in Picard (1984), and an extension to mixed boundary conditions can be found in Jochmann (1997).

\subsection{Fortin projectors}

Using the space $\mathcal{W}_{p, 0}^{1}\left(\Omega_{h}\right):=\boldsymbol{H}_{0}(\operatorname{curl} ; \Omega) \cap \mathcal{W}_{p}^{1}\left(\Omega_{h}\right)$ of discrete 1 -forms on a triangulation $\Omega_{h}$ of $\Omega$ a Galerkin discretization of (4.1) is straightforward. Seek $\mathbf{e}_{h} \in \mathcal{W}_{p, 0}^{1}\left(\Omega_{h}\right), \omega_{h} \neq 0$ such that

$$
\left(\boldsymbol{\mu}^{-1} \operatorname{curl} \mathbf{e}_{h}, \operatorname{curl} \mathbf{e}_{h}^{\prime}\right)_{\boldsymbol{L}^{2}(\Omega)}=\omega_{h}^{2}\left(\boldsymbol{\epsilon} \mathbf{e}_{h}, \mathbf{e}_{h}^{\prime}\right)_{\boldsymbol{L}^{2}(\Omega)} \quad \forall \mathbf{e}_{h}^{\prime} \in \mathcal{W}_{p, 0}^{1}\left(\Omega_{h}\right) .
$$

Below we examine the convergence of $\omega_{h}$ and $\mathbf{u}_{h}$ for the $h$-version of discrete differential forms. Throughout we take for granted affine equivalence of the spaces $\mathcal{W}_{p, 0}^{1}\left(\Omega_{h}\right)$ and $\mathcal{W}_{p, 0}^{2}\left(\Omega_{h}\right)$ of discrete forms as well as the commuting diagram property for the moment-based local projectors $\Pi_{p}^{1}$ and $\Pi_{p}^{2}$.

Parallel to the continuous case, a variational problem equivalent to (4.7) can be posed on the space

$$
\begin{aligned}
& \boldsymbol{Z}_{h, 0}\left(\boldsymbol{\epsilon}, \Omega_{h}\right)= \\
& \left\{\mathbf{u}_{h} \in \mathcal{W}_{p, 0}^{1}\left(\Omega_{h}\right),\left(\boldsymbol{\epsilon} \mathbf{u}_{h}, \mathbf{z}_{h}\right)_{\boldsymbol{L}^{2}(\Omega)}=0 \forall \mathbf{z}_{h} \in \mathcal{W}_{p, 0}^{1}\left(\Omega_{h}\right) \cap \operatorname{Ker}(\mathbf{c u r l})\right\} .
\end{aligned}
$$

Does this pave the way for studying the approximation of Maxwell eigenvalues along the same lines as for the discrete Laplacian, namely by appealing to the theory of eigenvalue approximation for self-adjoint positive definite operators with compact resolvent? Unfortunately, this hope is dashed by the observation that, in general,

$$
\boldsymbol{Z}_{h, 0}\left(\boldsymbol{\epsilon}, \Omega_{h}\right) \not \subset \boldsymbol{Z}_{0}(\boldsymbol{\epsilon}, \Omega) \text {. }
$$

Bluntly speaking, in terms of (4.4) the variational problem (4.7) when restricted to $\boldsymbol{Z}_{h, 0}\left(\boldsymbol{\epsilon}, \Omega_{h}\right)$ is a non-conforming discretization. What is available in this case is the theory of spectral approximation of compact operators from Babuška and Osborn (1991). It requires us to introduce a discrete operator $\mathrm{T}_{h}: \boldsymbol{L}^{2}(\Omega) \mapsto \boldsymbol{Z}_{h, 0}\left(\boldsymbol{\epsilon}, \Omega_{h}\right)$ that approximates $\mathrm{T}$. To begin with, we 


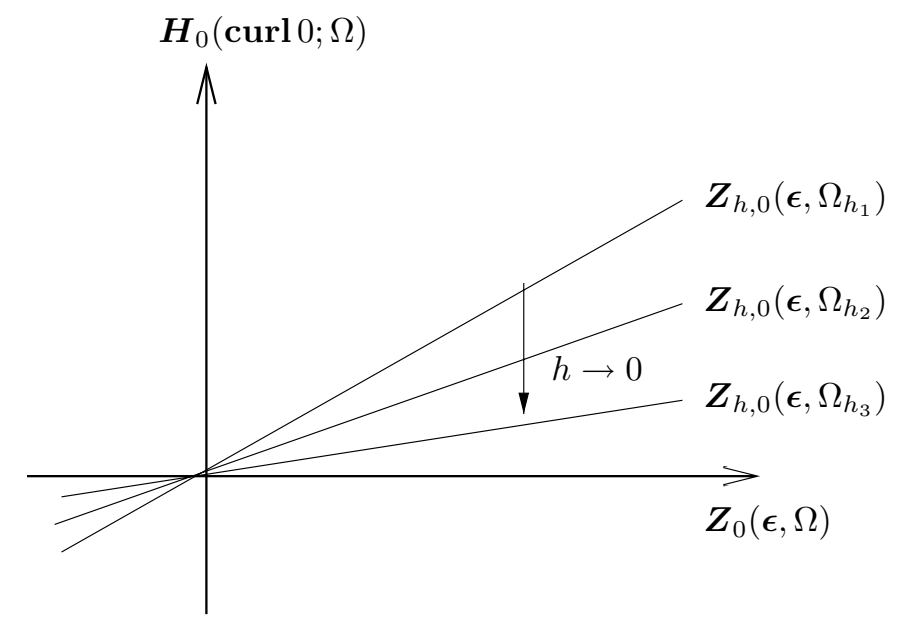

Figure 4.1. Graphical illustration of the message of Lemma 4.5. The plane of the paper represents $\boldsymbol{H}(\mathbf{c u r l} ; \Omega)$ with its natural geometry

badly need a link between $\boldsymbol{Z}_{0}(\boldsymbol{\epsilon}, \Omega)$ and its non-conforming approximating space $\boldsymbol{Z}_{h, 0}\left(\boldsymbol{\epsilon}, \Omega_{h}\right)$. It is provided by the Hodge mapping $\boldsymbol{H}_{\boldsymbol{\epsilon}}: \boldsymbol{H}_{0}(\mathbf{c u r l} ; \Omega) \mapsto$ $\boldsymbol{Z}_{0}(\boldsymbol{\epsilon}, \Omega)$, an $(\boldsymbol{\epsilon} \cdot, \cdot)_{\boldsymbol{L}^{2}(\Omega)}$-orthogonal projection, defined by

$$
\mathrm{H}_{\boldsymbol{\epsilon}} \mathbf{u} \in \boldsymbol{Z}_{0}(\boldsymbol{\epsilon}, \Omega): \quad \operatorname{curl}_{\boldsymbol{\epsilon}} \mathbf{u}=\operatorname{curl} \mathbf{u}, \quad \mathbf{u} \in \boldsymbol{H}_{0}(\operatorname{curl} ; \Omega)
$$

Thanks to Corollary 4.4 this is a valid definition, because an element of $\boldsymbol{Z}_{0}(\boldsymbol{\epsilon}, \Omega)$ is already uniquely determined by its curl. By the following approximation result we see that $\boldsymbol{Z}_{0}(\boldsymbol{\epsilon}, \Omega)$ and $\boldsymbol{Z}_{h, 0}\left(\boldsymbol{\epsilon}, \Omega_{h}\right)$ are close on fine meshes, provided that $\epsilon$ is regular enough: the gap between them will shrink to zero as $h \rightarrow 0$, as illustrated in Figure 4.1.

Lemma 4.5. (Approximation estimate for the Hodge map on $\mathcal{Z}_{h, 0}$ ) If $\boldsymbol{\epsilon}$ is uniformly Lipschitz-continuous, we have

$$
\left\|\mathbf{u}_{h}-\mathrm{H}_{\boldsymbol{\epsilon}} \mathbf{u}_{h}\right\|_{\boldsymbol{L}^{2}(\Omega)} \leq C h^{s}\left\|\operatorname{curl} \mathbf{u}_{h}\right\|_{\boldsymbol{L}^{2}(\Omega)} \quad \forall \mathbf{u}_{h} \in \boldsymbol{Z}_{h, 0}\left(\boldsymbol{\epsilon}, \Omega_{h}\right),
$$

for $\frac{1}{2}<s<s_{0}, s_{0}$ from Theorem 4.1, and a constant $C>0$ depending only on $s, \epsilon, \Omega$, and the shape regularity of $\Omega_{h}$.

The proof will make use of the following technical result.

Lemma 4.6. Let $\frac{1}{2}<s \leq 1$, and $\mathbf{u} \in \boldsymbol{H}^{s}(\Omega) \cap \boldsymbol{H}_{0}(\operatorname{curl} ; \Omega)$. If curl u belongs to the space $\mathcal{W}_{p, 0}^{2}\left(\Omega_{h}\right)$ of discrete 2 -forms on $\Omega_{h}$, then

$$
\left\|\mathbf{u}-\Pi_{p}^{1} \mathbf{u}\right\|_{\boldsymbol{L}^{2}(\Omega)} \leq C h^{s}|\mathbf{u}|_{\boldsymbol{H}^{s}(\Omega)},
$$

with $C=C\left(\Omega, s, p, \rho\left(\Omega_{h}\right)\right)>0$ independent of $\mathbf{u}$ and $h$. 
Proof. The idea of the proof can be found in Amrouche et al. (1998, Section 4). Consider a single element $T \in \mathcal{S}_{3}\left(\Omega_{h}\right)$. We conclude from Lemma 3.13 and trace/embedding theorems for Sobolev spaces that the moment-based local interpolant $\Pi_{p_{\mid T}}^{l} \mathbf{u}$ is well defined, since $\gamma_{\mathbf{t}} \mathbf{u} \in \boldsymbol{H}^{s-\frac{1}{2}}(F)$ for each face $F$ of $T$ and curl $\mathbf{u}_{\mid T}$ is smooth.

Then use the transformation formulae of Lemma 3.12 to get, with $\widehat{\mathbf{u}}:=$ $\mathfrak{F}_{\boldsymbol{\Phi}_{T}}^{1} \mathbf{u}$

$$
\begin{aligned}
\left\|\mathbf{u}-\Pi_{p}^{1} \mathbf{u}\right\|_{\boldsymbol{L}^{2}(T)} & \leq C h_{T}^{\frac{1}{2}}\left\|\left(\operatorname{Id}-\widehat{\Pi_{p}^{1}}\right) \widehat{\mathbf{u}}\right\|_{\boldsymbol{L}^{2}(\widehat{T})} \\
& \leq C h_{T}^{\frac{1}{2}} \inf _{\mathbf{p} \in\left(\mathcal{P}_{p}(\widehat{T})\right)^{3}}\left(\|\widehat{\mathbf{u}}-\mathbf{p}\|_{\boldsymbol{H}^{s}(\widehat{T})}+\|\mathbf{c u r l}(\widehat{\mathbf{u}}-\mathbf{p})\|_{\boldsymbol{H}^{s-\frac{1}{2}}(\widehat{T})}\right) .
\end{aligned}
$$

Observe that $\operatorname{curl}(\widehat{\mathbf{u}}-\mathbf{p})$ belongs to $\mathcal{W}_{p}^{2}(\widehat{T})$. All norms on the finitedimensional space $\operatorname{curl} \mathcal{W}_{p}^{1}(\widehat{T}) \subset \mathcal{W}_{p}^{2}(\widehat{T})$ are equivalent, whence

$$
\|\operatorname{curl}(\widehat{\mathbf{u}}-\mathbf{p})\|_{\boldsymbol{H}^{s-\frac{1}{2}}(\widehat{T})} \leq C\|\widehat{\mathbf{u}}-\mathbf{p}\|_{\boldsymbol{H}^{s}(\widehat{T})} .
$$

This permits us to continue the estimate by

$$
\begin{aligned}
& \left\|\mathbf{u}-\Pi_{p}^{1} \mathbf{u}\right\|_{\boldsymbol{L}^{2}(T)} \leq \\
& \quad C h_{T}^{\frac{1}{2}} \inf _{\mathbf{p} \in\left(\mathcal{P}_{p}(\widehat{T})\right)^{3}}\|\widehat{\mathbf{u}}-\mathbf{p}\|_{\boldsymbol{H}^{s}(\widehat{T})} \leq C h_{T}^{\frac{1}{2}}|\widehat{\mathbf{u}}|_{\boldsymbol{H}^{s}(\widehat{T})} \leq C h_{T}^{s}\|\mathbf{u}\|_{\boldsymbol{H}^{s}(T)},
\end{aligned}
$$

where we used the Bramble-Hilbert lemma for fractional Sobolev norms on $\widehat{T}$ (Dupont and Scott 1980, Proposition 6.1) and Lemma 3.12. The generic constants $C>0$ only depend on $s, p$, and the shape regularity measure $\rho\left(\Omega_{h}\right)$. Summing this estimate over all elements yields the assertion.

Proof of Lemma 4.5. Pick any $\mathbf{u}_{h} \in \boldsymbol{Z}_{h, 0}\left(\boldsymbol{\epsilon}, \Omega_{h}\right)$. As $\mathrm{H}_{\boldsymbol{\epsilon}} \mathbf{u}_{h} \in \boldsymbol{Z}_{0}(\boldsymbol{\epsilon}, \Omega)$, we know from Theorem 4.1 ( $\boldsymbol{\epsilon}$ uniformly Lipschitz-continuous) that

$$
\left\|\mathrm{H}_{\boldsymbol{\epsilon}} \mathbf{u}_{h}\right\|_{\boldsymbol{H}^{s}(\Omega)} \leq C\left(\left\|\mathrm{H}_{\boldsymbol{\epsilon}} \mathbf{u}_{h}\right\|_{\boldsymbol{L}^{2}(\Omega)}+\left\|\operatorname{curl} \mathrm{H}_{\boldsymbol{\epsilon}} \mathbf{u}_{h}\right\|_{\boldsymbol{L}^{2}(\Omega)}+\left\|\operatorname{div}\left(\boldsymbol{\epsilon} \mathrm{H}_{\boldsymbol{\epsilon}} \mathbf{u}_{h}\right)\right\|_{L^{2}(\Omega)}\right),
$$

with $\frac{1}{2}<s<s_{0}$. By definition of $\mathrm{H}_{\boldsymbol{\epsilon}}$, Theorem 4.1, and the previous lemma we learn that $\Pi_{p}^{1} \mathrm{H}_{\boldsymbol{\epsilon}} \mathbf{u}_{h}$ is well defined. This permits us to apply Nédélec's trick, from Nédélec (1980),

$$
\begin{aligned}
& \left\|\boldsymbol{\epsilon}^{\frac{1}{2}}\left(\mathbf{u}_{h}-\mathrm{H}_{\boldsymbol{\epsilon}} \mathbf{u}_{h}\right)\right\|_{\boldsymbol{L}^{2}(\Omega)}^{2} \\
& \quad=\left(\boldsymbol{\epsilon}\left(\mathbf{u}_{h}-\mathrm{H}_{\boldsymbol{\epsilon}} \mathbf{u}_{h}\right), \mathbf{u}_{h}-\Pi_{p}^{1} \mathrm{H}_{\boldsymbol{\epsilon}} \mathbf{u}_{h}+\Pi_{p}^{1} \mathrm{H}_{\boldsymbol{\epsilon}} \mathbf{u}_{h}-\mathrm{H}_{\boldsymbol{\epsilon}} \mathbf{u}_{h}\right) \boldsymbol{L}^{2}(\Omega) \\
& \quad=\left(\boldsymbol{\epsilon}\left(\mathbf{u}_{h}-\mathrm{H}_{\boldsymbol{\epsilon}} \mathbf{u}_{h}\right), \Pi_{p}^{1} \mathrm{H}_{\boldsymbol{\epsilon}} \mathbf{u}_{h}-\mathrm{H}_{\boldsymbol{\epsilon}} \mathbf{u}_{h}\right) \boldsymbol{L}^{2}(\Omega) \\
& \quad \leq\left\|\boldsymbol{\epsilon}^{\frac{1}{2}}\left(\mathbf{u}_{h}-\mathrm{H}_{\boldsymbol{\epsilon}} \mathbf{u}_{h}\right)\right\|_{\boldsymbol{L}^{2}(\Omega)}\left\|\boldsymbol{\epsilon}^{\frac{1}{2}}\left(\Pi_{p}^{1}-\mathrm{Id}\right) \mathrm{H}_{\boldsymbol{\epsilon}} \mathbf{u}_{h}\right\|_{\boldsymbol{L}^{2}(\Omega)} .
\end{aligned}
$$


The commuting diagram property is pivotal here. It guarantees that

$$
\operatorname{curl}\left(\mathbf{u}_{h}-\Pi_{p}^{1} \mathrm{H}_{\boldsymbol{\epsilon}} \mathbf{u}_{h}\right)=\operatorname{curl}\left(\Pi_{p}^{1}\left(\mathbf{u}_{h}-\mathrm{H}_{\boldsymbol{\epsilon}} \mathbf{u}_{h}\right)\right)=\Pi_{p}^{2}\left(\operatorname{curl}\left(\mathbf{u}_{h}-\mathrm{H}_{\boldsymbol{\epsilon}} \mathbf{u}_{h}\right)\right)=0,
$$

by definition of $\boldsymbol{H}_{\boldsymbol{\epsilon}}$. Since both $\boldsymbol{Z}_{0}(\boldsymbol{\epsilon}, \Omega)$ and $\boldsymbol{Z}_{h, 0}\left(\boldsymbol{\epsilon}, \Omega_{h}\right)$ are $(\boldsymbol{\epsilon} \cdot, \cdot)_{\boldsymbol{L}^{2}(\Omega)^{-}}$ orthogonal to $\operatorname{Ker}(\mathbf{c u r l}) \cap \mathcal{W}_{p, 0}^{1}\left(\Omega_{h}\right)$ the above manipulations are justified. Then we can use the interpolation estimate of Lemma 4.5, $\operatorname{div}\left(\boldsymbol{\epsilon} \mathrm{H}_{\boldsymbol{\epsilon}} \mathbf{u}_{h}\right)=0$, and the fact that $\epsilon$ is uniformly bounded from above and below, and get from (4.9)

$$
\left\|\mathbf{u}_{h}-\mathrm{H}_{\boldsymbol{\epsilon}} \mathbf{u}_{h}\right\|_{\boldsymbol{L}^{2}(\Omega)} \leq C h^{s}\left\|\mathrm{H}_{\boldsymbol{\epsilon}} \mathbf{u}_{h}\right\|_{\boldsymbol{H}^{s}(\Omega)} \leq C h^{s}\left\|\operatorname{curl} \mathrm{H}_{\boldsymbol{\epsilon}} \mathbf{u}_{h}\right\|_{\boldsymbol{L}^{2}(\Omega)} .
$$

This amounts to the contention of the theorem.

Now we are in a position to establish a discrete version of the PoincaréFriedrichs inequality from Corollary 4.4.

Theorem 4.7. (Discrete Poincaré-Friedrichs inequality) There is a positive constant $C$ depending only on $\Omega, \boldsymbol{\epsilon}, p$ and the shape regularity of the mesh, such that

$$
\left\|\mathbf{u}_{h}\right\|_{\boldsymbol{L}^{2}(\Omega)} \leq C\left\|\operatorname{curl} \mathbf{u}_{h}\right\|_{\boldsymbol{L}^{2}(\Omega)} \quad \forall \mathbf{u}_{h} \in \boldsymbol{Z}_{h, 0}\left(\boldsymbol{\epsilon}, \Omega_{h}\right)
$$

Proof. Let I stand for the $3 \times 3$ identity matrix. Then, pick some $\mathbf{u}_{h} \in$ $\boldsymbol{Z}_{h, 0}\left(\boldsymbol{\epsilon}, \Omega_{h}\right)$ and use its Helmholtz decompositions

$$
\mathbf{u}_{h}=\left\{\begin{array}{cl}
\mathbf{z}+\mathbf{q}, & \mathbf{z} \in \boldsymbol{Z}_{0}(\mathbf{I}, \Omega), \operatorname{curl} \mathbf{q}=0 \\
\mathbf{z}_{h}+\mathbf{q}_{h}, & \mathbf{z}_{h} \in \boldsymbol{Z}_{h, 0}\left(\mathbf{I}, \Omega_{h}\right), \operatorname{curl} \mathbf{q}_{h}=0
\end{array}\right.
$$

From the definition of $\mathbf{H}_{\mathbf{I}}$ it is clear that $\mathbf{z}=\mathrm{H}_{\mathbf{I}} \mathbf{z}_{h}$. Hence, by Lemma 4.5 we find $s>\frac{1}{2}$ and $C=C\left(\Omega, \rho\left(\Omega_{h}\right)\right)>0$ such that

$$
\left\|\mathbf{z}-\mathbf{z}_{h}\right\|_{\boldsymbol{L}^{2}(\Omega)} \leq C h^{s}\left\|\operatorname{curl} \mathbf{u}_{h}\right\|_{\boldsymbol{L}^{2}(\Omega)} .
$$

We can exploit the orthogonality in the definition of $\boldsymbol{Z}_{h, 0}\left(\boldsymbol{\epsilon}, \Omega_{h}\right)$ and get

$$
\begin{aligned}
\left(\boldsymbol{\epsilon} \mathbf{u}_{h}, \mathbf{u}_{h}\right)_{\boldsymbol{L}^{2}(\Omega)} & =\left(\boldsymbol{\epsilon} \mathbf{u}_{h}, \mathbf{z}_{h}+\mathbf{q}_{h}\right)_{\boldsymbol{L}^{2}(\Omega)}=\left(\boldsymbol{\epsilon} \mathbf{u}_{h}, \mathbf{z}_{h}\right)_{\boldsymbol{L}^{2}(\Omega)} \\
& =\left(\boldsymbol{\epsilon} \mathbf{u}_{h}, \mathbf{z}_{h}-\mathbf{z}+\mathbf{z}\right) \boldsymbol{L}^{2}(\Omega) \\
& \leq C\left(\boldsymbol{\epsilon} \mathbf{u}_{h}, \mathbf{u}_{h}\right)_{\boldsymbol{L}^{2}(\Omega)}^{\frac{1}{2}}\left(\left\|\mathbf{z}-\mathbf{z}_{h}\right\|_{\boldsymbol{L}^{2}(\Omega)}+\|\mathbf{z}\|_{\boldsymbol{L}^{2}(\Omega)}\right)
\end{aligned}
$$

with $C>0$ depending on $\boldsymbol{\epsilon}$. Assembling the estimates and using Corollary 4.4 yields the assertion.

The Hodge mapping takes us from $\boldsymbol{Z}_{h, 0}\left(\boldsymbol{\epsilon}, \Omega_{h}\right)$ to $\boldsymbol{Z}_{0}(\boldsymbol{\epsilon}, \Omega)$. The opposite direction is covered by the Fortin projector $\mathrm{F}_{h}: \boldsymbol{H}_{0}(\operatorname{curl} ; \Omega) \mapsto \boldsymbol{Z}_{h, 0}\left(\boldsymbol{\epsilon}, \Omega_{h}\right)$, a term that originated in the theory of mixed finite elements (Brezzi and 
Fortin 1991, Section II.2.3). Due to the discrete Poincaré-Friedrichs inequality, $\mathbf{F}_{h} \mathbf{u} \in \boldsymbol{Z}_{h, 0}\left(\boldsymbol{\epsilon}, \Omega_{h}\right)$ can be defined for all $\mathbf{u} \in \boldsymbol{H}_{0}(\mathbf{c u r l} ; \Omega)$ by

$$
\begin{array}{r}
\left(\boldsymbol{\mu}^{-1} \operatorname{curl} F_{h} \mathbf{u}, \operatorname{curl} \mathbf{u}_{h}^{\prime}\right)_{\boldsymbol{L}^{2}(\Omega)}=\left(\boldsymbol{\mu}^{-1} \operatorname{curl} \mathbf{u}, \operatorname{curl} \mathbf{u}_{h}^{\prime}\right)_{\boldsymbol{L}^{2}(\Omega)} \\
\forall \mathbf{u}_{h}^{\prime} \in \boldsymbol{Z}_{h, 0}\left(\boldsymbol{\epsilon}, \Omega_{h}\right) .
\end{array}
$$

Therefore $\operatorname{curl}_{h} \mathbf{u}=\mathrm{Q}_{h}^{1 / \boldsymbol{\mu}} \operatorname{curl} \mathbf{u}$, where $\mathrm{Q}_{h}^{1 / \boldsymbol{\mu}}$ denotes the $\left(\boldsymbol{\mu}^{-1} \cdot, \cdot\right) \boldsymbol{L}^{2}(\Omega)^{-}$ orthogonal projection onto $\operatorname{curl} \mathcal{W}_{p, 0}^{1}\left(\Omega_{h}\right)$. The following theorem mirrors the approximation estimate for the Hodge mapping.

Theorem 4.8. (Fortin projector approximation estimate) If $\epsilon$ is uniformly Lipschitz-continuous, then there exist $r=r(\Omega, \boldsymbol{\mu}, \boldsymbol{\epsilon})>0$ and $C=$ $C(r, \Omega, \boldsymbol{\epsilon}, \boldsymbol{\mu})>0$ such that

$$
\left\|\mathrm{F}_{h} \mathbf{u}-\mathbf{u}\right\|_{\boldsymbol{L}^{2}(\Omega)} \leq C h^{r}\|\operatorname{curl} \mathbf{u}\|_{\boldsymbol{L}^{2}(\Omega)} \quad \forall \mathbf{u} \in \boldsymbol{Z}_{0}(\boldsymbol{\epsilon}, \Omega) .
$$

Proof. We employ a duality technique invented by D. Boffi (2000). Pick $\mathbf{u} \in$ $\boldsymbol{Z}_{0}(\boldsymbol{\epsilon}, \Omega)$ and fix $\mathbf{u}^{*} \in \boldsymbol{Z}_{0}(\boldsymbol{\epsilon}, \Omega)$ by demanding $\operatorname{curl} \mathbf{u}^{*}=\mathrm{Q}_{h}^{1 / \boldsymbol{\mu}} \operatorname{curl} \mathbf{u}$. This is meaningful because of the Poincaré-Friedrichs inequality of Corollary 4.4. First, observe that the approximation estimate of Lemma 4.5 for the Hodge mapping readily yields (for a suitable $s>0$ )

$$
\left\|\mathrm{F}_{h} \mathbf{u}-\mathbf{u}^{*}\right\|_{\boldsymbol{L}^{2}(\Omega)}=\left\|\left(\mathrm{Id}-\mathrm{H}_{\boldsymbol{\epsilon}}\right) \mathrm{F}_{h} \mathbf{u}\right\|_{\boldsymbol{L}^{2}(\Omega)} \leq C h^{s}\left\|\operatorname{curl} \mathrm{F}_{h} \mathbf{u}\right\|_{\boldsymbol{L}^{2}(\Omega)} .
$$

We proceed with a duality estimate. For $\mathbf{g} \in \boldsymbol{L}^{2}(\Omega)$ consider the saddle point problem that seeks $\mathbf{w}=\mathbf{w}(\mathbf{g}) \in \boldsymbol{H}_{0}(\operatorname{curl} ; \Omega), \mathbf{p}=\mathbf{p}(\mathbf{g}) \in \operatorname{curl} \boldsymbol{H}_{0}(\operatorname{curl} ; \Omega)$ such that

$$
\begin{aligned}
\left(\boldsymbol{\epsilon} \mathbf{w}, \mathbf{w}^{\prime}\right)_{\mathbf{L}^{2}(\Omega)}+\left(\operatorname{curl} \mathbf{w}^{\prime}, \boldsymbol{\mu}^{-1} \mathbf{p}\right)_{\mathbf{L}^{2}(\Omega)} & =0 \\
& =\left(\boldsymbol{\mu}^{-1} \mathbf{g}, \mathbf{p}^{\prime}\right)_{\boldsymbol{L}^{2}(\Omega)},
\end{aligned}
$$

for all $\mathbf{w}^{\prime} \in \boldsymbol{H}_{0}(\operatorname{curl} ; \Omega), \mathbf{p}^{\prime} \in \operatorname{curl} \boldsymbol{H}_{0}(\operatorname{curl} ; \Omega)$. As $\operatorname{curl} \boldsymbol{H}_{0}(\operatorname{curl} ; \Omega)$ is a closed subspace of $\boldsymbol{L}^{2}(\Omega)$ and the inf-sup conditions (Brezzi and Fortin 1991, Chapter 2) are trivially satisfied, we get a unique solution $(\mathbf{w}(\mathbf{g}), \mathbf{p}(\mathbf{g}))$.

Obviously, $\mathbf{w}(\mathbf{g}) \in \boldsymbol{Z}_{0}(\boldsymbol{\epsilon}, \Omega)$, and for $\mathbf{v}:=\boldsymbol{\mu}^{-1} \mathbf{p}$ we deduce from the variational equations

$$
\operatorname{curl} \mathbf{v}=-\boldsymbol{\epsilon} \mathbf{w} \in \boldsymbol{L}^{2}(\Omega), \quad \operatorname{div}(\boldsymbol{\mu} \mathbf{v})=0 \quad \text { in } \Omega, \quad \gamma_{\mathbf{n}}(\boldsymbol{\mu} \mathbf{v})=0 \quad \text { on } \partial \Omega .
$$

Moreover, since $\mathbf{p} \in \operatorname{curl} \boldsymbol{H}_{0}(\mathbf{c u r l} ; \Omega), \mathbf{v}$ is orthogonal to the space of $\boldsymbol{\mu}$ harmonic Neumann vector-fields. Since those provide all functions $\mathbf{h}$ satisfying $\operatorname{curl} \mathbf{h}=0, \operatorname{div}(\boldsymbol{\mu h})=0, \gamma_{\mathbf{n}}(\boldsymbol{\mu} \mathbf{h})=0$, we conclude from Lemma 4.2 that there is some $0<r<\frac{1}{2}, r<r_{0}, r_{0}$ from Theorem 4.2, such that

$$
\|\mathbf{v}\|_{\boldsymbol{H}^{r}(\Omega)} \leq C\left(\|\mathbf{c u r l} \mathbf{v}\|_{\boldsymbol{L}^{2}(\Omega)}+\|\operatorname{div}(\boldsymbol{\mu} \mathbf{v})\|_{L^{2}(\Omega)}\right) .
$$


The piecewise smooth matrix function $\boldsymbol{\mu}$ is a multiplier in $\boldsymbol{H}^{r}(\Omega)$. Thus, we end up with an estimate for $\mathbf{p}$ :

$$
\begin{aligned}
\|\mathbf{p}(\mathbf{g})\|_{\boldsymbol{H}^{r}(\Omega)} & \leq C\|\mathbf{v}\|_{\boldsymbol{H}^{r}(\Omega)} \leq C\left\|\operatorname{curl}\left(\boldsymbol{\mu}^{-1} \mathbf{p}\right)\right\|_{\boldsymbol{L}^{2}(\Omega)} \\
& \leq C\|\boldsymbol{\epsilon} \mathbf{w}\|_{\boldsymbol{L}^{2}(\Omega)} \leq C\|\operatorname{curl} \mathbf{w}\|_{\boldsymbol{L}^{2}(\Omega)} \leq C\|\mathbf{g}\|_{\boldsymbol{L}^{2}(\Omega)},
\end{aligned}
$$

where we used Corollary 4.4 in the penultimate step. From here on, the generic constants are allowed to depend on the material parameters $\boldsymbol{\epsilon}$ and $\boldsymbol{\mu}$.

In the spirit of classical duality techniques, we find $\mathbf{u}-\mathbf{u}^{*}=\mathbf{w}(\mathbf{g})$, if $\mathbf{g}=\left(\mathrm{Id}-\mathrm{Q}_{h}^{1 / \boldsymbol{\mu}}\right) \mathbf{c u r l} \mathbf{u}$. From the saddle point problem we can extract

$$
\begin{aligned}
\left\|\mathbf{u}-\mathbf{u}^{*}\right\|_{\boldsymbol{L}^{2}(\Omega)} & \leq C\left(\boldsymbol{\epsilon}\left(\mathbf{u}-\mathbf{u}^{*}\right), \mathbf{u}-\mathbf{u}^{*}\right)_{\mathbf{L}^{2}(\Omega)} \\
& \leq C\left(\boldsymbol{\mu}^{-1} \mathbf{p}, \operatorname{curl}\left(\mathbf{u}-\mathbf{u}^{*}\right)\right)_{\boldsymbol{L}^{2}(\Omega)} \\
& \leq C\left(\boldsymbol{\mu}^{-1}\left(\operatorname{Id}-\Pi_{p}^{2}\right) \mathbf{p},\left(\operatorname{Id}-\mathrm{Q}_{h}^{1 / \boldsymbol{\mu}}\right) \operatorname{curl} \mathbf{u}\right)_{\boldsymbol{L}^{2}(\Omega)} .
\end{aligned}
$$

This is legal, because by (3.39) the interpolant $\Pi_{p}^{2} \mathbf{p}$ is well defined. As, by Lemma 2.4, there exists $\mathbf{q} \in \boldsymbol{H}^{1}(\Omega) \cap \boldsymbol{H}_{0}(\mathbf{c u r l} ; \Omega)$ such that $\mathbf{p}=\mathbf{c u r l} \mathbf{q}$, the commuting diagram property gives

$$
\Pi_{p}^{2} \mathbf{p}=\operatorname{curl} \Pi_{p}^{1} \mathbf{q} \in \operatorname{curl} \mathcal{W}_{p, 0}^{1}\left(\Omega_{h}\right) .
$$

Finally, the properties of $\mathrm{Q}_{h}^{1 / \boldsymbol{\mu}}$ justify the above manipulations. The proof is finished by using Theorem 3.16,

$$
\begin{aligned}
\left\|\mathbf{u}-\mathbf{u}^{*}\right\|_{\boldsymbol{L}^{2}(\Omega)} & \leq C\left\|\left(\operatorname{Id}-\Pi_{p}^{2}\right) \mathbf{p}\right\|_{\boldsymbol{L}^{2}(\Omega)}\left\|\boldsymbol{\mu}^{-\frac{1}{2}}\left(\operatorname{Id}-\mathrm{Q}_{h}^{1 / \boldsymbol{\mu}}\right) \operatorname{curl} \mathbf{u}\right\|_{\boldsymbol{L}^{2}(\Omega)} \\
& \leq C h^{r}\|\mathbf{p}\|_{\boldsymbol{H}^{r}(\Omega)}\left\|\boldsymbol{\mu}^{-\frac{1}{2}} \operatorname{curl} \mathbf{u}\right\|_{\boldsymbol{L}^{2}(\Omega)} \\
& \leq C h^{r}\|\operatorname{curl} \mathbf{u}\|_{\boldsymbol{L}^{2}(\Omega)}
\end{aligned}
$$

and taking into account (4.11).

Compared to the local interpolation operator $\Pi_{p}^{1}$, the big advantage of the Fortin projector $F_{h}$ is that it is well defined on all of $\boldsymbol{H}_{0}(\operatorname{curl} ; \Omega)$. For both projectors $\operatorname{Ker}$ (curl) (restricted to their domains) is an invariant subspace. Yet properties of $F_{h}$ are much more difficult to establish because of its nonlocal nature.

Remark 13. The assumption that $\epsilon$ be uniformly Lipschitz-continuous is very restrictive, because the common case of $\Omega$ being filled with different dielectric materials invariably leads to discontinuous $\boldsymbol{\epsilon}$. At present we need this assumption, in order to be able to apply Lemma 4.6. It seems likely that the statement of this lemma can be extended to less regular vectorfields by following the lines of the proofs of Theorem 4.1 and of Theorem 3.5 
in Costabel et al. (1999), and using information about interface singularities of solutions of the Dirichlet boundary value problem for $\operatorname{div}(\epsilon \operatorname{grad} \cdot)$.

\subsection{Error estimates}

First, owing to the discrete Poincaré-Friedrichs inequality of Theorem 4.7, we can define a meaningful operator $\mathrm{T}_{h}: \boldsymbol{L}^{2}(\Omega) \mapsto \boldsymbol{Z}_{h, 0}\left(\boldsymbol{\epsilon}, \Omega_{h}\right)$ by

$$
\left(\boldsymbol{\mu}^{-1} \operatorname{curl} \mathbf{T}_{h} \mathbf{u}, \operatorname{curl} \mathbf{u}_{h}^{\prime}\right)_{\boldsymbol{L}^{2}(\Omega)}=\left(\boldsymbol{\epsilon} \mathbf{u}, \mathbf{u}_{h}^{\prime}\right)_{\boldsymbol{L}^{2}(\Omega)} \quad \forall \mathbf{u}_{h}^{\prime} \in \mathcal{W}_{p, 0}^{1}\left(\Omega_{h}\right)
$$

In particular, this implies

$$
\left(\boldsymbol{\mu}^{-1} \operatorname{curl}\left(\mathrm{T}_{h}-\mathrm{T}\right) \mathbf{u}, \operatorname{curl} \mathbf{u}_{h}^{\prime}\right)_{\boldsymbol{L}^{2}(\Omega)}=0 \quad \forall \mathbf{u}_{h}^{\prime} \in \mathcal{W}_{p, 0}^{1}\left(\Omega_{h}\right),
$$

which can be expressed through the Fortin projector as

$$
\mathrm{T}_{h}=\mathrm{F}_{h} \circ \mathrm{T} \text {. }
$$

Thus, Theorem 4.8 and the continuity of $\mathrm{T}$ lead to

$$
\begin{aligned}
\left\|\left(\mathrm{T}-\mathrm{T}_{h}\right) \mathbf{u}\right\|_{\boldsymbol{L}^{2}(\Omega)} & \leq\left\|\left(\mathrm{Id}-\mathrm{F}_{h}\right) \mathrm{T} \mathbf{u}\right\|_{\boldsymbol{L}^{2}(\Omega)} \\
& \leq C h^{r}\|\mathbf{c u r l} \mathrm{Tu}\|_{\boldsymbol{L}^{2}(\Omega)} \leq C h^{r}\|\mathbf{u}\|_{\boldsymbol{L}^{2}(\Omega)}
\end{aligned}
$$

for all $\mathbf{u} \in \boldsymbol{L}^{2}(\Omega)$ and some $r>0$, provided that $\boldsymbol{\epsilon}$ is uniformly Lipschitzcontinuous. Under this assumption we conclude uniform convergence $\mathrm{T}_{h} \stackrel{h \rightarrow 0}{\longrightarrow}$ $\mathrm{T}$ in $\boldsymbol{L}^{2}(\Omega)$, that is,

$$
\left\|\mathrm{T}-\mathrm{T}_{h}\right\|_{\boldsymbol{L}^{2}(\Omega) \rightarrow \boldsymbol{L}^{2}(\Omega)} \leq C h^{r} \rightarrow 0 \quad \text { for } h \rightarrow 0 .
$$

This paves the way for applying the powerful results on spectral approximation put forth in Babuška and Osborn (1991, Section 7), because a key assumption of this theory is that of uniform convergence $\mathrm{T}_{h} \stackrel{h \rightarrow 0}{\longrightarrow} \mathrm{T}$. Writing $\mathbf{v}_{1}, \ldots, \mathbf{v}_{m}$ for the orthonormalized eigenfunctions of $\mathrm{T}$ belonging to an eigenvalue $\omega>0$ of multiplicity $m \in \mathbb{N}$, we conclude from Babuška and Osborn (1991, Theorem 7.3) that on sufficiently fine meshes we will find $m$ discrete eigenvalues $\omega_{1}, \ldots, \omega_{m}$ such that

$$
\begin{aligned}
\left|\omega^{-2}-\omega_{k}^{-2}\right| \leq C\left(\sum_{n, l=1}^{m}\right. & \left(\left(\mathrm{T}-\mathrm{T}_{h}\right) \mathbf{v}_{n}, \mathbf{v}_{l}\right)_{\boldsymbol{L}^{2}(\Omega)} \\
& \left.+\left\|\left(\mathrm{T}-\mathrm{T}_{h}\right)_{\mid \operatorname{Span}\left\{\mathbf{v}_{1}, \ldots, \mathbf{v}_{m}\right\}}\right\|_{\boldsymbol{L}^{2}(\Omega) \rightarrow \boldsymbol{L}^{2}(\Omega)}^{2}\right) .
\end{aligned}
$$

Moreover, for each $\mathbf{v}_{k}$ we find a discrete eigenfunction $\mathbf{v}_{h}$ such that

$$
\left\|\mathbf{v}_{k}-\mathbf{v}_{h}\right\|_{\boldsymbol{L}^{2}(\Omega)} \leq C\left\|\left(\mathrm{~T}-\mathrm{T}_{h}\right)_{\mid \operatorname{Span}\left\{\mathbf{v}_{1}, \ldots, \mathbf{v}_{m}\right\}}\right\|_{\boldsymbol{L}^{2}(\Omega) \rightarrow \boldsymbol{L}^{2}(\Omega)} .
$$

In both cases the constants are independent of the choice of the finite element spaces. 
Hence, information about the convergence of eigenvalues can be obtained from studying the pointwise convergence $\mathrm{T}_{h} \rightarrow \mathrm{T}$. The latter can be derived from the following saddle point problem. Seek $\mathrm{Tu} \in \boldsymbol{H}_{0}(\operatorname{curl} ; \Omega), \mathbf{p} \in$ $\boldsymbol{H}_{0}(\operatorname{curl} 0 ; \Omega)$ such that

$$
\begin{aligned}
\left(\boldsymbol{\mu}^{-1} \operatorname{curl} \mathbf{T} \mathbf{u}, \operatorname{curl} \mathbf{v}^{\prime}\right)_{\boldsymbol{L}^{2}(\Omega)}+\left(\mathbf{v}^{\prime}, \mathbf{p}\right)_{\mathbf{L}^{2}(\Omega)} & =\left(\boldsymbol{\epsilon} \mathbf{u}, \mathbf{v}^{\prime}\right)_{\mathbf{L}^{2}(\Omega)}, \\
\left(\mathrm{T} \mathbf{u}, \mathbf{p}^{\prime}\right)_{\boldsymbol{L}^{2}(\Omega)} & =0,
\end{aligned}
$$

for all $\mathbf{v}^{\prime} \in \boldsymbol{H}_{0}(\operatorname{curl} ; \Omega), \mathbf{p}^{\prime} \in \boldsymbol{H}_{0}(\operatorname{curl} 0 ; \Omega)$. The discrete PoincaréFriedrichs inequality guarantees that the Galerkin discretization of (4.15) based on $\mathcal{W}_{p}^{1}\left(\Omega_{h}\right)$ and $\mathcal{W}_{p}^{1}\left(\Omega_{h}\right) \cap \operatorname{Ker}(\mathbf{c u r l})$ will satisfy 'ellipticity on the kernel', the critical one of the LBB-conditions for (4.15) (Brezzi and Fortin 1991, Chapter 2). Please be aware that in (4.13) and (4.14) only eigenvectors occur as arguments to $\mathrm{T}$ and $\mathrm{T}_{h}$. These belong to $\boldsymbol{Z}_{0}(\boldsymbol{\epsilon}, \Omega)$ and this makes the solution for $\mathbf{p}$ and its discrete approximations vanish. Summing up, for $\mathbf{v} \in \boldsymbol{Z}_{0}(\boldsymbol{\epsilon}, \Omega)$ we get the asymptotic a priori error estimate

$$
\left\|\left(\mathrm{T}-\mathrm{T}_{h}\right) \mathbf{v}\right\|_{\boldsymbol{H}(\mathbf{c u r l} ; \Omega)} \leq C \inf _{\mathbf{u}_{h} \in \mathcal{W}_{p}^{1}\left(\Omega_{h}\right)}\left\|\mathrm{T} \mathbf{v}-\mathbf{u}_{h}\right\|_{\boldsymbol{H}(\operatorname{curl} ; \Omega)},
$$

where $C>0$ depends on the constant in Corollary 4.7 and the material parameters. Ultimately the rate of convergence of eigenvalues and eigenvectors will be governed by the smoothness of the eigenvectors. Its discussion will be postponed until the end of Section 5.2. One might object that using (4.16) gives sub-optimal estimates, since the $\boldsymbol{L}^{2}(\Omega)$-norm should be targeted. However, it seems that better estimates for the $\boldsymbol{L}^{2}(\Omega)$-norm are hard to get.

\section{Bibliographical notes}

The above plan for analysing the Maxwell eigenvalue problem was first applied in Boffi, Fernandes, Gastaldi and Perugia (1999), based on techniques of Boffi, Brezzi and Gastaldi (2000). The Fortin projector $F_{h}$ was introduced in Boffi (2000).

\subsection{Discrete compactness}

The use of the Fortin projector to tackle the Maxwell eigenvalue problem is fairly recent. The first successful convergence analysis of Kikuchi (1989) pursued a different course and employed the notions of discrete compactness and collective compactness (Anselone 1971). They permit us to reduce convergence of eigenvalues/eigenfunctions to the pointwise convergence $\mathrm{T}_{h} \rightarrow \mathrm{T}$ without a detour via uniform convergence. It will turn out that thus we can get rid of the restrictive assumptions on $\epsilon$ that were necessary in the previous section. It should be stressed that discrete compactness is a very interesting 
property of discrete 1-forms in its own right, important beyond the analysis of eigenvalue problems.

However, the arguments are based on sequential compactness. Therefore, they always target a family $\left(\Omega_{h}\right)_{h \in \mathbb{H}}$ of triangulations, for which the sequence $\mathbb{H}$ of meshwidths is decreasing and converging to 0 . Moreover, $\rho\left(\Omega_{h}\right), h \in$ $\mathbb{H}$, is to be bounded uniformly in $h$. In the terminology of Ciarlet (1978, Section 3.2) this characterizes a shape-regular family of meshes.

Theorem 4.9. (Discrete compactness property) Let $\left(\Omega_{h}\right)_{h \in \mathbb{H}}$ be a uniformly shape-regular family of triangulations of $\Omega$ with meshwidths tending to 0 . Any sequence $\left(\mathbf{u}_{h}\right)_{h \in \mathbb{H}}$ with $\mathbf{u}_{h} \in \boldsymbol{Z}_{h, 0}\left(\boldsymbol{\epsilon}, \Omega_{h}\right)$ that is uniformly bounded in $\boldsymbol{H}(\mathbf{c u r l} ; \Omega)$ contains a subsequence that converges strongly in $\boldsymbol{L}^{2}(\Omega)$.

Proof. (See the proof of Theorem 4.1 in Kirsch and Monk (2000), and Caorsi, Fernandes and Raffetto (2000).) As in the proof of Theorem 4.7 we first look at the $\boldsymbol{L}^{2}(\Omega)$-orthogonal Helmholtz decompositions

$$
\mathbf{u}_{h}= \begin{cases}\mathbf{z}^{h}+\mathbf{q}^{h}, & \mathbf{z}^{h} \in \boldsymbol{Z}_{0}(\mathbf{I}, \Omega), \operatorname{curl} \mathbf{q}^{h}=0, \\ \mathbf{z}_{h}^{h}+\mathbf{q}_{h}^{h}, & \mathbf{z}_{h}^{h} \in \boldsymbol{Z}_{h, 0}\left(\mathbf{I}, \Omega_{h}\right), \operatorname{curl} \mathbf{q}_{h}^{h}=0 .\end{cases}
$$

As both Helmholtz decompositions are uniformly stable in the $\boldsymbol{H}(\mathbf{c u r l} ; \Omega)$ norm, $\left(\mathbf{z}^{h}\right)_{h \in \mathbb{H}}$ is a bounded sequence in $\boldsymbol{Z}_{0}(\mathbf{I}, \Omega)$. Hence, by Corollary 4.3, it possesses a subsequence, still denoted by $\left(\mathbf{z}^{h}\right)_{h \in \mathbb{H}}$, that converges in $\boldsymbol{L}^{2}(\Omega)$ to some $\mathbf{z} \in \boldsymbol{L}^{2}(\Omega)$ as $h \rightarrow 0$. Appealing to Lemma 4.5 we conclude that $\left\|\mathbf{z}_{h}-\mathbf{z}_{h}^{h}\right\|_{\boldsymbol{L}^{2}(\Omega)} \rightarrow 0$. Hence, $\left(\mathbf{z}_{h}^{h}\right)_{h \in \mathbb{H}}$ must converge in $\boldsymbol{L}^{2}(\Omega)$ to the same $\operatorname{limit} \mathbf{z}$ as $\left(\mathbf{z}^{h}\right)_{h \in \mathbb{H}}$.

By definition of $\boldsymbol{Z}_{h, 0}\left(\boldsymbol{\epsilon}, \Omega_{h}\right)$,

$$
\left(\boldsymbol{\epsilon} \mathbf{q}_{h}^{h}, \mathbf{p}_{h}\right)_{\boldsymbol{L}^{2}(\Omega)}=-\left(\boldsymbol{\epsilon} \mathbf{z}_{h}^{h}, \mathbf{p}_{h}\right)_{\boldsymbol{L}^{2}(\Omega)} \quad \forall \mathbf{p}_{h} \in \operatorname{Ker}(\mathbf{c u r l}) \cap \mathcal{W}_{p, 0}^{1}\left(\Omega_{h}\right) .
$$

This can be regarded as a perturbed Galerkin approximation of the following continuous variational problem. Seek $\mathbf{q} \in \boldsymbol{H}_{0}(\operatorname{curl} 0 ; \Omega)$ such that

$$
(\boldsymbol{\epsilon} \mathbf{q}, \mathbf{p})_{\boldsymbol{L}^{2}(\Omega)}=-(\boldsymbol{\epsilon \mathbf { Z }}, \mathbf{p})_{\boldsymbol{L}^{2}(\Omega)} \quad \forall \mathbf{p} \in \boldsymbol{H}_{0}(\operatorname{curl} 0 ; \Omega) .
$$

Strang's lemma (Ciarlet 1978, Theorem 4.4.1) teaches us that

$$
\left\|\mathbf{q}-\mathbf{q}_{h}^{h}\right\|_{\boldsymbol{L}^{2}(\Omega)} \leq C\left(\inf _{\mathbf{v}_{h} \in \mathcal{W}_{p, 0}^{1}\left(\Omega_{h}\right) \cap \operatorname{Ker}(\mathbf{c u r l})}\left\|\mathbf{q}-\mathbf{v}_{h}\right\|_{L^{2}(\Omega)}+\left\|\mathbf{z}_{h}^{h}-\mathbf{z}\right\|_{\boldsymbol{L}^{2}(\Omega)}\right),
$$

where $C>0$ depends only on $\boldsymbol{\epsilon}$. Next, recall that there is a representation

$$
\mathbf{q}=\operatorname{grad} \phi+\mathbf{h}, \quad \phi \in H_{0}^{1}(\Omega), \mathbf{h} \in \mathcal{H}^{2}(\Omega) .
$$

The cohomology space, by orthogonalization with respect to all gradients, 
can always be chosen to be a subspace of $\boldsymbol{X}_{0}(\mathbf{I}, \Omega) \cap \boldsymbol{H}_{0}(\operatorname{curl} 0 ; \Omega)$. Therefore, $\Pi_{p}^{1} \mathbf{h}$ makes sense. From Theorem 4.1 in combination with Lemma 4.6 we learn that $\Pi_{p}^{1} \mathbf{h}$ converges to $\mathbf{h}$ in $\boldsymbol{L}^{2}(\Omega)$.

It is also known that $\bigcup_{h \in \mathbb{H}} \mathcal{W}_{p, 0}^{0}\left(\Omega_{h}\right)$ is dense in $H_{0}^{1}(\Omega)$ (Schatz and Wang 1996). Hence, $\mathbf{v}_{h}$ in (4.17) can be built from the gradient of the best approximation of $\phi$ in $\mathcal{W}_{p, 0}^{0}\left(\Omega_{h}\right)$ and the interpolant $\Pi_{p}^{1} \mathbf{h}$. This will achieve $\left\|\mathbf{q}-\mathbf{v}_{h}\right\|_{\boldsymbol{L}^{2}(\Omega)} \rightarrow 0$ as $h \rightarrow 0$. As a consequence, we also have $\mathbf{q}_{h}^{h} \rightarrow \mathbf{q}$ in $\boldsymbol{L}^{2}(\Omega)$. Summing up, $\mathbf{u}_{h} \rightarrow \mathbf{z}+\mathbf{q}$ in $\boldsymbol{L}^{2}(\Omega)$ for $h \rightarrow 0$.

Remark 14. It is hardly surprising that the discrete Friedrich's inequality is implied by the discrete compactness property (Monk and Demkowicz 2001, Caorsi et al. 2000).

The discrete compactness property enables us to use the theory of collectively compact operators (Kress 1989, Section 10.3). We recall the principal definition.

Definition 6. A family $\mathcal{A}$ of linear operators mapping a normed space $\mathcal{X}$ into a normed space $\mathcal{Y}$ is called collectively compact, if, for each bounded set $U \subset \mathcal{X}$, the image $\mathcal{A}(U):=\{A x, x \in U, A \in \mathcal{A}\}$ is relatively compact in $\mathcal{Y}$.

Theorem 4.10. (Collective compactness of $T_{h}$ ) On a shape-regular family of meshes with meshwidth $h \in \mathbb{H}$ tending to zero, the family $\left\{T_{h}\right\}_{h \in \mathbb{H}}$ of operators is collectively compact.

Proof. The proof is adapted from Monk and Demkowicz (2001). We first take a look at a sequence $\left(\mathbf{w}_{n}\right)_{n \in \mathbb{N}}$ in the space

$$
\mathcal{W}:=\bigcup_{h} Z_{h, 0}\left(\epsilon, \Omega_{h}\right) \subset \boldsymbol{H}(\operatorname{curl} ; \Omega)
$$

that is bounded in $\boldsymbol{H}_{0}(\mathbf{c u r l} ; \Omega)$. Write $h_{n}$ for the largest meshwidth $\in \mathbb{H}$ for which $\mathbf{w}_{n}$ belongs to $\boldsymbol{Z}_{h, 0}\left(\boldsymbol{\epsilon}, \Omega_{h}\right)$. If the set $\left\{h_{n}\right\}_{n \in \mathbb{N}}$ is finite, the sequence $\left(\mathbf{w}_{n}\right)_{n}$ is contained in a space of finite dimension and must have a convergent subsequence in $\boldsymbol{L}^{2}(\Omega)$.

Otherwise, we can assume $h_{n} \rightarrow 0$ as $n \rightarrow \infty$. By the discrete compactness property we may extract a subsequence that converges in $\boldsymbol{L}^{2}(\Omega)$. Thus, it is immediate that $\mathcal{W}$ is compactly embedded in $\boldsymbol{L}^{2}(\Omega)$.

Next, let $U$ be a bounded set in $\boldsymbol{L}^{2}(\Omega)$. As

$$
\left\|\operatorname{curl} \mathrm{T}_{h} \mathbf{f}\right\|_{\boldsymbol{L}^{2}(\Omega)} \leq C\|\mathbf{f}\|_{\boldsymbol{L}^{2}(\Omega)}
$$

with $C>0$ depending only on $\boldsymbol{\epsilon}$ and $\boldsymbol{\mu}$, the set

$$
\left\{\mathrm{T}_{h} \mathbf{f}, h \in \mathbb{H}, \mathbf{f} \in U\right\}
$$

is bounded in $\mathcal{W}$. Therefore, it must be relatively compact in $\boldsymbol{L}^{2}(\Omega)$. 
Citing Theorems 4 and 5 from Osborn (1975), collective compactness of $\left(\mathrm{T}_{h}\right)_{h \in \mathbb{H}}$ along with the pointwise convergence $\mathrm{T}_{h} \stackrel{h \rightarrow 0}{\longrightarrow} \mathrm{T}$, established at the end of the previous section, gives us that the estimates (4.13) and (4.14) still hold for an arbitrary metric tensor $\boldsymbol{\epsilon}$.

Remark 15. A priori convergence estimates for the $p$-version of discrete 1-forms applied to the variational eigenvalue problem (4.1) could not be obtained up to now. Apart from gaps in the theory of $p$-version interpolation error estimates (see Section 3.6), the main obstacle is that Lemma 4.6 cannot be directly adapted to a $p$-version setting. In particular, writing $T$ for a fixed tetrahedron, one would need there to exist $r>0$ and a positive constant $C=C(T)$, such that

$$
\left\|\mathbf{u}-\Pi_{p}^{1} \mathbf{u}\right\|_{\boldsymbol{L}^{2}(T)} \leq C p^{-r}\|\mathbf{u}\|_{\boldsymbol{H}^{s}(T)}
$$

for all $\mathbf{u} \in \boldsymbol{H}^{s}(T) \cap \boldsymbol{H}(\mathbf{c u r l} ; T), s>\frac{1}{2}$, for which curl u is contained in $\mathcal{W}_{p}^{2}(T)$.

Bibliographical notes

A profound analysis of the Maxwell eigenvalue problem, giving sufficient and necessary conditions for viable $\boldsymbol{H}(\mathbf{c u r l} ; \Omega)$-conforming finite element spaces, is presented in Caorsi, Fernandes and Raffetto (1999, 2000). A less technical discussion is given in Fernandes and Raffetto (2000). Discrete compactness for edge elements is also a core subject in Monk and Demkowicz (2001) and Demkowicz et al. (1999).

\subsection{Lagrangian finite elements}

There is a big temptation raised by looking at the variational formulation (4.1) only, oblivious of the subtle algebraic relationships between the electromagnetic fields. Then one might argue that any $\boldsymbol{H}(\mathbf{c u r l} ; \Omega)$-conforming finite element space on $\Omega_{h}$ can be used for the sake of Galerkin approximation, provided that any $\mathbf{u} \in \boldsymbol{H}_{0}(\mathbf{c u r l} ; \Omega)$ can be approximated arbitrarily well for $h \rightarrow 0$. In particular, piecewise linear, globally continuous finite elements might be used as trial spaces for the Cartesian components of $\mathbf{u}$. However, this is strongly deprecated for two reasons.

(1) Thanks to the discrete Poincaré-Friedrichs inequality, the nonzero discrete eigenvalues are bounded away from zero, if discrete differential forms are used. This cannot be guaranteed for componentwise discretization with Lagrangian finite elements. In general, the kernel of curl will give rise to numerous nonzero discrete eigenvalues swamping the discrete spectrum. 

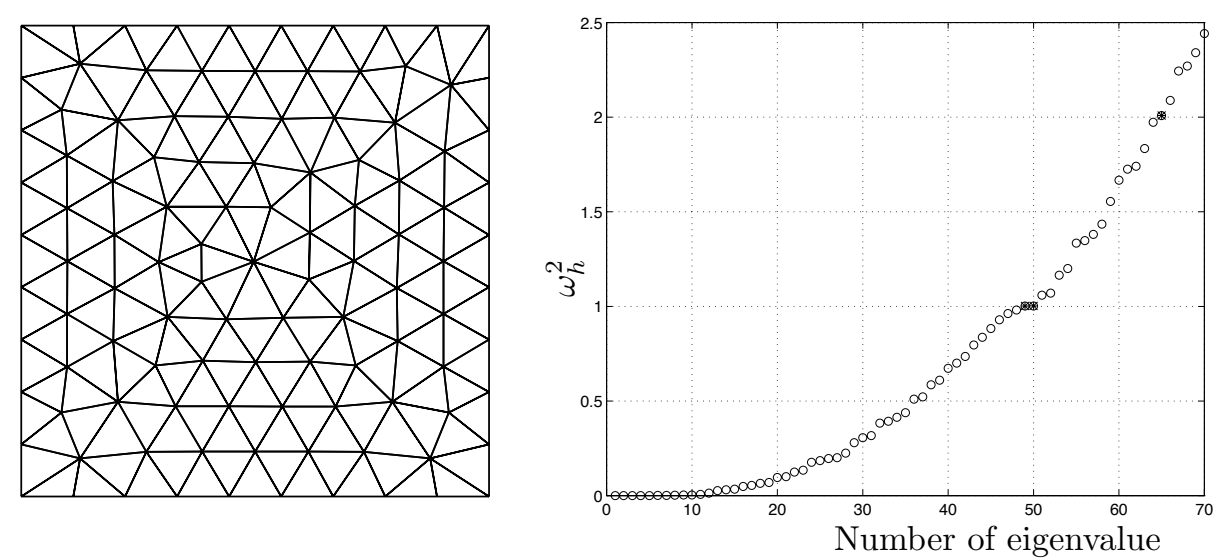

Figure 4.2. Numerical experiment for the 2D Maxwell eigenvalue problem on the unit square. Componentwise discretization of the field by means of Lagrangian finite elements on an unstructured mesh (left) was employed. (Figure 8 in Boffi et al. (1999), courtesy of D. Boffi, F. Fernandes, L. Gastaldi, and I. Perugia)
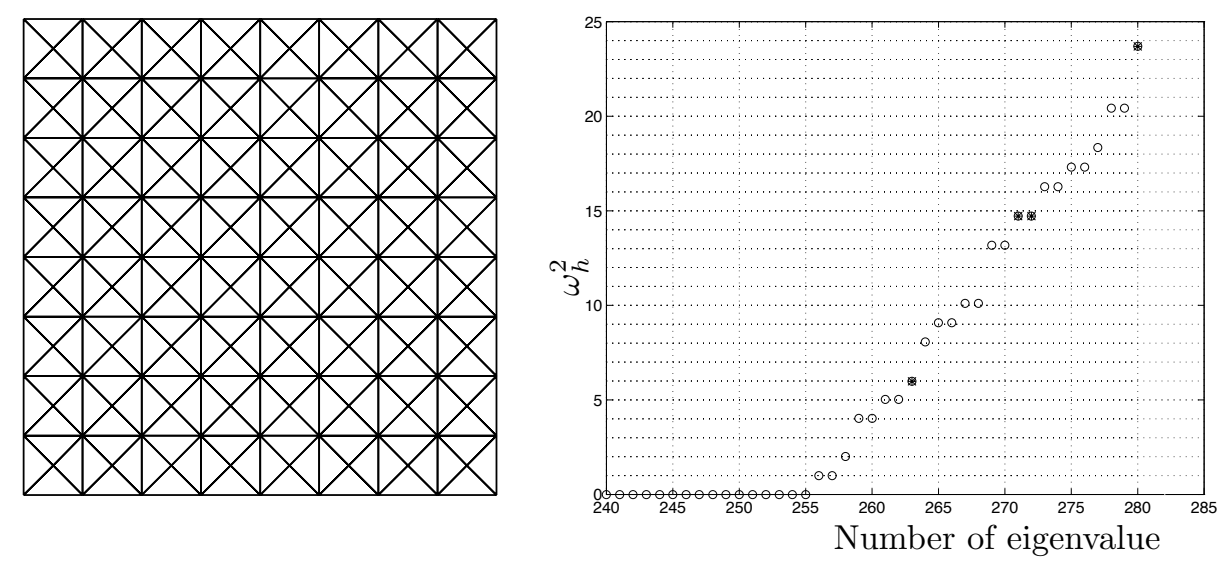

Figure 4.3. Discrete eigenvalues of the 2D Maxwell eigenvalue problem on the unit square discretized by means of Lagrangian finite elements on a 'criss-cross' mesh (left) for the components of e. (Figure 10 in Boffi et al. (1999), courtesy of D. Boffi, F. Fernandes, L. Gastaldi, and I. Perugia)

(2) On special meshes it might be possible to capture the kernel of curl by continuous finite elements. This will keep the smallest nonzero eigenvalue uniformly bounded away from zero. Yet in this case perilous spurious eigenvalues might still arise. They are dotted among correct eigenvalues and it seems impossible to single them out. 
Numerical computations confirming these statements are reported in Boffi et al. (1999) and here we restate the striking findings. For the sake of simplicity a two-dimensional situation was considered by assuming translation invariance with respect to one spatial direction. This means that $\mathbf{e}=\left(E_{1}, E_{2}\right)$ and curle $=\partial_{1} E_{2}-\partial_{2} E_{1}$ in (4.1). The discrete spectrum for a truly unstructured mesh is given in Figure 4.2. A glaring pollution effect occurs: most of the small eigenvalues (circles in Figure 4.2) should actually be zero. The computed eigenvalues for a special 'criss-cross' grid that allows the resolution of the discrete kernel are plotted in Figure 4.3. Eigenvalues that have no continuous counterpart ('spurious eigenvalues') are marked by solid squares, whereas circles represent correct approximations of eigenvalues.

\section{Maxwell source problem}

As in the case of the Maxwell eigenvalue problem, we consider a bounded cavity $\Omega \subset A\left(\mathbb{R}^{3}\right)$ with piecewise smooth Lipschitz boundary, on which PEC boundary conditions are imposed. The cavity is filled with a non-conducting dielectric medium described by piecewise smooth material parameters $\boldsymbol{\epsilon}$ and $\boldsymbol{\mu}$. We rely on the framework developed in Section 2.3 and assume that electromagnetic fields inside $\Omega$ are excited by a time-harmonic source current $\mathbf{j}$ with a fixed angular frequency $\omega>0$.

We focus on the e-based primal variational formulation (2.28) of the following boundary value problem. For $\mathbf{j} \in \boldsymbol{L}^{2}(\Omega)$ seek $\mathbf{e} \in \boldsymbol{H}_{0}(\mathbf{c u r l} ; \Omega)$ such that, for all $\mathbf{e}^{\prime} \in \boldsymbol{H}_{0}(\operatorname{curl} ; \Omega)$,

$$
\left(\boldsymbol{\mu}^{-1} \operatorname{curl} \mathbf{e}, \operatorname{curl} \mathbf{e}^{\prime}\right)_{\boldsymbol{L}^{2}(\Omega)}-\omega^{2}\left(\boldsymbol{\epsilon} \mathbf{e}, \mathbf{e}^{\prime}\right)_{\boldsymbol{L}^{2}(\Omega)}=-i \omega\left(\mathbf{j}, \mathbf{e}^{\prime}\right)_{\boldsymbol{L}^{2}(\Omega)} .
$$

Of course, an equivalent dual $\mathbf{h}$-based formulation is also possible and it arises from (2.29). Then the PEC case amounts to imposing natural boundary conditions and the variational problem has to be posed on the space $\boldsymbol{H}(\mathbf{c u r l} ; \Omega)$. In order to get a well-posed problem, we make the following assumption.

Assumption 1. The angular frequency $\omega$ is distinct from any resonant frequency (Maxwell eigenvalue) of the dielectric cavity $\Omega$.

This assumption is equivalent to demanding that the variational problem (5.1) has at most one solution. The Galerkin discretization of (5.1) by means of discrete 1-forms is straightforward. Seek $\mathbf{e}_{h} \in \mathcal{W}_{p, 0}^{1}\left(\Omega_{h}\right)$ such that, for all $\mathbf{e}_{h}^{\prime} \in \mathcal{W}_{p, 0}^{1}\left(\Omega_{h}\right)$

$$
\left(\boldsymbol{\mu}^{-1} \operatorname{curl} \mathbf{e}_{h}, \operatorname{curl} \mathbf{e}_{h}^{\prime}\right)_{\boldsymbol{L}^{2}(\Omega)}-\omega^{2}\left(\boldsymbol{\epsilon} \mathbf{e}_{h}, \mathbf{e}_{h}^{\prime}\right)_{\boldsymbol{L}^{2}(\Omega)}=-i \omega\left(\mathbf{j}, \mathbf{e}_{h}^{\prime}\right)_{\boldsymbol{L}^{2}(\Omega)} .
$$

As already indicated by the notation, the focus will be on the $h$-version of discrete 1-forms with uniform polynomial degree $p \in \mathbb{N}_{0}$. For the sake of 
brevity, the sesqui-linear form on the left-hand side of $(5.1) /(5.2)$ will be denoted by $a(\cdot, \cdot)$.

We aim at showing asymptotically optimal convergence of the energy norm of the discretization error $\mathbf{e}-\mathbf{e}_{h}$ in the sense that, on sufficiently fine meshes,

$$
\left\|\mathbf{e}-\mathbf{e}_{h}\right\|_{\boldsymbol{H}(\operatorname{curl} ; \Omega)} \leq C \inf _{\mathbf{v}_{h} \in \mathcal{W}_{p}^{1}\left(\Omega_{h}\right)}\left\|\mathbf{e}-\mathbf{v}_{h}\right\|_{\boldsymbol{H}(\operatorname{curl} ; \Omega)}
$$

with $C>0$ only depending on parameters of the continuous problem and the shape regularity measure of $\Omega_{h}$. Note that $a(\cdot, \cdot)$ is Hermitian but obviously indefinite, which thwarts very simple convergence estimates based on $\boldsymbol{H}(\mathbf{c u r l} ; \Omega)$-ellipticity and Cea's lemma.

\subsection{Coercivity}

Let us take a brief look at the closely related Dirichlet problem for the Helmholtz equation. For $f \in L^{2}(\Omega)$ seek $\varphi \in H_{0}^{1}(\Omega)$ such that, for all $\varphi^{\prime} \in H_{0}^{1}(\Omega)$,

$$
\left(\operatorname{grad} \varphi, \operatorname{grad} \varphi^{\prime}\right)_{\boldsymbol{L}^{2}(\Omega)}-\omega^{2}\left(\varphi, \varphi^{\prime}\right)_{\boldsymbol{L}^{2}(\Omega)}=\left(f, \varphi^{\prime}\right)_{\boldsymbol{L}^{2}(\Omega)}
$$

A priori error estimates for finite element schemes for this problem usually employ (i) the coercivity of the underlying sesqui-linear form $a_{H}(\cdot, \cdot)$, that is, the fact that the zero-order term is a compact perturbation of the secondorder term, the principal part, and (ii) a Gårding inequality of the form

$$
\left|a_{H}(\varphi, \varphi)\right| \geq C\|\varphi\|_{H^{1}(\Omega)}^{2}-C^{\prime}\|\varphi\|_{L^{2}(\Omega)}^{2} \quad \forall \varphi \in H_{0}^{1}(\Omega) .
$$

This is definitely not the case for $a(\cdot, \cdot)$ from $(5.1)$, which has to be blamed on the infinite-dimensional kernel of the curl-operator. We can view the situation from a different angle. In electromagnetism the electric and magnetic energies of a field are perfectly symmetric. None can be relegated to a compact perturbation of the other. On the contrary, in acoustics, which is described by the Helmholtz equation, the two energies involved are potential energy and kinetic energy and one of them can be identified as the principal energy.

In the case of the eigenvalue problem of Section 4 , we found a remedy by restricting the variational problem to $\boldsymbol{Z}_{0}(\boldsymbol{\epsilon}, \Omega)$. Thus $\left(\boldsymbol{\mu}^{-1} \operatorname{curl} \cdot, \operatorname{curl} \cdot\right)_{\boldsymbol{L}^{2}(\Omega)}$ could be promoted to a principal part, whereas $(\boldsymbol{\epsilon} \cdot, \cdot)_{\boldsymbol{L}^{2}(\Omega)}$ played the role of a compact perturbation. For the source problem the solution e does not necessarily belong to $\boldsymbol{Z}_{0}(\boldsymbol{\epsilon}, \Omega)$. Yet the idea is still fruitful, when refined into the splitting principle:

Consider the Maxwell source problem on fields decomposed into components on which $\left(\boldsymbol{\mu}^{-1} \mathbf{c u r l} \cdot, \mathbf{c u r l} \cdot\right)_{\boldsymbol{L}^{2}(\Omega)}$ becomes a principal part, and other components, usually $\operatorname{Ker}(\mathbf{c u r l})$, on which the zero-order terms in $a(\cdot, \cdot)$ are 'principal'. 
It is natural to retain a close relative of $\boldsymbol{Z}_{0}(\boldsymbol{\epsilon}, \Omega)$ as the first component in such a decomposition. Therefore, we consider the classical $\boldsymbol{L}^{2}(\Omega)$-orthogonal Helmholtz decomposition

$$
\boldsymbol{H}_{0}(\operatorname{curl} ; \Omega)=\boldsymbol{Z}_{0}(\mathbf{I}, \Omega) \oplus \mathcal{N}(\Omega),
$$

where we abbreviated $\mathcal{N}(\Omega):=\boldsymbol{H}_{0}(\operatorname{curl} 0 ; \Omega)$. Merging the variational problem (5.1) and (5.6) we arrive at the following. Seek $\mathbf{e}_{\perp} \in \boldsymbol{Z}_{0}(\mathbf{I}, \Omega)$, $\mathbf{e}_{0} \in \mathcal{N}(\Omega)$ such that

$$
\begin{aligned}
\left(\boldsymbol{\mu}^{-1} \operatorname{curl} \mathbf{e}_{\perp}, \operatorname{curl} \mathbf{e}_{\perp}^{\prime}\right)_{\boldsymbol{L}^{2}(\Omega)} & \\
-\omega^{2}\left(\boldsymbol{\epsilon} \mathbf{e}_{\perp}, \mathbf{e}_{\perp}^{\prime}\right)_{\boldsymbol{L}^{2}(\Omega)}-\omega^{2}\left(\boldsymbol{\epsilon} \mathbf{e}_{0}, \mathbf{e}_{\perp}^{\prime}\right)_{\boldsymbol{L}^{2}(\Omega)} & =-i \omega\left(\mathbf{j}, \mathbf{e}_{\perp}^{\prime}\right)_{\boldsymbol{L}^{2}(\Omega)}, \\
\omega^{2}\left(\mathbf{e}_{\perp}, \mathbf{e}_{0}^{\prime}\right)_{\boldsymbol{L}^{2}(\Omega)}+\omega^{2}\left(\mathbf{e}_{0}, \mathbf{e}_{0}^{\prime}\right)_{\boldsymbol{L}^{2}(\Omega)} & =i \omega\left(\mathbf{j}, \mathbf{e}_{0}^{\prime}\right)_{\boldsymbol{L}^{2}(\Omega)}
\end{aligned}
$$

for all $\mathbf{e}_{\perp}^{\prime} \in \boldsymbol{Z}_{0}(\boldsymbol{\epsilon}, \Omega), \mathbf{e}_{0} \in \mathcal{N}(\Omega)$. It is important to notice that the sign of the second equation has been flipped. Therefore, in the sesqui-linear form corresponding to the above split problem, the 'principal' diagonal parts have the same (positive) sign. That they are really principal is rigorously confirmed by the following lemma. To state it, we introduce the $\boldsymbol{L}^{2}(\Omega)$ orthogonal projections $\mathrm{P}^{\perp}: \boldsymbol{H}_{0}(\operatorname{curl} ; \Omega) \mapsto \boldsymbol{Z}_{0}(\mathbf{I}, \Omega), \mathrm{P}^{0}: \boldsymbol{H}_{0}(\operatorname{curl} ; \Omega) \mapsto$ $\mathcal{N}(\Omega), \mathrm{P}^{\perp}+\mathrm{P}^{0}=\mathrm{Id}$, associated with (5.6).

Lemma 5.1. The sesqui-linear form $k: \boldsymbol{H}_{0}(\operatorname{curl} ; \Omega) \times \boldsymbol{H}_{0}(\operatorname{curl} ; \Omega) \mapsto \mathbb{C}$,

$$
k(\mathbf{u}, \mathbf{v}):=\left(\boldsymbol{\epsilon} \mathrm{P}^{\perp} \mathbf{u}, \mathrm{P}^{\perp} \mathbf{v}\right)_{\boldsymbol{L}^{2}(\Omega)}-\left(\boldsymbol{\epsilon} \mathrm{P}^{\perp} \mathbf{u}, \mathrm{P}^{0} \mathbf{v}\right)_{\boldsymbol{L}^{2}(\Omega)}+\left(\boldsymbol{\epsilon} \mathrm{P}^{0} \mathbf{u}, \mathrm{P}^{\perp} \mathbf{v}\right)_{\boldsymbol{L}^{2}(\Omega)},
$$

is compact.

Proof. We can split $k=k_{1}-k_{2}+k_{3}$,

$$
\begin{aligned}
& k_{1}(\mathbf{u}, \mathbf{v}):=\left(\epsilon \mathrm{P}^{\perp} \mathbf{u}, \mathrm{P}^{\perp} \mathbf{v}\right) \boldsymbol{L}^{2}(\Omega) \\
& k_{2}(\mathbf{u}, \mathbf{v}):=\left(\epsilon \mathrm{P}^{\perp} \mathbf{u}, \mathrm{P}^{0} \mathbf{v}\right) \boldsymbol{L}^{2}(\Omega) \\
& k_{3}(\mathbf{u}, \mathbf{v}):=\left(\epsilon \mathrm{P}^{0} \mathbf{u}, \mathrm{P}^{\perp} \mathbf{v}\right)_{L^{2}(\Omega)}
\end{aligned}
$$

and deal with the individual terms separately. First, note that $k_{3}(\mathbf{u}, \mathbf{v})=$ $\overline{k_{2}(\mathbf{v}, \mathbf{u})}$. Therefore, compactness of $k_{3}$ will be implied by that of $k_{2}$. Recall that a sesqui-linear form is defined to be compact if this holds for the associated operator.

Write $K_{1}: \boldsymbol{H}_{0}(\operatorname{curl} ; \Omega) \mapsto \boldsymbol{H}_{0}(\operatorname{curl} ; \Omega)^{\prime}$ for the bounded linear operator spawned by $k_{1}$. We find a $C>0$ only depending on $\epsilon$ such that

$$
\left\|K_{1} \mathbf{u}\right\|_{\boldsymbol{H}_{0}(\mathbf{c u r l} ; \Omega)^{\prime}}=\sup _{\mathbf{v} \in \boldsymbol{H}_{0}(\mathbf{c u r l} ; \Omega)} \frac{\left|k_{1}(\mathbf{u}, \mathbf{v})\right|}{\|\mathbf{v}\|_{\boldsymbol{H}(\mathbf{c u r l} ; \Omega)}} \leq C\left\|\mathrm{P}^{\perp} \mathbf{u}\right\|_{\boldsymbol{L}^{2}(\Omega)} .
$$


Then we can appeal to the compact embedding $\boldsymbol{Z}_{0}(\mathbf{I}, \Omega) \hookrightarrow \boldsymbol{L}^{2}(\Omega)$ stated in Corollary 4.3 to verify the compactness of $K_{1}$. Exactly the same argument settles the compactness of $K_{2}$.

However, what we discretize is (5.1) and not a split problem. In order to express the splitting idea in terms of $a(\cdot, \cdot)$, we resort to the isometric involution $\mathrm{X}: \boldsymbol{H}_{0}(\operatorname{curl} ; \Omega) \mapsto \boldsymbol{H}_{0}(\operatorname{curl} ; \Omega), \mathrm{X}:=\mathrm{P}^{\perp}-\mathrm{P}^{0}$. It naturally emerges from the flipping of signs that accompanied the formulation of the split variational problem. Thus we get

$$
\begin{aligned}
a(\mathbf{u}, \mathbf{X} \mathbf{v})=\left(\boldsymbol{\mu}^{-1} \operatorname{curl} \mathrm{P}^{\perp} \mathbf{u}, \operatorname{curl} \mathrm{P}^{\perp} \mathbf{v}\right) & \boldsymbol{L}^{2}(\Omega) \\
& +\omega^{2}\left(\boldsymbol{\epsilon} \mathrm{P}^{0} \mathbf{u}, \mathrm{P}^{0} \mathbf{v}\right) \boldsymbol{L}^{2}(\Omega) \\
& -k(\mathbf{u}, \mathbf{v}) .
\end{aligned}
$$

In view of Lemma 5.1, the properties of metric tensors, and Corollary 4.4, this implies a generalized Garding inequality, in the sense that, for all $\mathbf{u} \in$ $\boldsymbol{H}_{0}(\operatorname{curl} ; \Omega)$ and $C_{G}=C_{G}(\Omega, \boldsymbol{\epsilon}, \boldsymbol{\mu})>0$,

$$
|a(\mathbf{u}, \mathbf{X u})+k(\mathbf{u}, \mathbf{u})| \geq C_{G}\|\mathbf{u}\|_{\boldsymbol{H}(\mathbf{c u r l} ; \Omega)}^{2} .
$$

Standard theory based on the Fredholm alternative gives existence of continuous solutions.

Theorem 5.2. (Existence and uniqueness of continuous solution) Under Assumption 1 a solution of the variational problem (5.1) exists for every admissible right-hand side.

Existence and uniqueness of solutions of linear variational problems guarantee the following inf-sup condition for the associated bilinear form. There exists $C_{S}>0$ such that

$$
\sup _{\mathbf{v} \in \boldsymbol{H}_{0}(\mathbf{c u r l} ; \Omega)} \frac{|a(\mathbf{u}, \mathbf{v})|}{\|\mathbf{v}\|_{\boldsymbol{H}(\mathbf{c u r l} ; \Omega)}} \geq C_{S}\|\mathbf{u}\|_{\boldsymbol{H}(\mathbf{c u r l} ; \Omega)} \quad \forall \mathbf{u} \in \boldsymbol{H}_{0}(\mathbf{c u r l} ; \Omega) .
$$

\subsection{A priori error estimates}

We know from Babuška's theory (Babuška 1971) that we have to establish a discrete analogue of (5.9), that is,

$$
\sup _{\mathbf{v}_{h} \in \mathcal{W}_{p, 0}^{1}\left(\Omega_{h}\right)} \frac{\left|a\left(\mathbf{u}_{h}, \mathbf{v}_{h}\right)\right|}{\left\|\mathbf{v}_{h}\right\|_{\boldsymbol{H}(\mathbf{c u r l} ; \Omega)}} \geq C_{D}\left\|\mathbf{u}_{h}\right\|_{\boldsymbol{H}(\mathbf{c u r l} ; \Omega)} \quad \forall \mathbf{u}_{h} \in \mathcal{W}_{p, 0}^{1}\left(\Omega_{h}\right),
$$

because it permits us to conclude, for any $\mathbf{w}_{h} \in \mathcal{W}_{p}^{1}\left(\Omega_{h}\right)$,

$$
\begin{aligned}
\left\|\mathbf{e}-\mathbf{e}_{h}\right\|_{\boldsymbol{H}(\operatorname{curl} ; \Omega)} & \leq\left\|\mathbf{e}-\mathbf{w}_{h}\right\|_{\boldsymbol{H}(\operatorname{curl} ; \Omega)}+\left\|\mathbf{w}_{h}-\mathbf{e}_{h}\right\|_{\boldsymbol{H}(\mathbf{c u r l} ; \Omega)} \\
& \leq\left\|\mathbf{e}-\mathbf{w}_{h}\right\|_{\boldsymbol{H}(\operatorname{curl} ; \Omega)}+C_{D}^{-1} \sup _{\mathbf{v}_{h} \in \mathcal{W}_{p, 0}^{1}\left(\Omega_{h}\right)} \frac{\left|a\left(\mathbf{e}_{h}-\mathbf{w}_{h}, \mathbf{v}_{h}\right)\right|}{\left\|\mathbf{v}_{h}\right\|_{\boldsymbol{H}(\operatorname{curl} ; \Omega)}}
\end{aligned}
$$




$$
\begin{aligned}
& \leq\left\|\mathbf{e}-\mathbf{w}_{h}\right\|_{\boldsymbol{H}(\mathbf{c u r l} ; \Omega)}+C_{D}^{-1} \sup _{\mathbf{v}_{h} \in \mathcal{W}_{p, 0}^{1}\left(\Omega_{h}\right)} \frac{\left|a\left(\mathbf{e}-\mathbf{w}_{h}, \mathbf{v}_{h}\right)\right|}{\left\|\mathbf{v}_{h}\right\|_{\boldsymbol{H}(\mathbf{c u r l} ; \Omega)}} \\
& \leq\left(1+C_{D}^{-1} C_{A}\right)\left\|\mathbf{e}-\mathbf{w}_{h}\right\|_{\boldsymbol{H}(\mathbf{c u r l} ; \Omega)},
\end{aligned}
$$

as we have Galerkin orthogonality $a\left(\mathbf{e}-\mathbf{e}_{h}, \mathbf{v}_{h}\right)=0$ for all $\mathbf{v}_{h} \in \mathcal{W}_{p, 0}^{1}\left(\Omega_{h}\right)$. Here,

$$
C_{A}:=\max \left\{\left\|\boldsymbol{\mu}^{-1}\right\|_{L^{\infty}(\Omega)},\|\boldsymbol{\epsilon}\|_{L^{\infty}(\Omega)}\right\}
$$

is a bound for the norm of the bilinear form $a(\cdot, \cdot)$.

As a first step towards a discrete inf-sup condition, we have to find a suitable candidate for $\mathbf{v}$ in (5.9). To that end, for $\mathbf{u} \in \boldsymbol{H}_{0}(\mathbf{c u r l} ; \Omega)$ fix Su $\in \boldsymbol{H}_{0}(\operatorname{curl} ; \Omega)$ by

$$
a\left(\mathbf{u}^{\prime}, \mathbf{S u}\right)=k\left(\mathbf{u}, \mathbf{u}^{\prime}\right) \quad \forall \mathbf{u}^{\prime} \in \boldsymbol{H}_{0}(\operatorname{curl} ; \Omega) .
$$

This defines a bounded linear operator, S: $\boldsymbol{H}_{0}(\operatorname{curl} ; \Omega) \mapsto \boldsymbol{H}_{0}(\operatorname{curl} ; \Omega)$, thanks to Theorem 5.2.

Lemma 5.3. The operator $\mathrm{S}: \boldsymbol{H}_{0}(\operatorname{curl} ; \Omega) \mapsto \boldsymbol{H}_{0}(\operatorname{curl} ; \Omega)$ is compact.

Proof. By (5.9) we find

$$
\|\mathbf{S u}\|_{\boldsymbol{H}(\mathbf{c u r l} ; \Omega)} \leq C \sup _{\mathbf{v} \in \boldsymbol{H}_{0}(\mathbf{c u r l} ; \Omega)} \frac{|a(\mathbf{S u}, \mathbf{v})|}{\|\mathbf{v}\|_{\boldsymbol{H}(\mathbf{c u r l} ; \Omega)}} \leq C \sup _{\mathbf{v} \in \boldsymbol{H}_{0}(\mathbf{c u r l} ; \Omega)} \frac{|k(\mathbf{u}, \mathbf{v})|}{\|\mathbf{v}\|_{\boldsymbol{H}(\mathbf{c u r l} ; \Omega)}}
$$

and the compactness of $\mathrm{S}$ follows from that of $k$.

Using the definition of $\mathbf{S}$ it is immediate that, for all $\mathbf{u} \in \boldsymbol{H}_{0}(\operatorname{curl} ; \Omega)$,

$$
a(\mathbf{u},(\mathbf{X}+\mathbf{S}) \mathbf{u})=a(\mathbf{u}, \mathbf{X u})+k(\mathbf{u}, \mathbf{u}) \geq C_{G}\|\mathbf{u}\|_{\boldsymbol{H}(\mathbf{c u r l} ; \Omega)}^{2} .
$$

The choice $\mathbf{v}:=(\mathrm{X}+\mathrm{S}) \mathbf{u}$ will make (5.9) hold with $C_{S}=C_{G}$. The challenge is that $(X+S) \mathbf{u}_{h}$ will not be a finite element function even for $\mathbf{u}_{h} \in \mathcal{W}_{p, 0}^{1}\left(\Omega_{h}\right)$. It will be necessary to project it back to the finite element space by applying a suitable continuous projection operator $\widetilde{\mathrm{F}}_{h}: \boldsymbol{H}_{0}(\operatorname{curl} ; \Omega) \mapsto \mathcal{W}_{p, 0}^{1}\left(\Omega_{h}\right)$. Then, for an arbitrary $\mathbf{u}_{h} \in \mathcal{W}_{p, 0}^{1}\left(\Omega_{h}\right)$, we can hope that $\mathbf{v}_{h}:=\widetilde{\mathrm{F}}_{h}(\mathrm{X}+\mathrm{S}) \mathbf{u}_{h}$ is an appropriate choice for $\mathbf{v}_{h}$ in (5.10). Making use of (5.11), we see that

$$
\begin{aligned}
& \left|a\left(\mathbf{u}_{h}, \mathbf{v}_{h}\right)\right| \\
& \quad=\left|a\left(\mathbf{u}_{h},(\mathbf{X}+\mathrm{S}) \mathbf{u}_{h}\right)-a\left(\mathbf{u}_{h},\left(\mathrm{Id}-\widetilde{\mathrm{F}}_{h}\right)(\mathbf{X}+\mathrm{S}) \mathbf{u}_{h}\right)\right| \\
& \quad \geq C_{G}\left\|\mathbf{u}_{h}\right\|_{\boldsymbol{H}(\mathbf{c u r l} ; \Omega)}^{2}-C_{A}\left\|\mathbf{u}_{h}\right\|_{\boldsymbol{H}(\mathbf{c u r l} ; \Omega)}\left\|\left(\mathrm{Id}-\widetilde{\mathrm{F}}_{h}\right)(\mathbf{X}+\mathrm{S}) \mathbf{u}_{h}\right\|_{\boldsymbol{H}(\mathbf{c u r l} ; \Omega)} .
\end{aligned}
$$

Obviously, the projector $\widetilde{\mathrm{F}}_{h}$ has to effect uniform convergence $\left(\mathrm{Id}-\widetilde{\mathrm{F}}_{h}\right)(\mathrm{X}+\mathrm{S})$ $\rightarrow 0$ in $\boldsymbol{H}_{0}(\mathbf{c u r l} ; \Omega)$. Again, let us compare the situation with the boundary value problem for the Helmholtz equation. There the choice $X=\operatorname{Id}$ is possible, but we also have to choose a projector. This is easy, because the compactness of $\mathbf{S}$ converts pointwise convergence into uniform convergence. 
Lemma 5.4. If $\widetilde{\mathrm{F}}_{h} \rightarrow$ Id pointwise in $\boldsymbol{H}_{0}(\mathbf{c u r l} ; \Omega)$, then there is a function $\epsilon:] 0 ; \infty\left[\rightarrow \mathbb{R}^{+}\right.$with $\lim _{h \rightarrow 0} \epsilon(h)=0$ such that

$$
\left\|\left(\mathrm{Id}-\widetilde{\mathrm{F}}_{h}\right) \operatorname{Su}\right\|_{\boldsymbol{H}(\mathbf{c u r l} ; \Omega)} \leq \epsilon(h)\|\mathbf{u}\|_{\boldsymbol{H}(\mathbf{c u r l} ; \Omega)} \quad \forall \mathbf{u} \in \boldsymbol{H}_{0}(\operatorname{curl} ; \Omega) .
$$

Proof. A similar, even more general, statement is made in Kress (1989, Corollary 10.4).

This settles everything for the Helmholtz problem. However, the components of the Helmholtz decomposition of a discrete 1-form $\in \mathcal{W}_{p}^{1}\left(\Omega_{h}\right)$ do not necessarily belong to finite element spaces. Therefore, the projector $\widetilde{\mathrm{F}}_{h}$ will not necessarily act as identity on $\mathbf{X}\left(\mathcal{W}_{p}^{1}\left(\Omega_{h}\right)\right)$; we cannot escape estimates of the operator norm of $\left(\mathrm{Id}-\widetilde{\mathrm{F}}_{h}\right) \mathrm{X}$ on $\boldsymbol{H}_{0}(\mathbf{c u r l} ; \Omega)$ and have to show that it tends to zero as $h \rightarrow 0$.

Pondering these requirements, the Fortin projector (for $\boldsymbol{\epsilon}=\mathbf{I}$ ) from Section 4.2 looks promising. It can be used as the key ingredient of the projector $\widetilde{\mathrm{F}}_{h}$, which is chosen as

$$
\widetilde{\mathrm{F}}_{h}: \boldsymbol{H}_{0}(\operatorname{curl} ; \Omega) \mapsto \mathcal{W}_{p, 0}^{1}\left(\Omega_{h}\right), \quad \widetilde{\mathrm{F}}_{h}:=\mathrm{F}_{h} \mathrm{P}^{\perp}+\mathrm{Q}_{h}^{\mathcal{N}} \mathrm{P}^{0},
$$

where $Q_{h}^{\mathcal{N}}$ is the $L^{2}(\Omega)$-orthogonal projection $\mathcal{N}(\Omega) \mapsto \mathcal{N}\left(\Omega_{h}\right), \mathcal{N}\left(\Omega_{h}\right):=$ $\mathcal{W}_{p, 0}^{1}\left(\Omega_{h}\right) \cap \operatorname{Ker}(\mathbf{c u r l})$.

Lemma 5.5. We have pointwise convergence $\lim _{h \rightarrow 0}\left\|\mathbf{u}-\widetilde{\mathbf{F}}_{h} \mathbf{u}\right\|_{\boldsymbol{H}(\mathbf{c u r l} ; \Omega)}=0$ for any $\mathbf{u} \in \boldsymbol{H}_{0}(\operatorname{curl} ; \Omega)$.

Proof. From Theorem 4.8, for some $s>0$ and $C=C\left(\Omega, p, s, \rho\left(\Omega_{h}\right)\right)$,

$$
\left\|\left(\operatorname{Id}-\mathrm{F}_{h}\right) \mathrm{P}^{\perp} \mathbf{u}\right\|_{\boldsymbol{L}^{2}(\Omega)} \leq C h^{s}\|\operatorname{curl} \mathbf{u}\|_{\boldsymbol{L}^{2}(\Omega)} \rightarrow 0 \quad \text { for } h \rightarrow 0 .
$$

Moreover, as $h \rightarrow 0$ any $\mathbf{v} \in \mathcal{N}(\Omega)$ can be arbitrarily well approximated by a $\mathbf{v}_{h} \in \mathcal{N}\left(\Omega_{h}\right)$, which means $\left\|\mathbf{v}-Q_{h}^{\mathcal{N}} \mathbf{v}\right\|_{L^{2}(\Omega)} \rightarrow 0$ for $h \rightarrow 0$.

Combined with Lemma 5.5 this shows uniform convergence $\left(\mathrm{Id}-\widetilde{\mathrm{F}}_{h}\right) \mathrm{S} \rightarrow$ 0 in $\boldsymbol{H}_{0}(\mathbf{c u r l} ; \Omega)$. It turns out that the essential 'uniform convergence' $\left(\mathrm{Id}-\widetilde{\mathrm{F}}_{h}\right) \mathrm{X}_{\mid \mathcal{W}_{p}^{1}\left(\Omega_{h}\right)} \rightarrow 0$ also holds.

Lemma 5.6. There is an $s>0$ and $C_{*}=C_{*}\left(\Omega, p, s, \rho\left(\Omega_{h}\right)\right)>0$ such that

$$
\left\|\left(\operatorname{Id}-\widetilde{\mathrm{F}}_{h}\right) \mathbf{X}_{\mathbf{u}_{h}}\right\|_{\boldsymbol{H}(\mathbf{c u r l} ; \Omega)} \leq C_{*} h^{s}\left\|\operatorname{curl} \mathbf{u}_{h}\right\|_{\boldsymbol{L}^{2}(\Omega)} \quad \forall \mathbf{u}_{h} \in \mathcal{W}_{p, 0}^{1}\left(\Omega_{h}\right) .
$$

Proof. The key to the proof is to employ the $\boldsymbol{L}^{2}(\Omega)$-orthogonal discrete Helmholtz decomposition

$$
\mathcal{W}_{p, 0}^{1}\left(\Omega_{h}\right)=Z_{h, 0}\left(\mathbf{I}, \Omega_{h}\right) \oplus \mathcal{N}\left(\Omega_{h}\right),
$$


and the associated orthogonal projections $\mathrm{P}_{h}^{\perp}: \mathcal{W}_{p, 0}^{1}\left(\Omega_{h}\right) \mapsto \boldsymbol{Z}_{h, 0}\left(\mathbf{I}, \Omega_{h}\right)$, $\mathrm{P}_{h}^{0}: \mathcal{W}_{p, 0}^{1}\left(\Omega_{h}\right) \mapsto \mathcal{N}\left(\Omega_{h}\right), \mathrm{P}_{h}^{\perp}+\mathrm{P}_{h}^{0}=\mathrm{Id}$. As $\mathcal{N}\left(\Omega_{h}\right) \subset \mathcal{N}(\Omega)$ it is immediate that

$$
\mathrm{P}^{0} \circ \mathrm{P}_{h}^{0}=\mathrm{P}_{h}^{0} \quad \text { and } \quad \mathrm{P}^{\perp} \circ \mathrm{P}_{h}^{0}=0 .
$$

Alert readers will have noticed that $\mathrm{Q}_{h}^{\mathcal{N}}=\mathrm{P}_{h}^{0}$ and $\mathrm{P}^{\perp}=\mathrm{H}_{\mathbf{I}}$ with the Hodge mapping $\mathrm{H}_{\boldsymbol{\epsilon}}$ from Section 4.2 (for $\boldsymbol{\epsilon}=\mathbf{I}$ ). Thus, using the projection properties, in particular $\widetilde{\mathrm{F}}_{h} \circ \mathrm{P}_{h}^{0}=\mathrm{Id}$, we can rewrite

$$
\begin{aligned}
\left(\mathrm{Id}-\widetilde{\mathrm{F}}_{h}\right) \mathrm{X} & =\left(\mathrm{P}^{\perp}-\mathrm{F}_{h} \mathrm{P}^{\perp}+\mathrm{P}^{0}-\mathrm{Q}_{h}^{\mathcal{N}} \mathrm{P}^{0}\right)\left(\mathrm{P}^{\perp}-\mathrm{P}^{0}\right)\left(\mathrm{P}_{h}^{\perp}+\mathrm{P}_{h}^{0}\right) \\
& =\left(\mathrm{Id}-\mathrm{F}_{h}\right) \mathrm{P}^{\perp} \mathrm{P}_{h}^{\perp}-\left(\mathrm{Id}-\mathrm{P}_{h}^{0}\right) \mathrm{P}^{0} \mathrm{P}_{h}^{\perp} \\
& =\left(\mathrm{Id}-\mathrm{F}_{h}\right) \mathrm{P}^{\perp} \mathrm{P}_{h}^{\perp}-\left(\mathrm{Id}-\mathrm{P}_{h}^{0}\right) \mathrm{P}^{0}\left(\mathrm{P}_{h}^{\perp}-\mathrm{P}^{\perp}\right) \mathrm{P}_{h}^{\perp} \\
& =\left(\mathrm{H}_{\mathbf{I}}-\mathrm{Id}\right) \mathrm{P}_{h}^{\perp}-\left(\mathrm{Id}-\mathrm{P}_{h}^{0}\right) \mathrm{P}^{0}\left(\mathrm{Id}-\mathrm{H}_{\mathbf{I}}\right) \mathrm{P}_{h}^{\perp} \\
& =\left(\mathrm{Id}+\left(\mathrm{Id}-\mathrm{P}_{h}^{0}\right) \mathrm{P}^{0}\right)\left(\mathrm{H}_{\mathbf{I}}-\mathrm{Id}\right) \mathrm{P}_{h}^{\perp} .
\end{aligned}
$$

Now it remains to use Lemma 4.5.

Summing up, the two previous lemmas give us

$$
\left\|\left(\operatorname{Id}-\widetilde{\mathrm{F}}_{h}\right)(\mathrm{X}+\mathrm{S}) \mathbf{u}_{h}\right\|_{\boldsymbol{H}(\mathbf{c u r l} ; \Omega)} \leq \epsilon(h)+C_{*} h^{s}\left\|\mathbf{u}_{h}\right\|_{\boldsymbol{H}(\mathbf{c u r l} ; \Omega)} .
$$

Thus, for $h$ sufficiently small to ensure $1-C_{A}\left(\epsilon(h)+C_{*} h^{s}\right) / C_{G}>\frac{1}{2}$, we have the discrete inf-sup condition (5.10). This yields the main result.

Theorem 5.7. Provided that Assumption 1 holds, there exists $h^{*}>0$ depending on the parameters of the continuous problem and the shape regularity measure of the triangulation, such that a unique solution $\mathbf{e}_{h}$ of the discrete problem (5.2) exists, provided that $h<h_{*}$. It provides an asymptotically optimal approximation to the continuous solution e of (5.1) in the sense of (5.3).

It must be emphasized that the result is asymptotic in nature. Computational resources hardly ever permit us to use very fine meshes on threedimensional domains. Hence, the relevance of the statement of Theorem 5.7 for a concrete problem and mesh is not clear.

Remark 16. Even if we accept asymptotic optimality as an essential property, actual rates of convergence will still hinge on the smoothness of the solution e. The comprehensive investigations in Costabel et al. (1999) and Costabel and Dauge (1998) send a daunting message: even for smooth $\boldsymbol{\epsilon}, \boldsymbol{\mu}$, and $\mathbf{j}$, the solution for e might not belong to $\boldsymbol{H}^{1}(\Omega)$ if the boundary possesses re-entrant edges/corners. Only a regularity of the form $\mathbf{e} \in \boldsymbol{H}^{\frac{1}{2}+\epsilon}(\Omega)$, $\epsilon>0$, can be expected. If there are jumps in $\epsilon$ the situation can be even worse, reducing the regularity of $\mathbf{e}$ down to a mere $\mathbf{e} \in \boldsymbol{H}^{\epsilon}(\Omega), \epsilon>0$. Therefore, in Section 3.6 we have taken great pains to get interpolation estimates 
in rather weak fractional Sobolev norms. Despite the effort, the (theoretical) rates of convergence of the $h$-version of discrete differential forms can be abysmally poor.

Yet the results of Costabel et al. (1999) and Costabel and Dauge (1998) also imply (cf. Costabel and Dauge (2002, Section 6)) that, for analytic $\boldsymbol{\epsilon}, \boldsymbol{\mu}, \mathbf{j}$, the solution e can be decomposed according to $\mathbf{e}=\boldsymbol{\Psi}+\operatorname{grad} \varphi$, where both $\boldsymbol{\Psi}$ and $\varphi$ belong to countably normed spaces defined by the intersection of weighted Sobolev spaces. The weight functions are powers of distances from the geometric singularities of the boundary.

Again, it is wise to remember the guideline of Section 3.2. Barring highly irregular (in practical terms) coefficients and data, the so-called $h p$-version of finite elements (Babuška and Suri 1994, Schwab 1998, Khoromskij and Melenk 2001) can achieve exponential order of convergence of approximate solutions of second-order elliptic problems by judiciously combining local mesh refinement and local adjustments of polynomials degrees in $\mathbf{p}$. It is prerequisite that the solution belongs to those special countably normed spaces mentioned above. Thus we realize that conditions for the exponential convergence of a $h p$-version of discrete differentials forms are also met in the cases of the Maxwell source problem and the Maxwell eigenvalue problem. This justifies the following prediction, though a complete theory is still missing: The (adaptive) hp-version of discrete differential forms will provide the most efficient discretization for the Maxwell source problems/Maxwell eigenvalue problem, provided that the coefficients $\boldsymbol{\mu}, \boldsymbol{\epsilon}$ and excitation $\mathbf{j}$ are (piecewise) analytic.

Remark 17. Of course, the constant in (5.3) will blow up as $\omega$ approaches a Maxwell eigenvalue. We may hope to avoid this in the case of complex $\boldsymbol{\epsilon}$, i.e., for conducting media or by imposing absorbing boundary conditions ( $c f$. Remark 2) instead of PEC. This seems to cure the problem. However, investigations of the behaviour of Lagrangian finite elements for the Helmholtz problem (5.4) disclosed that, for $h$-version finite elements, a blow-up of $C$ still occurs as $\omega$ increases (Ihlenburg 1998). This is the so-called pollution effect notorious for fixed degree FEM in acoustics. There is empirical evidence that discrete 1 -forms are also vulnerable to pollution. A partial remedy is the use of higher-order schemes. This provides another rationale for studying these carefully in Section 3.4.

\section{Bibliographical notes}

The first convergence analysis of the time-harmonic Maxwell's equations, when discretized by means of edge elements, was given by P. Monk (1992a). The above proof follows the lines of Buffa, Hiptmair, von Petersdorff and Schwab (2002, Section 4.1). In Monk and Demkowicz (2001) and Kirsch and Monk (2000, Section 4) it was shown that the analysis can also rely on 
discrete compactness ( $c f$. Section 4.4). The application of abstract theory, for nonlinear problems (Brezzi, Rappaz and Raviart 1980) to the Maxwell source problem, is studied in Boffi and Gastaldi (2001). Matching their potential, much effort has recently been put into the analysis and implementation of $h p$-FEM for discrete 1-forms (Ainsworth and Pinchedez 2001, Rachowicz and Demkowicz 2002, Vardapetyan and Demkowicz 1999).

\subsection{Duality estimates}

For the Maxwell source problem, we expect the error norm $\left\|\mathbf{e}-\mathbf{e}_{h}\right\|_{\boldsymbol{L}^{2}(\Omega)}$ to decrease faster than $\left\|\mathbf{e}-\mathbf{e}_{h}\right\|_{\boldsymbol{H}(\mathbf{c u r l} ; \Omega)}$ as $h \rightarrow 0$. In finite element theory, duality estimates, often referred to as Aubin-Nitsche tricks (Ciarlet 1978, Section 3.2), are an effective technique with which such questions are tackled. In light of the guideline stated in Section 3.2 and Remark 1, it is not surprising that they can be adapted to the Maxwell source problem.

Elliptic regularity plays a pivotal role in duality estimates. Therefore, we have to investigate the regularity of solutions of the Maxwell source problem. With reasonable effort this is only possible in the case $\boldsymbol{\mu}, \boldsymbol{\epsilon}=\mathbf{I}$ (for more general results see Costabel et al. (1999)), which will be the only one considered in this subsection.

Theorem 5.8. (A priori error estimate in $\boldsymbol{L}^{2}(\Omega)$-norm) If $\boldsymbol{\mu}, \boldsymbol{\epsilon}=\mathbf{I}$, $\mathbf{j} \in \boldsymbol{H}(\operatorname{div} ; \Omega)$, and Assumption 1 holds, there exists $s>1 / 2$ such that the solutions $\mathbf{e}$ and $\mathbf{e}_{h}$ of (5.1) and (5.2) satisfy

$$
\left\|\mathbf{e}-\mathbf{e}_{h}\right\|_{\boldsymbol{L}^{2}(\Omega)} \leq C h^{s}\left\|\mathbf{e}-\mathbf{e}_{h}\right\|_{\boldsymbol{H}(\mathbf{c u r l} ; \Omega)},
$$

with $C>0$ independent of the meshwidth $h$.

Proof. Small wonder that the splitting idea is successful again. We start with

$$
\mathbf{e}-\mathbf{e}_{h}=\delta \mathbf{e}^{\perp}+\delta \mathbf{e}^{0}, \quad \delta \mathbf{e}^{\perp} \in \boldsymbol{Z}_{0}(\mathbf{I}, \Omega), \delta \mathbf{e}^{0} \in \mathcal{N}(\Omega) .
$$

Both components will be estimated separately. A genuine duality technique is required for the first: define $\mathbf{g} \in \boldsymbol{Z}_{0}(\mathbf{I}, \Omega)$ as the solution of

$$
a(\mathbf{g}, \mathbf{v})=\left(\delta \mathbf{e}^{\perp}, \mathbf{v}\right)_{\boldsymbol{L}^{2}(\Omega)} \forall \mathbf{v} \in \boldsymbol{Z}_{0}(\mathbf{I}, \Omega) .
$$

This is legal due to Theorem 5.2. From Theorem 4.1 and $\operatorname{div} \mathbf{g}=0$ we learn that $\mathbf{g} \in \boldsymbol{H}^{s}(\Omega)$ for some $\frac{1}{2}<s \leq 1$, and that

$$
\|\mathbf{g}\|_{\boldsymbol{H}^{s}(\Omega)} \leq C\|\mathbf{g}\|_{\boldsymbol{H}(\mathbf{c u r l} ; \Omega)}
$$

Moreover, (5.12) means that $\mathbf{g}$ satisfies, in the sense of distributions,

$$
\text { curlcurlg }-\omega^{2} \mathbf{g}=\delta \mathbf{e}^{\perp} \quad \text { in } \Omega .
$$

Thus, $\mathbf{w}:=$ curl g fulfils curl $\mathbf{w}=\delta \mathbf{e}^{\perp}+\omega^{2} \mathbf{g} \in \boldsymbol{L}^{2}(\Omega), \operatorname{div} \mathbf{w}=0$, and 
$\gamma_{\mathbf{n}} \mathbf{w}=0$. Using Lemma 4.2, we infer that $\mathbf{w} \in \boldsymbol{H}^{s}(\Omega)$ for some $\frac{1}{2}<s \leq 1$ and that

$$
\|\mathbf{w}\|_{\boldsymbol{H}^{s}(\Omega)} \leq C\|\mathbf{w}\|_{H(\operatorname{curl} ; \Omega)} \leq C\left(\|\mathbf{g}\|_{\boldsymbol{H}(\operatorname{curl} ; \Omega)}+\left\|\delta \mathbf{e}^{\perp}\right\|_{\boldsymbol{L}^{2}(\Omega)}\right) .
$$

This $s$ may be different from the one above. In what follows we keep the symbol $s$ to designate whichever is the smaller. Using (5.9) and (5.12) we obtain

$$
\|\mathbf{g}\|_{\boldsymbol{H}(\mathbf{c u r l} ; \Omega)} \leq C_{S}^{-1}\left\|\delta \mathbf{e}^{\perp}\right\|_{\boldsymbol{L}^{2}(\Omega)} .
$$

Combined with the previous estimates, this yields, with $C=C(\Omega)>0$,

$$
\|\mathbf{g}\|_{\boldsymbol{H}^{s}(\Omega)}+\|\operatorname{curl} \mathbf{g}\|_{\boldsymbol{H}^{s}(\Omega)} \leq C\left\|\delta \mathbf{e}^{\perp}\right\|_{\boldsymbol{L}^{2}(\Omega)} .
$$

Armed with these estimates, and Galerkin orthogonality $a\left(\mathbf{e}-\mathbf{e}_{h}, \mathbf{v}_{h}\right)=0$ for all $\mathbf{v}_{h} \in \mathcal{W}_{p, 0}^{1}\left(\Omega_{h}\right)$, we obtain

$$
\begin{aligned}
\left\|\delta \mathbf{e}^{\perp}\right\|_{\boldsymbol{L}^{2}(\Omega)}^{2} & =a\left(\mathbf{g}, \delta \mathbf{e}^{\perp}\right)=a\left(\mathbf{g}, \mathbf{e}-\mathbf{e}_{h}\right)=a\left(\mathbf{g}-\Pi_{p}^{1} \mathbf{g}, \delta \mathbf{e}\right) \\
& \leq C_{A}\left\|\mathbf{g}-\Pi_{p}^{1} \mathbf{g}\right\|_{\boldsymbol{H}(\mathbf{c u r l} ; \Omega)}\left\|\mathbf{e}-\mathbf{e}_{h}\right\|_{\boldsymbol{H}(\mathbf{c u r l} ; \Omega)} .
\end{aligned}
$$

We also used the fact that $\left(\mathbf{g}, \delta \mathbf{e}^{0}\right)_{\mathbf{L}^{2}(\Omega)}=0$ and hence, by Lemma 3.13 and (5.13), $\mathbf{g}$ is sufficiently smooth to ensure that moment-based local interpolation operators be well defined. Next, we rely on (5.14), Theorem 3.14 and Corollary 3.17 and get, with a positive constant $C=C\left(\Omega, p, s, \rho\left(\Omega_{h}\right)\right)$,

$$
\left\|\mathbf{g}-\Pi_{p}^{1} \mathbf{g}\right\|_{\boldsymbol{H}(\mathbf{c u r l} ; \Omega)} \leq C h^{s}\left\|\delta \mathbf{e}^{\perp}\right\|_{L^{2}(\Omega)} .
$$

The last two inequalities give the assertion for $\delta \mathbf{e}^{\perp}$.

In order to tackle $\delta \mathbf{e}^{0}$, we use

$$
-\omega^{2}\left(\delta \mathbf{e}^{0}, \mathbf{z}_{h}\right)_{\boldsymbol{L}^{2}(\Omega)}=a\left(\delta \mathbf{e}^{0}, \mathbf{z}_{h}\right)=0 \quad \forall \mathbf{z}_{h} \in \mathcal{N}\left(\Omega_{h}\right),
$$

which is a consequence of Galerkin orthogonality. For the time being, let us assume that $\left.\Pi_{p}^{1}\right) \delta \mathbf{e}^{0}$ is well defined. Then, by virtue of the commuting diagram property (3.12) this involves

$$
\left\|\delta \mathbf{e}^{0}\right\|_{\boldsymbol{L}^{2}(\Omega)} \leq\left\|\left(\operatorname{Id}-\Pi_{p}^{1}\right) \delta \mathbf{e}^{0}\right\|_{\boldsymbol{L}^{2}(\Omega)} .
$$

The observation $\delta \mathbf{e}^{\perp}=\mathrm{P}^{\perp}\left(\mathbf{e}-\mathbf{e}_{h}\right)$ confirms the identity

$$
\begin{aligned}
\left(\operatorname{Id}-\Pi_{p}^{1}\right) \delta \mathbf{e}^{0} & =\left(\operatorname{Id}-\Pi_{p}^{1}\right)\left(\mathrm{P}^{\perp}+\mathrm{P}^{0}\right) \mathbf{e}-\left(\operatorname{Id}-\Pi_{p}^{1}\right) \delta \mathbf{e}^{\perp} \\
& =\left(\operatorname{Id}-\Pi_{p}^{1}\right) \mathrm{P}^{0} \mathbf{e}+\left(\operatorname{Id}-\Pi_{p}^{1}\right) \mathrm{P}^{\perp} \mathbf{e}_{h} .
\end{aligned}
$$

The second term is amenable to the policy pursued in the proof of Lemma 4.5 and we get

$$
\left\|\left(\operatorname{Id}-\Pi_{p}^{1}\right) \mathrm{P}^{\perp} \mathbf{e}_{h}\right\|_{\boldsymbol{L}^{2}(\Omega)} \leq C h^{s}\left\|\operatorname{curl} \mathbf{e}_{h}\right\|_{\boldsymbol{L}^{2}(\Omega)} .
$$


To deal with the first term, we resort to Lemma 2.2, which gives a splitting $\mathrm{P}^{0} \mathbf{e}=\operatorname{grad} \varphi+\mathbf{q}, \varphi \in H_{0}^{1}(\Omega), \mathbf{q} \in \mathcal{H}^{2}(\Omega) \subset \boldsymbol{H}^{s}(\Omega)$. Note that $\varphi$ satisfies

$$
\omega(\operatorname{grad} \varphi, \operatorname{grad} \psi)_{\boldsymbol{L}^{2}(\Omega)}=i(\operatorname{div} \mathbf{j}, \psi)_{\boldsymbol{L}^{2}(\Omega)} \quad \forall \psi \in H_{0}^{1}(\Omega) .
$$

Remembering the connection between $s$ and the optimal lifting exponent for the Dirichlet problem for the Laplacian on $\Omega$ ( $c f$. Theorem 4.1), we see that $\operatorname{grad} \varphi \in \boldsymbol{H}^{s}(\Omega)$, too. In particular, now we know that $\Pi_{p}^{1} \delta \mathbf{e}^{0}$ really makes sense. Moreover, we can invoke Theorem 3.14, which gives

$$
\left\|\left(\mathrm{Id}-\Pi_{p}^{1}\right) \mathrm{P}^{0} \mathbf{e}\right\|_{\boldsymbol{L}^{2}(\Omega)} \leq C h^{s}\left(\left\|\mathrm{P}^{0} \mathbf{e}\right\|_{\boldsymbol{L}^{2}(\Omega)}+\|\operatorname{div} \mathbf{j}\|_{L^{2}(\Omega)}\right) .
$$

Combining all estimates, the proof is finished.

Remark 18. If $\Omega$ has a smooth boundary or is convex, which involves 2-regularity of the Dirichlet and Neumann problem for the Laplacian on $\Omega$, we can pick $s=1$ in the above theorem.

\section{Bibliographical notes}

Duality estimates for Maxwell source problems were pioneered by P. Monk, in Monk (1992b), and later refined in Monk (2001). They have been used in various contexts, for instance in Ciarlet, Jr, and Zou (1999, Section 4).

\subsection{Zero frequency limit}

Apart from problems faced for resonant $\omega$ the formulation (5.1) is also prone to instability when $\omega \rightarrow 0$. In the limit case $\omega=0$ a solution does not exist if $\mathbf{j} \notin \boldsymbol{H}(\operatorname{div} 0 ; \Omega)$. Even if this is satisfied, we can only expect uniqueness of $\mathbf{e}$ up to a contribution from $\boldsymbol{H}_{0}(\operatorname{curl} 0 ; \Omega)$.

Such stability problems are inherent in the derivation of (5.1) through the elimination of the magnetic field. Therefore, $\mathbf{e}$ is of a twin magnetic and electric nature. However, for $\omega=0$ the magnetic and electric field are completely decoupled: information on the magnetic field can no longer be conveyed through e.

We cannot help separating magnetic and electric quantities in the variational problem (5.1). A way to achieve this is the introduction of potentials. To avoid difficulties caused by the artificial electric walls, we will assume trivial topology of $\Omega$ in the remainder of this section.

Following (2.11), we write

$$
\mathbf{e}=-\operatorname{grad} v-i \omega \operatorname{curl} \mathbf{a}
$$

supplemented by a gauge condition to fix the potentials. For low frequencies the Coulomb gauge div $\mathbf{a}=0$ is most natural. Imposing the gauge constraint in weak form the variational problem is thus converted into the following 
saddle point problem. Seek $\mathbf{a} \in \boldsymbol{H}_{0}(\mathbf{c u r l} ; \Omega), v \in H_{0}^{1}(\Omega)$ such that

$$
\begin{aligned}
\left(\boldsymbol{\mu}^{-1} \operatorname{curl} \mathbf{a}, \operatorname{curl} \mathbf{a}^{\prime}\right)_{\boldsymbol{L}^{2}(\Omega)} & \\
-\omega^{2}\left(\boldsymbol{\epsilon} \mathbf{a}, \mathbf{a}^{\prime}\right)_{\boldsymbol{L}^{2}(\Omega)}+i \omega\left(\boldsymbol{\epsilon} \mathbf{a}^{\prime}, \operatorname{grad} v\right)_{\boldsymbol{L}^{2}(\Omega)} & =\left(\mathbf{j}, \mathbf{a}^{\prime}\right)_{\boldsymbol{L}^{2}(\Omega)}, \\
i \omega\left(\boldsymbol{\epsilon} \mathbf{a}, \operatorname{grad} v^{\prime}\right)_{\boldsymbol{L}^{2}(\Omega)} & =0,
\end{aligned}
$$

for all $\mathbf{a}^{\prime} \in \boldsymbol{H}_{0}(\mathbf{c u r l} ; \Omega), v^{\prime} \in H_{0}^{1}(\Omega)$. Here, $v$ can be regarded as a Lagrangian multiplier enforcing the divergence constraint on a (Kikuch 1987).

Theorem 5.9. If $\omega \geq 0$ complies with Assumption 1 , then the above saddle point problem has a unique solution $(\mathbf{a}, v)$.

Proof. As, evidently, $\mathbf{a} \in \boldsymbol{Z}_{0}(\boldsymbol{\epsilon}, \Omega)$, first consider the following restriction of the saddle point problem to $\boldsymbol{Z}_{0}(\boldsymbol{\epsilon}, \Omega)$. Seek $\mathbf{a} \in \boldsymbol{Z}_{0}(\boldsymbol{\epsilon}, \Omega)$ such that

$$
\left(\boldsymbol{\mu}^{-1} \operatorname{curl} \mathbf{a}, \operatorname{curl} \mathbf{a}^{\prime}\right)_{\boldsymbol{L}^{2}(\Omega)}-\omega^{2}\left(\boldsymbol{\epsilon} \mathbf{a}, \mathbf{a}^{\prime}\right)_{\boldsymbol{L}^{2}(\Omega)}=\left(\mathbf{j}, \mathbf{a}^{\prime}\right)_{\boldsymbol{L}^{2}(\Omega)}
$$

for all $\mathbf{a}^{\prime} \in \boldsymbol{Z}_{0}(\boldsymbol{\epsilon}, \Omega)$. By Corollary 4.3 we have coercivity, and Assumption 1 ensures uniqueness, which implies existence by the Fredholm alternative.

Secondly, observe that, thanks to $\operatorname{grad} H_{0}^{1}(\Omega) \subset \boldsymbol{H}_{0}(\mathbf{c u r l} ; \Omega)$,

$$
\sup _{\mathbf{v} \in \boldsymbol{H}_{0}(\operatorname{curl} ; \Omega)} \frac{(\boldsymbol{\epsilon}, \operatorname{grad} v)_{\boldsymbol{L}^{2}(\Omega)}}{\|\mathbf{v}\|_{\boldsymbol{H}(\operatorname{curl} ; \Omega)}} \geq \frac{(\boldsymbol{\epsilon} \operatorname{grad} v, \operatorname{grad} v)_{\boldsymbol{L}^{2}(\Omega)}}{\|\operatorname{grad} v\|_{\boldsymbol{H}(\operatorname{curl} ; \Omega)}} \geq C|v|_{H^{1}(\Omega)} .
$$

From this and the Poincaré-Friedrichs inequality in $H_{0}^{1}(\Omega)$, we conclude the uniqueness of $v$.

In a discrete setting based on discrete differential forms the nice properties of the continuous problem are preserved.

Theorem 5.10. For any $\omega \geq 0$ complying with Assumption 1 the saddle point problem discretized over $\mathcal{W}_{p, 0}^{1}\left(\Omega_{h}\right) \times \mathcal{W}_{p, 0}^{0}\left(\Omega_{h}\right)$ has a unique solution that converges quasioptimally provided that the meshwidth is sufficiently small.

Proof. Following the proof of the previous theorem, we first consider the following variational problem on $\boldsymbol{Z}_{h, 0}\left(\boldsymbol{\epsilon}, \Omega_{h}\right)$. Find $\mathbf{a}_{h} \in \boldsymbol{Z}_{h, 0}\left(\boldsymbol{\epsilon}, \Omega_{h}\right)$ with

$$
\left(\boldsymbol{\mu}^{-1} \operatorname{curl} \mathbf{a}_{h}, \operatorname{curl} \mathbf{a}_{h}^{\prime}\right)_{\boldsymbol{L}^{2}(\Omega)}-\omega^{2}\left(\boldsymbol{\epsilon} \mathbf{a}_{h}, \mathbf{a}_{h}^{\prime}\right)_{\boldsymbol{L}^{2}(\Omega)}=\left(\mathbf{j}, \mathbf{a}_{h}^{\prime}\right)_{\boldsymbol{L}^{2}(\Omega)}
$$

for all $\mathbf{a}_{h}^{\prime} \in \boldsymbol{Z}_{h, 0}\left(\boldsymbol{\epsilon}, \Omega_{h}\right)$. Using the techniques of Section 5.2 we get the assertion for $\mathbf{a}_{h}$. The details are left to the reader.

Then, thanks to $\operatorname{grad} \mathcal{W}_{p, 0}^{0}\left(\Omega_{h}\right) \subset \mathcal{W}_{p, 0}^{1}\left(\Omega_{h}\right)$ we can compute a unique $v_{h} \in \mathcal{W}_{p, 0}^{0}\left(\Omega_{h}\right)$ in a second step. The argument relies on the discrete version of (5.17). 


\section{Regularized formulations}

Using the discretization of the Maxwell eigenvalue problem studied in Section 4 we end up with a generalized eigenvalue problem for the coefficient vector $\overrightarrow{\mathbf{e}}$ describing the eigenmode. It has the form

$$
\mathrm{A}_{\boldsymbol{\mu}} \overrightarrow{\mathbf{e}}_{h}=\omega^{2} \mathrm{M}_{\boldsymbol{\epsilon}}^{1} \overrightarrow{\mathbf{e}}_{h}
$$

where both matrices are real, symmetric, $\mathrm{M}_{\boldsymbol{\epsilon}}^{1}$ is a positive definite mass matrix, but $A_{\boldsymbol{\mu}}$ is only positive semidefinite, beset with a large kernel. Usually, a few of the smallest nonzero eigenvalues and eigenfunctions of (4.7) are of interest. The vast numbers of degrees of freedom - inevitable for problems in three dimensions - force us to use iterative methods of inverse iteration type (Adam, Arbenz and Geus 1997, Section 7), most of which are crippled by the presence of a kernel. Thus, it becomes desirable to get rid of the kernel, of course, without affecting the approximation of eigenvectors and eigenvalues.

The kernel of $\mathrm{A}_{\boldsymbol{\mu}} \overrightarrow{\mathrm{e}}_{h}$ might also hamper the solution of the discretized Maxwell source problem. For instance, when applying Krylov subspace methods, e.g., MINRES (Hackbusch 1993), for its iterative solution, convergence will deteriorate, if there are large numbers of both negative and positive eigenvalues of the system matrix corresponding to (5.2). The presence of many negative eigenvalues may also hurt preconditioning techniques.

\subsection{Discrete regularization}

Any solution $\mathbf{e}_{h}$ of the Maxwell eigenvalue problem (4.7) will satisfy $\mathbf{e}_{h} \in$ $\boldsymbol{Z}_{h, 0}\left(\boldsymbol{\epsilon}, \Omega_{h}\right)$. Therefore all discrete eigenvalues and eigenfunctions of (4.7) can also be obtained from the following problem. Find $\mathbf{e} \in \mathcal{W}_{p, 0}^{1}\left(\Omega_{h}\right), \varphi_{h} \in$ $\mathcal{W}_{p, 0}^{0}\left(\Omega_{h}\right), \omega \neq 0$ such that, for some regularization parameter $s>0$,

$$
\begin{aligned}
\left(\boldsymbol{\mu}^{-1} \operatorname{curl} \mathbf{e}_{h}, \operatorname{curl} \mathbf{e}_{h}^{\prime}\right)_{\boldsymbol{L}^{2}(\Omega)}+\left(\boldsymbol{\epsilon} \mathbf{e}_{h}^{\prime}, \operatorname{grad} \varphi_{h}\right)_{\boldsymbol{L}^{2}(\Omega)} & =\omega^{2}\left(\boldsymbol{\epsilon} \mathbf{e}_{h}, \mathbf{e}_{h}^{\prime}\right)_{\boldsymbol{L}^{2}(\Omega)}, \\
\left(\boldsymbol{\epsilon} \mathbf{e}_{h}, \operatorname{grad} \varphi_{h}^{\prime}\right)_{\boldsymbol{L}^{2}(\Omega)}-\frac{1}{s} d\left(\varphi_{h}, \varphi_{h}^{\prime}\right) & =0,
\end{aligned}
$$

for all $\mathbf{e}_{h}^{\prime} \in \mathcal{W}_{p}^{1}\left(\Omega_{h}\right), \varphi_{h}^{\prime} \in \mathcal{W}_{p, 0}^{0}\left(\Omega_{h}\right)$. Note that, if $\mathbf{e}_{h}$ is a discrete eigenvalue of the original problem (4.7), the associated $\varphi_{h}$ turns out to be zero. Thus, $d(\cdot, \cdot)$ can be any $H^{1}(\Omega)$-continuous bilinear form on $\mathcal{W}_{p, 0}^{0}\left(\Omega_{h}\right)$. In matrix notation the above eigenvalue problem reads:

$$
\begin{aligned}
& \mathrm{A}_{\boldsymbol{\mu}} \overrightarrow{\mathbf{e}}_{h}+\mathrm{G} \vec{\varphi}_{h}=\omega^{2} \mathrm{M}_{\boldsymbol{\epsilon}}^{1} \overrightarrow{\mathbf{e}}_{h}, \\
& \mathrm{G}^{T} \overrightarrow{\mathbf{e}}_{h}-\frac{1}{s} \mathrm{D} \vec{\varphi}_{h}=0 .
\end{aligned}
$$

The case $d=0$ was already treated in Section 5.4. It leads to a saddle point problem, which is not the desirable outcome of regularization. Thus, the only sensible choices for $d(\cdot, \cdot)$ are symmetric, positive definite bilinear forms. ${ }^{8}$

${ }^{8}$ Remember that the eigenvalue problem can be tackled in an entirely real setting. 
Then D is invertible, and we can eliminate $\vec{\varphi}_{h}$, providing the symmetric generalized eigenvalue problem

$$
\left(\mathrm{A}_{\boldsymbol{\mu}}+s \mathrm{GD}^{-1} \mathrm{G}^{T}\right) \overrightarrow{\mathbf{e}}_{h}=\omega^{2} \mathrm{M}_{\epsilon}^{1} \overrightarrow{\mathbf{e}}_{h} .
$$

Lemma 6.1. If $\Omega$ has trivial topology, that is, its first and second Betti number vanish, then $\mathrm{A}_{\boldsymbol{\mu}}+s \mathrm{GD}^{-1} \mathrm{G}^{T}$ is positive definite. Its eigenvalues are bounded from below by $\min \left\{C_{1}^{1 / 2}, s C_{0}\|d\|^{-1 / 2}\right\}$, where $C_{1}$ is the constant of the discrete Poincaré-Friedrichs inequality from Theorem 4.7, and $C_{0}$ depends on $\epsilon$ and the constant of the Poincaré-Friedrichs inequality of $H_{0}^{1}(\Omega)$. Further,

$$
\|d\|:=\sup \left\{d\left(\varphi_{h}, \psi_{h}\right), \varphi_{h}, \psi_{h} \in \mathcal{W}_{p}^{0}\left(\Omega_{h}\right),\left\|\varphi_{h}\right\|_{H^{1}(\Omega)} \leq 1,\left\|\psi_{h}\right\|_{H^{1}(\Omega)} \leq 1\right\} .
$$

Proof. We can analyse the generalized saddle point problem behind the regularized formulation appealing to the 'ellipticity on kernel' (Brezzi and Fortin 1991, Chapter 2) expressed in Theorem 4.7, and the estimate

$$
\begin{aligned}
\sup _{\mathbf{v}_{h} \in \mathcal{W}_{p, 0}^{1}\left(\Omega_{h}\right)} \frac{\left(\boldsymbol{\epsilon} \mathbf{v}_{h}, \operatorname{grad} \psi_{h}\right)_{\boldsymbol{L}^{2}(\Omega)}}{\left\|\mathbf{v}_{h}\right\|_{\boldsymbol{H}(\mathbf{c u r l} ; \Omega)}} & \geq \frac{\left(\boldsymbol{\epsilon} \operatorname{grad} \psi_{h}, \operatorname{grad} \psi_{h}\right)_{\boldsymbol{L}^{2}(\Omega)}}{\left|\psi_{h}\right|_{H^{1}(\Omega)}} \\
& \geq C\left|\psi_{h}\right|_{H^{1}(\Omega)} \geq C_{0}\left\|\psi_{h}\right\|_{H^{1}(\Omega)}
\end{aligned}
$$

for $\psi_{h} \in \mathcal{W}_{p, 0}^{0}\left(\Omega_{h}\right)$. Standard estimates finish the proof.

The conditions on the bilinear form $d(\cdot, \cdot)$ are very weak. For instance, a lumped $\boldsymbol{L}^{2}(\Omega)$-inner product according to (3.37) will meet the requirements and lead to a diagonal matrix D. Hence, the matrix $\mathrm{A}_{\boldsymbol{\mu}}+s \mathrm{GD}^{-1} \mathrm{G}^{T}$ in (6.1) remains sparse and can be assembled efficiently. This procedure is called 'discrete regularization', because the manipulations are carried out entirely on the matrix level.

Beside the physically meaningful discrete eigenvalues/eigenfunctions, we get many more from (6.1). The additional non-physical solutions can easily be weeded out by looking at $\varphi_{h}=\mathrm{D}^{-1} \mathrm{G}^{T} \overrightarrow{\mathbf{e}}_{h}$. If this is nonzero, the eigenfunction can be dismissed.

Discrete regularization can also be performed for the source problem, for instance in the case of the stabilized problem of Section 5.4. Further, assuming trivial topology, some preprocessing is required, by replacing $\mathbf{j} \leftarrow \mathbf{j}-\operatorname{grad} \eta_{h}$, where $\eta_{h} \in \mathcal{W}_{p}^{0}\left(\Omega_{h}\right)$ satisfies

$$
\left(\operatorname{grad} \eta_{h}, \operatorname{grad} \eta_{h}^{\prime}\right)_{\boldsymbol{L}^{2}(\Omega)}=\left(\mathbf{j}, \operatorname{grad} \eta_{h}^{\prime}\right)_{\boldsymbol{L}^{2}(\Omega)} \quad \forall \eta_{h}^{\prime} \in \mathcal{W}_{p}^{0}\left(\Omega_{h}\right)
$$

Using this modified weakly discretely divergence-free $\mathbf{j}$, the solution for $v_{h}$ turns out to be zero and the same technique as above can be applied. Then $\mathbf{a}_{h}$ can be determined by solving a system with the Hermitian matrix

$$
\mathrm{A}_{\boldsymbol{\mu}}+s \mathrm{GD}^{-1} \mathrm{G}^{T}-\omega^{2} \mathrm{M}_{\boldsymbol{\epsilon}}
$$


This matrix has a small fixed number of negative eigenvalues, just like the matrix arising from the Helmholtz boundary value problem.

\section{Bibliographical notes}

In Bespalov (1988) the discrete regularization idea was introduced for the treatment of electromagnetic eigenproblems. A discussion is given in Adam et al. (1997, Section 6). This latter report gives a rather comprehensive account of the numerical treatment of regularized Maxwell eigenvalue problems. Discrete regularization is a fairly natural idea from the perspective of discrete Hodge operators ( $c f$. Section 3.3) and is frequently treated in articles on the finite integration technique (Clemens and Weiland 2001, Section 3). Furthermore, an approach resembling discrete regularization is the gist of the paper by Haber, Ascher, Aruliah and Oldenburg (1999).

\section{2. grad-div regularization}

The matrix $\mathrm{GD}^{-1} \mathrm{G}^{T}$ used above induces an inner product for weakly defined discrete divergences. An analogous regularization can also be carried out on the continuous level. It is motivated by observing that, in the eigenvalue problem (4.1), we necessarily have $\mathbf{e} \in \boldsymbol{Z}_{0}(\boldsymbol{\epsilon}, \Omega)$, which implies $\operatorname{div}(\boldsymbol{\epsilon} \mathbf{e})=0$. We are led to consider the following related variational eigenvalue problem. For $s>0$ find $\mathbf{e} \in \boldsymbol{X}_{0}(\boldsymbol{\epsilon}, \Omega), \omega \neq 0$ with

$$
\left(\boldsymbol{\mu}^{-1} \operatorname{curl} \mathbf{e}, \operatorname{curl} \mathbf{e}^{\prime}\right)_{\boldsymbol{L}^{2}(\Omega)}+s\left(\operatorname{div}(\boldsymbol{\epsilon} \mathbf{e}), \operatorname{div}\left(\boldsymbol{\epsilon} \mathbf{e}^{\prime}\right)\right)_{L^{2}(\Omega)}=\omega^{2}\left(\boldsymbol{\epsilon} \mathbf{e}, \mathbf{e}^{\prime}\right)_{\boldsymbol{L}^{2}(\Omega)}
$$

for all $\mathbf{e}^{\prime} \in \boldsymbol{X}_{0}(\boldsymbol{\epsilon}, \Omega)$. By Corollary 4.3 this is an eigenvalue problem for an operator with a compact resolvent in $\boldsymbol{L}^{2}(\Omega)$. The true Maxwell eigenvalues will not depend on the regularization parameter $s$, and the uninteresting solutions can be distinguished by nonzero $\operatorname{div}(\boldsymbol{\epsilon} \cdot)$. The strong form of (6.2) seems to be

$$
\operatorname{curl} \boldsymbol{\mu}^{-1} \operatorname{curl} \mathbf{e}-s \epsilon \operatorname{grad} \operatorname{div}(\epsilon \mathbf{e})=\omega^{2} \boldsymbol{\epsilon} .
$$

This accounts for the parlance 'grad-div regularization'. For the Maxwell source problem a similar strategy can be pursued. For simplicity assume $\operatorname{div} \mathbf{j}=0$ and trivial topology. Then the grad-div regularized formulation of (5.1) is as follows. Seek $\mathbf{e} \in \boldsymbol{X}_{0}(\boldsymbol{\epsilon}, \Omega)$ such that

$$
\begin{array}{r}
\left(\boldsymbol{\mu}^{-1} \operatorname{curl} \mathbf{e}, \operatorname{curl} \mathbf{e}^{\prime}\right)_{\boldsymbol{L}^{2}(\Omega)}+s\left(\operatorname{div}(\boldsymbol{\epsilon} \mathbf{e}), \operatorname{div}\left(\boldsymbol{\epsilon} \mathbf{e}^{\prime}\right)\right)_{\boldsymbol{L}^{2}(\Omega)}-\omega^{2}\left(\boldsymbol{\epsilon e}, \mathbf{e}^{\prime}\right)_{\boldsymbol{L}^{2}(\Omega)} \\
=-i \omega\left(\mathbf{j}, \mathbf{e}^{\prime}\right)_{\boldsymbol{L}^{2}(\Omega)}
\end{array}
$$

for all $\mathbf{e}^{\prime} \in \boldsymbol{X}_{0}(\boldsymbol{\epsilon}, \Omega)$. Equivalence of (6.4) and (5.1) is not straightforward, and tied to conditions ( $c f$. Costabel and Dauge (1998, Theorem 1.1)).

Lemma 6.2. If $\omega^{2} / s$ is not an eigenvalue of the operator $\operatorname{div}(\boldsymbol{\epsilon} \operatorname{grad} \cdot)$ on $H_{0}^{1}(\Omega)$, then (6.4) and (5.1) have the same solutions. 
Proof. It is clear that solutions of (5.1) also solve (6.4). To prove the converse, we only have to show that $\operatorname{div}(\boldsymbol{\epsilon} \mathbf{e})=0$ for any solution of (6.4). Testing with gradients of functions in $H_{0}^{1}(\Omega)$ shows

$$
\left(\operatorname{div}(\boldsymbol{\epsilon} \mathbf{e}), s \operatorname{div}(\boldsymbol{\epsilon} \operatorname{grad} \psi)+\omega^{2} \psi\right)_{\boldsymbol{L}^{2}(\Omega)}=0 \quad \forall \psi \in H_{0}^{1}(\Omega) .
$$

We have $\left(s \operatorname{div}(\epsilon \operatorname{grad} \cdot)+\omega^{2} \mathrm{Id}\right) H_{0}^{1}(\Omega)=L^{2}(\Omega)$, provided that $\omega, s$ satisfy the assumptions of the theorem.

To keep the presentation simple, we set $\boldsymbol{\epsilon}=\mathbf{I}$ for the remainder of this section. We may ask ourselves how (6.2) and (6.4) should be discretized by means of finite elements. Any scheme must be both curl- and divconforming, meaning both tangential and normal continuity. It goes without saying that this amounts to global continuity for any piecewise smooth vector-field. Thus we have to leave the framework of discrete differential forms and approximate $\mathbf{e}$ in the space $\mathcal{S}_{p, 0}\left(\Omega_{h}\right):=\left(\mathcal{W}_{p}^{0}\left(\Omega_{h}\right)\right)^{3} \cap \boldsymbol{H}_{0}(\mathbf{c u r l} ; \Omega)$ of vector-fields with continuous, piecewise polynomial Cartesian components. Seek $\widetilde{\mathbf{e}} \in \mathcal{S}_{p, 0}\left(\Omega_{h}\right)$ such that

$$
\begin{gathered}
\left(\boldsymbol{\mu}^{-1} \operatorname{curl} \widetilde{\mathbf{e}}_{h}, \operatorname{curl} \widetilde{\mathbf{e}}_{h}^{\prime}\right)_{\boldsymbol{L}^{2}(\Omega)}+s\left(\operatorname{div}(\mathbf{e}), \operatorname{div}\left(\widetilde{\mathbf{e}}_{h}^{\prime}\right)\right)_{\boldsymbol{L}^{2}(\Omega)}-\omega^{2}\left(\widetilde{\mathbf{e}}_{h}, \widetilde{\mathbf{e}}_{h}^{\prime}\right)_{\boldsymbol{L}^{2}(\Omega)} \\
=-i \omega\left(\mathbf{j}, \widetilde{\mathbf{e}}_{h}^{\prime}\right)_{\boldsymbol{L}^{2}(\Omega)}
\end{gathered}
$$

for all $\widetilde{\mathbf{e}}_{h}^{\prime} \in \mathcal{S}_{p, 0}\left(\Omega_{h}\right)$.

The desire to get continuous approximations of e actually provides a motivation for using the grad-div regularization for the source problem in the case $\operatorname{div} \mathbf{j}=0$. We saw in Section 4.5 that the space $\mathcal{S}_{p, 0}\left(\Omega_{h}\right)$ must be ruled out for the direct discretization of (5.1), because on general meshes the spectrum of the discrete system matrix is polluted by kernel components. This might lead to severely ill-conditioned linear systems, even if $\omega$ is far away from any Maxwell eigenvalue. The grad-div regularized formulations can be used to suppress this disastrous pollution effect and thus apparently paves the way for applying Lagrangian finite elements by means of $\mathcal{S}_{p, 0}\left(\Omega_{h}\right)$. One can cite several 'practical' reasons why one might prefer $\mathbf{e}_{h} \in \mathcal{S}_{p, 0}\left(\Omega_{h}\right)$ to the approximation by a discrete 1-form.

- The electromagnetic problem should be solved with an existing FEM software package that can only handle vertex-based degrees of freedom.

- One might 'trust' traditional finite elements more than discrete differential forms.

- The result is needed as input data for another computation which might be crippled by discontinuities. Allegedly, this is the case for particle simulations (Maxwell-Vlasov equations). 
Is (6.4) actually the continuous problem underlying (6.5)? Evidently, $\mathcal{S}_{p, 0}\left(\Omega_{h}\right) \subset \boldsymbol{H}^{1}(\Omega)$, and so (6.5) can be regarded as a Galerkin discretization of $(6.4)$ posed over the space

$$
\boldsymbol{H}_{\times}^{1}(\Omega):=\boldsymbol{H}^{1}(\Omega) \cap \boldsymbol{H}_{0}(\operatorname{curl} ; \Omega) .
$$

This is not a moot point, because any $\widetilde{\mathbf{u}} \in \boldsymbol{H}_{\times}^{1}(\Omega)$ can be arbitrarily well approximated by functions in $\mathcal{S}_{p, 0}\left(\Omega_{h}\right)$, provided the meshwidth is small enough or the polynomial degree $p$ is large enough (Bonnet-BenDhia et al. 1999, Appendix). If the same is true for $\boldsymbol{X}_{0}(\mathbf{I}, \Omega)$, then (6.5) really discretizes (6.4). Be aware that this kind of 'density assumption' is inherent in the notion of Galerkin approximation.

A first observation stirs suspicion. Testing (6.4) with $\mathbf{e}^{\prime} \in \operatorname{grad}\left(H^{2}(\Omega) \cap\right.$ $\left.H_{0}^{1}(\Omega)\right) \subset \boldsymbol{H}_{\times}^{1}(\Omega)$, we find

$$
\left(\operatorname{div}(\mathbf{e}), s \Delta \psi+\omega^{2} \psi\right)_{L^{2}(\Omega)}=0 \quad \forall \psi \in H^{2}(\Omega) \cap H_{0}^{1}(\Omega) .
$$

However, it is well known that $\left(s \Delta+\omega^{2} \operatorname{Id}\right)\left(H^{2}(\Omega) \cap H_{0}^{1}(\Omega)\right)$ is a closed subspace of $L^{2}(\Omega)$ (Hanna and Smith 1967, Dauge 1988), and a proper subspace in the presence of non-convex edges of $\Omega$; then the Dirichlet problem for $s \Delta+\omega^{2}$ Id with right-hand side $\in L^{2}(\Omega)$ may have solutions that are not in $H^{2}(\Omega)$. We conclude that $\operatorname{div} \mathbf{e}=0$ is not guaranteed, if (6.4) is considered on $\boldsymbol{H}_{\times}^{1}(\Omega)$. The situation is strikingly disclosed by the following result.

Theorem 6.3. The space $\boldsymbol{H}_{\times}^{1}(\Omega)$ is a closed subspace of $\boldsymbol{X}_{0}(\mathbf{I}, \Omega)$, and the inclusion is strict, if $\Omega$ has re-entrant edges or corners.

Proof. Let $\left(\widetilde{\mathbf{u}}_{n}\right)_{n \in \mathbb{N}}$ be a Cauchy sequence in $\boldsymbol{X}_{0}(\mathbf{I}, \Omega)$ whose members belong to $\boldsymbol{H}_{\times}^{1}(\Omega)$. By Lemma 2.4 we find Cauchy sequences $\left(\mathbf{v}_{n}\right)_{n \in \mathbb{N}}, \mathbf{v}_{n}:=$ $\mathrm{R}_{0} \widetilde{\mathbf{u}}_{n}$, in $\boldsymbol{H}_{\times}^{1}(\Omega)$, and $\left(\varphi_{n}\right)_{n}, \varphi_{n}:=\mathrm{N}_{0} \widetilde{\mathbf{u}}_{n}$, in $H(\Delta, \Omega)$, where

$$
H(\Delta, \Omega):=\left\{\phi \in H_{0}^{1}(\Omega), \Delta \phi \in L^{2}(\Omega)\right\},
$$

with

$$
\widetilde{\mathbf{u}}_{n}=\mathbf{v}_{n}+\operatorname{grad} \varphi_{n} \quad \forall n \in \mathbb{N} .
$$

As $\left(\varphi_{n}\right)_{n}$ is also a sequence in $H^{2}(\Omega) \cap H_{0}^{1}(\Omega)$ and this space is closed in $H(\Delta, \Omega)$ (Hanna and Smith 1967 ), the first assertion follows. The second is immediate from considering gradients of functions in $H(\Delta, \Omega) \backslash\left(H^{2}(\Omega) \cap\right.$ $H_{0}^{1}(\Omega)$ ). See Amrouche et al. (1998, Section 2.c) for a concrete example.

This theorem sends the important message that, if $\Omega$ has re-entrant edges, we may get different solutions $\mathbf{e}$ and $\widetilde{\mathbf{e}}$ when considering the variational equation (6.4) on $\boldsymbol{X}_{0}(\mathbf{I}, \Omega)$ and $\boldsymbol{H}_{\times}^{1}(\Omega)$, respectively. More precisely, $\widetilde{\mathbf{e}}$ is the Galerkin projection of e onto $\boldsymbol{H}_{\times}^{1}(\Omega)$. As regards the finite element approximation in $\mathcal{S}_{p, 0}\left(\Omega_{h}\right)$, Theorem 6.3 contends that in $\mathcal{S}_{p, 0}\left(\Omega_{h}\right)$ we may not be able to get arbitrarily close to e in the $\boldsymbol{X}_{0}(\mathbf{I}, \Omega)$-norm, which is 


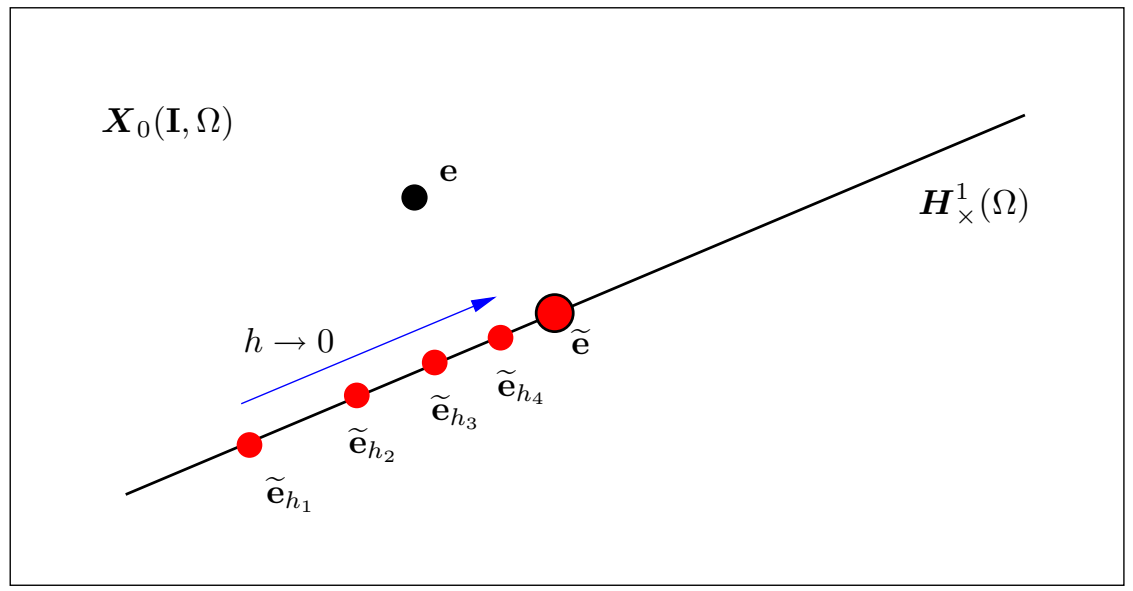

Figure 6.1. 'Convergence' of finite element approximations to the solution of (6.4). The geometry of the plane of the paper is induced by the $\boldsymbol{X}_{0}(\mathbf{I}, \Omega)$-metric

the right energy norm. This behaviour is illustrated in Figure 6.1. The eigenvalue problem (6.2) is afflicted as well. In other words, even if $\mathbb{H}$ is a sequence of meshwidths tending to zero, $\bigcup_{h \in \mathbb{H}} \mathcal{S}_{p, 0}\left(\Omega_{h}\right)$ is not dense in $\boldsymbol{X}_{0}(\mathbf{I}, \Omega)$, if there are re-entrant edges/corners.

This phenomenon is perilous, because the discrete solutions will 'look' correct and even display nice convergence as the meshes are refined. The unwary will be lulled into a false sense of security and happily accept a possibly wrong result.

Remark 19. Most readers will know the identity

$$
-\Delta=\text { curl curl - grad div, }
$$

which holds for $C^{2}$-vector-fields and, therefore, in the sense of distributions. Crudely speaking, the grad-div regularization aims to exploit this identity and switch to a variational problem in $\boldsymbol{H}_{\times}^{1}(\Omega)$. This is prone to failure, because Theorem 6.3 tells us that smooth vector-fields with vanishing tangential components on $\partial \Omega$ are not necessarily dense in $\boldsymbol{X}_{0}(\mathbf{I}, \Omega)$. As a consequence, the variational problem $(6.4)$ on $\boldsymbol{X}_{0}(\mathbf{I}, \Omega)$ cannot be stated as an equivalent partial differential equation in the sense of distributions. Now we understand that the partial differential equation (6.3) actually belongs to the problem on $\boldsymbol{H}_{\times}^{1}(\Omega)$ ( $c f$. Bonnet-BenDhia et al. (1999, Section 4)).

Remark 20. As shown by the investigations in Costabel and Dauge (1998) and Costabel et al. (1999), the gap between $\boldsymbol{X}_{0}(\mathbf{I}, \Omega)$ and $\boldsymbol{H}_{\times}^{1}(\Omega)$ can be filled with gradients of singular functions associated with re-entrant edges of 
the domain boundary. Thus, the singular field method aims to augment the space $\mathcal{S}_{p, 0}\left(\Omega_{h}\right)$ by these singular functions (Bonnet-BenDhia et al. 1999). Unfortunately the space spanned by the singular functions has infinite dimension, which thwarts a straightforward application of the idea. On the contrary, it is successful for reduced two-dimensional problems (Hazard and Lohrengel 2000, Assous, Ciarlet Jr and Sonnendrücker 1998).

Remark 21. Also, the presence of discontinuities in $\boldsymbol{\mu}$ and $\boldsymbol{\epsilon}$ might rule out the discretization of (6.2), even if finite element spaces are used that belong to $\boldsymbol{H}^{1}\left(\Omega_{i}\right)$ only for subdomains $\Omega_{i}$ of $\Omega$, where the coefficients are smooth: non-density will strike again. We refer to the discussion in Section 6.2 of Bonnet-BenDhia et al. (1999), Costabel et al. (1999, Section 2) and Lohrengel and Nicaise (2001).

\section{Bibliographical notes}

Grad-div regularization is an established technique in electromagnetic scattering theory Hazard and Lenoir (1996), Werner (1963) and Leis (1986, Section 8.4). The non-density result of Theorem 6.3 was discovered by M. Costabel (1991). A thorough analysis along with numerical experiments is given in Costabel and Dauge (1999). In this paper it is pointed out that considering (6.2) on $\boldsymbol{H}_{\times}^{1}(\Omega)$ amounts to switching from electromagnetism to a setting for linear elasticity. The case of discontinuous coefficients is treated in Lohrengel and Nicaise (2001).

\subsection{Weighted regularization}

When carrying out grad-div regularization we squandered a lot of freedom by opting for the $\boldsymbol{L}^{2}(\Omega)$-inner product of the divergence terms right from the beginning. We could have considered a generic regularized problem of the following form. Find $\mathbf{e} \in \boldsymbol{X}_{0}[Y](\Omega)$ such that

$$
\begin{aligned}
\left(\boldsymbol{\mu}^{-1} \operatorname{curl} \mathbf{e}, \operatorname{curl} \mathbf{e}^{\prime}\right)_{\boldsymbol{L}^{2}(\Omega)}+\left\langle\operatorname{div} \mathbf{e}, \operatorname{div} \mathbf{e}^{\prime}\right\rangle_{Y}-\omega^{2} & \left(\mathbf{e}, \mathbf{e}^{\prime}\right)_{\boldsymbol{L}^{2}(\Omega)} \\
& =-i \omega\left(\mathbf{j}, \mathbf{e}^{\prime}\right)_{\boldsymbol{L}^{2}(\Omega)}
\end{aligned}
$$

for all $\mathbf{e}^{\prime} \in \boldsymbol{X}_{0}[Y](\Omega)$, where $Y$ is some Hilbert space with inner product $\langle\cdot, \cdot\rangle_{Y}$ and

$$
\boldsymbol{X}_{0}[Y](\Omega):=\left\{\mathbf{u} \in \boldsymbol{H}_{0}(\mathbf{c u r l} ; \Omega), \operatorname{div} \mathbf{u} \in Y\right\} .
$$

In the previous section we saw that $Y=\boldsymbol{L}^{2}(\Omega)$ fails to ensure density of $\boldsymbol{H}_{\times}^{1}(\Omega)$ in $\boldsymbol{X}_{0}[Y](\Omega)$. On the other hand, $Y=\boldsymbol{H}^{-1}(\Omega)$ recovers $\boldsymbol{X}_{0}[Y](\Omega)=$ $\boldsymbol{H}_{0}(\boldsymbol{c u r l} ; \Omega)$, which gives the correct solution, namely that of the nonregularized problem. However, it fails to be compactly embedded into $\boldsymbol{L}^{2}(\Omega)$ and, therefore, is haunted by kernel pollution when discretized by means of 
$\mathcal{S}_{p, 0}\left(\Omega_{h}\right)$. Is there a middle ground, that is, a Hilbert space $Y$,

$$
\boldsymbol{L}^{2}(\Omega) \subset Y \subset \boldsymbol{H}^{-1}(\Omega),
$$

that meets both

- $\boldsymbol{H}_{\times}^{1}(\Omega)$ is dense in $\boldsymbol{X}_{0}[Y](\Omega)$, and

- $\boldsymbol{X}_{0}[Y](\Omega)$ is compactly embedded into $\boldsymbol{L}^{2}(\Omega)$ ?

To state sufficient and necessary conditions, when this is the case, we rely on the Hilbert space

$$
H(\Delta[Y], \Omega):=\left\{\varphi \in H_{0}^{1}(\Omega), \Delta \varphi \in Y\right\},
$$

and the associated Riesz operator

$$
\text { I : } H(\Delta[Y], \Omega) \mapsto Y, \quad\langle\psi, \mid \varphi\rangle_{Y}:=\langle\varphi, \psi\rangle_{H^{1}(\Omega) \times H^{-1}(\Omega)} \quad \forall \psi \in Y .
$$

As in the case of Lemma 6.2 one proves the equivalence of (6.6) and (5.1) (cf. Costabel and Dauge (2002, Theorem 2.1)).

Lemma 6.4. If $\operatorname{div} \mathbf{j}=0$ and $\left(s \Delta+\omega^{2} \mathrm{I}\right) H(\Delta[Y], \Omega)$ is dense in $Y$, then the solutions of (6.6) and (5.1) coincide.

The main technical tool for further investigations is another regular splitting theorem (Costabel and Dauge 2002, Theorem 2.2).

Lemma 6.5. (Regular splitting for $\boldsymbol{X}_{0}[Y](\Omega)$ ) If $Y$ satisfies (6.7) we find continuous mappings $\mathrm{R}_{0}[Y]: Y \mapsto \boldsymbol{H}_{\times}^{1}(\Omega), \mathrm{N}_{0}[Y]: Y \mapsto H(\Delta[Y], \Omega)$ such that $\mathrm{R}_{0}[Y]+\operatorname{grad} \circ \mathrm{N}_{0}[Y]=\mathrm{Id}$.

Proof. We can re-use the operators $\mathrm{R}_{0}$ and $\mathrm{N}_{0}$ from Lemma 2.4 after noting that

$$
\left\|\Delta\left(\mathrm{N}_{0} \mathbf{u}\right)\right\|_{Y} \leq C\left(\|\operatorname{div} \mathbf{u}\|_{Y}+\left\|\operatorname{div}\left(\mathrm{R}_{0} \mathbf{u}\right)\right\|_{L^{2}(\Omega)}\right) .
$$

Thus $\mathrm{R}_{0}[Y]$ and $\mathrm{N}_{0}[Y]$ can be chosen as plain restrictions of $\mathrm{R}_{0}$ and $\mathrm{N}_{0}$ to $Y$.

As remarked in Costabel and Dauge (2002, Section 2.2) this allows two important conclusions.

Corollary 6.6. If the embedding $Y \hookrightarrow \boldsymbol{H}^{-1}(\Omega)$ is compact, the same is true for $\boldsymbol{X}_{0}[Y](\Omega) \hookrightarrow \boldsymbol{L}^{2}(\Omega)$.

Corollary 6.7. If $H^{2}(\Omega) \cap H_{0}^{1}(\Omega)$ is a dense subspace of $H(\Delta[Y], \Omega)$, then $\boldsymbol{H}_{\times}^{1}(\Omega)$ is dense in $\boldsymbol{X}_{0}[Y](\Omega)$.

Essentially, issues concerning the choice of $Y$ can be investigated by looking at the Laplacian on polyhedral domains. Its theory is a venerable branch of the theory of elliptic boundary value problems. A key insight gained for second-order elliptic operators on polyhedra (Nazarov and 
Plamenevskii 1994, Kondrat'ev 1967) is that it is weighted Sobolev spaces that supply the appropriate functional framework. This is why weighted $L^{2}$ spaces are the most promising candidates for $Y$, suggesting that we define

$$
\|u\|_{Y}:=\int_{\Omega} w(\mathbf{x})^{2}|u|^{2} \mathrm{~d} \mathbf{x},
$$

with a positive weight function $\left(\gamma_{\mathbf{v}}, \gamma_{e} \in \mathbb{R}\right)$

$$
w(\mathbf{x}):=\prod_{\text {corners } \mathbf{v}} \operatorname{dist}(\mathbf{x} ; \mathbf{v})^{\gamma_{\mathbf{v}}} \cdot \prod_{\text {edges } e} \widetilde{\operatorname{dist}}(\mathbf{x} ; e)^{\gamma_{e}},
$$

where, with endpoints $\mathbf{v}_{1}, \mathbf{v}_{2}$ of $e$,

$$
\widetilde{\operatorname{dist}}(\mathbf{x} ; e):=\frac{\operatorname{dist}(\mathbf{x} ; e)}{\operatorname{dist}\left(\mathbf{v}_{1} ; e\right) \operatorname{dist}\left(\mathbf{v}_{2} ; e\right)} \text {. }
$$

Here $\mathbf{v}, e$ run through the corners and edges of the boundary of $\Omega$, respectively. Profound results about the singularities of the Laplacian supply a precise recipe for how to choose the weight exponents $\gamma_{\mathbf{v}}$ and $\gamma_{e}$ (Costabel and Dauge 2002, Section 4).

Theorem 6.8. (Choice of weight exponents) If $\frac{1}{2} \leq \gamma_{\mathbf{v}}<1,0 \leq \gamma_{e}<1$ and

$$
\gamma_{e}>1-\min _{\mathbf{x} \in e} \frac{\pi}{\omega_{e}(\mathbf{x})}
$$

where $\omega_{e}(\mathbf{x})$ is the opening angle for the (curved) edge $e$ at point $\mathbf{x} \in e$, then $\boldsymbol{X}_{0}[Y](\Omega) \hookrightarrow \boldsymbol{L}^{2}(\Omega)$ compactly and $\boldsymbol{H}_{\times}^{1}(\Omega) \subset \boldsymbol{X}_{0}[Y](\Omega)$ is dense.

Choosing the weight exponents according to the assumptions of the theorem and using a Galerkin discretization of (6.6) based on $\mathcal{S}_{p, 0}\left(\Omega_{h}\right)$ gives a scheme for the source problems that is asymptotically optimal on sufficiently fine meshes. This is immediately clear from applying the theory of Galerkin approximations for coercive variational problems. The compact embedding $\boldsymbol{X}_{0}[Y](\Omega) \hookrightarrow \boldsymbol{L}^{2}(\Omega)$ makes (6.6) belong to this class. For the same reason the standard theory can also be applied to the regularized eigenvalue problem. Asymptotic optimality can then be concluded with respect to the computed eigenvalues and eigenfunctions.

Remark 22. The idea of weighted regularization is too new to assess its merits. As for the discrete regularization of Section 6.1, it will produce 'nice' linear systems. In addition, we are rewarded with a globally continuous solution for the electric field. On the other hand, when compared with discrete 1-forms, a few drawbacks are obvious. 
- Even for constant material parameters, the assembly of the linear system is somewhat complicated and entails using appropriate numerical quadrature.

- Given only raw geometry data in the form of a mesh, it might be costly to compute the weight functions.

- For non-constant coefficient $\boldsymbol{\epsilon}$ the evaluation of $\operatorname{div}\left(\boldsymbol{\epsilon} \mathbf{e}_{h}\right)$ requires a suitable approximation.

- If $\boldsymbol{\epsilon}$ has discontinuities, demanding that the normal component of $\boldsymbol{\epsilon} \mathbf{e}_{h}$ is continuous necessitates using a discontinuous trial space instead of $\mathcal{S}_{p, 0}\left(\Omega_{h}\right)$. This might rule out the use of standard FEM software, which is a main point in favour of regularized formulations.

\section{Bibliographical notes}

Weighted regularization has been explored in the ground-breaking work by M. Costabel and M. Dauge (2002), which we followed closely in our presentation. There the reader can find all the details, and also refined a priori estimates for the discretization errors encountered for the weighted regularized formulation and certain finite element spaces.

\section{Conclusion and further issues}

This article has aimed to elucidate a few fundamental techniques for the analysis of finite element schemes for electromagnetic problems in the frequency domain. The focus was both on the design of suitable 'physical' finite elements and on the functional analytic techniques necessary to establish results on asymptotic convergence on shape-regular meshes. The discussion has been restricted to a few simple model problems, which, however, display all the subtle difficulties encountered by finite element Galerkin schemes in computational electromagnetism.

Theoretical computational electromagnetism is a huge area and this article can cover only a fraction of it. Key topics that have not been addressed are:

- Numerical methods in the time domain (Taflove 1995, Clemens and Weiland 1999).

- Boundary element methods for scattering problems and eddy current computation and their coupling with finite elements (Nédélec 2001, Colton and Kress 1998, Buffa et al. 2002, Hiptmair 2000, Christiansen 2000, Bendali 1984a, Bendali 1984b, Kuhn and Steinbach 2001).

- Absorbing boundary conditions (Grote and Keller 1998, Teixeira and Chew 1998, Sacks, Kingsland, Lee and Lee 1995). 
- Fast iterative solvers and domain decomposition techniques for eddy current and scattering problems (Hiptmair 1999b, Hiptmair and Toselli 1999, Gopalakrishnan and Pasciak 2000, Buffa, Mayday and Rapetti 2001, Hoppe 1999).

- the design and analysis of a posteriori error estimators (Beck, Hiptmair, Hoppe and Wohlmuth 2000b, Beck, Hiptmair and Wohlmuth 2000a, Beck, Deuflhard, Hiptmair, Hoppe and Wohlmuth 1998, Monk 1999).

\section{Acknowledgement}

I am very grateful to R. Kotiuga, M. Dauge, M. Costabel, D. Boffi, Z. Chen, V. Gradinaru and S. Kurz, who suggested numerous improvements to the first draft of this survey article.

\section{REFERENCES}

S. Adam, P. Arbenz and R. Geus (1997), Eigenvalue solvers for electromagnetic fields in cavities. Technical Report 275, Institute of Scientific Computing, ETH Zürich, Zürich, Switzerland.

R. Adams (1975), Sobolev Spaces, Academic Press, New York.

M. Ainsworth and J. Coyle (2001a), Conditioning of hierarchic $p$-version Nédélec elements on meshes of curvilinear quadrilaterals and hexahedra. Report 26, Department of Mathematics, University of Strathclyde, Glasgow, UK.

M. Ainsworth and J. Coyle (2001b), 'Hierarchic $h p$-edge element families for Maxwell's equations on hybrid quadrilateral/triangular meshes', Comput. Meth. Appl. Mech. Engr. 190, 6709-6733.

M. Ainsworth and K. Pinchedez (2001), $h p$-approximation theory for BDM/RT finite elements and applications. Report 15, Department of Mathematics, University of Strathclyde, Glasgow, UK.

A. Alonso and A. Valli (1999), 'An optimal domain decomposition preconditioner for low-frequency time-harmonic Maxwell equations', Math. Comp. 68, 607631.

C. Amrouche, C. Bernardi, M. Dauge and V. Girault (1998), 'Vector potentials in three-dimensional nonsmooth domains', Math. Meth. Appl. Sci. 21, 823-864.

P. Anselone (1971), Collectively Compact Operator Approximation Theory and Applications to Integral Equations, Prentice-Hall, Englewood Cliffs, NJ.

T. Apel (1999), Anisotropic Finite Elements: Local Estimates and Applications, Advances in Numerical Mathematics, Teubner, Stuttgart, Germany.

T. Apel and M. Dobrowolski (1992), 'Anisotropic interpolation with applications to the finite element method', Computing 47, 277-293.

T. Apel, S. Nicaise and J. Schöberl (2001), 'Crouzeix-Raviart type finite elements on anisotropic meshes', Numer. Math. 89, 193-223.

F. Assous, P. Ciarlet Jr and E. Sonnendrücker (1998), 'Resolution of Maxwell equations in a domain with reentrant corners', RAIRO M2AN 32, 359-389. 
I. Babuška (1971), 'Error bounds for the finite element method', Numer. Math. 16, 322-333.

I. Babuška and J. Osborn (1991), Eigenvalue problems, in Finite Element Methods, Part 1 (P. G. Ciarlet and J. Lions, eds), Vol. 2 of Handbook of Numerical Analysis, Elsevier, Amsterdam, pp. 641-787.

I. Babuška and M. Suri (1994), 'The $p$ and $h p$ versions of the finite element method: Basic principles and properties', SIAM Review 36, 578-632.

I. Babuška, A. Craig, J. Mandel and J. Pitkäranta (1991), 'Efficient preconditioning for the $p$-version finite element method in two dimensions', SIAM J. Numer. Anal. 28, 624-661.

D. Baldomir (1986), 'Differential forms and electromagnetism in 3-dimensional Euclidean space $\mathbb{R}^{3}$, IEE Proc. A 133, 139-143.

D. Baldomir and P. Hammond (1996), Geometry of Electromagnetic Systems, Clarendon Press, Oxford.

R. Beck, P. Deuflhard, R. Hiptmair, R. Hoppe and B. Wohlmuth (1998), 'Adaptive multilevel methods for edge element discretizations of Maxwell's equations', Surveys on Mathematics for Industry 8, 271-312.

R. Beck, R. Hiptmair and B. Wohlmuth (2000a), A hierarchical error estimator for eddy current computation, in ENUMATH 99: Proceedings of the 3rd European Conference on Numerical Mathematics and Advanced Applications, Jyväskylä, Finland, July 26-30 (P. Neittaanmäki and T. Tiihonen, eds), World Scientific, Singapore, pp. 110-120.

R. Beck, R. Hiptmair, R. Hoppe and B. Wohlmuth (2000b), 'Residual based $a-$ posteriori error estimators for eddy current computation', RAIRO M2AN 34, 159-182.

A. Bendali (1984a), 'Numerical analysis of the exterior boundary value problem for time harmonic Maxwell equations by a boundary finite element method, Part 1: The continuous problem', Math. Comp. 43, 29-46.

A. Bendali (1984b), 'Numerical analysis of the exterior boundary value problem for time harmonic Maxwell equations by a boundary finite element method, Part 2: The discrete problem', Math. Comp. 43, 47-68.

A. Bespalov (1988), 'Finite element method for the eigenmode problem of a RF cavity resonator', Soviet J. Numer. Anal. Math. Model. 3, 163-178.

M. Birman and M. Solomyak (1990), 'Construction in a piecewise smooth domain of a function of the class $H^{2}$ from the value of the conormal derivative', J. Math. Sov. 49, 1128-1136.

D. Boffi (2000), 'Discrete compactness and Fortin operator for edge elements', Numer. Math. 87, 229-246.

D. Boffi and L. Gastaldi (2001), 'Edge finite elements for the approximation of Maxwell resolvent operator'. Submitted to RAIRO M2AN.

D. Boffi, F. Brezzi and L. Gastaldi (2000), 'On the problem of spurious eigenvalues in the approximation of linear elliptic problems in mixed form', Math. Comp. 69, 121-140.

D. Boffi, P. Fernandes, L. Gastaldi and I. Perugia (1999), 'Computational models of electromagnetic resonators: Analysis of edge element approximation', SIAM J. Numer. Anal. 36, 1264-1290. 
A. Bonnet-BenDhia, C. Hazard and S. Lohrengel (1999), 'A singular field method for the solution of Maxwell's equations in polyhedral domains', SIAM J. Appl. Math. 59, 2028-2044.

A. Bossavit (1988a), Mixed finite elements and the complex of Whitney forms, in The Mathematics of Finite Elements and Applications VI (J. Whiteman, ed.), Academic Press, London, pp. 137-144.

A. Bossavit (1988b), 'A rationale for edge elements in 3D field computations', IEEE Trans. Magnetics 24, 74-79.

A. Bossavit (1988c), 'Whitney forms: A class of finite elements for threedimensional computations in electromagnetism', IEE Proc. A 135, 493-500.

A. Bossavit (1992), 'A new viewpoint on mixed elements', Meccanica 27, 3-11.

A. Bossavit (1998a), Computational Electromagnetism. Variational Formulation, Complementarity, Edge Elements, Vol. 2 of Electromagnetism Series, Academic Press, San Diego, CA.

A. Bossavit (1998b), 'On the geometry of electromagnetism I: Affine space', J. Japan Soc. Appl. Electromagnetics Mech. 6, 17-28.

A. Bossavit (1998c), 'On the geometry of electromagnetism II: Geometrical objects', J. Japan Soc. Appl. Electromagnetics Mech. 6, 114-123.

A. Bossavit (1998d), 'On the geometry of electromagnetism III: Integration, Stokes', Faraday's law', J. Japan Soc. Appl. Electromagnetics Mech. 6, 233-240.

A. Bossavit (1998e), 'On the geometry of electromagnetism IV: "Maxwell's house", J. Japan Soc. Appl. Electromagnetics Mech. 6, 318-326.

A. Bossavit (2001), Generalized finite differences in computational electromagnetics, in Geometric Methods for Computational Electromagnetics (F. Teixeira, ed.), Vol. 32 of PIER, PIERS, Boston, MA, pp. 45-64.

A. Bossavit and L. Kettunen (1999a), 'Yee-like schemes on a tetrahedral mesh with diagonal lumping', Internat. J. Numer. Modelling 12, 129-142.

A. Bossavit and L. Kettunen (1999b), Yee-like schemes on staggered cellular grids: A synthesis between FIT and FEM approaches. Contribution to COMPUMAG '99.

R. Bott and L. Tu (1982), Differential Forms in Algebraic Topology, Springer, New York.

D. Braess and C. Schwab (2000), 'Approximation on simplices with respect to weighted Sobolev norms', J. Approx. Theory 103, 329-337.

F. Brezzi and M. Fortin (1991), Mixed and Hybrid Finite Element Methods, Springer.

F. Brezzi, J. Douglas and D. Marini (1985), 'Two families of mixed finite elements for 2nd order elliptic problems', Numer. Math. 47, 217-235.

F. Brezzi, J. Rappaz and P. Raviart (1980), 'Finite dimensional approximation of nonlinear problems, Part I: Branches of nonsingular solutions', Numer. Math. 36, $1-25$.

A. Buffa, Y. Mayday and F. Rapetti (2001), 'A sliding mesh mortar method for two dimensional eddy currents model for electric engines', RAIRO M2AN 35, 191-228. 
A. Buffa, R. Hiptmair, T. von Petersdorff and C. Schwab (2002), 'Boundary element methods for Maxwell equations on Lipschitz domains'. To appear in Numer. Math.

W. Burke (1985), Applied Differential Geometry, Cambridge University Press, Cambridge.

S. Caorsi, P. Fernandes and M. Raffetto (1999), Approximations of electromagnetic eigenproblems: A general proof of convergence for edge finite elements of any order of both Nédélec's families. Technical Report 16/99, CNR-IMA Genoa, Genoa, Italy.

S. Caorsi, P. Fernandes and M. Raffetto (2000), 'On the convergence of Galerkin finite element approximations of electromagnetic eigenproblems', SIAM J. Numer. Anal. 38, 580-607.

H. Cartan (1967), Formes Différentielles, Hermann, Paris.

S. Christiansen (2000), Discrete Fredholm properties and convergence estimates for the EFIE. Technical Report 453, CMAP, Ecole Polytechique, Paris, France.

P. Ciarlet, Jr, and J. Zou (1999), 'Fully discrete finite element approaches for timedependent Maxwell equations', Numer. Math. 82, 193-219.

P. G. Ciarlet (1978), The Finite Element Method for Elliptic Problems, Vol. 4 of Studies in Mathematics and its Applications, North-Holland, Amsterdam.

M. Clemens and T. Weiland (1999), 'Transient eddy current calculation with the FI-method', IEEE Trans. Magnetics 35, 1163-1166.

M. Clemens and T. Weiland (2001), Discrete electromagnetism with the finite integration technique, in Geometric Methods for Computational Electromagnetics (F. Teixeira, ed.), Vol. 32 of PIER, PIERS, Boston, MA, pp. 65-87.

D. Colton and R. Kress (1998), Inverse Acoustic and Electromagnetic Scattering Theory, Vol. 93 of Applied Mathematical Sciences, 2nd edn, Springer, Heidelberg.

M. Costabel (1990), 'A remark on the regularity of solutions of Maxwell's equations on Lipschitz domains', Math. Meth. Appl. Sci. 12, 365-368.

M. Costabel (1991), 'A coercive bilinear form for Maxwell's equations', J. Math. Anal. Appl. 157, 527-541.

M. Costabel and M. Dauge (1998), Singularities of Maxwell's equations on polyhedral domains, in Analysis, Numerics and Applications of Differential and Integral Equations (M. Bach, ed.), Vol. 379 of Longman Pitman Res. Notes Math. Ser., Addison Wesley, Harlow, pp. 69-76.

M. Costabel and M. Dauge (1999), 'Maxwell and Lamé eigenvalues on polyhedra', Math. Methods Appl. Sci. 22, 243-258.

M. Costabel and M. Dauge (2002), 'Weighted regularization of Maxwell equations in polyhedral domains'. To appear in Numer. Math.

M. Costabel, M. Dauge and S. Nicaise (1999), 'Singularities of Maxwell interface problems', RAIRO M2AN 33, 627-649.

M. Dauge (1988), Elliptic Boundary Value Problems on Corner Domains, Vol. 1341 of Lecture Notes in Mathematics, Springer, Berlin.

R. Dautray and J.-L. Lions (1990), Mathematical Analysis and Numerical Methods for Science and Technology, Vol. 4, Springer, Berlin. 
L. Demkowicz (2001), Edge finite elements of variable order for Maxwell's equations: A discussion, in Scientific Computing in Electrical Engineering: Proceedings of a workshop held at Rostock, Germany, Aug 20-23 2000 (U. van Rienen, M. Günther and D. Hecht, eds), Vol. 18 of Lecture Notes in Computer Science and Engineering, Springer, Berlin, pp. 15-34.

L. Demkowicz and I. Babuška (2001), Optimal $p$ interpolation error estimates for edge finite elements of variable order in 2D. Technical Report 01-11, TICAM, University of Texas, Austin, TX.

L. Demkowicz, P. Monk, C. Schwab and L. Vardapetyan (1999), Maxwell eigenvalues and discrete compactness in two dimensions. Report 99-12, TICAM, University of Texas, Austin, TX.

G. Deschamps (1981), 'Electromagnetics and differential forms', Proc. IEEE 69, 676-695.

A. Dezin (1995), Multidimensional Analysis and Discrete Models, CRC Press, Boca Raton, FL, USA.

J. Dodziuk (1976), 'Finite-difference approach to the Hodge theory of harmonic forms', Amer. J. Math. 98, 79-104.

T. Dupont and R. Scott (1980), 'Polynomial approximation of functions in Sobolev spaces', Math. Comp. 34, 441-463.

H. Federer (1969), Geometric Measure Theory, Springer, New York.

P. Fernandes and G. Gilardi (1997), 'Magnetostatic and electrostatic problems in inhomogeneous anisotropic media with irregular boundary and mixed boundary conditions', Math. Models Meth. Appl. Sci., M3AS 7, 957-991.

P. Fernandes and M. Raffetto (2000), Recent developments in the (spurious-free) approximation of electromagnetic eigenproblems by the finite element method. Technical Report 06/00, CNR-IMA Genoa, Genoa, Italy.

R. Fritsch and R. Piccini (1990), Cellular Structures in Topology, Vol. 19 of Cambridge Studies in Advanced Mathematics, Cambridge University Press.

V. Girault and P. Raviart (1986), Finite Element Methods for Navier-Stokes Equations, Springer, Berlin.

J. Gopalakrishnan and J. Pasciak (2000), Overlapping Schwarz preconditioners for indefinite time harmonic Maxwell equations. Technical report, Department of Mathematics, Texas A\&M University. Submitted to Math. Comp.

V. Gradinaru and R. Hiptmair (1999), 'Whitney elements on pyramids', Electron. Trans. Numer. Anal. 8, 154-168.

H. Grauert and I. Lieb (1977), Differential- und Integralrechnung III, 3rd edn, Springer, Berlin.

P. Grisvard (1985), Elliptic Problems in Nonsmooth Domains, Pitman, Boston.

P. Gross and P. Kotiuga (2001), Data structures for geometric and topological aspects of finite element algorithms, in Geometric Methods for Computational Electromagnetics (F. Teixeira, ed.), Vol. 32 of PIER, PIERS, Boston, MA, pp. 151-169.

M. Grote and J. Keller (1998), 'Nonreflecting boundary conditions for Maxwell's equations', J. Comput. Phys. 139, 327-342. 
E. Haber, U. Ascher, D. Aruliah and D. Oldenburg (1999), Fast modelling of 3D electromagnetic problems using potentials. Technical report, Department of Computer Science, University of British Columbia, Vancouver, Canada. Submitted to J. Comput. Phys.

W. Hackbusch (1993), Iterative Solution of Large Sparse Systems of Equations, Vol. 95 of Applied Mathematical Sciences, Springer, New York.

M. Hanna and K. Smith (1967), 'Some remarks on the Dirichlet problem in piecewise smooth domains', Comm. Pure Appl. Math. 20, 575-593.

C. Hazard and M. Lenoir (1996), 'On the solution of time-harmonic scattering problems for Maxwell's equations', SIAM J. Math. Anal. 27, 1597-1630.

C. Hazard and S. Lohrengel (2000), A singular field method for Maxwell's equations: Numerical aspects in two dimensions. Submitted.

R. Hiptmair (1999a), 'Canonical construction of finite elements', Math. Comp. 68, 1325-1346.

R. Hiptmair (1999b), 'Multigrid method for Maxwell's equations', SIAM J. Numer. Anal. 36, 204-225.

R. Hiptmair (2000), Symmetric coupling for eddy current problems. Technical Report 148, Sonderforschungsbereich 382, Universität Tübingen, Tübingen, Germany. To appear in SIAM J. Numer. Anal.

R. Hiptmair (2001a), 'Discrete Hodge operators'. To appear in Numer. Math. Published online May 30, 2001, http://dx.doi.org/10.1007/s002110100295.

R. Hiptmair (2001b), 'Discrete Hodge operators: An algebraic perspective', in Geometric Methods for Computational Electromagnetics (F. Teixeira, ed.), Vol. 32 of PIER, EMW Publishing, Cambridge, MA, pp. 247-269.

R. Hiptmair (2001c), 'Higher order Whitney forms', in Geometric Methods for Computational Electromagnetics (F. Teixeira, ed.), Vol. 32 of PIER, EMW Publishing, Cambridge, MA, pp. 271-299.

R. Hiptmair and A. Toselli (1999), Overlapping and multilevel Schwarz methods for vector valued elliptic problems in three dimensions, in Parallel Solution of Partial Differential Equations (P. Bjorstad and M. Luskin, eds), Vol 120 of IMA Volumes in Mathematics and its Applications, Springer, Berlin, pp. 181202.

R. Hoppe (1999), 'Mortar edge element methods in $\mathbb{R}^{3}$, East-West J. Numer. Anal. $7,159-175$.

J. Hyman and M. Shashkov (1999), 'Mimetic discretizations for Maxwell's equations', J. Comput. Phys. 151, 881-909.

J. Hyman and M. Shashkov (2001), Mimetic finite difference methods for Maxwell's equations and the equations of magnetic diffusion, in Geometric Methods for Computational Electromagnetics (F. Teixeira, ed.), Vol. 32 of PIER, PIERS, Boston, MA, pp. 89-121.

F. Ihlenburg (1998), Finite Element Analysis of Acoustic Scattering, Vol. 132 of Applied Mathematical Sciences, Springer, New York.

T. Iwaniec (1999), Nonlinear differential forms, in Lecture Notes of the International Summer School in Jyväskylä, 1998, Department of Mathematics, University of Jyväskylä, Finland.

F. Jochmann (1997), 'A compactness result for vector fields with divergence and curl in $L^{q}(\Omega)$ involving mixed boundary conditions', Appl. Anal. 66, 189-203. 
B. Khoromskij and J. Melenk (2001), Boundary concentrated finite element methods. Preprint 45, MPI Leipzig, Germany.

F. Kikuch (1987), 'Mixed and penalty formulations for finite element analysis of an eigenvalue problem in electromagnetism', Comput. Methods Appl. Mech. Engr. 64, 509-521.

F. Kikuchi (1989), 'On a discrete compactness property for the Nédélec finite elements', J. Fac. Sci., Univ. Tokyo, Sect. I A 36, 479-490.

A. Kirsch and P. Monk (2000), 'A finite element method for approximating electromagnetic scattering from a conducting object'. To appear in Numer. Math.

V. Kondrat'ev (1967), 'Boundary value problems for elliptic equations in domains with conical or angular points', Trans. Moscow Math. Soc. 16, 227-313.

R. Kress (1971), 'Ein kombiniertes Dirichlet-Neumannsches Randwertproblem bei harmonischen Vektorfeldern', Arch. Rational Mech. Anal. 42, 40-49.

R. Kress (1989), Linear Integral Equations, Vol. 82 of Applied Mathematical Sciences, Springer, Berlin.

M. Kuhn and O. Steinbach (2001), 'FEM-BEM coupling for 3d exterior magnetic field problems'. To appear in Math. Meth. Appl. Sci.

S. Lang (1995), Differential and Riemannian Manifolds, Vol. 160 of Graduate Texts in Mathematics, Springer, New York.

R. Leis (1986), Initial Boundary Value Problems in Mathematical Physics, B.G. Teubner, Stuttgart.

S. Lohrengel and S. Nicaise (2001), 'Singularities and density problems for composite materials in electromagnetism'. Submitted to Comm. Part. Diff. Equ.

A. Lundell and S. Weingram (1969), The Topology of CW Complexes, The University Series in Higher Mathematics, Van Nostrand Reinhold Company, New York.

M. Marrone (2001), Computational aspects of the cell method in electrodynamics, in Geometric Methods for Computational Electromagnetics (F. Teixeira, ed.), Vol. 32 of PIER, PIERS, Boston, MA, pp. 317-356.

C. Mattiussi (2000), 'The finite volume, finite element and finite difference methods as numerical methods for physical field problems', Adv. Imaging Electron Physics 113, 1-146.

J. Maxwell (1891), A Treatise on Electricity and Magnetism, Oxford University Press reprint, 3rd edn (1998).

V. Maz'ya (1985), Sobolev Spaces, Springer, Berlin.

C. Mercat (2001), 'Discrete Riemann surfaces and the Ising model', Commun. Math. Phys. 218, 177-216.

P. Monk (1992a), 'Analysis of a finite element method for Maxwell's equations', SIAM J. Numer. Anal. 29, 714-729.

P. Monk (1992b), 'A finite element method for approximating the time-harmonic Maxwell equations', Numer. Math. 63, 243-261.

P. Monk (1994), 'On the $p$ and $h p$-extension of Nédélec's conforming elements', J. Comput. Appl. Math. 53, 117-137.

P. Monk (1999), 'A posteriori error indicators for Maxwell's equations'. To appear in J. Comput. Appl. Math.

P. Monk (2001), 'A simple proof of convergence for an edge element discretization of Maxwell's equations'. Submitted to Math. Comp. 
P. Monk and L. Demkowicz (2001), 'Discrete compactness and the approximation of Maxwell's equations in $\mathbb{R}^{3}$, Math. Comp. 70, 507-523.

F. Morgan (1995), Geometric Measure Theory: A Beginner's Guide, 2nd edn, Academic Press, San Diego, CA.

W. Müller (1978), 'Analytic torsion and R-torsion of Riemannian manifolds', Adv. Math. 28, 233-305.

R. Munoz-Solar (1997), 'Polynomial liftings on a tetrahedron and applications to the $h p$-version of the finite element method in three dimensions', SIAM J. Numer. Anal. 34, 282-314.

S. Nazarov and B. Plamenevskii (1994), Elliptic Problems in Domains with Piecewise Smooth Boundaries, Vol. 13 of Expositions in Mathematics, Walter de Gruyter, Berlin.

J. Nédélec (1980), 'Mixed finite elements in $R^{3}$ ', Numer. Math. 35, 315-341.

J. Nédélec (1986), 'A new family of mixed finite elements in $R^{3}$ ', Numer. Math. 50, $57-81$.

J.-C. Nédélec (2001), Acoustic and Electromagnetic Equations: Integral Representations for Harmonic Problems, Vol. 44 of Applied Mathematical Sciences, Springer, Berlin.

S. Nicaise (2001), 'Edge elements on anisotropic meshes and approximation of the Maxwell equations', SIAM J. Numer. Anal. 39, 784-816.

R. Nicolaides and D.-Q. Wang (1998), 'Convergence analysis of a covolume scheme for Maxwell's equations in three dimensions', Math. Comp. 67, 947-963.

R. Nicolaides and X. Wu (1997), 'Covolume solutions of three-dimensional div-curl equations', SIAM J. Numer. Anal. 34, 2195-2203.

J. Osborn (1975), 'Spectral approximation for compact operators', Math. Comp. 29, 712-725.

L. Pavarino and O. Widlund (1996), 'A polylogarithmic bound for an iterative substructuring method for spectral elements in three dimensions', SIAM J. Numer. Anal. 33, 1303-1335.

R. Picard (1984), 'An elementary proof for a compact imbedding result in generalized electromagnetic theory', Math. Z. 187, 151-161.

W. Rachowicz and L. Demkowicz (2002), 'An $h p$-adaptive finite element method for electromagnetics, Part II: A 3D implementation', Internat. J. Numer. Meth. Engr. 53, 147-180.

P. A. Raviart and J. M. Thomas (1977), A Mixed Finite Element Method for Second Order Elliptic Problems, Vol. 606 of Springer Lecture Notes in Mathematics, Springer, New York, pp. 292-315.

Z. Sacks, D. Kingsland, R. Lee and J.-F. Lee (1995), 'A perfectly matched anisotropic absorber for use as an absorbing boundary condition', IEEE Trans. Antennas Propag. 43, 1460-1463.

A. Schatz and J. Wang (1996), 'Some new error estimates for Ritz-Galerkin methods with minimal regularity assumptions', Math. Comp. 65, 19-27.

R. Schuhmann and T. Weiland (2001), Conservation of discrete energy and related laws in the finite integration technique, in Geometric Methods for Computational Electromagnetics (F. Teixeira, ed.), Vol. 32 of PIER, PIERS, Boston, MA, pp. 301-316. 
C. Schwab (1998), p- and hp-Finite Element Methods: Theory and Applications in Solid and Fluid Mechanics, Numerical Mathematics and Scientific Computation, Clarendon Press, Oxford.

G. Schwarz (1995), Hodge Decomposition: A method for Solving Boundary Value Problems, Vol. 1607 of Springer Lecture Notes in Mathematics, Springer, Berlin.

D.-K. Sun, J.-F. Lee and Z. Cendes (2001), 'Construction of nearly orthogonal Nédélec bases for rapid convergence with multilevel preconditioned solvers', SIAM J. Sci. Comput. 23, 1053-1076.

A. Taflove (1995), The Finite Difference in Time Domain Method, Artech House, Boston, London.

T. Tarhasaari and L. Kettunen (2001), Topological approach to computational electromagnetism, in Geometric Methods for Computational Electromagnetics (F. Teixeira, ed.), Vol. 32 of PIER, PIERS, Boston, MA, pp. 189-206.

T. Tarhasaari, L. Kettunen and A. Bossavit (1999), 'Some realizations of a discrete Hodge: A reinterpretation of finite element techniques', IEEE Trans. Magnetics 35, 1494-1497.

F. Teixeira (2001), Geometric aspects of the simplicial discretization of Maxwell's equations, in Geometric Methods for Computational Electromagnetics (F. Teixeira, ed.), Vol. 32 of PIER, PIERS, Boston, MA, pp. 171-188.

F. Teixeira and C. Chew (1998), 'A general approach to extend Berenger's absorbing boundary conditions to anisotropic and dispersive media', IEEE Trans. Antennas Propag. 46, 1386-1387.

F. Teixeira and W. Chew (1999), 'Lattice electromagnetic theory from a topological viewpoint', J. Math. Phys. 40, 169-187.

E. Tonti (1996), On the geometrical structure of electromagnetism, in Gravitation, Electromagnetism and Geometrical Structures (G. Ferrarese, ed.), Pitagora, Bologna, Italy, pp. 281-308.

E. Tonti (2001), Finite formulation of the electromagnetic field, in Geometric Methods for Computational Electromagnetics (F. Teixeira, ed.), Vol. 32 of PIER, PIERS, Boston, MA, pp. 1-44.

L. Vardapetyan and L. Demkowicz (1999), ' $h p$-adaptive finite elements in electromagnetics', Comput. Meth. Appl. Mech. Engr. 169, 331-344.

C. Weber (1980), 'A local compactness theorem for Maxwell's equations', Math. Meth. Appl. Sci. 2, 12-25.

T. Weiland (1996), 'Time domain electromagnetic field computation with finite difference methods', Internat. J. Numer. Modelling 9, 295-319.

P. Werner (1963), 'On the exterior boundary value problem of perfect reflexion for stationary electromagnetic wave fields', J. Math. Anal. Appl. 7, 348-396.

H. Whitney (1957), Geometric Integration Theory, Princeton University Press, Princeton.

K. Yee (1966), 'Numerical solution of initial boundary value problems involving Maxwell's equations in isotropic media', IEEE Trans. Antennas Propag. 16, 302-307. 


\section{Appendix: Symbols and notation}

\begin{tabular}{|c|c|}
\hline$A\left(\mathbb{R}^{3}\right)$ & Three-dimensional affine space \\
\hline e & Complex amplitude of electric field \\
\hline b & Complex amplitude of magnetic induction \\
\hline d & Complex amplitude of displacement current \\
\hline $\mathbf{h}$ & Complex amplitude of magnetic field \\
\hline$\epsilon$ & Dielectric tensor \\
\hline $\boldsymbol{\mu}$ & Tensorial magnetic permeability \\
\hline$\sigma$ & Conductivity tensor \\
\hline $\mathcal{M}$ & (Piecewise smooth) manifold \\
\hline $\mathcal{S}_{l}$ & $\begin{array}{l}\text { Oriented, piecewise smooth sub-manifolds of } \mathcal{M} \\
\text { (Definition 1) }\end{array}$ \\
\hline $\mathcal{F}^{l}(\mathcal{M})$ & $\begin{array}{l}\text { Space of integral } l \text {-forms on manifold } \mathcal{M}, l \in \mathbb{N} \\
\text { (Definition 1) }\end{array}$ \\
\hline$\times$ & Cross product of vectors in $\mathbb{R}^{3}$ \\
\hline $\mathcal{D} \mathcal{F}^{l, m}(\mathcal{M})$ & $\begin{array}{l}\text { Differential forms of class } C^{m} \text { on smooth manifold } \mathcal{M} \\
\text { (Definition 2) }\end{array}$ \\
\hline$F_{\mathcal{M}}^{l}$ & Injection of differential $l$-forms into integral $l$-forms \\
\hline$\Upsilon_{l}$ & $\begin{array}{l}\text { Association of (piecewise) continuous } l \text {-forms and vector } \\
\text { proxies in three-dimensional Euclidean space (Table } 2.1 \text { ) }\end{array}$ \\
\hline $\mathbf{n}$ & $\begin{array}{l}\text { (Exterior) unit normal vector-field on some oriented } \\
\text { two-dimensional Lipschitz surface }\end{array}$ \\
\hline$d$ & Exterior derivative for (differential/integral) $l$-forms \\
\hline$\Phi^{*}$ & $\begin{array}{l}\text { Pullback operator belonging to diffeomorphism } \boldsymbol{\Phi} \\
\text { (see }(2.12) \text { ) }\end{array}$ \\
\hline $\mathbf{t}_{\mathcal{N}}$ & $\begin{array}{l}\text { Trace of integral/differential forms onto a sub-manifold } \\
\text { (see }(2.14) \text { ) }\end{array}$ \\
\hline $\mathfrak{F}_{\mathcal{M}}^{l}$ & $\begin{array}{l}\text { Pullbacks for vector proxies of } l \text {-forms in } \\
\text { three-dimensional Euclidean space (see }(2.16)-(2.19))\end{array}$ \\
\hline$\gamma$ & Standard trace operator, i.e., pointwise restriction \\
\hline$\gamma_{\mathbf{t}}$ & Tangential trace $\mathbf{u} \mapsto \mathbf{u} \times \mathbf{n}_{\mid \Gamma}$ \\
\hline$\gamma_{\mathbf{n}}$ & Normal trace $\mathbf{u} \mapsto \mathbf{u} \cdot \mathbf{n}_{\mid \Gamma}$ \\
\hline$\wedge$ & Exterior product of differential forms \\
\hline$\Omega$ & (Curvilinear) Lipschitz polyhedron $\subset A\left(\mathbb{R}^{3}\right)$ \\
\hline$\Gamma$ & Oriented boundary of $\Omega$ \\
\hline
\end{tabular}




\begin{tabular}{|c|c|}
\hline $\boldsymbol{H}(\operatorname{curl} ; \Omega)$ & $:=\left\{\mathbf{v} \in \boldsymbol{L}^{2}(\Omega)\right.$, curl $\left.\mathbf{v} \in \boldsymbol{L}^{2}(\Omega)\right\}$ \\
\hline $\boldsymbol{H}(\operatorname{div} ; \Omega)$ & $:=\left\{\mathbf{v} \in \boldsymbol{L}^{2}(\Omega), \operatorname{div} \mathbf{v} \in \boldsymbol{L}^{2}(\Omega)\right\}$ \\
\hline $\boldsymbol{H}_{0}(\operatorname{curl} ; \Omega)$ & $:=\left\{\mathbf{v} \in \boldsymbol{H}(\operatorname{curl} ; \Omega), \gamma_{\mathbf{t}} \mathbf{v}=0\right\}$ \\
\hline $\boldsymbol{H}_{0}(\operatorname{div} ; \Omega)$ & $:=\left\{\mathbf{v} \in \boldsymbol{H}(\operatorname{div} ; \Omega), \gamma_{\mathbf{n}} \mathbf{v}=0\right\}$ \\
\hline $\boldsymbol{H}(\operatorname{curl} 0 ; \Omega)$ & $:=\{\mathbf{v} \in \boldsymbol{H}(\operatorname{curl} ; \Omega), \operatorname{curl} \mathbf{v}=0\}$ \\
\hline $\boldsymbol{H}(\operatorname{div} 0 ; \Omega)$ & $:=\{\mathbf{v} \in \boldsymbol{H}(\operatorname{div} ; \Omega), \operatorname{div} \mathbf{v}=0\}$ \\
\hline $\mathcal{H}^{1}(\Omega)$ & $\begin{array}{l}\text { Space of harmonic Neumann vector-fields, contained in } \\
\boldsymbol{H}(\boldsymbol{c u r l} 0 ; \Omega) \cap \boldsymbol{H}(\operatorname{div} 0 ; \Omega) \text { (see Lemma } 2.2)\end{array}$ \\
\hline $\mathcal{H}^{2}(\Omega)$ & $\begin{array}{l}\text { Space of harmonic Dirichlet vector-fields, contained in } \\
\boldsymbol{H}(\operatorname{curl} 0 ; \Omega) \cap \boldsymbol{H}(\operatorname{div} 0 ; \Omega)\end{array}$ \\
\hline$\Omega_{h}$ & $\begin{array}{l}\text { Triangulation/mesh (see Definition } 3 \text { ) of } \Omega \text { with } \\
\text { meshwidth } h \text { according to }(3.34)\end{array}$ \\
\hline $\mathcal{S}_{l}\left(\Omega_{h}\right)$ & Set of $l$-facets of triangulation $\Omega_{h}$ \\
\hline $\mathcal{C}^{l}\left(\Omega_{h}\right)$ & Vector space of $l$-cochains on the mesh $\Omega_{h}$ \\
\hline $\mathrm{D}^{l}$ & Matrix of exterior derivative on $l$-cochains (see Section 3.1) \\
\hline $\mathrm{I}_{l}$ & deRham maps $\mathcal{F}^{l}(\Omega) \mapsto \mathcal{C}^{l}\left(\Omega_{h}\right)($ see $(3.2))$ \\
\hline $\mathrm{W}^{l}$ & Interpolation of $l$-cochains (Whitney map, see Section 3.2) \\
\hline $\mathcal{W}_{p}^{l}\left(\Omega_{h}\right)$ & $\begin{array}{l}\text { Space of Whitney } l \text {-forms of uniform polynomial degree } p \\
\text { on mesh } \Omega_{h} \text { (see Definition } 5 \text { for } p=0 \text {, and Section } 3.4 \\
\left.\text { for general } p \in \mathbb{N}_{0}\right)\end{array}$ \\
\hline $\mathcal{W}_{p, 0}^{l}\left(\Omega_{h}\right)$ & Discrete differential $l$-forms with vanishing trace on $\partial \Omega$ \\
\hline$\Pi^{l}$ & $\begin{array}{l}\text { Local interpolation operators for Whitney } l \text {-forms } \\
\text { (see }(3.11))\end{array}$ \\
\hline $\mathcal{P}_{p}(T)$ & Space of multivariate polynomials of total degree $\leq p$ on $T$ \\
\hline$\widetilde{\mathcal{P}}_{p}(T)$ & $\begin{array}{l}\text { Space of multivariate homogeneous polynomials on } T \text { of } \\
\text { exact total degree } p\end{array}$ \\
\hline $\mathcal{D} \mathcal{P}_{p}^{l}(T)$ & $\begin{array}{l}\text { Polynomial differential } l \text {-forms of polynomial degree } \leq p \\
\text { on simplex } T \text { (see }(3.15))\end{array}$ \\
\hline $\mathcal{X}_{p}^{l}(S)$ & $\begin{array}{l}\text { Local space of } p \text {-order Whitney forms associated with } \\
\text { facet } S\end{array}$ \\
\hline $\mathcal{Z}_{p}^{l}(S)$ & $\begin{array}{l}\text { Polynomial } l \text {-forms of degree } \leq p \text { and vanishing trace on } \\
\partial S \text { (see }(3.18))\end{array}$ \\
\hline $\mathrm{E}_{p, S}^{l}$ & $\begin{array}{l}\text { Barycentric extension of a polynomial } l \text {-form from a facet } \\
\text { into a cell (see Section 3.4) }\end{array}$ \\
\hline $\mathfrak{K}^{l}$ & Poincaré mapping $\mathcal{D} \mathcal{F}^{l, 0}(T) \mapsto \mathcal{D} \mathcal{F}^{l-1,1}(T)$ (see (3.21)) \\
\hline
\end{tabular}


$\check{\mathcal{W}}_{p}^{l}\left(\Omega_{h}\right) \quad$ Second family of Whitney $l$-forms of polynomial degree $p$ on mesh $\Omega_{h}$ (see (3.28))

$\underline{\mathbf{p}} \quad$ Polynomial degree vector in the variable degree $p$-version of discrete differential forms (see Section 3.4)

$\Theta_{\mathbf{p}}^{l}\left(\Omega_{h}\right) \quad$ Set of degrees of freedom for $\mathcal{W}_{\mathbf{p}}^{l}\left(\Omega_{h}\right)$ (see Section 3.5)

$\mathrm{PL}_{S} \quad$ Potential liftings of closed polynomial forms on simplex $S$

$\mathrm{PE}_{S}^{l} \quad$ Polynomial extension operators respecting vanishing traces

$\mathrm{PQ}_{S}^{l} \quad$ Projections onto closed polynomial forms

I $3 \times 3$ identity matrix

$\boldsymbol{X}_{0}(\boldsymbol{\alpha}, \Omega) \quad:=\left\{\mathbf{u} \in \boldsymbol{H}_{0}(\mathbf{c u r l} ; \Omega), \operatorname{div}(\boldsymbol{\alpha} \mathbf{u}) \in L^{2}(\Omega)\right\}$ (see Section 4.1)

$\boldsymbol{Z}_{0}(\boldsymbol{\alpha}, \Omega) \quad \boldsymbol{\alpha}$-Orthogonal complement in $\boldsymbol{H}_{0}(\operatorname{curl} ; \Omega)$ of kernel of curl (see (4.3))

$\boldsymbol{Z}_{h, 0}\left(\boldsymbol{\alpha}, \Omega_{h}\right) \quad$ Discrete counterpart of $\boldsymbol{Z}_{0}(\boldsymbol{\alpha}, \Omega)$

$\mathrm{H}_{\boldsymbol{\epsilon}} \quad$ Hodge mapping $\boldsymbol{H}_{0}(\operatorname{curl} ; \Omega) \mapsto \boldsymbol{Z}_{0}(\boldsymbol{\epsilon}, \Omega)$ (see (4.8))

$\mathrm{F}_{h} \quad$ Fortin projector $\boldsymbol{H}_{0}(\operatorname{curl} ; \Omega) \mapsto \boldsymbol{Z}_{h, 0}\left(\boldsymbol{\epsilon}, \Omega_{h}\right)$ (see (4.10))

$\mathrm{Q}_{h}^{\boldsymbol{\alpha}} \quad \boldsymbol{\alpha}$-Orthogonal projection onto $\operatorname{curl} \mathcal{W}_{p, 0}^{1}\left(\Omega_{h}\right)$

$\mathrm{P}^{\perp} \quad$ Orthogonal projection onto $\boldsymbol{Z}_{0}(\mathbf{I}, \Omega)$

$\mathrm{P}^{0} \quad$ Orthogonal projection onto $\boldsymbol{H}_{0}(\operatorname{curl} 0 ; \Omega)$

$\mathcal{S}_{p, 0}\left(\Omega_{h}\right) \quad$ Space of piecewise polynomial continuous vector-fields on $\Omega_{h}$

$\boldsymbol{H}_{\times}^{1}(\Omega) \quad$ Space of vector-fields in $\boldsymbol{H}^{1}(\Omega)$ with vanishing tangential components on $\partial \Omega$ 